\title{
Bilaterale Koordination \\ kommissuraler Interneurone im Mesothorakalganglion von \\ Locusta migratoria migratorioides
}

\author{
Dissertation \\ zur Erlangung des Doktorgrades \\ der Mathematisch-Naturwissenschaftlichen Fakultäten \\ der Georg-August-Universität zu Göttingen
}

vorgelegt von

Marian Baldus

aus Fürstenfeldbruck

Göttingen 2008 
D 7

Referent:

Korreferent:

Tag der mündlichen Prüfung:
Prof. Dr. R. Hustert

Prof. Dr. R. Heinrich

31. 10. 2008 


\section{Inhaltsverzeichnis}

1 Einleitung 1

2 Versuchstiere, Material und Methoden 12

2.1 Auswahl der Versuchstiere 12

2.2 Konstruktion einer rotierbaren Laufvorrichtung für gehalterte Heuschrecken unter den Bedingungen der elektrophysiologischen intrazellulären Ableitung

2.3 Präparation

2.3.1 Chronische Muskelableitungen vom Depressor trochanteris (M103a) beider Mittelbeine

2.3.2 Präparation eines semi-intakten Präparates für intrazelluläre Ableitungen von Interneuronen des Mesothorakalganglions

2.4 Versuchsaufbau

2.5 Versuchsdurchführung 22

2.5.1 Intrazelluläre Ableitung, Reizung und Datenaufnahme 22

2.5.2 Intrazelluläre Färbungen mit Lucifer Yellow, histologische Aufarbeitung für die Fluoreszenz-Mikroskopie und zweidimensionale Rekonstruktion der neuronalen Morphologie durch Zeichnung

2.5.3 Auswertung der physiologischen Daten

2.6 Nomenklatur

2.6.1 Nomenklatur der beschrieben kommissuralen Interneurone

2.6.2 Nomenklatur bekannter morphologischer Strukturen im Mesothorakalganglion

2.6.3 Erläuterungen $\mathrm{zu}$ einigen bei der Beschreibung von Morphologie und Physiologie der Neurone verwendeten Begrifflichkeiten

2.7 Anfertigung medianer Sagittalschnitte vom Mesothorakalganglion, Aufbereitung und Analyse unter dem Transmissionselektronenmikroskop 
2.8.1 Ringer-Lösung nach CLEMENTS und MAY (1974) 36

2.8.2 Paraformaldehyd, 4\%ige Lösung 36

2.8.3 Phosphate buffered saline (PBS Puffer; $\mathrm{pH}=7,4$ ) 36

2.8.4 Fixierung und Einbettung von medianen Sagittalschnitten 37 (Mesothorakalganglion) für die Transmissionselektronenmikroskopie

2.9 Liste der verwendeten Abkürzungen

3.1 Detaillierte Beschreibung von morphologischen und physiologischen Merkmalen von ausgewählten Typ CIN-I- und Typ CIN-II Interneuronen

3.1.1 Bilateral verzweigtes kommissurales Typ CIN-I Interneuron $\mathrm{CIN}-\mathrm{I}(\mathrm{ad}) \mathrm{C} 1$

3.1.2 Bilateral verzweigtes kommissurales Typ CIN-I Interneuron $\mathrm{CIN}-\mathrm{I}(\mathrm{a}) \mathrm{c} 1$

3.1.3 Bilateral verzweigtes kommissurales Typ CIN-I Interneuron $\mathrm{CIN}-\mathrm{I}(\mathrm{d}) \mathrm{c} 1$

3.1.4 Bilateral verzweigtes kommissurales Typ CIN-I Interneuron $\mathrm{CIN}-\mathrm{I}(\mathrm{d}) \mathrm{c} 2$

3.1.5 Median verzweigtes kommissurales Typ CIN-II Interneuron $\mathrm{CIN}-\mathrm{II}(\mathrm{ad}) \mathrm{c} 1$

3.1.6 Median verzweigtes kommissurales Typ CIN-II Interneuron $\mathrm{CIN}-\mathrm{II}(\mathrm{d}) \mathrm{c} 1$

3.1.7 Median verzweigtes kommissurales Typ CIN-II Interneuron $\mathrm{CIN}-\mathrm{II}(\mathrm{a}) \mathrm{i1}$

3.2 Allgemeine morphologische und physiologische Merkmale von kommissuralen intersegmentalen Interneuronen

3.2.1 Kommissurale Interneurone vom Typ CIN-I mit bilateralem Verzweigungsmuster

3.2.2 Kommissurale Interneurone vom Typ CIN-II mit medianem Verzweigungsmuster

3.3 Licht- und elektronenmikroskopische Aufnahmen von medianen 
4 Diskussion

4.1 Allgemeine Erkenntnisse über kommissurale Interneurone im Mesothorakalganglion von Wanderheuschrecken unabhängig von ihrem Verzweigungstyp

4.2 Welche Rolle spielen intersegmentale kommissurale Interneurone vom Typ CIN-I bei der bilateralen (intrasegmentalen) Koordination der Extremitäten?

4.3 Welche Rolle spielen intersegmentale kommissurale Interneurone vom Typ CIN-II bei der bilateralen (intrasegmentalen) Koordination der Extremitäten?

4.4 Welche Rolle spielen lokale kommissurale Interneurone im Kontext der bilateralen Koordination der Extremitäten?

4.5 Vergleich der kommissuralen Interneurone vom Typ CIN-I und CIN-II 149 mit verschiedenen Klassen kommissuraler Interneurone, die für andere Tiergruppen beschrieben wurden

5 Zusammenfassung

6 Literaturverzeichnis 


\section{Abbildungsverzeichnis}

Abb. 1 Konstruktion für eine leicht rotierbare Laufkugel aus Styropor 13

Abb. 2 Implantierung der Myogrammelektroden in den Depressor trochan- 16 teris M103a

Abb. 3 Versuchsanordnung zur intrazellulären Ableitung von Inter- 21 neuronen des Mesothorakalganglions bei Locusta migratoria

Abb. 4 Morphologische Strukturen im Mesothorakalganglion von Locusta 30 migratoria, Übersicht 1

Abb. 5 Morphologische Strukturen im Mesothorakalganglion von Locusta migratoria, Übersicht 2

Abb. 6 Morphologie des kommissuralen Interneurons CIN-I(ad)c1;

bilateral verzweigter Typ I; (Zeichnung, MsG)

Abb. 7 Morphologie des kommissuralen Interneurons CIN-I(ad)c1;

bilateral verzweigter Typ I; (Fotografie, MsG)

Abb. 8 Physiologie des kommissuralen Interneurons CIN-I(ad)c1;

(kontralaterale Reizung)

Abb. 9 Physiologie des kommissuralen Interneurons CIN-I(ad)c1; (ipsilaterale Reizung)

Abb. 10 Physiologie des kommissuralen Interneurons CIN-I(ad)c1; (aktive Bewegung)

Abb. 11 Morphologie des kommissuralen Interneurons CIN-I(a)c1;

bilateral verzweigter Typ I; (Zeichnung, MsG)

Abb. 12 Morphologie des kommissuralen Interneurons CIN-I(a)c1;

bilateral verzweigter Typ I; (Fotografie, MsG)

Abb. 13 Physiologie des kommissuralen Interneurons CIN-I(a)c1; (kontralaterale Reizung, aktive Bewegung)

Abb. 14 Morphologie des kommissuralen Interneurons CIN-I(d)c1; bilateral verzweigter Typ I; (Zeichnung, MsG)

Abb. 15 Morphologie des kommissuralen Interneurons CIN-I(d)c1; 
Abb. 16 Physiologie des kommissuralen Interneurons CIN-I(d)c1;

(kontra- und ipsilaterale Reizung)

Abb. 17 Physiologie des kommissuralen Interneurons CIN-I(d)c1; (aktive Bewegung)

Abb. 18 Morphologie des kommissuralen Interneurons CIN-I(d)c2; bilateral verzweigter Typ I; (Zeichnung, MsG)

Abb. 19 Morphologie des kommissuralen Interneurons CIN-I(d)c2;

bilateral verzweigter Typ I; (Fotografie MsG, MtG; Zeichnung MtG)

Abb. 20 Physiologie des kommissuralen Interneurons CIN-I(d)c2; (ipsilaterale Reizung)

Abb. 21 Physiologie des kommissuralen Interneurons CIN-I(d)c2; (kontraterale Reizung, aktive Bewegung)

Abb. 22 Morphologie des kommissuralen Interneurons CIN-II(ad)c1; median verzweigter Typ II; (Zeichnung, MsG)

Abb. 23 Morphologie des kommissuralen Interneurons CIN-II(ad)c1; median verzweigter Typ II; (Fotografie MsG, MtG; Zeichnung MtG)

Abb. 24 Physiologie des kommissuralen Interneurons CIN-II(ad)c1; (ipsi- und kontralaterale Reizung)

Abb. 25 Morphologie des kommissuralen Interneurons CIN-II(d)c1; median verzweigter Typ II; (Zeichnung, MsG)

Abb. 26 Morphologie des kommissuralen Interneurons CIN-II(d)c1; median verzweigter Typ II; (Fotografie MsG, MtG, Zeichnung MtG)

Abb. 27 Physiologie des kommissuralen Interneurons CIN-II(d)c1; (kontralaterale Reizung)

Abb. 28 Physiologie des kommissuralen Interneurons CIN-II(d)c1; (aktive Bewegung)

Abb. 29 Morphologie des kommissuralen Interneurons CIN-II(a)i1; median verzweigter Typ II; (Zeichnung, MsG)

Abb. 30 Morphologie des kommissuralen Interneurons CIN-II(a)i1; median verzweigter Typ II, (Fotografie, MsG) 
Abb. 31 Physiologie des kommissuralen Interneurons CIN-II(a)i1; (kontralaterale Reizung)

Abb. 32 Physiologie des kommissuralen Interneurons CIN-II(a)i1; (ipsilaterale Reizung)

Abb. 33 Kommissurale Interneurone vom Typ I, Übersicht 1;

CIN-I(ad)c1, CIN-I(a)c1, CIN-I(d)c1, CIN-I(d)c2

Abb. 34 Kommissurale Interneurone vom Typ II, Übersicht 2; CIN-II(ad)c1, CIN-II(ad)c2, CIN-II(ad)c3, CIN-II(ad)c4

Abb. 35 Kommissurale Interneurone vom Typ II, Übersicht 3;

CIN-II(ad)c5, CIN-II(ad)c6, CIN-II(a)i1, CIN-II(a)i2

Abb. 36 Kommissurale Interneurone vom Typ II, Übersicht 4; CIN-II(a)c1, CIN-II(a)c2, CIN-II(d)c1, CIN-II(d)c2

Abb. 37 Dorsale Kommissuren im Mesothorakalganglion der Wanderheuschrecke; Licht- und elektronenmikroskopische Analyse von medianen Sagittalschnitten 
1 Einleitung

Bei vielen Interaktionen organismischer Körper mit der Umwelt in den verschiedensten Verhaltenskontexten ist eine kontinuierliche Aufrechterhaltung des Gleichgewichtes unabdingbar. Das betrifft in besonderem Maße auch fast alle Formen von Lokomotion, sei es nun solche, die durch schlängelnde Bewegungen stattfinden, wie sie bei vielen aquatischen Organismen vorkommen, oder Fortbewegung mit Hilfe von Extremitäten des Körpers, wie es bei den meisten Formen terrestrischer Lokomotion der Fall ist. Dazu bedarf es einer fein abgestimmten Koordination aller Extremitäten untereinander und damit der Koordination neuronaler Netzwerke, die lokal die motorischen Erregungsmuster zur Bewegung der jeweiligen ipsilateralen Extremität erzeugen.

Es sind dies die bei Vertebraten und vielen Invertebraten bekannten so genannten zentralen Mustergeneratoren (ZMG) im zentralen Nervensystem (ZNS), die rhythmische motorische Programme je nach Verhaltenskontext (zum Beispiel Laufoder Schwimmbewegungen) autonom erzeugen und dadurch die Bewegungen der Extremitäten steuern (unter anderen: FRIESEN and PEARCE 1993; KJAERULFF and KIEHN 1996; KIEHN and KJAERULFF 1998; ROBERTS et al. 1998; SKINNER and MULLONEY 1998a). Ein zentraler Mustergenerator besteht seinerseits meistens aus mehreren lokalen neuronalen Netzwerken in einer segmentalen Anordnung. Solche lokalen Mustergeneratoren erzeugen jeweils das motorische Programm für eine einzelne Extremität, angepasst an den jeweiligen Verhaltenskontext. Sie sind auch untereinander gekoppelt, um koordinierte Bewegungen der Extremitäten zu ermöglichen (FRIESEN and PEARCE 1993; KJAERULFF and KIEHN 1996; ROBERTS et al. 1998; SKINNER and MULLONEY 1998a). In den verschiedenen Tiergruppen gibt es jedoch große Unterschiede hinsichtlich der Eigenschaften der lokalen neuronalen Netzwerke (Oszillatoren) selbst, bezüglich ihrer Koppelung untereinander und des Einflusses von sensorischer Rückkoppelung bei ihrer Kontrolle (Review: HILL et al. 2003). So unterscheiden sich zum Beispiel die einzelnen segmentalen Oszillatoren des zentralen Mustergenerators für Schwimmen beim Egel hinsichtlich ihrer Fähigkeit, rhythmische Motorprogramme zu erzeugen (HOCKER et al. 2000). Dabei sind die hemisegmentalen Oszillatoren rhythmisch 
synchron aktiv, da die undulatorischen Schwimmbewegungen beim Egel über synchrone Kontraktionen der Dorsoventralmuskulatur in jeweils einer Segment-Hälfte erzeugt werden (HILL et al. 2003). Bei Krebsen wiederum ist bekannt, dass das gesamte neuronale Netzwerk zur Steuerung einer Extremität („swimmeret“; Pleopod) innerhalb eines Hemisegments lokalisiert ist (MURCHISON et al. 1993). Zwei hemisegmentale Oszillatoren sind hier innerhalb des Segments meistens rhythmisch synchron gekoppelt, können ihre Rhythmik aber sogar unabhängig voneinander erzeugen (MURCHISON et al. 1993).

Bei Insekten (Stabheuschrecken) gibt es Hinweise, dass sich lokale neuronale Netzwerke der Lokomotion sogar hinsichtlich ihres intersegmentalen Einflusses bei der Koppelung untereinander unterscheiden (BORGMANN et al. 2007). Zwar besteht hier ein vergleichbares Organisationsprinzip (hemisegmentale neuronale Netzwerke erzeugen autonom die rhythmischen Erregungsmuster für die jeweilige ipsilaterale Extremität), aber die Erkenntnisse sind etwas kontroverser als im Falle von Wirbeltieren (HUGHES and WIERSMA 1960; DELCOMYN 1980; HEITLER 1980; PEARSON 1985; BÄSSLER 1986, 1993; CRUSE 1990). Zwar konnte für Schaben (ROEDER 1948), Gottesanbeterinnen (ROEDER 1937) und auch für Wüsten- und Wanderheuschrecken (HUSTERT 1989) gezeigt werden, dass diese auch im dekapitierten Zustand zu koordinierten Bewegungen oder sogar Laufbewegungen (HUSTERT 1989) fähig sind. Aber es ist nicht zweifelsfrei nachgewiesen, ob motorische Erregungsmuster für verschiedene Formen von Lokomotion auch tatsächlich autonom ohne den Input von Propriozeptoren erzeugt werden können, (BERKOWITZ and LAURENT 1996), auch wenn sich "fiktive Rhythmen" an isolierten Thorakalganglien durch Zugabe von Pilocarpin in die Badlösung auslösen lassen (RYCKEBUSCH and LAURENT 1993), die sich mit solchen während natürlicher Laufbewegungen auftretenden Rhythmen vergleichen lassen. Aus diesem Grund wird im Folgenden anstelle von "zentraler Mustergenerator" die neutralere Bezeichnung „lokales neuronales Netzwerk", oder "Oszillator" im Falle von Insekten benutzt, ohne dabei eine autonome Generation motorischer Erregungsmuster im Falle von Insekten zu postulieren.

Solche neuronalen lokalen Netzwerke zur Koordination der Extremitäten sind bei Insekten in den Thorakalganglien lokalisiert und jedes Netzwerk steuert die 
Bewegung des jeweils ipsilateralen Beins (HUGHES and WIERSMA 1960; DELCOMYN 1980; HEITLER 1980; PEARSON 1985; FOTH and BÄSSLER 1985a,b; BÄSSLER 1986, 1993; CRUSE 1990). So muss jedes Bein sowohl mit dem jeweils im Segment Gegenüberliegenden koordiniert werden (bilaterale intraganglionäre Koordination der neuronalen lokalen Netzwerke) als auch mit den Extremitäten aus benachbarten Segmenten (intersegmentale Koordination). Um jedoch auch auf veränderte Bedingungen in der Umwelt bei der Lokomotion reagieren und stets ein Gleichgewicht aufrechterhalten zu können, ist sensorische Rückkoppelung über die auf Körper und Extremitäten einwirkenden Umweltreize (Exterozeption) sowie über veränderte Zustände im Körper und in den Extremitäten selbst (Propriozeption) notwendig. So werden mechanosensorische Reize beispielsweise über taktile Haare, campaniforme Sensillen, sowie auch visuell über die Ommatidien wahrgenommene optische Reize und andere an das ZNS weitergeleitet, während sensorische Informationen über veränderte Zustände im Körper und in den Extremitäten selbst beispielsweise durch Gelenkstellungs-Rezeptoren, spannungssensitive Rezeptoren, Chordotonalorgane und andere übertragen werden (BRÄUNIG et al. 1981; HUSTERT et al. 1981; PFLÜGER et al. 1981; BRÄUNIG, HUSTERT 1985a,b; BURROWS 1985; BURROWS and PFLÜGER 1988; LAURENT and HUSTERT 1988; SKORUPSKI and HUSTERT 1991; Review: NEWLAND and BURROWS 1997). Das gilt besonders für „schnelle“ Verschaltungswege von Mechanosensoren auf Motoneurone (wie zum Beispiel im Falle von einigen campaniformen Sensillen an Coxa und Trochanter der Extremitäten), die in kritischen Verhaltenskontexten sehr schnelle Reflexe ermöglichen (HÖLTJE and HUSTERT 2003). Alle lokal ablaufenden Reflexe und entsprechende ausgleichende Bewegungen der betreffenden Extremität bewirken zwangsläufig veränderte Last und Stellung aller anderen Extremitäten, was dort wiederum über lokale sensorische Rückkoppelung die Wirkungsweise der betreffenden lokalen neuronalen Netzwerke beeinflusst (über spikende- und nichtspikende lokale Interneurone, intersegmentale Interneurone und neurosekretorische Zellen; TYRER and ALTMAN 1974; PEARSON and FOURTNER 1975; ALTMAN and TYRER 1977a; BURROWS 1979b, 1980a, 1985, 1987, 1988; ALTMAN 1980; BURROWS and SIEGLER 1982; WATSON 1984; WATSON and BURROWS 1987a; NAGAYAMA and BURROWS 1990). Dies führt zur entsprechenden ausgleichenden Verlagerung oder Neupositionierung der Extremität (mechanische Koppelung). Für Stabheuschrecken wurde gezeigt, dass während Laufbewegungen eine 
intersegmentale Koordination aller Extremitäten nicht alleine über mechanische Koppelung erzielt werden kann, sondern dass auch der Austausch neuronaler Information zwischen den Netzwerken über Interneurone erforderlich ist (GRAHAM and CRUSE 1981; EPSTEIN and GRAHAM 1983). Bei der bilateralen Koordination (intrasegmental) zweier sich im Segment gegenüber liegenden Extremitäten stellt sich die Frage, ob dies zu einem großen Teil über mechanische Koppelung erfolgen kann, da eine experimentell gemessene Koppelungsstärke zwischen kontralateral benachbarten Beinpaaren nur halb so groß ist wie zwischen ipsilateral benachbarten Beinpaaren (Stabheuschrecken: DÜRR 2005). Dennoch gibt es auch Hinweise auf zentralnervöse Mechanismen zur bilateralen Koordination: So zeigen Stabheuschrecken, wenn man sie auf einer Quecksilberoberfläche laufen lässt (in starkem Maße mechanische Entkoppelung der Extremitäten beim Laufen), dennoch ein weitgehend koordiniertes Schrittmuster (GRAHAM and CRUSE 1981). Dies spricht für die Beteiligung von zentralnervösen Mechanismen bei der bilateralen Koordination. Auch von einigen Feldheuschreckenarten ist bekannt, dass die bilaterale Koordination beider Hinterbeine während der Stridulation über neuronale Verschaltungen erreicht werden muss, die einerseits über Kommissuren des Metathorakalganglions (dort, wo auch lokal in jedem Hemiganglion die neuronalen Erregungsmuster für die Stridulation erzeugt werden), andererseits über weiter anterior gelegene Kommissuren (vermutlich im Unterschlundganglion) verlaufen (HEINRICH and ELSNER 1997).

Während jeder Form der Fortbewegung, die die Koordination mehrerer Extremitäten einschließt, muss abwechselnd immer eine bestimmte Anzahl von Beinen in bestimmter Anordnung simultan gegen den Boden gestemmt werden („stance phase“), während die anderen Extremitäten die Vorwärtsbewegung des Tieres durch eine Ausholbewegung einleiten („swing phase“; Schwungphase, Neupositionierung des Beines). Nur auf diese Weise kann der Körper während der Vorwärtsbewegung kontinuierlich optimal ausbalanciert über dem Untergrund gehalten werden. Solche Fortbewegungsmuster wurden beispielsweise an Wanderheuschrecken (BURNS 1973), Stabheuschrecken (WENDLER 1964; GRAHAM 1972, 1985; Reviews: BÄSSLER 1983; CRUSE 1990), Schaben (DELCOMYN 1971a,b; FULL and TU 1990), Krabben (BURROWS and HOYLE 1973; BLICKHAN and FULL 1987), aber auch an Vögeln und Säugetieren (CAVAGNA et al. 1977; HEGLUND et al. 1982a,b) 
untersucht. Charakteristische, im Tierreich - vor allem bei Insekten - verbreitete Schrittmuster in diesem Zusammenhang sind die Dreipunkt- (tripod gait) und die Vierpunkt- Unterstützung (tetrapod gait) des Körpers, bei der in abwechselnder Weise immer jeweils die entsprechende Zahl von Beinen (drei oder vier) zeitgleich den Körper unterstützen. Wüsten- und Wanderheuschrecken nutzen während der Fortbewegung bei unterschiedlichen Geschwindigkeiten den tripod gait (DreipunktUnterstützung), obschon bei innen wohl auch aufgrund der Spezialisierung der Hinterbeine für bestimmte Verhaltensweisen (Sprung) die größte innerartliche Variabilität bezüglich der Beinbewegungen bei diesem vorherrschenden Schrittmuster auftritt (BURNS 1973).

Für Wüsten- und Wanderheuschrecken wurden bisher einige intersegmentale Interneurone beschrieben, die Eigenschaften einer die Extremitäten während der verschiedensten Verhaltensweisen koordinierenden Funktion haben (WATSON and BURROWS 1983; RAMIREZ and PEARSON 1988; LAURENT and BURROWS 1989a,b). Aber nur bei einer Gruppe von intersegmentalen Interneuronen weiß man, wie sie im Detail verschalten. Neurone dieses Typ haben Somata, die posteriorventral im Mesothorakalganglion lokalisiert sind und deren Axon im ipsilateralen Konnektiv absteigt und ins Metathorakalganglion projiziert (LAURENT 1987). Sie verschalten sowohl direkt auf Motoneurone als auch auf nicht-spikende lokale Interneurone des lokalen Netzwerkes, das die Bewegungen des ipsilateralen Hinterbeins kontrolliert (LAURENT and BURROWS 1989b). Die Mehrzahl der intersegmentalen Interneurone dieser Gruppe (etwa 80\%) verschalten erregend auf metathorakale Neurone. Bislang wurden jedoch noch keine rein bilateral koordinierenden Interneurone identifiziert.

Dennoch bieten Wanderheuschrecken günstige Voraussetzungen zum Studium der bilateralen Koordination. Eine Vielzahl von Motoneuronen und Interneuronen in den Thorakalganglien, die jeweils in Gruppen von morphologisch ähnlichen Neuronen nahe beieinander lokalisiert sind, sind identifiziert. Anhand der Lage ihrer Somata wurden so genannte „neuronale Karten“ erstellt (Motoneurone: BENTLEY 1970; BURROWS and HOYLE 1973; TYRER and ALTMAN 1974; WILSON 1979; Interneurone: BURROWS and SIEGLER 1984; SIEGLER and BURROWS 1979, 1984; WILSON 1981; WILSON and PHILLIPS 1982), mit Hilfe derer sich bislang 
unbekannte Neurone leichter klassifizieren oder gegebenenfalls einer bestehenden Klasse zuordnen lassen. Außerdem sind die zentralen Projektionsbereiche einer Vielzahl von Mechanosensoren (Extero- wie Propriozeptoren) in die entsprechenden Neuropilregionen im Detail bekannt. Neben weiteren seien hier tastsensitive Haare und Haarfelder („hair plates“: HUSTERT 1978; PFLÜGER 1980; PFLÜGER et al. 1981), zervikale Haarfelder (KIEN 1980), campaniforme Sensillen (HUSTERT 1978; HUSTERT et al. 1981), Chordotonalorgane (HUSTERT 1978; BRÄUNIG et al. 1981; BURROWS 1987b) und spannungssensitive Rezeptoren in den Extremitäten (BRÄUNIG and HUSTERT 1980; BRÄUNIG 1982a,b) genannt. Dabei projizieren nur wenige propriozeptive Afferenzen bilateral in das kontralaterale Hemiganglion (HUSTERT 1978), was wiederum für eine bilaterale Koordination zweier sich gegenüber liegenden Extremitäten über kommissurale Interneurone spricht.

Die wichtigsten morphologischen Strukturen wie Trakte (longitudinal, vertikal und andere), Kommissuren und Neuropilregionen und ihre Lokalisation in den Thorakalganglien sind ebenso bekannt und in verschiedenen Übersichten in einigen Arbeiten umfassend dargestellt (PFLÜGER et al. 1988; PIPA et al. 1959; GREGORY 1974, 1985; TYRER and GREGORY 1982). Diese Grundlagen erleichtern Analysen der Morphologie von Interneuronen, da sich ihre Verzweigungen so in Relation mit solchen Strukturen beschreiben lassen. Dabei sind vor allem die lateralen (dorsal gelegenen) und die ventralen Neuropilregionen von Interesse, da unter anderem ein Großteil der Mechanosensoren in diese Bereiche projiziert. So ist bekannt, dass alle taktilen (tastsensitiven) Haare in die ventralen Neuropile projizieren (PFLÜGER 1980; PFLÜGER et al. 1981). Letztere sollen auch größtenteils aus sensorischen Fasern bestehen (PIPA et al. 1959; GREGORY 1974; ALTMAN 1980; PFLÜGER et al. 1981), wenn auch manche intersegmentale Interneurone (MURPHEY et al. 1983, 1985; PFLÜGER 1984; HUSTERT 1985) und lokale Interneurone (BURROWS and SIEGLER 1984; SIEGLER and BURROWS 1984) in diesen ventralen Neuropilen verzweigen. In den lateralen Neuropilregionen kommen sowohl sensorische Fasern als auch Fasern von Moto-, Interneuronen und Fasern von neurosekretorischen Zellen vor (TYRER and ALTMAN 1974; ALTMAN and TYRER 1977; ALTMAN 1980; WATSON 1984). Besonders in diesen lateralen Neuropilen sind Verzweigungen von bilateral koordinierenden kommissuralen Interneuronen zu erwarten, weil diese hier zum einen direkt auf Motoneurone verschalten könnten, zum anderen, weil sie hier den im Kontext der bilateralen Koordination relevanten sensorischen Input (entweder 
direkt über Propriozeptoren, oder indirekt über sensorisch integrierende lokale Interneurone) erhalten könnten. Nichtsdestotrotz ist über die bilaterale Koordination der Extremitäten bei Wüsten- und Wanderheuschrecken wenig bekannt. HUSTERT (1989) hat ein intersegmentales, kommissurales Interneuron im Mesothorakalganglion von Schistocerca gregaria beschrieben, das Eigenschaften einer die Extremitäten koordinierenden Funktion hat. Das Neuron verzweigt bilateral und hat ein dorsal-lateral gelegenes Soma. Das Axon projiziert kontralateral in das Pro- und Metathorakalganglion (Bifurkation). Es reagiert phasisch erregt während aktiver und passiver Levation des ipsilateral zum Soma gelegenen Mittelbeins. Bei kontralateraler Levation hingegen wird das Neuron in dieser Phase gehemmt. Da das Neuron Input vom ipsilateralen Mittelbein zu erhalten scheint und kontralateral in das Pro-, und Metathorakalganglion projiziert, ist anzunehmen, dass es auch die motorische Kontrolle vom kontralateralen Vorder- und Hinterbein im Kontext der Aufrechterhaltung eines tripod gait beeinflusst (HUSTERT 1989).

Untersuchungen an Neunaugen (BIRÓ et al. 2006, 2008), Krebsen (MULLONEY and HALL 2003), Nagetieren (BUTT et al. 2002; BUTT and KIEHN 2003; LANUZA et al. 2004; QUINLAN and KIEHN 2007) und am Krallenfrosch (SOFFE et al. 1984) haben gezeigt, dass kommissurale Interneurone eine wichtige Rolle bei der bilateralen Koordination der Extremitäten bzw. Körperpartien spielen. Auch die synchronen rhythmischen Erregungsmuster hemisegmentaler Oszillatoren beim Schwimmen des Egels werden auf ihre starke elektrische und chemische Koppelung (intrasegmental, über die Mittellinie hinweg) zurückgeführt (FRIESEN and HOCKER 2001). An isolierten (also deafferentierten und deefferentierten) Präparaten vom Rückenmark des Neunauges („spinalisierter Fisch“) können durch Zugabe von exzitatorisch wirkenden Agenzien (wie beispielsweise Glutamat) in die Badlösung rhythmische Aktivitäten in den auch für die Schwimmbewegungen verantwortlichen neuronalen Netzwerken ausgelöst werden (COHEN and WALLÉN 1980; COHEN et al., 1992). $\mathrm{Da}$ das Muster dieser in vitro erzeugten rhythmischen Aktivitäten dem während natürlichen Schwimmbewegungen auftretenden hinsichtlich der bilateralen wie intersegmentalen Koordination sehr ähnlich ist, wird es als "fiktives Schwimmen“ bezeichnet (COHEN and WALLÉN 1980; WALLÉN and WILLIAMS 1984). Die undulatorischen Schwimmbewegungen beim Neunauge werden durch alternierende Kontraktionen von Muskelpartien der Körperflanken erzeugt. Zur Erzeugung eines 
alternierenden rhythmischen Erregungsmusters ist jeweils ein ipsilateraler hemisegmentaler Mustergenerator reziprok mit dem korrespondierenden kontralateralen Oszillator verschaltet (BUCHANAN 1986; BUCHANAN and GRILLNER 1987). Diese reziprok gerichtete Koppelung, die hauptsächlich inhibitorischer Art ist (es gibt auch noch erregende Verschaltungen zwischen den Oszillatoren), wird auf kommissurale Interneurone (CIN: commissural interneuron) zurückgeführt, die (zumindest im Falle von Wirbeltieren) durch eine ventrale Kommissur verlaufen (BUCHANAN 1986; BUCHANAN and GRILLNER 1987; BIRÓ et al. 2008). Durch gegenseitige abwechselnde Hemmung der beiden lokalen Mustergeneratoren untereinander kommen letztlich die rhythmisch alternierenden motorischen Aktivitäten zustande (BUCHANAN 1999). An Neunaugen wurde bislang eine Klasse von kommissuralen Interneuronen (CCIN) beschrieben, deren Axon nach kontralateral und caudal projiziert und die eine solche reziproke Inhibition vermitteln (BUCHANAN and COHEN 1982). Neuere Ansätze haben jedoch Modelle beschrieben, in welchen die hemisegmentalen Netzwerke auch unabhängig voneinander oszillieren können und in welchen der Einfluss der reziproken Hemmung auf die Rhythmik weniger stark ist (HELLGREN-KOTALESKI et al. 1999).

Auch am isolierten Rückenmark von neonatalen Nagetieren wie Ratten und Mäusen werden durch exzitatorisch wirkende Pharmaka wie beispielsweise NMDA oder Serotonin (5-HT) „fiktive“ Bewegungsmuster ausgelöst, um Lokalisation und Funktionsweise des für Generation und Koordination von Lokomotion zuständigen neuronalen Netzwerkes zu untersuchen (CAZALETS et al. 1992, 1995; KJAERULFF and KIEHN 1996; BRANCHEREAU et al. 2000; ZHONG et al. 2006b). Kommissuralen Interneuronen werden auch hier eine wichtige Rolle bezüglich der rechts-links-Koordination (bilaterale Koordination) zugeschrieben (BUTT et al. 2002; BUTT and KIEHN 2003; LANUZA et al. 2004; QUINLAN and KIEHN 2007). Es wird ebenfalls angenommen, dass - ähnlich wie beim Neunauge und beim Krallenfrosch direkte reziprok gerichtete synaptische Verbindungen zwischen kommissuralen Interneuronen in korrespondierenden gegenüberliegenden Hemisegmenten des Rückenmarks bestehen (BIRINYI et al. 2003).

Bisher wurden an Wirbeltieren vier Klassen kommissuraler Interneurone (CIN) beschrieben (EIDE et al. 1999; STOKKE et al. 2002; NISSEN et al. 2005; MATSUYAMA et al. 2006): Absteigende (dCINs, descending), aufsteigende 
kommissurale Interneurone (aCINs, ascending), solche mit Bifurkation (adCINs, ascending-descending; kontralateral im Rückenmark „durchlaufend“, also sowohl auf- als auch absteigend), sowie Neurone (sCINs, segmental CINs, oder auch „short range“ CINs), die die Mittellinie über eine Kommissur durchqueren, aber deren axonaler Projektionsbereich sich in der Regel nur über kürzere Distanzen erstreckt. Auch „short range“ CINs können intersegmental verzweigen, dann erstrecken sie sich allerdings höchstens über eine Strecke von maximal 1,5 Segmenten. Bei den meisten Neuronen dieser Klasse handelt es sich aber um lokale, auf ein Segment beschränkte kommissurale Interneurone (BIRINYI et al. 2003). Die ersten drei genannten Klassen (aCINs, dCINs und adCINs) werden auch unter der Bezeichnung „long range“ CINs zusammengefasst. Sowohl „short range“ als auch „long range“ CINs sollen an der bilateralen Koordination zwischen korrespondierenden Mustergeneratoren beteiligt sein (QUINLAN and KIEHN 2007).

Von den „short-range“ CINs ist bekannt, dass sie direkt und indirekt auf Motoneurone kontralateral gelegener Gruppen (Motoneuron-,pools“) innerhalb des Segmentes verschalten (QUINLAN and KIEHN 2007). Dabei gibt es neben einer erregend wirkenden direkten Verschaltung auf Motoneurone auch noch zwei weitere Verschaltungswege hemmender Art. Eine davon ist eine direkt hemmende Verschaltung auf Motoneurone, bei der anderen wird vom betreffenden SCIN ein inhibitorisches Interneuron aktiviert, das seinerseits Motoneurone hemmt (indirekt hemmende Verschaltung).

Die "short range“ CINs haben Verzweigungsbereiche, die kontralateral in der Inputregion von Motoneuron-Populationen liegen, sowie solche, die in einem ventralmedianen Bereich liegen (HOOVER and DURKOVIC, 1992; BIRINYI et al. 2003; MATSUYAMA et al. 2006). Alle Neurone dieser sCIN-Klasse machen einen eher geringen Anteil (10-15\%) an der Gesamtpopulation kommissuraler Interneurone aus (STOKKE et al. 2002). Ihre direkte Verschaltung auf Motoneurone allein scheint jedoch noch nicht ausreichend für eine bilaterale rechts-links-Koordination im Kontext von laufartigen Bewegungen zu sein (QUINLAN and KIEHN 2007). Daneben werden auch zusätzliche Verschaltungen auf andere Neurone postuliert, die Teil des für die Laufbewegungen verantwortlichen zentralen Mustergenerators sind (QUINLAN and KIEHN 2007). So sind bereits synaptische Verbindungen zwischen ipsi- und kontralateralen kommissuralen Interneuronen bekannt (BIRINYI et al. 2003; MATSUYAMA et al. 2006). 
Unter den „Iong range“ CINs werden vor allem solchen mit absteigendem Axon (dCINs) eine bilateral koordinierende Funktion im Kontext von Bewegung zugeschrieben. Auch bei diesen gibt es direkt erregende und direkt oder indirekt hemmende Verschaltungen (BUTT and KIEHN 2003; QUINLAN and KIEHN 2007). Sie sollen an der bilateralen wie intersegmentalen Koordination von während der fiktiven Bewegungsmuster synergistisch wirkenden Flexor- und Extensormuskeln beteiligt sein (BUTT et al. 2002; BANNATYNE et al. 2003; BUTT and KIEHN 2003; JANKOWSKA et al. 2003, 2005; KRUTKI et al. 2003; EDGLEY et al. 2004; HAMMAR et al. 2004). Aber auch bei den aufsteigenden kommissuralen Interneuronen (aCINs) geht man von einer bilateral koordinierenden Funktion im Kontext von fiktiver Lokomotion aus, da sie während solcher Bewegungssequenzen rhythmisch aktiv sind (CARLIN et al. 2006; ZHONG et al. 2006a,b) und indirekt hemmend auf kontralaterale Motoneurone verschalten (QUINLAN and KIEHN 2007).

Es ist noch nicht genau bekannt, welche Rolle die kommissuralen Interneurone, deren Axon sowohl auf- als auch absteigt (adCINs), bei der Koordination der Extremitäten spielen. Zwar sind auch bei innen indirekt inhibitorische Verschaltungen auf kontralaterale Motoneurone nachgewiesen worden (QUINLAN and KIEHN 2007), jedoch wurden bisher keine solchen Neurone gefunden, die im Kontext der fiktiven Bewegungsmuster rhythmische Aktivität zeigten (ZHONG et al. 2006b). Es wird vermutet, dass solche Neurone eine wichtige Rolle im Kontext von nichtrhythmischen Bewegungen (Reflexen) spielen könnten (ZHONG et al. 2006b).

Überdies wird angenommen, dass vor allem die hemmend wirkenden kommissuralen Interneurone wie auch im Falle von Neunaugen (BUCHANAN 1986; BUCHANAN and GRILLNER 1987) und vom Krallenfrosch (SOFFE et al. 1984) wichtig bei der Generation des bilateral alternierend auftretenden rhythmischen Motorprogramms (reziproke Inhibition zwischen bilateralen lokalen Netzwerken) sind (QUINLAN and KIEHN 2007). Noch nicht genau geklärt ist die Frage, welche Rolle die erregend wirkenden kommissuralen Interneuronen bei der Koordination von Bewegung spielen. So könnten sie entweder im Kontext von alternierenden Bewegungen (laufartigen Bewegungen) oder während synchroner Bewegungen (hüpfende Bewegungen, Springen) aktiv sein (QUINLAN and KIEHN 2007).

Die vorliegende Arbeit soll einen Beitrag zur Klärung der Frage nach neuronalen Mechanismen der bilateralen Koordination der Extremitäten bei Insekten leisten, da 
dies hier noch weitgehend unbekannt ist. An einem semi-intakten ventral gehalterten Präparat (Kopf/Thorax/Abdomen; Präparation nach HUSTERT, 1989; leicht modifiziert) mit frei auf einer Laufkugel-Konstruktion beweglichen Vorder- und Mittelbeinen können durch taktile Reize an der Dorsalseite eines Mittelbein-Tarsus bilaterale und intersegmentale Reflexe zwischen den Extremitäten ausgelöst werden. Solch eine Reizung löst einen ipsilateralen (lokalen) Reflex aus („Treading on tarsus“-Reflex, „TOT-Reflex“: GRAHAM, 1979; SCHMITZ and HAßFELD 1989), bei welchem das gereizte Bein kurzzeitig angehoben wird (Kontraktion der Muskeln: Levator tarsi, Levator trochanteris, Extensor tibiae; nach SNODGRASS 1929) und das kontralaterale aufgrund veränderter Lastverhältnisse stärker gegen den Untergrund gestemmt wird (Kontraktion des kontralateralen Depressor trochanterisMuskel). Ein bilateral koordinierendes Interneuron könnte im einfachsten Fall intraganglionär bilateral verzweigen, wobei es in einem Hemiganglion sensorischen Input über den ipsilateral ablaufenden Reflex (zum Beispiel durch Reizung ausgelöste Levation; Levator trochanteris aktiv) erhält und im gegenüber liegenden Hemiganglion beispielsweise direkt erregend auf Motoneurone des Depressor trochanteris verschaltet (Lastkompensation; bilateraler Reflex). Eine durch Reizung ausgelöste Levation des kontralateralen Beins sollte bei einem solchen Verschaltungsprinzip des Interneurons hingegen zu seiner Hemmung während des ablaufenden kontralateralen lokalen Reflexes führen, wenn es erregend auf Motoneurone des kontralateralen Depressormuskels verschaltet und bei diesem Reflex der antagonistische Levator trochanteris aktiviert wird (HUSTERT 1989). Die intrazellulär im Bereich der dorsalen Kommissuren abgeleiteten Aktivitäten mesothorakaler Interneurone lassen sich in zeitliche Relation zu parallel extrazellulär abgeleiteten Muskelaktivitäten vom Depressor trochanteris beider Mittelbeine setzen, um ihre potentiell bilateral die Extremitäten koordinierende Funktion zu analysieren. In der vorliegenden Arbeit werden nun kommissurale Interneurone im Mesothorakalganglion von Locusta migratoria migratorioides vorgestellt, welche Eigenschaften einer die Extremitäten bilateral koordinierenden Funktion haben können. Nur solche Neurone können eine neurale bilaterale Koordination zwischen zwei sich im Segment gegenüberliegenden Extremitäten leisten. 


\subsection{Auswahl der Versuchstiere}

Für die Experimente wurden adulte weibliche und männliche Exemplare von Locusta migratoria verwendet, die der institutseigenen Kultur entstammen. Die Tiere werden in einem im zwölf Stunden Rhythmus wechselnden Hell-Dunkel-Zyklus bei $30^{\circ} \mathrm{Grad}$ Raumtemperatur gehalten. Es wurden bevorzugt Exemplare ausgewählt, bei welchen die letzte Häutung etwa ein bis zwei Wochen zurückliegt. Zu diesem Zeitpunkt besitzt die Kutikula einerseits eine für die spätere Fixierung der Präparate ausreichende Festigkeit. Andererseits ist bei entsprechenden Tieren die Dorsoventral- und Flugmuskulatur noch nicht vollständig entwickelt, was das Freilegen und die Präparation der Thorakalganglien erheblich erleichtert. Chronische Muskelableitungen (Myogramme) von beiden Depressor trochanteris Muskeln (M103a, nach SNODGRASS 1929) konnten bei diesen Tieren zuverlässig an beiden Mittelbeinen vorgenommen werden. Da für den Versuch semi-reduzierte Thorax/Kopf-Präparate der Heuschrecken erstellt werden mussten, waren lebhafte Tiere mit einem ausgeprägten Fluchtverhalten vorteilhaft. Bei ihnen war die Wahrscheinlichkeit größer, dass sie auch nach der die Vitalität des Tieres beeinträchtigenden Präparation Laufbewegungen zeigen konnten.

2.2 Konstruktion einer rotierbaren Laufvorrichtung für gehalterte Heuschrecken unter den Bedingungen der elektrophysiologischen intrazellulären Ableitung

Bei den durch intrazelluläre Ableitung untersuchten neuronalen Eigenschaften während ausgelöster bilateraler und intersegmentaler Reflexe sollte auch das neuronale Verhalten während aktiver Bewegung oder sogar Laufbewegungen studiert werden. In anfänglichen Experimenten wurden die ventral gehalterten Präparate auf einer luftgelagerten Styroporkugel positioniert. Diese erwies sich jedoch insofern als nachteilig, als dass sich die Tiere gelegentlich bei taktiler Reizung verschiedener Art gegen den Untergrund zu stemmen versuchten. Dabei arretierte die Styroporkugel innerhalb der Kunststoffschale und es kam in der Regel zum 



Abb. 1: A-B: Konstruktion für eine leicht rotierbare Laufkugel aus Styropor. Seitliche Ansicht (A) und Aufsicht (B) auf die Konstruktion. Die Styroporkugel (L) ist um eine Achse rotierbar, die aus zwei Insektennadeln $(\mathrm{N})$ gebildet wird. Die Nadeln sind in abgerundeten Glashülsen und diese wiederum in Holzblöckchen gelagert (BL). Die Konstruktion wird durch einen Kunststoffring (SR) getragen und ist über Gummifäden $(G)$, die über Gelenkrollen $(R)$ von drei Stützdrähten $(S)$ laufen, elastisch aufgehängt (vertikale Pfeile in A). Die Stützdrähte werden durch einen Holzblock $(\mathrm{Hz}$, zwei untereinanderliegende Dreieck-Holzscheiben) stabilisiert. An diesem sind auch die Gummifäden über Ösenschrauben befestigt $(\mathrm{H})$. Der Holzblock - und damit die gesamte Konstruktion - ist auf einem drehbaren Untersatz angebracht, in diesem Fall ein ausrangierter Computerlüfter (VN). Aufgrund seiner leichten Drehbarkeit in der Horizontalen ist eine Änderung der Laufrichtung bei dem gehalterten Präparat ohne weiteres möglich. B: Aufsicht auf das ventral gehalterte Präparat und die Laufkugel. Die Halterung $(\mathrm{HT})$ ist ein Metallträger, der am vorderen Ende abgeflacht und mit California Red Wachs (Dentalwachs) abgedeckt ist, zur Verhinderung einer elektrisch leitenden Verbindung zwischen Präparat und Peripherie. Thorax und Abdomen des Tieres sind dorsal eröffnet. Teile der Dorsoventralmuskulatur (M), Fett- und Nährgewebe (F) und Tracheen (TA) sind soweit entfernt, dass die Thorakalganglien des Zentralnervensystems (hier abgebildet: Meso- und Metathorakalganglion, T2 und T3) frei liegen. Das Mesothorakalganglion (T2) ist auf einer Metallplattform (,Löffel“, LF) gelagert, die gleichzeitig als Erdung des Präparates dient. Die Glasmikroelektrode (I) wird in den Bereich der dorsalen Kommissuren des Mesothorakalganglions senkrecht eingestochen. Die Elektrode ist hier aus Gründen der Darstellung schräg angeordnet. Der Kopf des Tieres ist über zwei Wachsbrücken (W) mit dem Thorax verbunden und fixiert. Die Myogrammdrähte der Muskelableitungen vom Depressor trochanteris vom rechten (DR) und linken (DL) Mittelbein können nach hinten geführt werden, da die Hinterbeine bei diesem Präparat entfernt werden.

Abkürzungen: BL: Holzblöckchen mit integrierter Glashülse zur Aufnahme der Achsen-Nadeln (N); DL-DR: Myogrammelektroden, Depressor trochanteris, rechtes (DR) und linkes (DL) Mittelbein; F: Fett- und Nährgewebe im Abdomen des Präparates; G: Gummifaden; $\mathrm{H}$ : Halterung für Gummifaden (Ösenschraube); HZ: Holzblock, Stabilisierung der Laufkugel-Konstruktion; I: Intrazelluläre Ableitung (Glasmikroelektrode); L: Styroporkugel $(\varnothing=55 \mathrm{~mm})$; M: Muskelgewebe im Präparat, nicht differenziert, im Wesentlichen Dorsoventral- und Flugmuskulatur; N: Achsennadeln aus Insektennadeln; R: Gelenkrolle aus Glashülse; S: Stützdraht inklusive Drahthalterung für eine Gelenkrolle; TA: Trachee (Tracheenstamm); T2: Mesothorakalganglion; T3: Metathorakalganglion; VN: Leicht drehbarer Untersatz für Konstruktion (Computerlüfter); W: Stabilisierung, Kopf über Wachsbrücken am Thorax festgelegt. 
Verlust der intrazellulären Ableitung. Aus diesem Grund wird hier eine andere Konstruktion vorgestellt, bei welcher ebenfalls eine in allen Richtungen leicht drehbzw. rotierbare Styroporkugel zum Einsatz kommt, wobei die gesamte Konstruktion aber bei derartigen Stemmbewegungen elastisch nachgibt (Abb. 1, A-B). Die rotierbare Achse der Styroporkugel (Durchmesser: 55mm) ist durch zwei herausstehende Insektennadeln (Sphinx; Größe: 3, Länge: 38mm, Stärke: 0,5mm) vorgegeben, deren Spitzen jeweils in einer an einem Ende abgerundeten Glashülse (Bastelbedarf; Durchmesser: 1,5mm (außen), 0,7mm (innen); Länge: $6 \mathrm{~mm}$ ) rotierbar gelagert sind. Dadurch wird eine sehr leichtgängige Rotation aufgrund geringer Reibung ermöglicht. Die Glashülsen sind in jeweils einem kleinen Holzblöckchen (Sperrholz; Länge: 25mm, Breite: $10 \mathrm{~mm}$, Höhe $5 \mathrm{~mm}$ ) versenkt. Letztere wiederum sind auf einem stabilisierenden Kunststoffring (Bastelbedarf; Durchmesser: 75mm (innen), 100mm (außen), Höhe: 10mm), der die Kugel umgibt, befestigt. An einem Stück aus Sperrholz in Form eines gleichseitigen Dreiecks (Höhe: 8mm, Kantenlänge: $65 \mathrm{~mm}$ ) ist an jeder Spitze jeweils ein senkrecht stehender Stützdraht (Messing; Stärke 2mm, Länge $50 \mathrm{~mm}$ ) in das Holz eingearbeitet und verklebt. Die Stützdrähte dienen zur Aufhängung der Laufkugelkonstruktion und stehen vertikal zwischen Styroporkugel und Kunststoffring, ohne diese zu berühren. An jeder oberen Spitze eines Stützdrahtes ist jeweils eine dreieckförmige Drahtkonstruktion (gebogen aus einer Sphinx Insektennadel; Gr.3, Länge 38mm, Stärke: 0,5mm) über verklebte Kupferdrahtwicklungen befestigt, die eine nach außen weisende und über die horizontale Achse rotierbare Gelenkrolle (Glashülse aus dem Bastelbedarf; Durchmesser: 1,5mm (außen), 0,7mm (innen), Länge: $6 \mathrm{~mm}$ ) trägt. Über jede dieser Gelenkrollen gleitet jeweils ein Gummizug (weicher Gummifaden von möglichst dünnem Durchmesser, Bastelbedarf), der an einem Ende am Kunststoffring befestigt ist (durch ein Bohrloch hindurch gezogen und am anderen Ende verknotet). Jeder Gummifaden wird vom Kunststoffring über die jeweilige Gelenkrolle gelegt, dann senkrecht nach unten entlang der Stützdrähte geführt und genau wie selbige über die Ecken des dreieckigen Holzstückes gezogen. Die Befestigung der Gummis erfolgt


Holzdreieck (Höhe: $8 \mathrm{~mm}$, Kantenlänge: $35 \mathrm{~mm}$ ) verankert sind. Letzteres liegt unterhalb des größeren Holzstückes um $60^{\circ}$ versetzt und ist mit diesem verklebt. Beide zusammen bilden den Standfuß der Konstruktion. Damit bei einem laufenden Tier unter gehalterten Bedingungen trotzdem eine Änderung der Laufrichtung 
ermöglicht wird, muss eine Rotation der gesamten Konstruktion in der horizontalen Ebene gewährleistet sein. Dafür wurde der Standfuß der Konstruktion ( $\mathrm{Hz}$ : beide Holzdreiecke) auf einen leicht drehbaren Untersatz aufgeklebt. Hierzu kann zum Beispiel ein nicht mehr benutzter kleinerer Computerlüfter (Hersteller nicht bekannt, Maße 50x50x25mm, vergleichbares Modell: Papst 512 F2 / 3-Pin Anschluss) dienen, wie in Abbildung 1, A-B gezeigt. Die Rotationsfähigkeit der Konstruktion ist zwar nicht nahezu reibungslos wie bei einer luftgelagerten Styroporkugel, aber in der Praxis immer noch leichtgängig genug. Ein großer Vorteil ist die elastische Aufhängung der Konstruktion. Zwar können Verluste der intrazellulären Ableitungen auch hiermit nicht gänzlich verhindert, aber zumindest deutlich reduziert werden.

Abgesehen von Insektennadeln und drehbarem Untersatz (zum Beispiel PCGehäuselüfter) sind alle Materialien im Baumarkt oder in Geschäften für Bastelbedarf preisgünstig erhältlich. Statt Insektennadeln können ersatzweise auch andere Metallnadeln beziehungsweise Drähte (Bastelbedarf) verwendet werden, wenn sie den Anforderungen an die Stabilität genügen.

\subsection{Präparation}

2.3.1 Chronische Muskelableitungen vom Depressor trochanteris (M103a) beider Mittelbeine

Von einer durch Kühlung bei $+4^{\circ}$ Celsius immobilisierten Heuschrecke wurden zunächst beide Hinterbeine oberhalb der Coxen abgetrennt, ebenso wie beide Vorder- und Hinterflügel. Das Präparat wurde dann mit der Ventralseite nach oben in einer Präparationswanne mit Knetmasse fixiert. Bei den Mittelbeinen war darauf zu achten, dass die Ansatzstellen der Depressormuskeln (M103a, Abb. 2, A,B) äußerlich sichtbar und zugänglich blieben. Die Schnittstellen oberhalb der Coxen der beiden Hinterbeine wurden umgehend mit einem Bienenwachs-KolophoniumGemisch (Verhältnis 1:1) dicht verschlossen. Ebenso wurden alle Sterniten mit Wachs bedeckt, so dass möglichst alle Haare in diesem Bereich im Wachs eingeschlossen waren. Dies war für die spätere Fixierung der Präparate nötig sowie zur Minimierung des sensorischen Inputs aus diesem Feld. Um die Beweglichkeit der Coxen der Hinterbeine zu unterbinden wurden diese leicht nach caudal ragend 

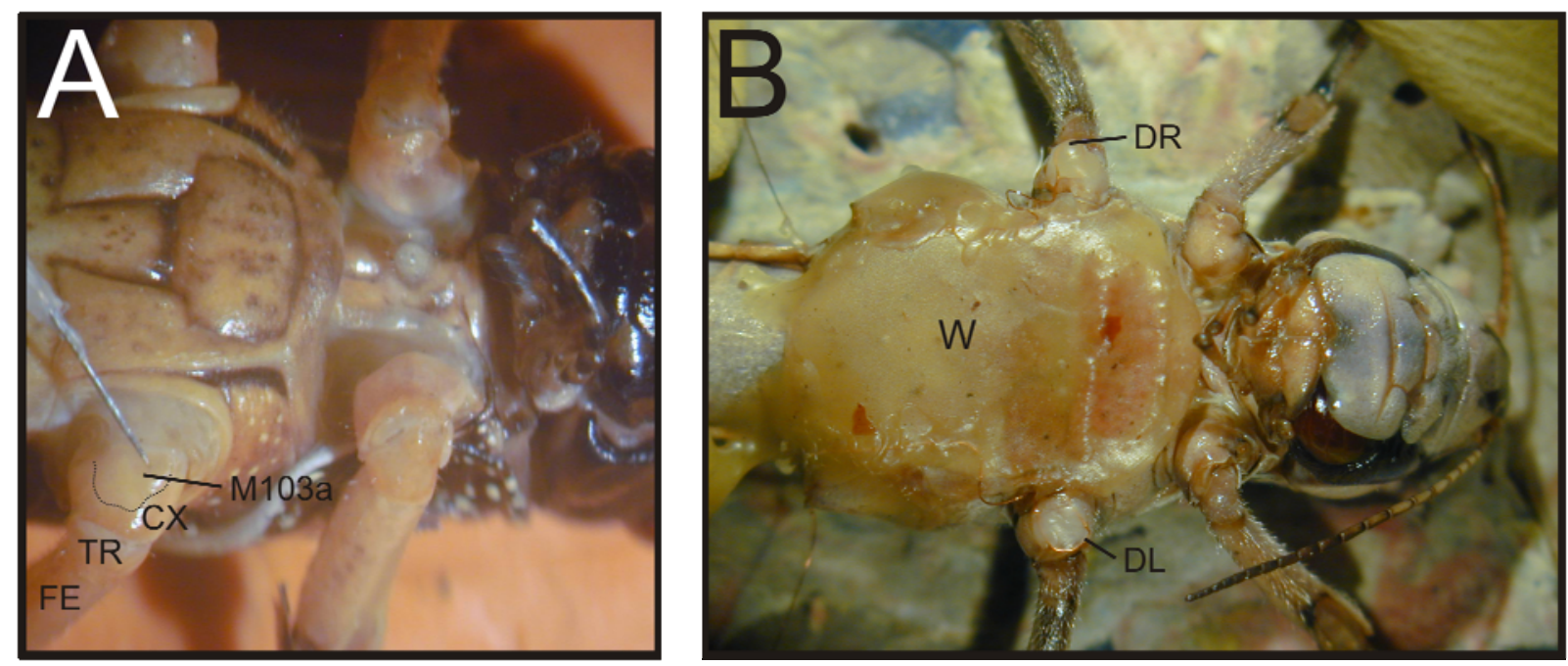

Abb. 2: A: Aufsicht auf die Ventralseite des Präparates vor Implantierung der Drahtelektroden für die Muskelableitungen. Das Bild zeigt die Muskelansatzstelle des Depressor trochanteris (M103a) an der Coxa (CX) des linken Mittelbeins. B: Aufsicht auf die Ventralseite des Präparates mit je einer implantierten Myogrammelektrode in der Coxa vom rechten (DR) und linken (DL) Mittelbein. Die Hinterbeine wurden oberhalb der Coxen abgetrennt, die Schnittflächen mit Wachs (W) verschlossen, ebenso wie das Sternum und die auf inm befindlichen Haare.

Abkürzungen: CX: Coxa (linkes Mittelbein); DL-DR: Myogrammelektroden für Muskelableitungen vom Depressor trochanteris, rechtes (DR) und linkes (DL) Mittelbein; FE: Femur (linkes Mittelbein); M103a: Depressor trochanteris, Ansatzstelle an der Coxa durch gestrichelte Linie angedeutet; TR: Trochanter (linkes Mittelbein); W: Wachsabdeckung des Sternums.

seitlich am Thorax mit Wachs fixiert (Abb. 2, B). An jedem Mittelbein wurde jeweils an der Ansatzstelle des Depressors mittels einer feinen Minutien-Nadel ein kleines Loch in die Kutikula gestochen. Von einem lackisolierten Kupferdraht (ElektrisolaEckenhagen; Cu-Lackdraht, $60 \mu \mathrm{m}$ Außendurchmesser) wurde die oberste Spitze abgeschnitten. Der Draht wurde mit einer feinen Pinzette durch das vorbereitete Loch in der Coxa eingeführt, in eine leicht auf dem Muskel aufsitzende Position gebracht und anschließend mit Wachs-Kolophonium auf der Coxa fixiert. Alle zur Ableitung implantierten Kupferdrähte wurden nun lateral am Sternum befestigt, indem die dort bereits aufgetragene und erkaltete Wachsschicht auf Höhe des jeweils ipsilateralen Mittelbeins mit einem Lötkolben (Voltcraft; Modell 2256, 1,5-15V DC Power Supply) kurz erhitzt und der Draht mit der Pinzette hineingezogen wurde. Von dort wurden die Drähte jeweils weiterhin lateral am Sternum entlang in den Wachs eingelassen, nach posterior geführt und am Ansatz des Abdomens lateral vorbei in Richtung der Dorsalseite geführt. Über die gesamte Distanz der Drahtführungen war es wichtig, dass die Drahtelektroden an allen beweglichen Stellen - insbesondere der Extremitäten - in ausreichend großen Schlaufen geführt 
wurden, um einerseits größtmögliche Bewegungsfreiheit zu gewährleisten, wobei aber andererseits sichergestellt sein musste, dass sich die Tiere nicht so leicht mit den Tarsi in solch einer Schlaufe verfangen konnten. Aus diesem Grund wurden alle Schlaufen nach posterior weisend und lateral flach am Thorax (Pleurum) anliegend fixiert (Abb. 2, B). Auf das Implantieren einer indifferenten Drahtelektrode konnte verzichtet werden, weil eine Erdung später kombiniert für Muskelableitungen und intrazelluläre Neuronableitung über eine Metallplattform (Abb. 1, B und Abb. 3, D1 und D2D) erfolgte, die zur Festlegung des Mesothorakalganglions benutzt wurde. Der Präparationsabschnitt für die chronischen Muskelableitungen von beiden Depressormuskeln (Depressor trochanteris, M103a, nach SNODGRASS 1929) war an dieser Stelle abgeschlossen.

2.3.2 Präparation eines semi-reduzierten Kopf-/Thorax-/Abdomen-Präparates für intrazelluläre Ableitungen von Interneuronen des Mesothorakalganglions

Nach Anbringung der Kupferelektroden für die chronischen Muskelableitungen (Elektromyogramm, EMG), wie im vorigen Abschnitt beschrieben, wurde das Tier aus der Präparationswanne herausgenommen. Die Präparation erfolgte nach HUSTERT (1989), mit dem einzigen Unterschied, dass der Kopf am Präparat belassen wurde. Nach einigen Experimenten hatte sich herausgestellt, dass sich an solchen Präparaten Reflexe zuverlässiger auslösen ließen und dass auch spontane, rhythmische Bewegungssequenzen (vergleichbar zum Laufen) mit größerer Wahrscheinlichkeit auftreten könnten.

Das Abdomen wurde hinter dem sechsten Segment mit einer Präparationsschere durchtrennt und der verbleibende Teil vom Abdomen und Thorax anschließend durch zwei laterale Schnitte oberhalb der Pleuralleiste halbiert. Dabei war darauf zu achten, dass die lateral an beiden Seiten des Abdomens und oberhalb der Pleuralleisten befindlichen Stigmata intakt belassen wurden, um den Gasaustausch so wenig wie möglich zu beeinträchtigen. Am Thorax wurden die lateralen Schnitte etwas näher zur Dorsalseite hin geführt, damit die Muskeln M96 (dritter Abduktor der Coxa, nach SNODGRASS, 1929) nicht beschädigt wurden, deren Ansatzstellen lateral etwas höher an der Kutikula ansetzen. Nachdem auch die gesamte Dorsoventralmuskulatur mit der Präparationsschere auf dieser Höhe durchtrennt worden war, 
erfolgte dorsal ein Schnitt dicht hinter dem Kopf, wodurch die dorsale Hälfte dieses Thorax-/Abdomen-Präparates freigelegt und entfernt werden konnte. $\mathrm{Zu}$ diesem Zeitpunkt wurden erstmals einige Tropfen Ringer-Lösung (CLEMENTS and MAY 1974) auf den eröffneten Teil des Thorax gegeben.

Das Präparat musste nun zur weiteren Verwendung mit der Ventralseite nach unten auf einer flachen Metallschiene fixiert werden, die anterior abgeflacht war und posterior in einen rundgeformten Metallstab überging. Das vordere abgeflachte Ende hatte eine speziell angepasste Fläche, die lediglich das Sternum bedeckte (Abb. 3, D2D). Alle Extremitäten bis auf die bereits abgetrennten Hinterbeine blieben stets frei beweglich. Die Metallhalterung war mit California Red Wachs (Dentalwachs, Hersteller unbekannt) beschichtet, um eine spätere Fixierung des Präparates mit Minutiennadeln zu ermöglichen. Auf ihr waren senkrecht auch vier Spitzen von umgeknickten Minutiennadeln stabil in einem vorher ausgemessenen und an die durchschnittlichen Dimensionen eines Locusta-Thorax angepassten Abstand zueinander verlötet. Das Präparat wurde so mit dem Sternum auf diese Halterung aufgesetzt, dass die Spitzen beim Durchstechen lateral zu beiden Innenseiten der unteren Thoraxhälfte (nahe der Thoraxwand) positioniert waren. Stets war dabei darauf zu achten, dass das zentrale Nervensystem oder wichtige Teile der Beinmuskulatur nicht beschädigt wurden. Die Metallspitzen dienten zur stabilen Konterhalterung und Verankerung des Präparates. Einmal in die richtige Position gebracht, wurden weitere Minutiennadeln in leichter Schrägstellung zu beiden inneren Seiten nahe der Thoraxwand eingelassen, im Red Wachs verankert und das Präparat somit festgelegt. Zur vollständigen Fixierung des Thorax wurde das am Sternum vorher aufgetragene Wachs lateral mit einem Lötkolben aufgeschmolzen, so dass sich möglichst ein wasserdichter Verschluss zwischen dem Sternum des Präparates und dem California Red Wachs bilden konnte. Dies war auch deswegen wichtig, weil so einem späteren Auslaufen von Ringer-Lösung aus dem Präparat vorgebeugt werden konnte. Es war darauf zu achten, dass alle Extremitäten (beide Vorder- und Mittelbeine) weiterhin frei beweglich blieben. Der am Präparat belassene Kopf wurde über seitliche Wachsbrücken (Abb. 1, B; Heißklebewachs Pattex hot, Henkel) am Thorax fixiert. So konnte verhindert werden, dass sich etwaige durch Bewegung des Kopfes erzeugten Kräfte negativ auf die Stabilität der intrazellulären Ableitung auswirkten.

Nach der Fixierung des Präparates musste der Verdauungstrakt entfernt werden. Um 
ein Auslaufen von Verdauungssubstanzen in die Ringer-Lösung zu vermeiden, wurde der Darm zuvor dicht hinter dem Kopf mit einem Faden abgebunden, verknotet und zuletzt dicht dahinter mit der Präparationsschere durchtrennt und entfernt. Der gesamte Innenbereich von Thorax und Abdomen wurde nun mehrfach mit Ringer-Lösung gespült und anschließend permanent immer mit genug Lösung benetzt gehalten. Ein Wechsel der Ringer-Lösung erfolgte über die gesamte Dauer des Experiments in Abständen von etwa 10-15min.

Im nächsten Schritt wurde die Dorsoventral- und Flugmuskulatur großteils heraus präpariert, Speicheldrüsen, Fett- und weiteres Gewebe entfernt bis die thorakalen Ganglien (Meso- und Metathorakalganglion) freigelegt und gut zugänglich waren (Abb. 1, B). Die Muskeln M67, M87 und M 88 wurden nahe ihrer Ansatzstellen an der prothorakalen sternalen Spina (1Spn, nach SNODGRASS 1929) durchtrennt, die Muskeln M93 und M117 etwas weiter lateral. Mit einer groben Pinzette wurde die zwischen Meso- und Metathorakalganglion befindliche mesothorakale Apophyse (zweite Spina 2Spn, nach SNODGRASS 1929) knapp oberhalb der Konnektive parallel zu diesen ergriffen. So eingeklemmt konnte diese kutikuläre Struktur anschließend durch eine leichte Scherbewegung abgebrochen und zusammen mit den noch an ihr befindlichen Teilen der Muskulatur (M88, M93 und M117) entfernt werden. Dies war nötig, damit später eine speziell angepasste Metallhalterung unter dem Mesothorakalganglion positioniert werden konnte, um das Ganglion für die intrazelluläre Ableitung zu stabilisieren und zu fixieren. Auch die Längsmuskeln M60 wurden durchtrennt, damit sich eventuell auftretende Kräfte durch Bewegungen der Vorderbeine (beweglicher Prothorax) später nicht mechanisch auf das auf einer Metallplattform gelagerte Mesothorakalganglion übertragen konnten.

Mit einer feinen an der Spitze umgebogenen Minutiennadel wurde die Ganglionhülle (Neurolemm) entlang der Mittellinie für den Bereich der zu untersuchenden dorsalen Kommissuren lokal eröffnet. Gegebenenfalls wurde die Ganglionhülle zur besseren Sichtbarkeit manchmal mit Coomassie Blue (Brilliant Blue R, Sigma 0149, in RingerLösung) vorher leicht angefärbt. Das Ganglion war damit für intrazelluläre Ableitungen mit der Glasmikroelektrode im Bereich der dorsalen Kommissuren DClDCVI vorbereitet. 


\section{$2.4 \quad$ Versuchsaufbau}

Der gesamte Versuchsaufbau war in einen Faradayschen Käfig integriert, zur geeigneten Abschirmung der Myogramm- und intrazellulären Ableitungen gegen störende elektromagnetische Felder. Alle metallischen Geräte innerhalb des Faradayschen Käfigs wie auch alle verwendeten externen Elektrogeräte wurden über eine große Metallplatte innerhalb des Käfigs geerdet.

Nach allen vorbereitenden präparativen Schritten wurde der Stab der Metallschiene zusammen mit dem daran fixierten Präparat in den Schlitten eines Mikromanipulators (Bachofer, Typ 1.1 M) eingespannt. Dieser wiederum war über Stativmaterial an einem Magnetfuß (MG1 in Abb. 3, A) befestigt. So konnte das Präparat über der Laufkugelkonstruktion geeignet positioniert werden (Abb. 1, A-B und Abb. 3, A, D1). Alle nun folgenden Schritte erfolgten auch unter optischer Kontrolle mittels eines auf das Präparat ausgerichteten Binokulars (Leica, Wild M3C). Zur Beleuchtung diente die Kaltlichtquelle einer Schwanenhalslampe (Hund, Faserlichtquelle FLQ 85E, 85 Watt). Diese war zur genaueren Positionierung über dem Präparat über Stativmaterial an einem Magnetfuß (MG2 in Abb. 3, A; Bachofer, Modell nicht bekannt) befestigt. Zunächst wurde eine Metallplattform zur Stabilisierung und Halterung des Mesothorakalganglions mittels eines Mikromanipulators (Bachofer, Typ 3.1.7) in Position gebracht (anterior und in unmittelbare Nähe zum Ganglion). Die Plattform („Löffel“) wurde aus einem stabilen Draht (Stärke 1mm) gefertigt, an der Spitze abgeflacht und auf Ganglionmaß zugefeilt. Zur zusätzlichen Stabilisierung wurde mit einem 2-Komponenten-Kleber eine dickere Schicht auf den Löffel aufgetragen und nach Aushärten mit Aussparungen für das Ganglion und alle abgehenden Nerven und Konnektive (Abb. 3, D2 und D2D) geeignet zugefräst. Die Metallhalterung zur Stabilisierung des Mesothorakalganglions wurde gleichzeitig als Erdung (Bad-Referenz-Elektrode) sowohl für die intrazelluläre wie auch für die elektromyografischen Ableitungen benutzt. Der Anschluss der Myogrammelektroden wie auch der Bad-Elektrode zur Erdung erfolgte an einem externen Anschlussmodul des Muskelverstärkers (Anfertigung der institutseigenen Elektronik-Werkstatt, Verstärkung: 1000x) innerhalb des Faradayschen Käfigs (Abb. 3, A). Über eine weitere externe Anschlussbox des Intrazellulärverstärkers (NPI, SEC-05L) innerhalb des Käfigs wurde das elektrische Signal der Glasmikroelektrode aufgenommen und verstärkt. Die exakte Positionierung der Intrazellulärelektrode erfolgte unter Zuhilfe- 

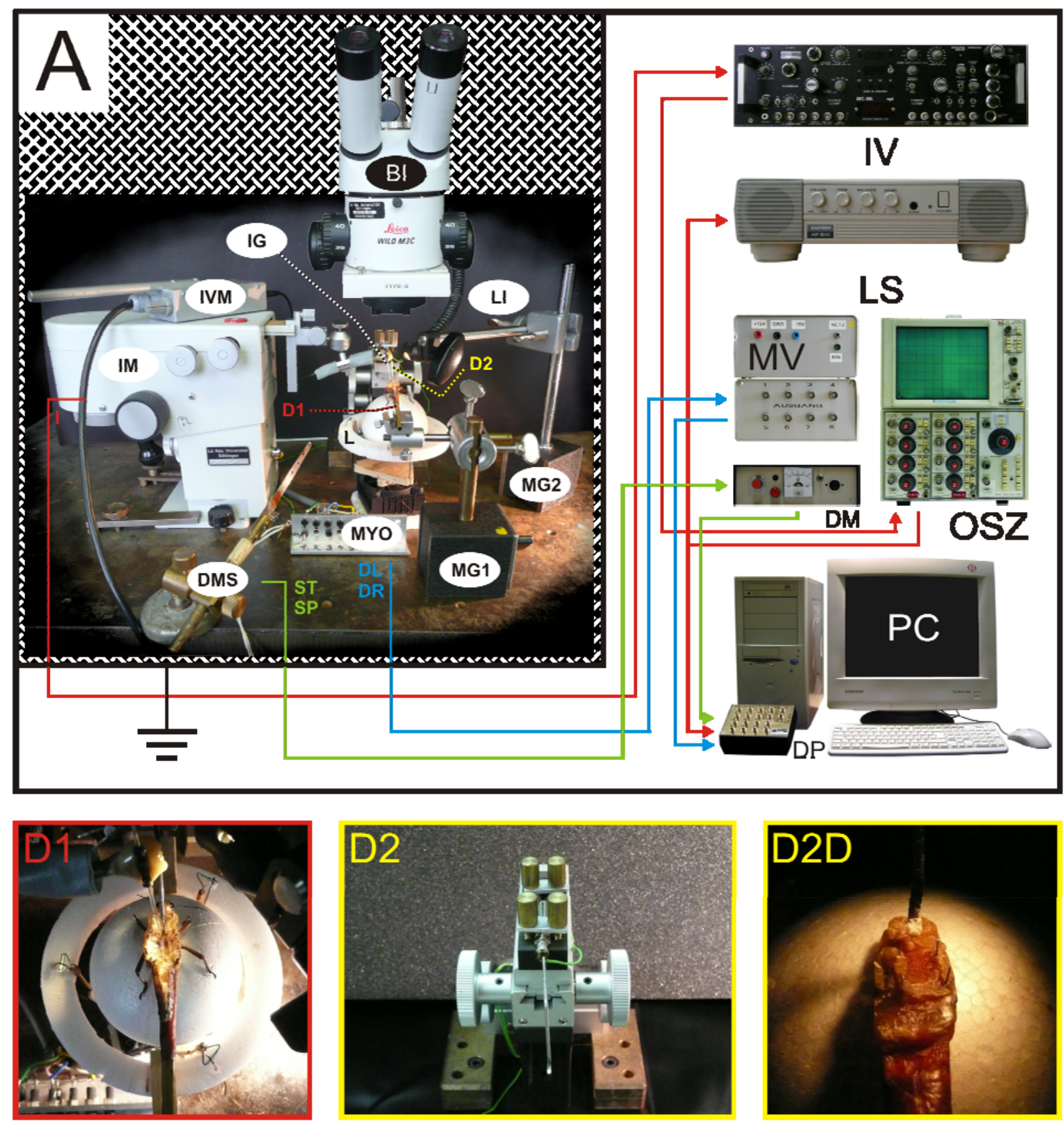

Abb. 3: Versuchsanordnung zur intrazellulären Ableitung von Interneuronen des Mesothorakalganglions bei Locusta migratoria. A: Übersicht zum lokalen Aufbau unter dem Faradayschen Käfig (Kasten mit Geflechtstruktur) und elektronische Peripheriegeräte. Die Detailausschnitte D1-D2 sind unterhalb vergrößert gezeigt. D1: Aufsicht auf das ventral gehalterte Präparat, Vorder- und Mittelbeine sind beweglich auf einer Laufkugel. Für Details zur Position der Glasmikroelektrode und der Ganglionstabilisierung siehe Abb. 1. D2: Bachofer-Manipulator zur Positionierung der Ganglionhalterung (Löffel). D2D: Detailausschnitt des Löffels mit Aussparungen zur Aufnahme des Ganglions und der ventralen Halterung des Präparates mit vier senkrecht stehenden Minutiennadeln.

Abkürzungen: BI: Binokular; DL,DR: Muskelableitungen vom Depressor trochanteris (M103a) vom rechten (DR) und linken (DL) Mittelbein; DM: Dehnungsmessstreifen, Verstärker; DMS: Dehnungsmessstreifen, Reizindikation; DP: DATAPAC-Anschlussmodul zur Datenaufnahme; I: Intrazelluläre Ableitung; IG: Glasmikroelektrode; IM: Manipulator für Glasmikroelektrode; IV: Intrazellulärverstärker; IVM: Externes Anschlussmodul, Intrazellulärverstärker; L: Laufkugel (Siehe Abb. 1); LI: Faserlichtquelle; LS: Lautsprecher; MG1: Magnetfuß und Stativmaterial zur Positionierung der Präparathalterung. MG2: Vorrichtung zur Positionierung der Lichtquelle (LI); MV: Muskelverstärker; MYO: Anschluss-Interface für Muskelableitungen; OSZ: Oszilloskop; PC: Computer zur Datenaufnahme; ST, SP: Reizindikation für taktile Tarsusreize (ST) und passives Auslenken (SP: Dauerpuls). 
nahme eines Mikromanipulators (Leitz). Zur Kennzeichnung und Unterscheidung der gegebenen taktilen Reize an einem der beiden Mittelbeintarsen wurde ein Dehnungmessstreifen benutzt (Anfertigung der institutseigenen Elektronik-Werkstatt, bipolares elektrisches Signal, abhängig von Auslenkrichtung und Beugungsstärke eines Metallstreifens). Dessen Signal wurde mit einem Produkt der Firma Shinohara Electrical Instruments Works LTD. verstärkt. Das vom Intrazellulärverstärker ausgegebene Signal wurde zur optischen Kontrolle zunächst zu einem Oszilloskop (Tektronix, TDS 210, digitales 2-Kanal Oszilloskop) geleitet und von dort einerseits zu einem Lautsprecher geführt (Eastern, HF-B40, zur zusätzlichen akustischen Kontrolle), andererseits über ein externes Anschlussmodul des verwendeten Systems zur Datenerfassung (Hard- und Software: Run Technologies, Datapac 2K2) mit einem Computer verbunden (AMD Athlon CPU: $1 \mathrm{GHz}$, DDR-SDRAM: $1 \mathrm{GHz}$, 266MHz, Grafikkarte: GeForce2 MX/MX400, Windows 2000 Professional). Die vom Muskelverstärker ausgegebenen elektrischen Signale wurden ebenso wie die vom Verstärker des Dehnungsmessstreifens kommenden Signale direkt an das DatapacModul angeschlossen.

\subsection{Versuchsdurchführung}

2.5.1 Intrazelluläre Ableitung, Reizung und Datenaufnahme

Die Glaselektroden (World Precision Instruments, Inc., 1,0mm Außendurchmesser, aus Borosilikat und mit Filament) wurden mit einem Vertikal-Puller (David Kopf Instruments, Model 700C) gezogen, mit einer 5-\%igen Lucifer Yellow-Lösung (Sigma L-0259) in der Spitze und dann mit einer Lithium-Chlorid-Lösung (1M LiCl) aufgefüllt. Im Experiment wurden nur Elektroden verwendet, die vor dem Einstechen in das Ganglion einen Widerstand zwischen 80-200M $\Omega$ hatten. Um eine genauere Positionierung der Elektrode im Zielgebiet (eine der dorsalen Kommissuren) zu ermöglichen, wurde diese mit Hilfe des Mikromanipulators in nahezu vertikaler Orientierung über der Ableitstelle platziert, dann mittels des Feintriebes soweit vorsichtig angenähert, bis der erste Kontakt erfolgte, und anschließend durch abwechselndes leichtes Klopfen auf den Intrazellulärmanipulator und Bedienung dessen Feintriebes weiter bis in die Region der betreffenden dorsalen Kommissur 
herabgesenkt. Bei jeder erfolgreichen Penetration einer Zelle wurden mit einem feinen und weichen Pinsel taktile Reize jeweils an der Dorsalseite einer der Tarsen beider Mittel- und Vorderbeine sowie auch an Haaren durchgeführt, die lateral am Thorax oder Abdomen lokalisiert sind. Im Falle einer interessanten Veränderung der neuronalen Aktivität im Kontext der Reizungen und ausgelösten bilateralen oder intersegmental gerichteten Reflexe wurde die kontinuierliche Aufzeichnung der Neuronaktivität zusammen mit der Muskelaktivität über die Datapac-Software gestartet (Abtastrate: $7-10 \mathrm{kHz}$ ).

Im Folgenden wurden zunächst taktile Einzelreize an der Dorsalseite jeweils einer der beiden Mittelbeintarsen gegeben bei gleichzeitiger entsprechender Auslenkung des Dehnungsmessstreifens zur Reizindikation, die ebenfalls am Computer aufgezeichnet wurde. In den Abbildungen zur Physiologie ist die Reizindikation dabei als „ST“ gekennzeichnet (Stimulus: taktil). Bei einer Reizung am Tarsus des rechten Mittelbeins wurde der Dehnungsmessstreifen manuell nach links ausgelenkt. Dies entspricht ursprünglich einem negativen Ausschlag des aufgezeichneten Signals der Reizindikation. Bei einer Reizung links wurde der Streifen nach rechts ausgelenkt, was zu einem positiven Ausschlag in der Reizindikation führt. In den hier gezeigten Abbildungen zur Physiologie wurde in der Darstellung der Reizindikation auf eine solche Unterscheidung verzichtet, da die jeweilige Art der durchgeführten Reizungen in den Beschriftungen der Abbildungen erklärt wird. In der betreffenden Spur werden in diesem Fall normierte Einheitspulse mit positiver Flanke für taktile Tarsusreizung angegeben.

Bei einigen Präparaten ließen sich bilaterale (und/oder intersegmentale) Reflexe manchmal kaum sichtbar auslösen. In diesen Fällen erfolgte nur eine einmalige Reizindikation zu Beginn einer kontinuierlichen taktilen Reizung des betreffenden Tarsus bis zum Reizerfolg (Beinbewegungen, sichtbar ausgelöster Reflex). Daher treten in den zeitlich korrelierten Abbildungen der physiologischen Daten entsprechend Verzögerungen zwischen Reizbeginn (Standartpuls) und Reizerfolg (Auftreten von gemessener Muskelaktivität) auf. Aus diesem Grund wurde zusätzlich zum normierten Einheitspuls (der den Beginn der kontinuierlichen taktilen Reizung markiert) auch noch eine gestrichelte Linie eingetragen, die den Zeitraum kennzeichnet, in welchem kontinuierlich gereizt wurde.

Für den Fall, dass sich Reflexe auch durch kontinuierliche taktile Reizung kaum noch erkennbar auslösen ließen, wurden beide Mittelbeine nacheinander auch passiv 
vorsichtig angehoben, indem die jeweilige Tibia mit der Pinselspitze nach dorsal ausgelenkt wurde. Die Möglichkeit einer Beeinträchtigung der neuronalen Aktivität durch eine mechanische Veränderung der Ableitbedingungen (Ableitartefakt) wird an gegebener Stelle berücksichtigt. Die Reizindikation solcher passiven Auslenkbewegungen erfolgte durch Dauerauslenkung des Dehnungsmessstreifens in der entsprechenden Richtung für die Zeit des Anhebens der Tibia. Zur Darstellung solcher passiven Auslenkbewegungen der Mittelbeine in der Reizindikation (Kennzeichnung: SP, für Stimulus: passive Auslenkung) wurden zeitlich zur Dauer der Auslenkung korrelierte Rechteckpulse mit positiver Flanke benutzt (durchgängige, keine gestrichelte Linie).

Nachdem alle taktilen Tarsusreize an beiden Mittelbeinen oder gegebenenfalls deren passives Anheben durchgeführt worden war, wurde die Aufzeichnung angehalten und der entsprechende Endzeitpunkt notiert, um in der Spur zu einem späteren Zeitpunkt Reize an Mittelbeinen von Reizungen an den Tarsen der Vorderbeine oder von lateralen Haaren unterscheiden zu können. Daraufhin wurde die Aufzeichnung fortgesetzt und ebensolche Reizungen (zur Auslösung intersegmental gerichteter Reflexe) durchgeführt (keine differenzierte Reizindikation in der Aufzeichnung). Die Aufnahme wurde danach wieder unterbrochen, die Zeit und ebenso die Art der neuronalen Reaktion in Abhängigkeit zur jeweiligen Reizung notiert. Wenn die intrazelluläre Ableitung zu diesem Zeitpunkt noch stabil war, wurde die Datenregistrierung ein weiteres Mal fortgesetzt und überprüft, inwieweit Hyper- oder Depolarisieren des Membranpotentials der abgeleiteten Zelle die zu erwartenden Effekte auf das neuronale Verhalten hatten. Auch konnte durch die simultane Aufzeichnung der neuronalen und der Muskelaktivitäten überprüft werden, ob die durch Depolarisieren erhöhte neuronale Aktivität auch einen direkten Einfluss auf die Muskelaktivität hatte (sichtbares Einsetzen von Beinbewegungen und Auftreten von Muskelaktivitäten in der synchron am Computermonitor dargestellten Aufzeichnung). 
2.5.2 Intrazelluläre Färbungen mit Lucifer Yellow, histologische Aufarbeitung für die Fluoreszenz-Mikroskopie und zweidimensionale Rekonstruktion der neuronalen Morphologie durch Zeichnung

Nach der Aufzeichnung der physiologischen Eigenschaften einer intrazellulär stabil abgeleiteten Zelle wurde diese durch Anlegen eines hyperpolarisierenden Stromes (5-15min bei 7-10nA, abhängig von der Stabilität der Ableitung) iontophoretisch gefärbt. Danach wurden die Konnektive oberhalb des Pro- und unterhalb des Metathorakalganglions durchtrennt und dementsprechend alle drei Ganglien zusammenhängend herauspräpariert und in eine mit Sylgard beschichtete Glasschale überführt (Dorsalseite nach oben). Der entsprechende Teil des Nervensystems wurde mit U-förmig gebogenen Minutiennadeln am Sylgard fixiert und mit Ringer-Lösung benetzt. Danach wurden mit einer feinen Pinzette oder mit einer feinen Pinzetten-Schere (John Weiss \& Sohn, LTD. London) störende Gewebeteile wie Fett- und Nährgewebe oder Tracheenreste entfernt. Anschließend, jedoch nicht vor Ablaufen einer Diffusionszeit des Lucifer Yellow für mindestens 30 Minuten, konnten die Ganglien in ein Blockschälchen überführt und für weitere 30 Minuten in einer 4\%igen Paraformaldehyd-Lösung fixiert werden. Nach Entwässerung des Gewebes in einer aufsteigenden Alkoholreihe (je 10min in 30\%, $40 \%, 50 \%, 70 \%, 80 \%, 90 \%$ und 2x für 15 Minuten in 99\% Ethanol) wurde das Präparat mit der Dorsalseite nach oben auf einem Objektträger ausgebreitet, mit Methylsalicylat zur Aufklarung beschichtet und mit einem Deckgläschen abgedeckt. Nach einer Einwirkzeit von wenigstens 20 Minuten erfolgte die Analyse der Struktur des Neurons unter dem Fluoreszenz-Mikroskop (Leitz, Aristoplan). Die Morphologie des Neurons wurde sukzessive Bild für Bild mit einer Digitalkamera (Nikon, Coolpix 950) über den gesamten Bereich von fluoreszierenden neuronalen Verzweigungen (im Mesothorakalganglion und - wenn vorhanden - auch im Pro- und Metathorakalganglion) von dorsal nach ventral fotografiert. Dabei wurden Objektive mit zehn- oder fünfundzwanzigfacher Vergrößerung benutzt. $\mathrm{Da}$ es sich bei den meisten kommissuralen Neuronen auch um große intersegmentale Interneurone handelte, wurde die Fokalebene nicht in standardisierten Schritten verändert, sondern derart, dass jeweils immer nächstfolgende Abschnitte fokal abgebildet waren (Abstände zwischen den Fokalebenen abhängig von der Komplexität der Verzweigungen in diesem Bereich). Zur späteren Bestimmung der Dimensionen wurde ein 
Objektmikrometer (Leitz) für jede der benutzen Vergrößerungen bei den jeweils aktuellen Einstellungen fotografiert. Die digitalen Bilder (TIFF-Format, Auflösung 1600x1200 Pixel) wurden über ein entsprechendes Lesegerät (Pixo Media, USB-CFII) auf den Auswertungscomputer übertragen. Mithilfe der Corel Draw-Software (Corel Graphics Suite, Version 12) erfolgte eine zweidimensionale Rekonstruktion der Morphologie des Neurons durch Zeichnung. Dazu wurden die benötigten Bildsequenzen in das Programm geladen und in der richtigen Reihenfolge übereinander gelegt (Bildstapel). Gezeichnet wurden jeweils alle fokal in einem Bild abgebildeten neuronalen Strukturen und dies entsprechend sukzessive Bild für Bild (von dorsal nach ventral). Die optische Kontrolle des Verlaufs von neuronalen Verzweigungen erfolgte unter Benutzung eines Bildmanager-Programms (ACD See Software, Version 7). In der Zeichnung wurden die am weitesten dorsal liegenden Strukturen durchgehend, eventuell weiter ventral und darunter verlaufende Fasern mit Unterbrechung gezeichnet. Aufgrund der Komplexität der Verzweigungen bei einigen Interneuronen wurde zur besseren Zuordnung zusammenhängenden Verzweigungen jeweils eine bestimmte Zeichnungsfarbe zugeordnet. Das Augenmerk liegt hier jedoch lediglich auf der besseren Unterscheidbarkeit von benachbarten Verzweigungen. In einer Zeichnung können gegebenenfalls mehrere Verzweigungen die gleiche Farbe haben, wenn sie weit genug auseinander liegen, so dass eine Unterscheidung immer noch leicht möglich ist.

\subsubsection{Auswertung der physiologischen Daten}

Die Analyse der neuronalen Reaktionen erfolgte jeweils separat für taktile Reizung an den beiden Mittelbeintarsen, für passives Anheben der Mittelbeine (falls durchgeführt) und für aktive Bewegungen (durch Einzelreiz ausgelöste BewegungsSequenz oder spontane Bewegungen ohne Reizung). Die gewählten Zeitauflösungen der zu analysierenden Ausschnitte (Sektionen) richtete sich nach dem aufgetretenen neuronalen Verhalten und ihrer Korrelation zu den simultan gemessenen Muskelaktivitäten sowie einer gelegentlich aufgetretenen Verzögerung von der Reizung bis zum sichtbar ausgelösten Reflex (gemessene erhöhte Muskelaktivität). Für die jeweiligen Abschnitte wurden mittels der Datapac-Software (Run Technologies, Datapac 2K2) die Intervalle zwischen allen benachbarten Spikes 
(„Onset-to-Onset“ -Intervall: „Onset" eines als „Event“ markierten Spikes bis zum "Onset" des nächsten Spikes) innerhalb des gewählten Zeitausschnittes berechnet (Einheit: [ms]). Diese Daten wurden zusammen mit den absoluten Zeitwerten („Onset-Time“, Zeitpunkt t(n) des Auftretens eines Spikes (Events) „n“ in der OriginalAufzeichnung; ansteigende Flanke; Einheit: [ms]) zur weiteren Bearbeitung exportiert (als *.txt-Datei). Screenshots von den Originalaufzeichnungen (je eine Spur für die intrazelluläre Ableitung IN, die Muskel-Ableitungen DR und DL und die Reizindikation ST oder SP) wurden für die jeweils korrespondierenden Zeitausschnitte gewählt und im *.emf-Format (Enhanced Metafile) abgespeichert. Die exportierten Rohdaten („Onset-to-Onset“ -Intervalle und Zeitwerte $t(n)$ ) wurden in MS Excel (MS Office 2000 Professional) importiert. Die absoluten Zeitwerte $t(n)$ wurden dann auf die Zeitskala der korrespondierenden Ausschnitte der Physiologie-Daten (dargestellte Sektionen der Originalspuren) normiert. Das bedeutet, dass dem Beginn eines jeden Ausschnitts der Zeitwert Null zugeordnet wurde und die absoluten Zeitwerte $t(n)$ auf diesen Bezug umgerechnet wurden (Zeitwerte $t\left(n^{*}\right)$ ).

In den hier gezeigten Abbildungen zur Physiologie ist der Verlauf der neuronalen Aktivität durch eine gemittelte Instantanfrequenz angegeben. Zu ihrer Berechnung wurden in Excel jeweils zwei benachbarte "Onset-to-Onset" -Intervalle gemittelt (gleitendes Mittel). Gemäß der Definition der Instantanfrequenz wird jede berechnete Frequenz einem Zeitpunkt $t(i)$ zugeordnet, der zeitlich genau in der Mitte zwischen zwei benachbarten Spikes liegt, aus deren „Onset-to-Onset“ -Intervall auch der jeweilige Frequenzwert zu berechnen ist $\left(t(i)=t\left(n^{*}\right)+\left(\left(t\left(n^{*}+1\right)-t\left(n^{*}\right)\right) / 2\right)\right)$. Wegen der durchgeführten Mittelung (gleitendes Mittel) von jeweils zwei benachbarten "Onset-to-Onset" -Intervallen mussten die gemittelten Intervalle wiederum entsprechend einem Zeitpunkt $t\left(i^{*}\right)$ zugeordnet werden, der sich durch Mittelung von jeweils zwei benachbarten $\mathrm{t}(\mathrm{i})$-Werten berechnet $\left(\mathrm{t}\left(\mathrm{i}^{\star}\right)=\mathrm{t}((\mathrm{i})+((\mathrm{t}(\mathrm{i}+1)-\mathrm{t}(\mathrm{i})) / 2))\right.$. Zur Berechnung der Instantanfrequenz aus den gemittelten „Onset-to-Onset" -Intervallen wurden letztere (in der Einheit [ms]) zunächst gerundet auf eine Nachkommastelle (die Aufnahme der physiologischen Daten erfolgte bei einem Sample-Intervall von $100 \mu$ s oder $150 \mu \mathrm{s}$ ) und dann in die Einheit [s] umgerechnet (4 Nachkommastellen, keine weitere Rundung). Durch einfache Kehrwertbildung wurden dann schließlich die Werte der Instantanfrequenz berechnet. Zur Berechnung der jeweils zu einer dargestellten Sektion korrespondierenden Spontanfrequenz wurde zunächst in der Originalspur der Neuronaktivität nach einem Zeitabschnitt gesucht, der unmittelbar 
vor dem jeweils betrachteten Reiz-Reaktions-Verlauf lokalisiert war, innerhalb dessen keine Reizung stattgefunden hat und in dessen Verlauf das Neuron bei einem möglichst gleichbleibenden Tonus aktiv war. Die Zeitfenster wurden dabei möglichst groß gewählt, nach Möglichkeit bei einer Dauer von wenigstens vier Sekunden. In wenigen ungünstigen Fällen (unregelmäßiger Tonus der Neuronaktivität beziehungsweise zwei oder mehrere kurz hintereinander folgende Einzelreize) wurde entweder das Zeitfenster angepasst (Verkürzung des Zeitfensters auf minimal 4s) oder zeitlich so weit wie nötig nach vorne verlagert (innerhalb eines Bereiches ohne jegliche Reizung). Es wurden wieder mittels der Datapac-Software die Spike-Intervalle in der Einheit [ms] berechnet („Onset-to-Onset“ -Intervalle) und die als *.txt-Datei entsprechend exportierten Werte in MS Excel importiert. In Excel wurden diese Intervalle (Einheit [ms]) zunächst wieder auf eine Nachkommastelle gerundet und dann der Mittelwert über alle Intervalle berechnet. Umrechnung des mittleren Intervalls in die Einheit [s] (vier Nachkommastellen, keine Rundung) und einfache Kehrwertbildung ergaben den Wert der mit der jeweiligen Sektion korrespondierenden Spontanfrequenz.

Alle in MS Excel berechneten relevanten Daten (Werte der Instantanfrequenz, normierte korrespondierende Zeitwerte $\mathrm{t}\left(\mathrm{i}^{*}\right)$ und Wert der zugehörigen Spontanfrequenz) wurden im Programm „Sigma Plot“ (Systat Software, Inc.; Version 10) importiert, grafisch dargestellt und als *.wmf-Datei exportiert. Die hier in den Physiologieteilen gezeigten Abbildungen (zeitlich synchronisierte Darstellung von Physiologiedaten und Instantan-/Spontanfrequenzen) wurden unter Benutzung der Corel Draw Software (Corel Graphics Suite, Version 12) erstellt. Die als *.wmfDateien importierten Grafiken der Instantan- und Spontanfrequenzen konnten in diesem Programm als Vektorgrafiken mit den ebenfalls importierten Sektionen der Physiologiedaten (*.emf-Dateien) arrangiert werden.

In jeder Abbildung zur Physiologie des jeweiligen Neurons befindet sich - in zeitlicher Synchronisation - in der obersten Spur der Ausschnitt der intrazellulären Ableitung des Neurons (IN), darunter die korrespondierenden Abschnitte der elektromyografischen Aufzeichnungen (DR: Depressor trochanteris, rechtes Mittelbein; DL: Depressor trochanteris, linkes Mittelbein), die Spur der Reizindikation (ST: Taktiler Stimulus, an der Dorsalseite des betreffenden Mittelbein-Tarsus; SP: Passives Anheben vom betreffenden Mittelbein) und die Grafik der Instantan- und Spontanfrequenz der neuronalen Aktivität. Bei letzterer ist die Frequenz unter 
Angabe vom Minimal- und Maximalwert des Frequenzbereiches (Einheit [Hz]) auf der $y$-Achse aufgetragen, die Zeiteinheit ist auf der x-Achse abzulesen (normiert auf eine Zeitauflösung von einer Sekunde).

\subsection{Nomenklatur}

\subsubsection{Nomenklatur der beschrieben kommissuralen Interneurone}

Die Nomenklatur der nachfolgend beschriebenen Neurone ist derartig gewählt, dass der jeweiligen Typenbezeichnung CIN-I (commissural interneuron, bilateraler Verzweigungstyp I) oder CIN-II (medianer Verzweigungstyp II) in Klammern einzelne oder kombinierte Symbole angehängt werden, die indizieren sollen, ob das Axon des Neurons im Konnektiv aufsteigt (a: ascending), absteigt (d: descending), oder durchläuft (ad: ascending-descending). Weitere Symbole nach der Klammer geben an ob es sich um ein kontralateral zum Soma (c: contralateral) oder ipsilateral zum Soma (i: ipsilateral) gelegenes Axon handelt. Da dennoch mehrere Neurone mit bislang gleicher Nomenklatur vorkommen können, wird zur Unterscheidung von „1“ bis „n“ durchnummeriert.

Beispiel: CIN-I(ad)c1 ist das erste gefundene kommissurale Interneuron vom bilateralen Verzweigungstyp I, das ein im kontralateralen Konnektiv durchlaufendes Axon besitzt.

\subsubsection{Nomenklatur bekannter morphologischer Strukturen im Mesothorakal- ganglion}

Die intrazellulären Ableitungen aller hier dargestellten kommissuralen Interneurone erfolgten durch Einstechen der Ableitelektrode entlang der Mittellinie des Mesothorakalganglions sowie in der näheren Umgebung der dorsalen Kommissuren (dorsal commissures, DCI-DCVI). Deren Lokalisation erfolgte in Orientierung an die in der Arbeit von PFLÜGER et al. (1988) gezeigten Zeichnungen von Transversalschnitten eines mit Osmium und Ethyl-Gallat behandelten Mesothorakalganglions. Alle zur Beschreibung der Morphologie der hier dargestellten Neurone heran- 


\section{A Morphologische Strukturen im Mesothorakalganglion: Übersicht 1}
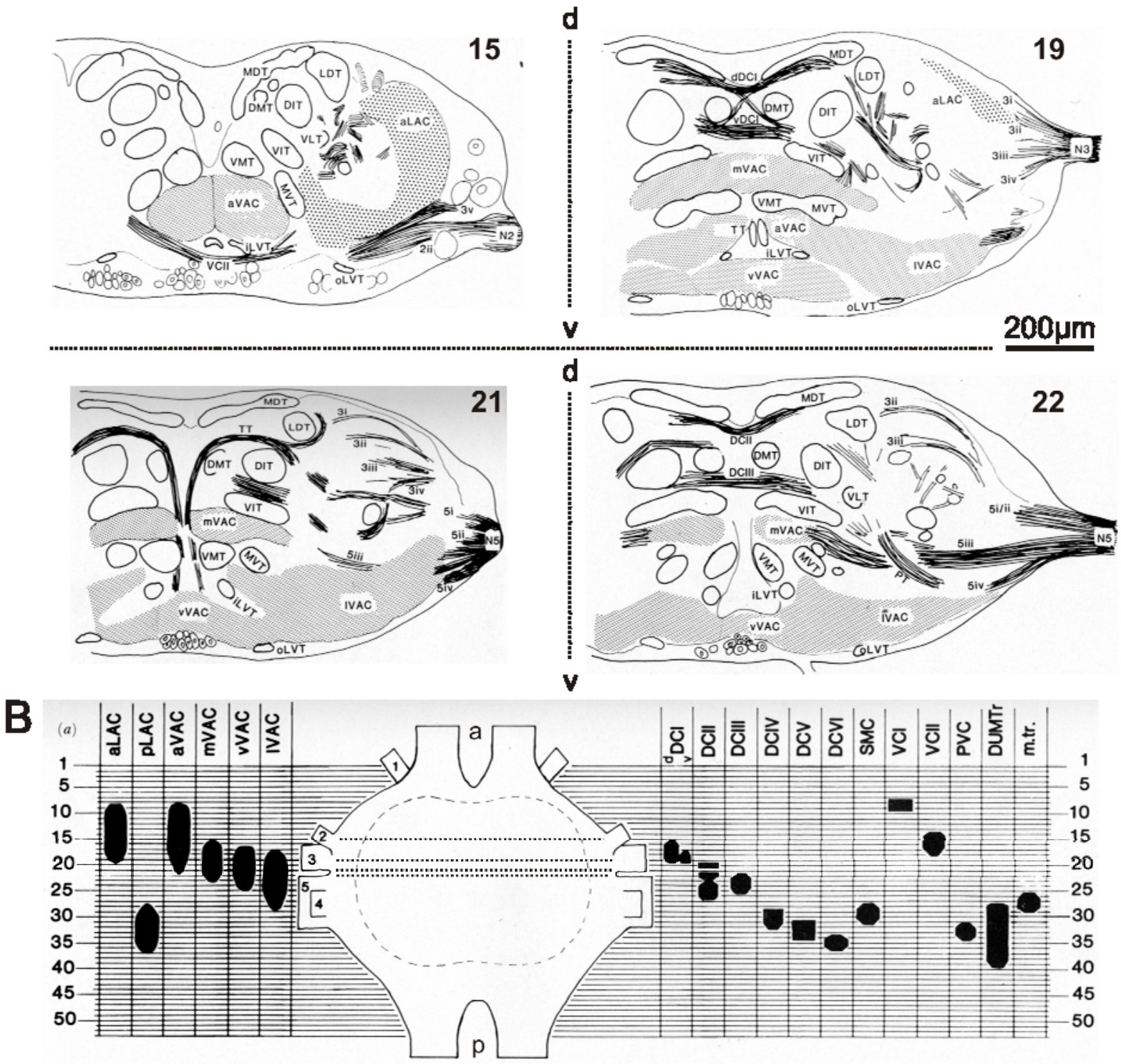

Abb. 4: Morphologische Strukturen im Mesothorakalganglion von Locusta migratoria, Übersicht 1. A-B: Zeichnungen entnommen aus: PFLÜGER et al. 1988; leicht modifiziert. Die Beschreibung der Abkürzungen erfolgt für alle in dieser Arbeit zitierten morphologischen Strukturen. A: Querschnittsektionen durch das Mesothorakalganglion, die die Lokalisation der Kommissuren, Trakte und Neuropile zeigen. Die jeweilige Zahl, die einer Sektion zugeordnet ist, verweist auf die in B gezeigte Schnittebene. B: Aufsicht auf die Ventralseite des Mesothorakalganglions und Vergleich mit der relativen Lage bekannter Kommissuren, Trakte und Neuropile. Die gestrichelten Linien sind mit dem Raster der Schnittebenen korreliert und verweisen auf die in A bezifferten Querschnittsektionen.

Abkürzungen: a: Anterior, aLAC: Anterior lateral association centre, aVAC: Anterior ventral association centre, d: dorsal, DCI-DCVI: Dorsal commissures I-VI, DIT: Dorsal intermediate tract, DMT: Dorsal median tract, LDT: Lateral dorsal tract, IVAC: Lateral ventral association centre, MDT: Median dorsal tract, mVAC: Median ventral association centre, MVT: Median ventral tract, N2: Nerv 2, N3: Nerv 3, N5: Nerv 5, p: Posterior, pLAC: Posterior lateral association centre, SMC: Supramedian commissure, TT: T-tract, v: Ventral, VIT: Ventral intermediate tract, VLT: Ventral lateral tract, VMT: Ventral median tract, vVAC: Ventralmost ventral association centre. Die hier erklärten Abkürzungen werden auch gesondert in der Liste der verwendeten Abkürzungen aufgeführt. 


\section{A Morphologische Strukturen im Mesothorakalganglion: Übersicht 2}
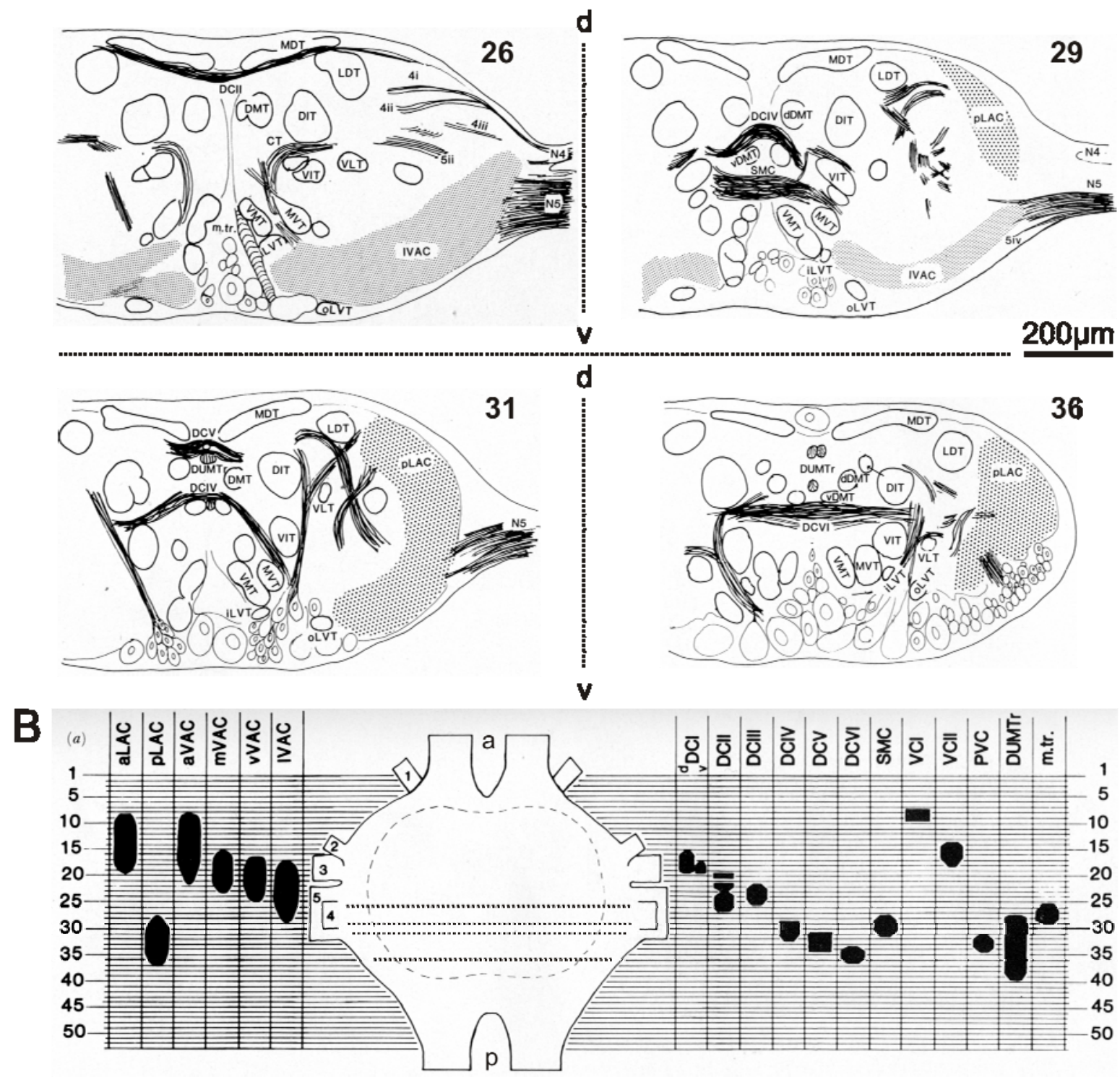

Abb. 5: Morphologische Strukturen im Mesothorakalganglion von Locusta migratoria, Übersicht 2. A-B: Zeichnungen entnommen aus: PFLÜGER et al. 1988; leicht modifiziert. Die Beschreibung der Abkürzungen erfolgt für alle in dieser Arbeit zitierten morphologischen Strukturen. A: Querschnittsektionen durch das Mesothorakalganglion, die die Lokalisation der Kommissuren, Trakte und Neuropile zeigen. Die jeweilige Zahl, die einer Sektion zugeordnet ist, verweist auf die in B gezeigte Schnittebene. B: Aufsicht auf die Ventralseite des Mesothorakalganglions und Vergleich mit der relativen Lage bekannter Kommissuren, Trakte und Neuropile. Die gestrichelten Linien sind mit dem Raster der Schnittebenen korreliert und verweisen auf die in A bezifferten Querschnittsektionen.

Abkürzungen: a: Anterior, aLAC: Anterior lateral association centre, aVAC: Anterior ventral association centre, d: dorsal, DCI-DCVI: Dorsal commissures I-VI, DIT: Dorsal intermediate tract, DMT: Dorsal median tract, LDT: Lateral dorsal tract, IVAC: Lateral ventral association centre, MDT: Median dorsal tract, mVAC: Median ventral association centre, MVT: Median ventral tract, N4: Nerv 4, N5: Nerv 5, p: Posterior, pLAC: Posterior lateral association centre, SMC: Supramedian commissure, v: Ventral, VIT: Ventral intermediate tract, VLT: Ventral lateral tract, VMT: Ventral median tract, VVAC: Ventralmost ventral association centre. Die hier erklärten Abkürzungen werden auch gesondert in der Liste der verwendeten Abkürzungen aufgeführt. 
gezogenen bekannten Referenzstrukturen innerhalb des Mesothorakalganglions sind in zwei Übersichten (Abb. 4-5) gezeigt, die ausgewählte Zeichnungen aus der Arbeit von PFLÜGER et. al. (1988) enthalten. Neben der jeweiligen dorsalen Kommissur, durch die der primäre Neurit des betreffenden Neurons verläuft, sind dies vor allem die bekannten Neuropilregionen wie beispielsweise das anteriore oder posteriore laterale Assoziationszentrum (aLAC, pLAC). Im Gegensatz zu den verschiedenen ventralen Assoziationszentren (aVAC, IVAC, vVAC und mVAC), in welche jeweils hauptsächlich nur ein bestimmter Mechanorezeptortyp projiziert und in welchen in der Regel keine Verzweigungen von Motoneuronen zu finden sind, projiziert eine Vielzahl von verschiedenen Mechanorezeptortypen in diese dorsal gelegenen lateralen Assoziationszentren (PFLÜGER et. al., 1988). Daneben verzweigen hier auch zahlreiche Motoneurone, so dass hier auch eine direkte Verschaltung von sensorischen Neuronen und von lokalen oder intersegmentalen Interneuronen auf Motoneurone erfolgen kann. Weitere Referenzstrukturen, die häufiger im Zusammenhang mit der Beschreibung der Morphologie eines in dieser Arbeit dargestellten Neurons genannt werden, sind die longitudinalen Trakte. In ihnen verlaufen sehr viele intersegmentale Fasern, die die Konnektive passieren. Da diese longitudinalen Trakte im Ganglion nicht direkt nebeneinander vorliegen, werden sie je nach ihrer Lokalisation unterschieden in den dorsal-medianen (DMT), den dorsalintermediären (DIT), den median-dorsalen (MDT) und den lateral-dorsalen Trakt (LDT). Weitere ventral gelegene Trakte sind der median-ventrale (MVT), der lateralventrale (LVT), der ventral-intermediäre (VIT), der ventral-laterale (VLT) und der ventral-mediane Trakt (VMT).

2.6.3 Erläuterungen zu einigen bei der Beschreibung von Morphologie und Physiologie der Neurone verwendeten Begrifflichkeiten

In den detaillierten Darstellungen der einzelnen hier vorgestellten Neurone erfolgt jeweils zuerst eine Beschreibung ihrer Morphologie und anschließend werden die physiologischen Daten erklärt. Da es sich bei den gezeigten Abbildungen zur Morphologie um zweidimensionale Rekonstruktionen (Zeichnungen) der Struktur des betreffenden Neurons im jeweiligen Ganglion handelt, werden die Termini „lateral“ und „median“, sowie „anterior“ und „posterior“ zum Verweis auf die Lokalisation einer 
neuronalen Struktur in dieser horizontalen Ebene verwendet. Um jedoch zusätzlich auch eine allgemeine Vorstellung davon zu geben, in welchem Bereich neuronale Verzweigungen bezüglich der Dorsoventral-Achse lokalisiert sind, werden im Ganglion bezüglich dieser Achse die drei Regionen „dorsal“, „intermediär“ und „ventral“ unterschieden. Für den Fall, dass eine neuronale Struktur über einen weiteren Bereich verzweigt, der genau zwischen zwei benachbarten Regionen liegt, werden entsprechende Termini kombiniert und durch Bindestrich getrennt: Einer neuronalen Verzweigungsstruktur, die dem primären Neuriten beispielsweise in der intermediären Region entspringt, die sich aber noch deutlich bis nach dorsal fortsetzt, wird entsprechend der morphologische Terminus „intermediär-dorsal“ zugeordnet. In entsprechender Weise werden auch alle anderen Übergangsbereiche beschrieben: Einem Soma, das beispielsweise ventral, anterior und lateral gelegen ist, werden die Termini „ventral“ und „anterior-lateral“ zugeordnet.

Aus Gründen der Einheitlichkeit beziehen sich die Begriffe „ipsilateral“ und „kontralateral“ sowohl in den Beschreibungen zur Morphologie der Neurone als auch in den Darstellungen zu ihrer Physiologie stets auf die Position des Somas.

Beispiele: Ein Neuron mit einem im kontralateralen (rechten) Konnektiv verlaufenden Axon hat ein im ipsilateralen (linken) Hemiganglion gelegenes Soma.

Ein kontralateraler taktiler Reiz an der Dorsalseite eines Tarsus betrifft das linke Mittelbein, wenn das in diesem Zusammenhang physiologisch beschriebene Neuron ein im ipsilateralen (rechten) Hemiganglion lokalisiertes Soma besitzt.

Soweit möglich werden bei der Beschreibung von neuronalen Verzweigungen auch die im Mesothorakalganglion bekannten morphologischen Strukturen berücksichtigt (Abb. 4-5; Kommissuren, Trakte und Neuropile; nach PFLÜGER et al. 1988). Da aber außer der Struktur des betreffenden Neurons selbst und den Dimensionen des Ganglions sonst nichts bekannt ist, können nur ungefähre Angaben gemacht werden, wenn eine bestimmte neuronale Struktur in der näheren Umgebung einer zur Beschreibung herangezogenen Referenzstruktur liegt: Wenn eine Verzweigung 
beispielsweise auf dorsal gelegenem Niveau bis weit nach posterior-lateral zieht, besteht die Möglichkeit, dass sie auch im posterioren lateralen Assoziationszentrum verzweigt. Aber dies ist für sich noch kein eindeutiges Argument.

Viele der hier beschriebenen Neurone haben charakteristische Verzweigungsbereiche, die von ihrer Lage her mit der Lokalisation anderer Strukturen des Mesothorakalganglions wie Nerven oder Konnektive in Relation gesetzt werden können. Dies wurde auch im Sinne einer differenzierteren Beschreibung von neuronalen Verzweigungsregionen versucht. Viele Neurone verzweigen häufig in einem Bereich, der in direkter Verlängerung zwischen den ipsilateralen sich longitudinal gegenüberliegenden Konnektiven liegt. Die durch die Konnektive ziehenden Fasern verteilen sich innerhalb des Ganglions auf verschiedene longitudinale Trakte (Abb. 4-5), die ihrerseits an unterschiedlichen Stellen durch das Ganglion verlaufen und mehr oder weniger weit voneinander entfernt sein können. Daher lassen sich größere Verzweigungsbereiche von ihrer Lokalisation her oft nicht eindeutig mit diesen Referenzstrukturen in Beziehung setzen. In solchen Fällen wurde stattdessen eine alternative Beschreibung wie beispielsweise „ein Bereich relativ zur Höhe der sich longitudinal gegenüber liegenden ipsilateralen Konnektive“ benutzt, da die Begrifflichkeit „auf Höhe der Konnektive“ missverständlich wäre. Viele Somata der gefundenen Neurone liegen (anterior oder posterior) häufig nahe des inneren oder äußeren Randes dieses gerade genannten Bereiches. Ihre Lokalisation wird an entsprechender Stelle im Text als „Bereich relativ zu einer gedachten Verbindungslinie durch den inneren (beziehungsweise äußeren) Randbereich der ipsilateralen sich longitudinal gegenüberliegenden Konnektive“ beschrieben. Liegen einzelne Verzweigungen zentral in diesem Bereich, wird dies an entsprechender Stelle durch die Beschreibung „relativ zu einer gedachten, zentral durch sie sich longitudinal gegenüberliegenden Konnektive verlaufenden Verbindungslinie“ zum Ausdruck gebracht. Entsprechend vergleichbare Umschreibungen finden auch Verwendung, wenn sich neuronale Verzweigungen mit den vom Ganglion abgehenden Nerven von ihrer Lage her in Relation gesetzt werden können. 
2.7 Anfertigung medianer Sagittalschnitte vom Mesothorakalganglion, Aufbereitung und Analyse unter dem Transmissionselektronenmikroskop

Zunächst wurden die drei Thorakalganglien einer Heuschrecke zusammenhängend herauspräpariert (siehe Abschnitt 2.5.2) und in eine mit Ringer-Lösung gefüllte Glasschale überführt. Dort wurden alle Konnektive knapp ober- und unterhalb des Mesothorakalganglions abgetrennt und letzteres durch Entfernung von noch anhaftendem Fett- und Tracheengewebe sauber heraus präpariert. Die Aufbereitung und Einbettung der Präparate erfolgte nach der unter Abschnitt 2.8.4 beschriebenen Methode. Dabei wurde eine Primärfixierung in Glutaraldehydlösung und eine Nachfixierung in Osmiumtetroxid- und Natriumcacodylatlösung durchgeführt. Nachdem die Einbettung in Araldit erfolgt war, wurden zunächst Semi-Dünnschnitte des interessierenden Bereiches vom Mesothorakalganglion dicht oberhalb der Mittellinie entnommen und unter dem Lichtmikroskop kontrolliert. Sobald die dorsalen Kommissuren deutlich erkennbar waren, wurden Ultra-Dünnschnitte vom verbleibenden Präparat in dieser Region entnommen und für die TransmissionsElektronenmikroskopie weiter vorbereitet und auf spezielle Objektträger („Grids“) überführt. Mit dem Transmissions-Elektronenmikroskop EM900 (Firma Zeiss) wurden die Schnittserien analysiert und von geeigneten Regionen, in welchen die dorsale Kommissur $\mathrm{DCl}$ gut zu erkennen war, wurden Fotografien bei verschiedenen Vergrößerungen angefertigt. Die entwickelten Negative konnten dann mit einem Scanner (Epson Perfection 1640SU, mit Aufsatz für Negative) unter Benutzung der Scansoftware Epson Twain 5 eingescannt und mit der Bildbearbeitungssoftware Adobe Photoshop CS (Version 8.0.1) für die Auswertung optimiert werden. 


\subsection{Verwendete Lösungen}

\subsubsection{Ringer-Lösung nach CLEMENTS und MAY (1974)}

Die Agenzien werden in destilliertem Wasser gelöst. Die Saccharose wird aber erst kurz vor Gebrauch der Lösung zugesetzt. Das $\mathrm{CaCl} 2$ wird getrennt von den anderen Agenzien in einer Hälfte Aqua dest. gelöst, die anderen Substanzen in die andere Hälfte gegeben. Erst nachdem alle Chemikalien in Lösung übergegangen sind, werden beide Hälften vermischt. Die Lösung hat einen $\mathrm{pH}-$ Wert von $\mathrm{pH}=6,8$.

\begin{tabular}{|l|l|r|r|}
\hline Agens & $\begin{array}{l}\text { Konzentration } \\
\text { [mmol] }\end{array}$ & G/l & g/l (hydr.) \\
\hline $\mathrm{NaCl}$ & 140 & 8,20 & 8,20 \\
\hline $\mathrm{KCl}$ & 10 & 0,75 & 0,75 \\
\hline $\mathrm{CaCl}$ & 2 & 0,22 & 0,29 \\
\hline $\mathrm{NaH}_{2} \mathrm{PO}_{4}$ & 4 & 0,48 & 0,51 \\
\hline $\mathrm{Na}_{2} \mathrm{HPO}_{4}$ & 6 & 0,85 & 1,06 \\
\hline Saccharose & 90 & 30,82 & 30,82 \\
\hline
\end{tabular}

\subsubsection{Paraformaldehyd, 4\%ige Lösung}

4 Gramm reines Paraformaldehyd (Aldrich 15,812-7) werden in einen 100mlSchüttelkolben gegeben und in $20 \mathrm{ml}$ destilliertem Wasser gelöst. Die Lösung wird auf einem Magnetrührer bis auf $60^{\circ}$ erhitzt. Anschließend wird tropfenweise $2 \mathrm{~N}$ $\mathrm{NaOH}$ zugesetzt, bis die Lösung fast klar wird. Nach Abkühlung wird die Lösung mit PBS auf $100 \mathrm{ml}$ aufgefüllt, filtriert und bis zum Gebrauch im Kühlschrank gelagert.

\subsubsection{Phosphate buffered saline (PBS Puffer; $\mathrm{pH}=7,4$ )}

Um einen 0,1M PBS Puffer herzustellen werden 14,19g Na $2 \mathrm{HPO}_{4}, 15,6 \mathrm{~g} \mathrm{NaH} \mathrm{PO}_{4}$ und $4,5 \mathrm{~g} \mathrm{NaCl}$ in $1 \mathrm{~L}$ destilliertem Wasser gelöst. 
2.8.4 Fixierung und Einbettung von medianen Sagittalschnitten (Mesothorakalganglion) für die Transmissionselektronenmikroskopie

\section{Primärfixierung:}

Glutaraldehyd (25\%ig) wird im Verhältnis 1:7 mit 0,08M Cacodylatpufferlösung versetzt. Die Cacodylatpufferlösung enthält 3,4\% Saccharose $(1,712 \mathrm{~g}$ Cacodylat und $3,4 \mathrm{~g}$ Saccharose werden in $100 \mathrm{ml}$ destilliertem Wasser gelöst) und hat einen $\mathrm{pH}$ Wert von $\mathrm{pH}=7,2$.

Die freipräparierten Mesothorakalganglien werden in dieser Lösung über Nacht im Kühlschrank vorfixiert.

\section{Nachfixierung:}

4\%ige Osmiumsäurelösung werden im Verhältnis 1:1 mit 0,08M Cacodylatpufferlösung $(3,4 \%$ Saccharose, $\mathrm{pH}=7,2)$ versetzt. Die Nachfixierung der Präparate in dieser Lösung erfolgt für eine Dauer von 2 Stunden bei Raumtemperatur.

Nach der Entwässerung in einer aufsteigenden Acetonreihe erfolgt die Einbettung in Araldit. 


\subsection{Liste der verwendeten Abkürzungen}

\begin{tabular}{|c|c|c|}
\hline & aLAC & anterior lateral association centre; anterior-laterales Neuropil \\
\hline & aVAC & anterior ventral association centre; anterior-ventrales Neuropil \\
\hline & $\mathrm{DC}$ & dorsal commissure; dorsale Kommissur \\
\hline & DCl-DCVI & dorsale Kommissuren I-VI \\
\hline & $\mathrm{dDCl}$ & $\begin{array}{l}\text { dorsal commissure I, dorsal part } \\
\text { dorsal gelegener Teil der dorsalen Kommissur I }\end{array}$ \\
\hline & $\mathrm{vDCl}$ & $\begin{array}{l}\text { dorsal commissure I, ventral part } \\
\text { ventral gelegener Teil der dorsalen Kommissur I }\end{array}$ \\
\hline & DIT & $\begin{array}{l}\text { dorsal intermediate tract; dorsal-intermediärer Trakt } \\
\text { (longitudinal) }\end{array}$ \\
\hline & DMT & dorsal median tract; dorsal-medianer Trakt (longitudinal) \\
\hline & LDT & lateral dorsal tract; lateral-dorsaler Trakt (longitudinal) \\
\hline & IVAC & lateral ventral association centre; lateral-ventrales Neuropil \\
\hline & MDT & median dorsal tract; median-dorsaler Trakt (longitudinal) \\
\hline & mVAC & median ventral association centre; median-ventrales Neuropil \\
\hline & MVT & median ventral tract; median-ventraler Trakt (longitudinal) \\
\hline & pLAC & $\begin{array}{l}\text { posterior lateral association centre; posterior-laterales } \\
\text { Neuropil }\end{array}$ \\
\hline & SMC & supramedian commissure; supramediane Kommissur \\
\hline & TT & T-tract; T-Trakt (vertikal) \\
\hline & VIT & $\begin{array}{l}\text { ventral intermediate tract; ventral-intermediärer Trakt } \\
\text { (longitudinal) }\end{array}$ \\
\hline & VLT & ventral lateral tract; ventral-lateraler Trakt (Iongitudinal) \\
\hline & VMT & ventral median tract; ventral-medianer Trakt (longitudinal) \\
\hline & VVAC & $\begin{array}{l}\text { ventralmost ventral association centre; äußerst ventral } \\
\text { gelegenes ventrales Neuropil }\end{array}$ \\
\hline
\end{tabular}




\begin{tabular}{|c|c|c|}
\hline \multirow{10}{*}{ 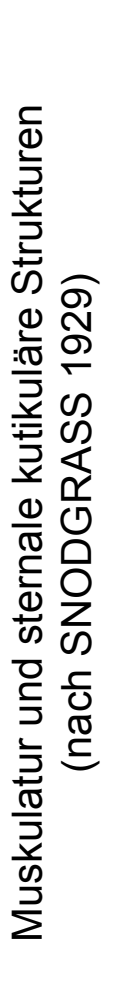 } & M60 & zweiter ventraler Längsmuskel \\
\hline & M67 & zweiter posteriorer Rotator der Coxa (Vorderbein) \\
\hline & M87 & dritter ventraler Längsmuskel \\
\hline & M88 & vierter ventraler Längsmuskel \\
\hline & M93 & posteriorer Rotator der Coxa (Mittelbein) \\
\hline & M96 & dritter Abduktor der Coxa (Mittelbein) \\
\hline & M103a & $\begin{array}{l}\text { Depressor trochanteris; coxaler Teil des Depressors des } \\
\text { Trochanters }\end{array}$ \\
\hline & M117 & Fünfter ventraler Längsmuskel \\
\hline & 1Spn & prothorakale sternale Spina \\
\hline & 2Spn & mesothorakale sternale Spina (Apophyse) \\
\hline
\end{tabular}

\begin{tabular}{|c|c|c|}
\hline \multirow{5}{*}{ 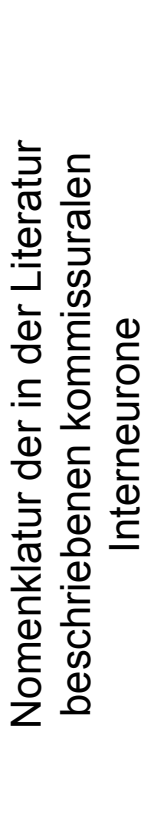 } & $\mathrm{aCIN}$ & $\begin{array}{l}\text { ascending commissural interneuron; Kommissurales } \\
\text { Interneuron mit einem im Konnektiv aufsteigenden Axon }\end{array}$ \\
\hline & $\mathrm{dCIN}$ & $\begin{array}{l}\text { descending commissural interneuron; Kommissurales } \\
\text { Interneuron mit einem im Konnektiv absteigenden Axon }\end{array}$ \\
\hline & $\operatorname{adCIN}$ & $\begin{array}{l}\text { ascending-descending commissural interneuron; } \\
\text { Kommissurales Interneuron mit Bifurkation (Axon sowohl auf- } \\
\text { als auch absteigend }\end{array}$ \\
\hline & ICIN & $\begin{array}{l}\text { longe range commissural interneuron; Intersegmentales } \\
\text { kommissurales Interneuron }\end{array}$ \\
\hline & sCIN & $\begin{array}{l}\text { short range commissural interneuron; Lokales kommissurales } \\
\text { Interneuron (Es gibt auch intersegmentale Neurone in dieser } \\
\text { Klasse, die sich aber höchstens bis über 1,5 benachbarte } \\
\text { Segmente erstrecken) }\end{array}$ \\
\hline
\end{tabular}




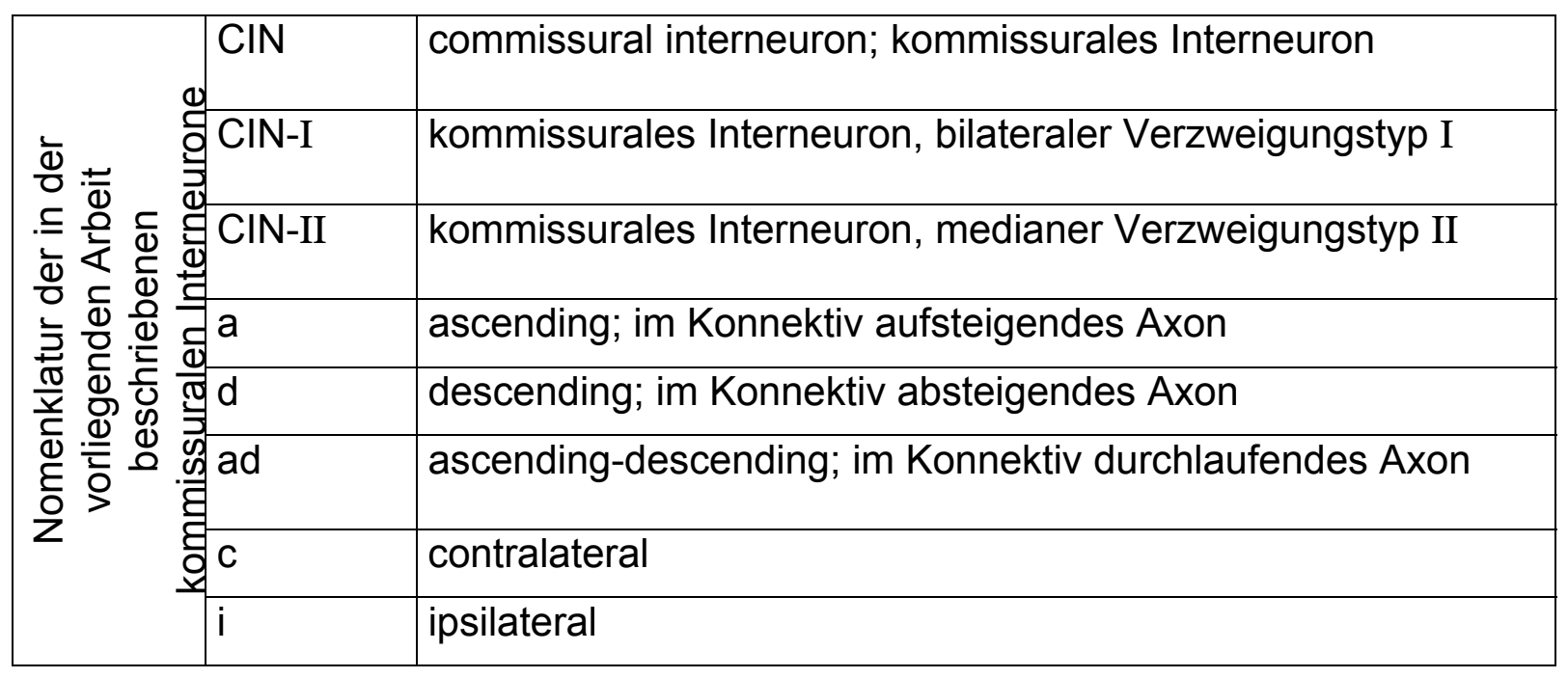

\begin{tabular}{|c|c|c|}
\hline \multirow{16}{*}{ 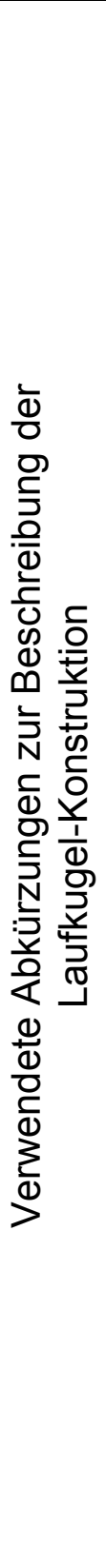 } & BL & $\begin{array}{l}\text { Holzblöckchen mit integrierter Glashülse als Lagerung für die } \\
\text { Achsen-Nadeln (Drehachse der Laufkugel) }\end{array}$ \\
\hline & $\mathrm{F}$ & Fett- und Nährgewebe und Tracheen \\
\hline & G & Gummifaden \\
\hline & $\mathrm{H}$ & Halterung für Gummifaden (Ösenschraube) \\
\hline & $\mathrm{HZ}$ & Holzblock (Sockel der Laufkugel-Konstruktion) \\
\hline & I & intrazelluläre Ableitung (Siehe auch IN) \\
\hline & L & Laufkugel aus Styropor \\
\hline & $\mathrm{M}$ & Muskelgewebe im Präparat (soweit nicht weiter differenziert) \\
\hline & $\mathrm{N}$ & Achsen-Nadeln (Drehachse der Laufkugel) \\
\hline & R & Gelenkrolle (Glashülse) \\
\hline & S & Stützdraht (Aufhängung der Laufkugel-Konstruktion) \\
\hline & TA & Tracheen, Tracheenstamm \\
\hline & T2 & Mesothorakalganglion (Siehe auch MsG) \\
\hline & T3 & Metathorakalganglion (Siehe auch MtG) \\
\hline & VN & $\begin{array}{l}\text { Drehbarer Untersatz für die Laufkugel-Konstruktion (Ventilator, } \\
\text { PC-Gehäuse-Lüfter) }\end{array}$ \\
\hline & W & Wachsabdeckung (Bienenwachs-Kolophonium Gemisch) \\
\hline
\end{tabular}




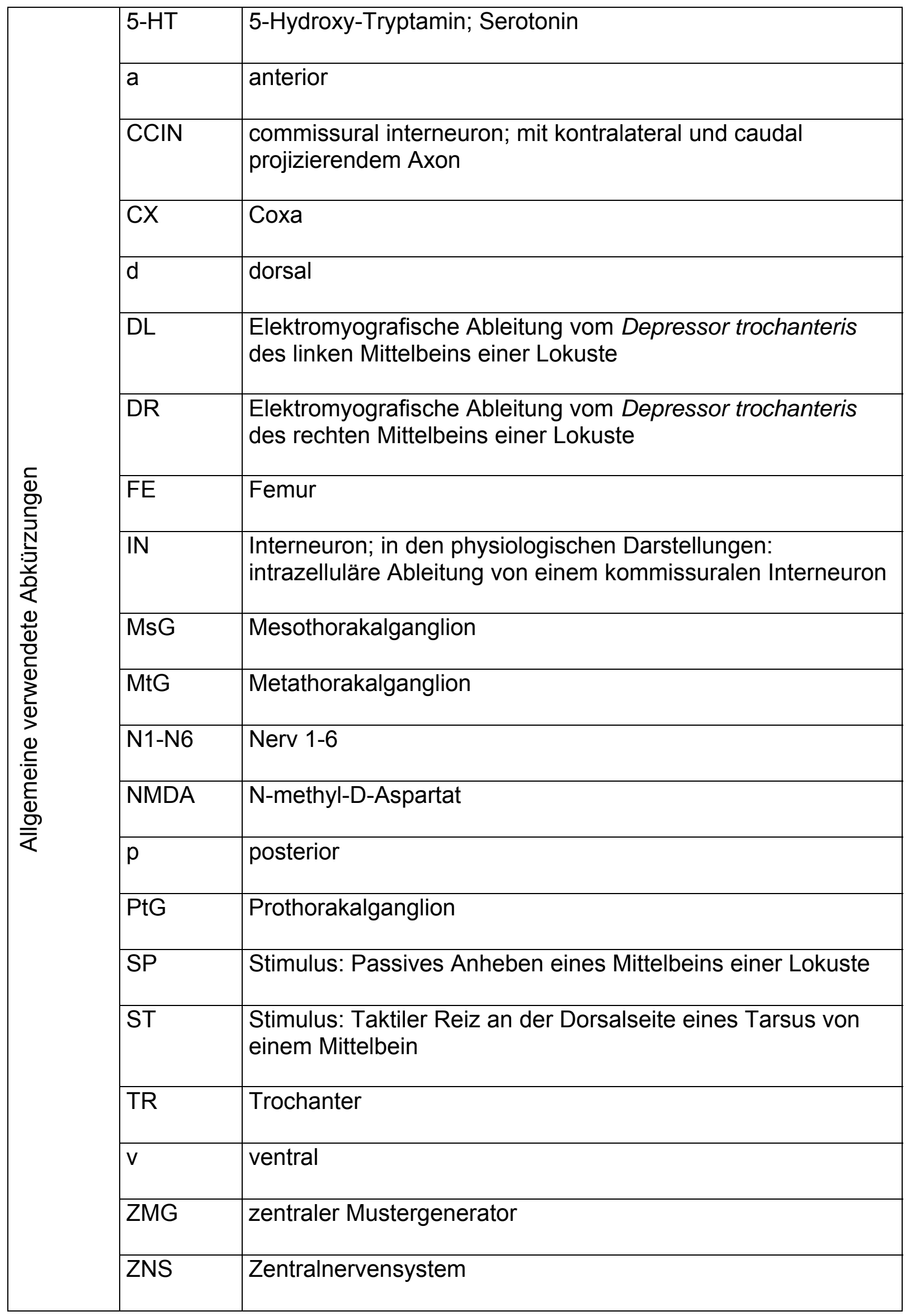


Vorbemerkungen:

Das Ziel der vorliegenden Arbeit ist es, kommissurale Interneurone (CIN) des Mesothorakalganglions zu beschreiben, die zur bilateralen Koordination der beiden Mittelbeine der Wanderheuschrecke Locusta migratoria beitragen. Es werden im Folgenden zwei Gruppen von Interneurontypen morphologisch wie physiologisch beschrieben: Typ CIN-I und Typ CIN-II Neurone, die Eigenschaften einer die Extremitäten koordinierenden Funktion haben und bei intrazellulären Ableitungen in der Region der dorsalen Kommissuren DCI-DCVI häufig vorzufinden sind.

Im Abschnitt 3.1 werden zunächst sieben der insgesamt sechzehn in den Übersichten gezeigten Neuronen (Übersichten 1-4, Abb. 33-36, Abschnitt 3.2) detailliert vorgestellt. Hier erfolgt nach einer Beschreibung ihrer Morphologie und Physiologie eine anschließende Kurzdiskussion separat für jedes einzelne Neuron. Die dort dargestellten Erkenntnisse werden später in der allgemeinen Diskussion wieder aufgegriffen und mit der in diesem Zusammenhang relevanten Literatur diskutiert. Im Abschnitt $\mathbf{3 . 2}$ erfolgt eine Beschreibung von allgemeinen morphologischen wie physiologischen Merkmalen beider Neurontypen, und zwar jeweils im Zusammenhang mit Übersichts-Abbildungen (Abb. 33-36), die eine Auswahl von charakteristischen Interneuronen aus der jeweiligen Gruppe veranschaulichen sollen. Bei allen hier vorgestellten kommissuralen Interneuronen mit Charakteristika einer die Extremitäten koordinierenden Funktion handelt es sich um intersegmentale Interneurone, die jeweils durch eine der dorsal gelegenen Kommissuren DCI-DCVI verlaufen. Gemäß der Vermutung, dass lokale bilateral koordinierende Interneurone durch die dorsale Kommissur DCl verlaufen, wurde versucht Zellen hauptsächlich in dieser Region zu penetrieren. Eine solche über längere Zeit stabile Ableitung gelang jedoch nur in wenigen Fällen. Auch konnten innerhalb dieser Kommissur keine lokalen Interneurone mit Eigenschaften einer bilateral koordinierenden Funktion physiologisch und morphologisch erfolgreich charakterisiert werden. Das wirft die Frage nach der äußeren Begrenzung dieser Kommissur DCl und nach der Anzahl der sie passierenden Zellen auf. In diesem 
Zusammenhang wurden sagittale Schnittserien vom Mesothorakalganglion (Ultradünnschnitte) im kommissuralen Bereich auf Höhe der Mittellinie angefertigt und für die Elektronenmikroskopie aufbereitet. Die Ergebnisse sind im Abschnitt $\mathbf{3 . 3}$ dargestellt.

3.1 Detaillierte Beschreibung von morphologischen und physiologischen Merkmalen von ausgewählten Typ CIN-I- und Typ CIN-II Interneuronen

Im Folgenden werden insgesamt sieben ausgewählte Neurone detailliert vorgestellt, vier vom bilateralen Verzweigungstyp CIN-I und drei vom medianen VerzweigungsTyp CIN-II. Die Beschreibung erfolgt für jedes Neuron separat und gliedert sich in die Abschnitte Morphologie, Physiologie und Kurzdiskussion. Letztere soll aufgrund der morphologischen und physiologischen Eigenschaften des betreffenden Neurons eine Abschätzung darüber geben, ob eine bilateral die Extremitäten koordinierende Funktion in Betracht gezogen werden kann und welche Rolle das kommissurale Interneuron daneben im Kontext der allgemeinen Koordination der Extremitäten (intersegmental und/oder bilateral) spielen könnte.

Die in jedem Morphologieteil verwendeten Abbildungen zeigen zuerst eine gezeichnete, zweidimensionale Rekonstruktion des betreffenden Neurons im Mesothorakalganglion mit jeweils darunter liegender Vergleichsübersicht, in welcher Verzweigungsstrukturen des Neurons zu bekannten morphologischen Strukturen innerhalb dieses Ganglions (relative Lage von dorsalen Kommissuren und mechanosensorischen Neuropilen) zugeordnet werden können (nach PFLÜGER et al. 1988; leicht modifiziert). Die jeweils darauf folgende Abbildung zeigt eine Originalfotografie desselben Neurons im Mesothorakalganglion an einer markanten Stelle seiner Verzweigungsstruktur. Falls in der Färbung eines Neurons auch Teile der metathorakalen oder prothorakalen Verzweigungen zu rekonstruieren sind, so findet sich in selbiger Abbildung neben einer weiteren Originalfotografie der entsprechenden Struktur auch wieder die dazugehörige zweidimensional gezeichnete Rekonstruktion, die dann in die Zuordnungsübersicht mit den bekannten morphologischen Strukturen integriert ist. 
In den Abbildungen des jeweils zum betreffenden Neuron dazugehörigen Physiologieteils werden originale Ausschnitte der aufgezeichneten neuronalen Aktivität (IN) zusammen mit den parallel registrierten Muskelaktivitäten des rechten (DR) und linken (DL) Depressor trochanteris (M103a, nach SNODGRASS, 1929) im Kontext von durch Reizung ausgelösten Reflexen oder während aktiver Bewegung der Mittelbeine (ohne Reizung) dargestellt. Der Zeitpunkt der Reizungen ist in einer separaten Spur, der Reizindikation (ST, SP) angegeben. In diesem Zusammenhang ist anzumerken, dass sich bei manchen Präparaten Reflexe (bilaterale und/oder intersegmentale) meist erst nach wiederholter Reizung sichtbar auslösen ließen (sichtbare Bewegung der Extremitäten). Die Reizindikation (ST) ist in diesem Fall dann als Beginn einer kontinuierlichen Reizung bis zum Reizerfolg (Bewegung) zu verstehen und nicht als zeitlich genau zugeordnete Einzelreizung. In der betreffenden Spur der Reizindikation ist der Zeitraum der Reizung dann durch eine gestrichelte Linie angegeben. In solchen Fällen, in welchen die durch Reizung ausgelösten Extremitätenbewegungen und gemessenen Muskelaktivitäten sehr schwach waren, wurden auch mechanische Reize durch passives Anheben des betreffenden Mittelbeins von der Ventralseite der Tibia her gegeben (SP). Die Möglichkeit, dass die durch eine solche Auslenkbewegung entstehenden Kräfte auch mechanisch Veränderungen der intrazellulären Ableitbedingungen bewirken können, wird an den entsprechenden Stellen berücksichtigt. Zur Verdeutlichung der Veränderungen der neuronalen Aktivität in Korrelation zu den gemessenen Muskelaktivitäten werden die Instantan- und die Spontanfrequenz des Neurons in einem entsprechenden Histogramm in zeitlicher Synchronisation mit den Originalspuren gezeigt. Dabei sind immer die Werte der Minimal- und Maximal-Frequenz innerhalb des Ausschnittes angegeben. Die Spontanfrequenz des Neurons zum Zeitpunkt kurz vor der Reizung ist durch eine horizontale Linie auf dem entsprechenden FrequenzNiveau angegeben. 


\subsubsection{Bilateral verzweigtes kommissurales Typ CIN-I Interneuron CIN-I(ad)c1}

Morphologie:

\section{Mesothorakalganglion:}

Die Abbildungen 6-7 zeigen das Interneuron $\mathrm{CIN}-\mathrm{I}(\mathrm{ad}) \mathrm{c} 1$, ein im linken Konnektiv des Mesothorakalganglions intermediär durchlaufendes, intersegmentales Interneuron mit bilateralem Verzweigungsmuster. Das Soma liegt ventral und anterior-lateral im

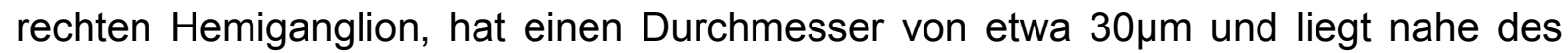
rechten aufsteigenden Konnektivs (gedachte Verbindungslinie entlang der lateralen Randzone der beiden sich longitudinal gegenüberliegenden Konnektive). Der primäre Neurit verläuft nach posterior und steigt bis auf medianes Niveau an. Dabei nähert er sich zunächst der Mittellinie, verläuft dann aber auf Höhe der dorsalen Kommissur $\mathrm{DCl}$ bogenförmig nach anterior in Richtung derselbigen.

Unmittelbar vor Passieren der Kommissur entspringt die erste Hauptverzweigung nach ipsilateral und verläuft nach anterior-lateral, wo sie sich zunächst auf medianem Niveau weiter aufteilt. Während der erste Abzweig nach posterior in ventrale Richtung zieht, setzen sich alle folgenden weiter nach anterior fort und steigen bis nach dorsal auf. Der primäre Neurit passiert dann im folgenden Abschnitt zum ersten Mal die dorsale Kommissur DCl. Nahe der Mittellinie entspringt ein kleinerer Abzweig, der sich nach kontralateral fortsetzt, zunächst dorsal etwas ansteigend, sich dann aber bogenförmig zunehmend nach ventral verlagernd und schließlich auf der Höhe relativ zur inneren Randzone der kontralateralen (linken) Konnektive (gedachte longitudinale Verbindungslinie) ein kurzes Stück nach posterior ziehend.

Die nächsten beiden sich gegenüber liegenden Hauptverzweigungen (2 und 3 ) entspringen dem primären Neuriten auf kontralateraler Seite. Eine verläuft ebenfalls nach posterior auf medianem Niveau und in der Nähe der Mittellinie. Die andere zieht nach anterior-lateral und teilt sich auf medianer Höhe in mehrere Fortsätze, die sich alle zunehmend nach lateral verlagern. Alle verzweigen bis weit nach dorsal, so dass ein weiter, dorsal-lateraler Bereich des linken Hemiganglions abgedeckt wird.

Der primäre Neurit verläuft auf kontralateraler Seite bogenförmig nach dorsal. Vom Scheitelpunkt dieses Bogens entspringt das Axon, das zunächst auf medianer Höhe ein Stück weit nach lateral zieht und sich dann in zwei Hauptkollaterale des Axons aufteilt. Eines steigt im linken Konnektiv intermediär auf und besitzt eine kleinere 
mediane Kollaterale. Das andere steigt im linken Konnektiv intermediär ab und trägt mehrere kleine, aber auch zwei größere Kollaterale. Während die kleineren alle median verzweigen, setzen sich die beiden größeren auch bis weit nach dorsal fort. Die größte Kollaterale verläuft in mediane Richtung mit einem kontralateralen Abzweig im linken Hemiganglion (medianes Niveau). Der andere Teil nähert sich median der Mittellinie, durchquert schließlich eine der hinteren dorsalen Kommissuren (DCIV-DCV) und verzweigt sich im rechten Hemiganglion bis weit nach lateral und dorsal (etwa bis auf Höhe der gedachten Verbindungslinie zwischen Nerv 1 und Nerv 6). Die zweitgrößte Kollaterale zieht nach lateral (kontralateral, linkes Hemiganglion) und verzweigt ebenfalls in dorsaler Richtung.

Nach dem Abzweig des Axons vom primären Neuriten verläuft dieser weiterhin bogenförmig und zieht schließlich zurück in Richtung der Mittellinie, wo er die vorderste dorsale Kommissur DCl ein weiteres Mal (etwas weiter dorsal) passiert. Dieser kommissurale Fortsatz teilt sich dann auf ipsilateraler Seite in die Hauptverzweigung 4, eine mittlere und eine kleine Nebenverzweigung. Die mittlere Seitenverzweigung zieht nach anterior und teilt sich in einen ipsilateralen Teil, der weiter nach anterior-lateral zieht und dorsal verzweigt, sowie in einen kontralateralen Teil, der die vorderste Kommissur durchquert und dann im mittleren Feld ebenfalls dorsal verzweigt. Die Hauptverzweigung 4 zieht nach posterior und teilt sich dann in einen Teil, der ebenfalls anterior und dorsal-lateral verzweigt, sowie einen zweiten, der zunächst weiter nach posterior zieht und dann dorsal-lateral über einen weiten Bereich des rechten Hemiganglions verzweigt.

Die beiden bilateralen Verzweigungsfelder sind zwar nicht exakt symmetrisch organisiert, sie ähneln sich aber in gewissem Maße. Der Großteil ihrer Verzweigungen ist sehr weit dorsal gelegen, keines der bilateralen Felder reicht extrem weit nach lateral, so dass sie nur knapp an die dorsalen Neuropile (pLAC, aLAC) heranreichen. An vielen, aber nicht an allen der feineren dorsalen Verzweigungen lassen sich über den gesamten Bereich auch zahlreiche kleine Varikositäten finden. Möglicherweise liegen hier Input- und Outputregionen dicht beieinander.

\section{Pro- und Metathorakalganglion:}

Die Situation im Pro- und Metathorakalganglion ist aufgrund hier unvollständiger Färbung nicht bekannt. 


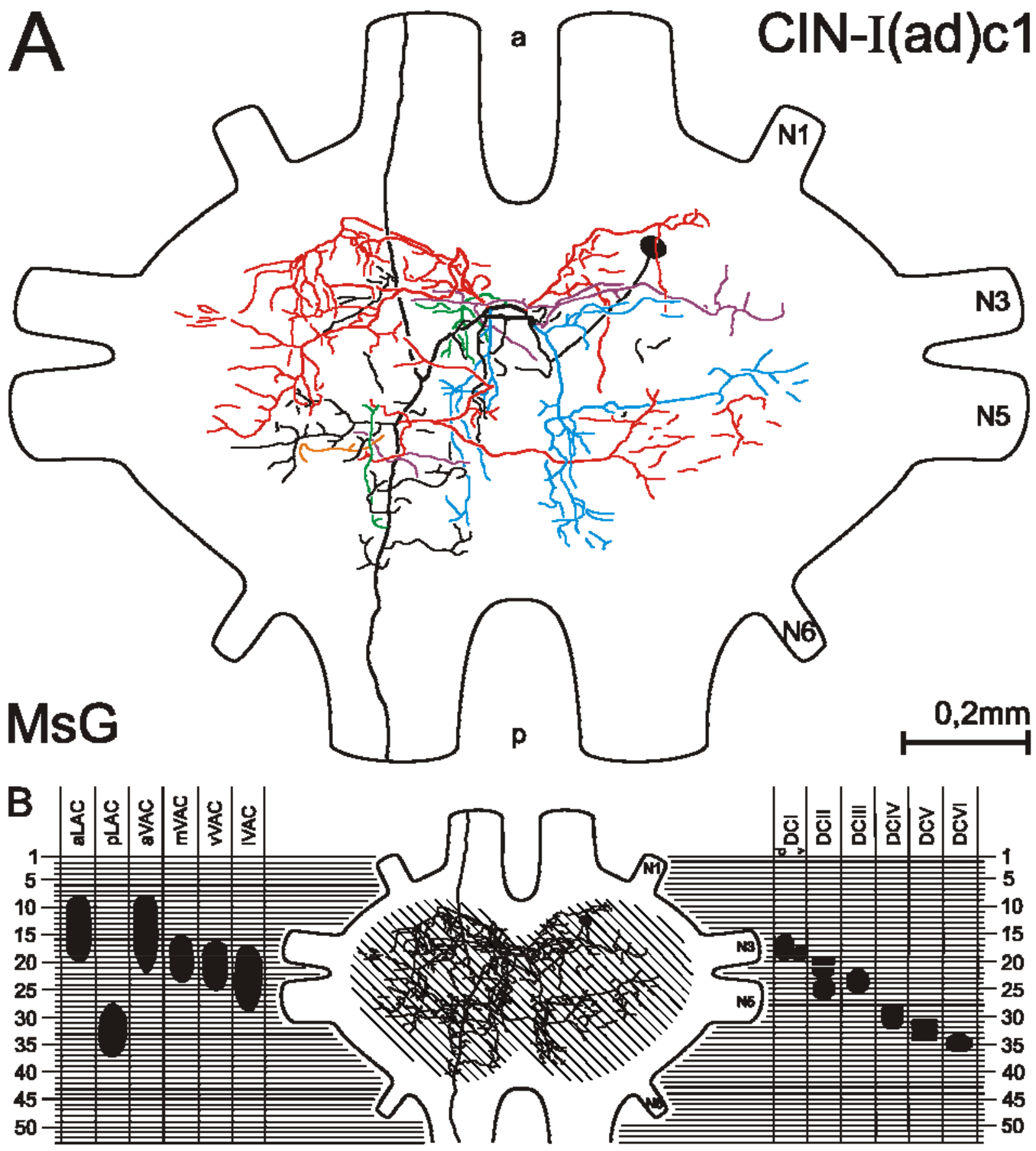

Abb. 6: Intersegmentales Interneuron CIN-I(ad)c1 mit bilateralem Verzweigungsmuster (Typ I). A: Gezeichnete 2D-Projektion, Mesothorakalganglion. Intermediär im kontralateralen (linken) Konnektiv durchlaufendes Axon. Die meisten Verzweigungen erstrecken sich dorsal-median bis dorsal-lateral über einen weiten Bereich in beiden Hemiganglien und reichen knapp an die dorsalen Neuropile ( $\mathrm{DLAC}, \mathrm{aLAC}$ ) heran. Einige Ausläufer ziehen auch nach ventral oder in den intermediären Bereich. Der absteigende Teil des Axons trägt mehrere zum Teil sehr weitreichende Kollaterale, deren größte dorsal über eine der dorsalen Kommissuren DCIV-DCV bis in das gegenüber liegende Hemiganglion zieht, wo sie mit lokalen Verzweigungen überlappt. Der primäre Neurit passiert die dorsale Kommissur $\mathrm{DCl}$ zweimal und hat kontralateral einen bogenförmigen Verlauf, von dessen Scheitelpunkt das Axon entspringt. Das Soma $(\varnothing=30 \mu \mathrm{m})$ liegt lateral und anterior-ventral. B: Übersicht desselben Neurons und Vergleich mit der relativen Lage wichtiger morphologischer Strukturen (aus: PFLÜGER et al. 1988; leicht modifiziert).

Abkürzungen: MsG: Mesothorakalganglion; a: anterior; p: posterior; N1-N6: Nerv1-6; Schraffur in B: Kennzeichnung für vorhandene varikose Fasern (potentiell lokale Projektionsbereiche), die nebeneinander mit dendritischen Fasern vorkommen; Abkürzungen in B Siehe Tabelle der verwendeten Abkürzungen. 


\section{CIN-I(ad)c1}

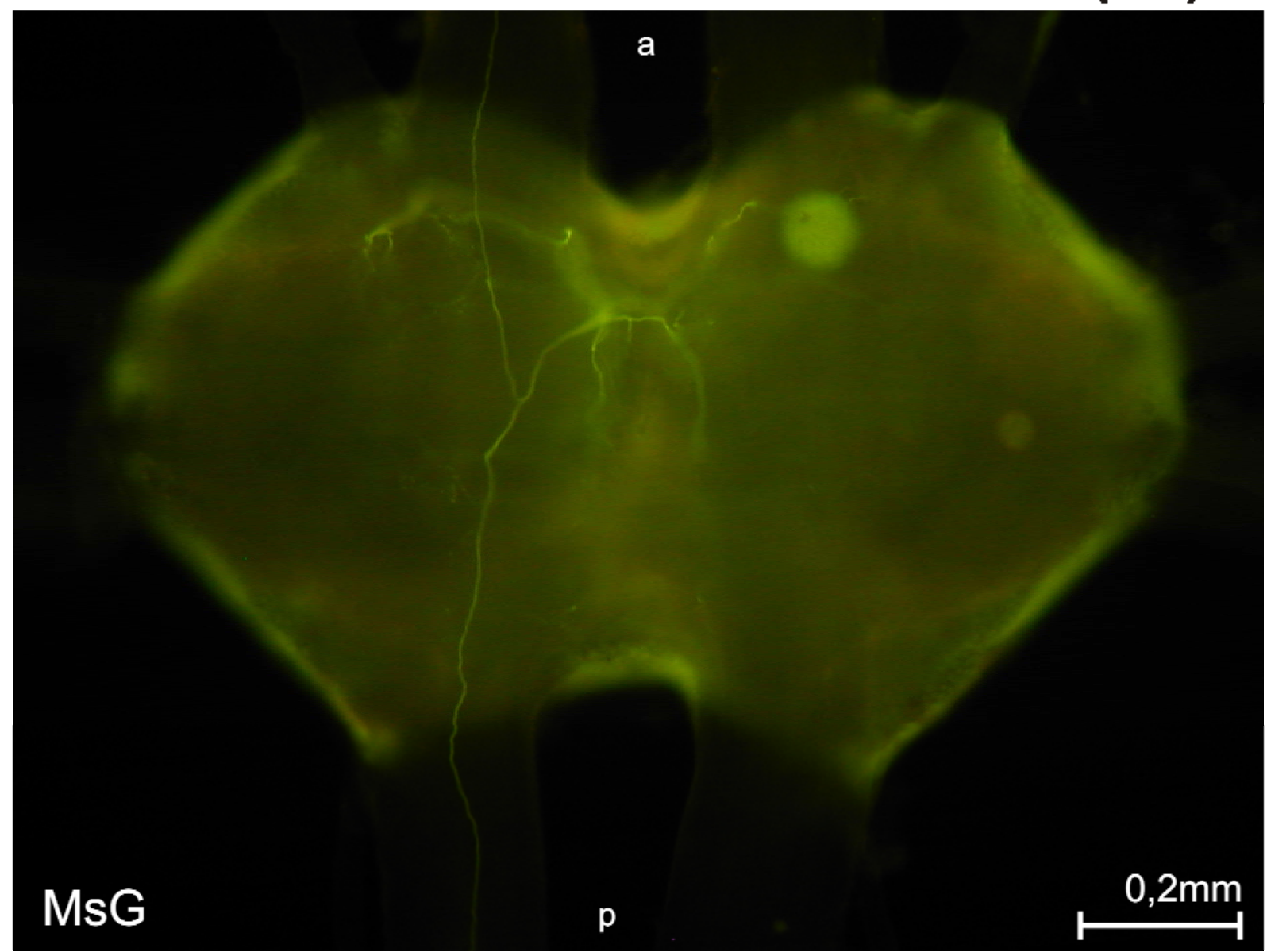

Abb. 7: Originalfotografie des Neurons CIN-I(ad)c1, Mesothorakalganglion. Die Fokusebene befindet sich dorsal, auf der Höhe auf welcher der primäre Neurit die dorsale Kommissur DCI durchtritt. Sowohl der auf- als auch der absteigende Teil des im kontralateralen (linken) Konnektiv durchlaufenden Axons zieht relativ weit dorsal durch das Konnektiv. Median sowie im kontralateralen Hemiganglion sind zum Teil die Strukturen der Hauptverzweigungen und auch der Kollaterale zu erkennen, der Großteil ihrer Verzweigungsbereiche liegt aber noch weiter dorsal und ist hier nicht zu erkennen. Das ventral gelegene Soma fluoresziert diffus in der Tiefe des ipsilateralen (rechten) Hemiganglions.

Abkürzungen: MsG: Mesothorakalganglion; a: anterior; p: posterior.

Physiologie:

Das phasisch-tonische Neuron CIN-I(ad)c1 hat eine Spontanfrequenz zwischen 30$40 \mathrm{~Hz}$ und reagiert mit phasischer Erregung auf dorsal-taktile Reizung des Tarsus des kontralateralen Mittelbeins (relativ zum Soma). Die Reaktion ist nicht sehr stark, dennoch steigt die Instantanfrequenz bei jeder der vier Reizungen einer Sequenz (Abb. 8, A) nach der ersten Reizung kurzfristig über $70 \mathrm{~Hz}$, bei den folgenden jeweils über $50 \mathrm{~Hz}$. Jede dieser kurzen Erregungsphasen erscheint zeitlich unmittelbar 


\section{$\mathrm{CIN}-\mathrm{I}(\mathrm{ad}) \mathrm{c} 1$}

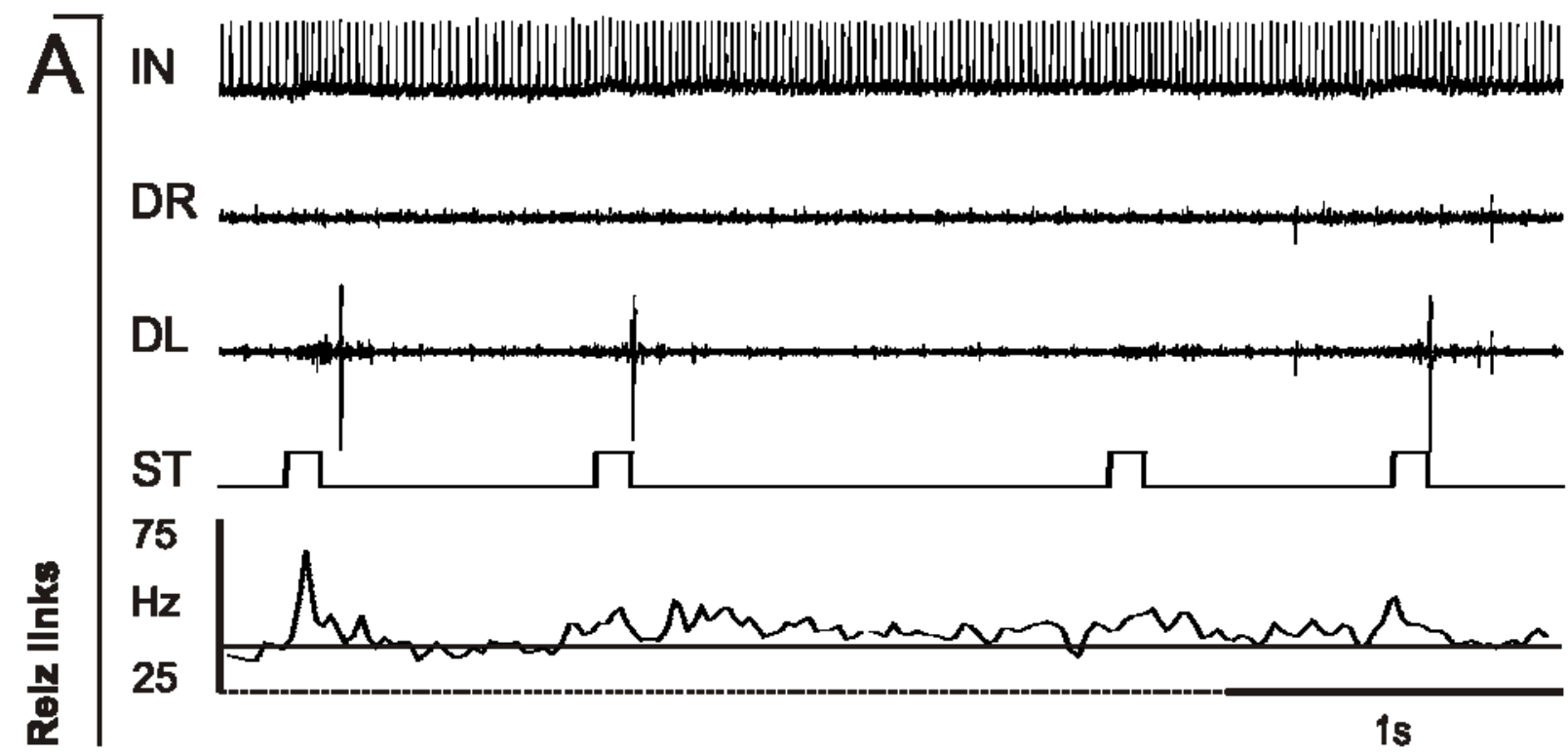

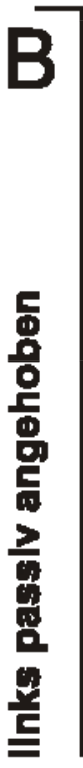

DR

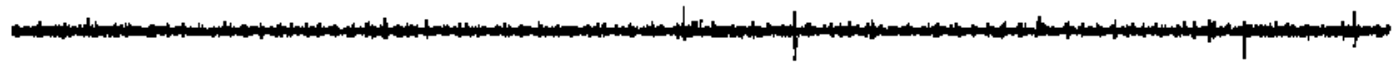

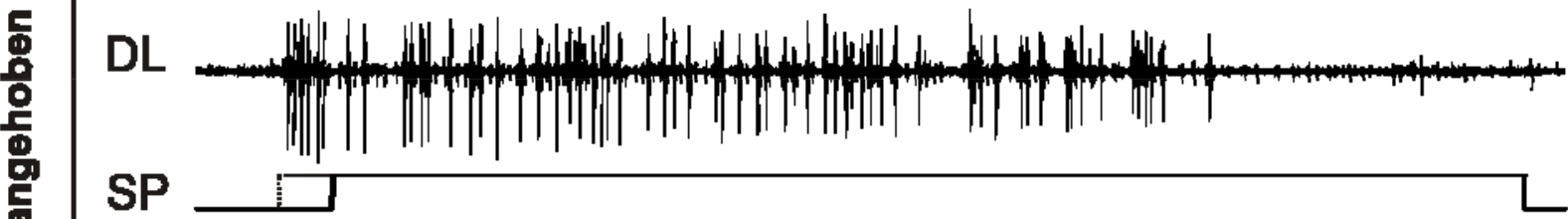

$\mathrm{SP}$

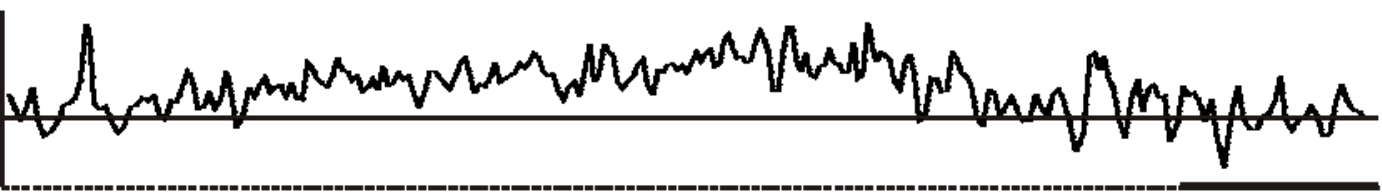

20

1s

Abb. 8: Aktivität, Instantan- und Spontanfrequenzen des Neurons CIN-I(ad)c1 und Muskelaktivitäten bei kontralateraler Reizung (linkes Mittelbein). A: Vierfacher, dorsal-taktiler Tarsusreiz. Bei drei Reizungen ist jeweils eine kurze schwache, phasische Erregung des sonst tonisch (SpontanFrequenz bei knapp $40 \mathrm{~Hz}$ ) aktiven Neurons (IN) erkennbar. Nur in diesen drei Fällen erscheint Muskelaktivität des Depressor trochanteris (linkes Mittelbein, DL). Neuronaktivität erscheint zeitlich jeweils kurz vorher. Nahezu für die gesamte Dauer zwischen den Reizungen erscheint ein andauernder, schwacher Anstieg der Neuronaktivität (liegt dauerhaft oberhalb der Spontanfrequenz). B: Passives Anheben des linken Mittelbeins. Synchron erhöhte Neuronaktivität, abhängig von Dauer und Amplitude der Anhebbewegung. Die Reizindikation erscheint in der Aufzeichnung verzögert $(<0,5 \mathrm{~s}$, Verzögerung durch gestrichelte Linie gekennzeichnet), dadurch erklärt sich die nur scheinbar vor der Reizung auftretende Neuron- und Muskelaktivität. Der Effekt (Erregung des Neurons synchron zur Depressoraktivität DL) ist aber dennoch zu erkennen.

Abkürzungen: IN: Aktivität des Neurons; DR: Depressor trochanteris, rechtes Mittelbein; DL: Depressor tr., linkes Mittelbein; ST: dorsal-taktiler Tarsusreiz; SP: passives mechanisches Anheben. 


\section{CIN-I(ad)c1}
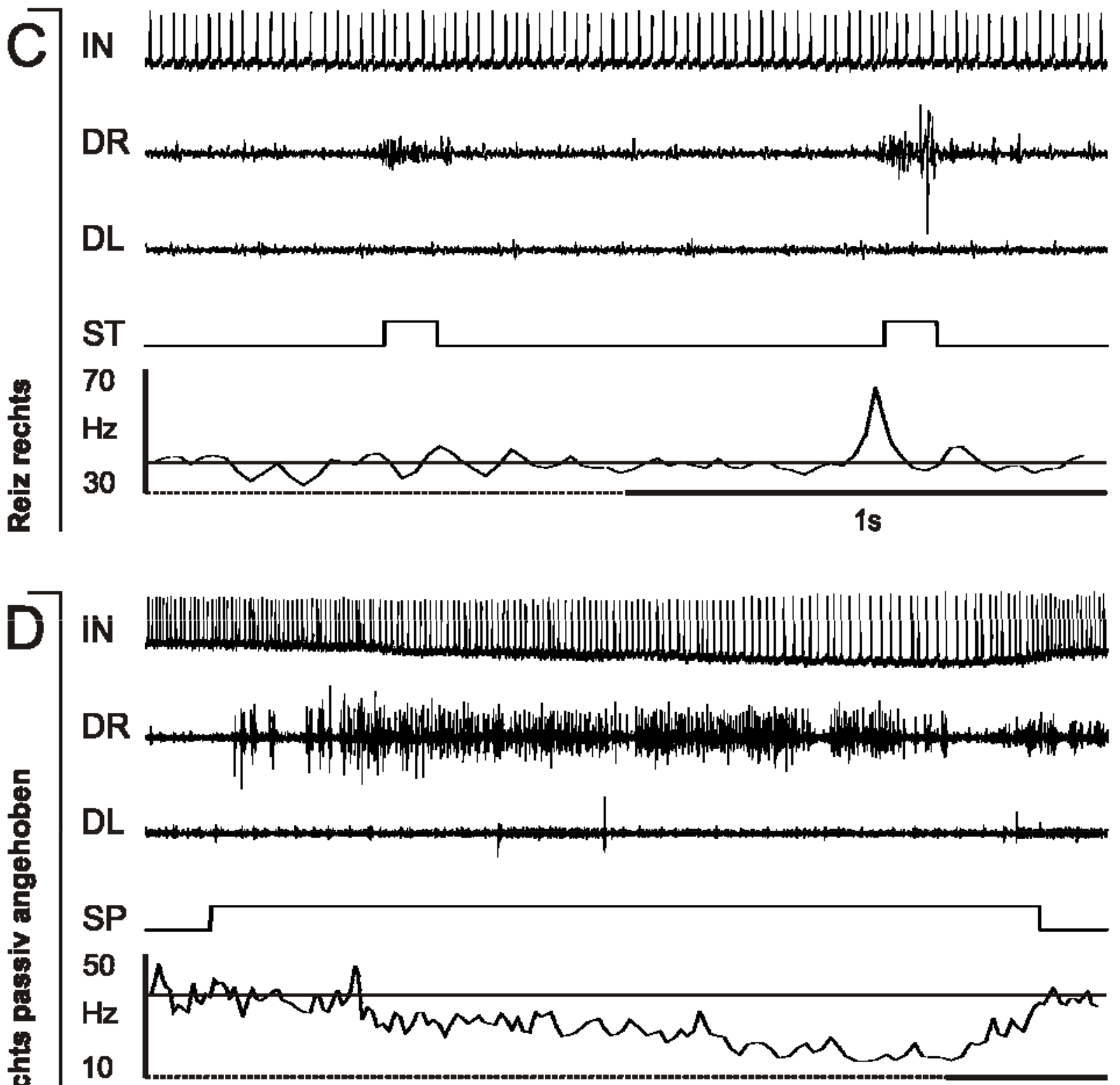

1s

Abb. 9: Aktivität, Instantan- und Spontanfrequenzen des Neurons CIN-I(ad)c1 und Muskelaktivitäten bei ipsilateraler Reizung (rechtes Mittelbein). C: Zweimaliger dorsal-taktiler Tarsusreiz. Die Reizindikation (ST) erfolgt hier geringfügig verzögert (Verzögerung durch gestrichelte Linie gekennzeichnet), dadurch erklärt sich der Eindruck, dass das Neuron (IN) scheinbar bereits vor der Reizung erhöhte Aktivität zeigt. Ein dauerhafter Anstieg der Spikefrequenz zwischen den Reizungen ist nicht erkennbar. Bei der zweiten Reizung erscheint eine kurze, phasisch erhöhte Neuronaktivität (bis knapp $70 \mathrm{~Hz}$ ), die kurz vor der Aktivität des Depressor trochanteris (DR) beginnt und kurz nach Einsetzen der Muskelaktivität beendet ist. D: Passives Anheben vom rechten Mittelbein und Widerstandsreflex. Für die Dauer des Anhebens erfolgt eine zunehmende Hemmung des Neurons, abhängig von der Amplitude des Anhebens. Eine vollständige Hemmung wird jedoch nicht erreicht. Der Widerstandsreflex zeigt sich in der dauerhaften Aktivität des Depressors vom ipsilateralen (rechten) Mittelbein (DR).

Abkürzungen: IN: Aktivität des Neurons; DR: Depressor trochanteris, rechtes Mittelbein; DL: Depressor tr., linkes Mittelbein; ST: dorsal-taktiler Tarsusreiz; SP: passives mechanisches Anheben. 


\section{CIN-I(ad)c1}

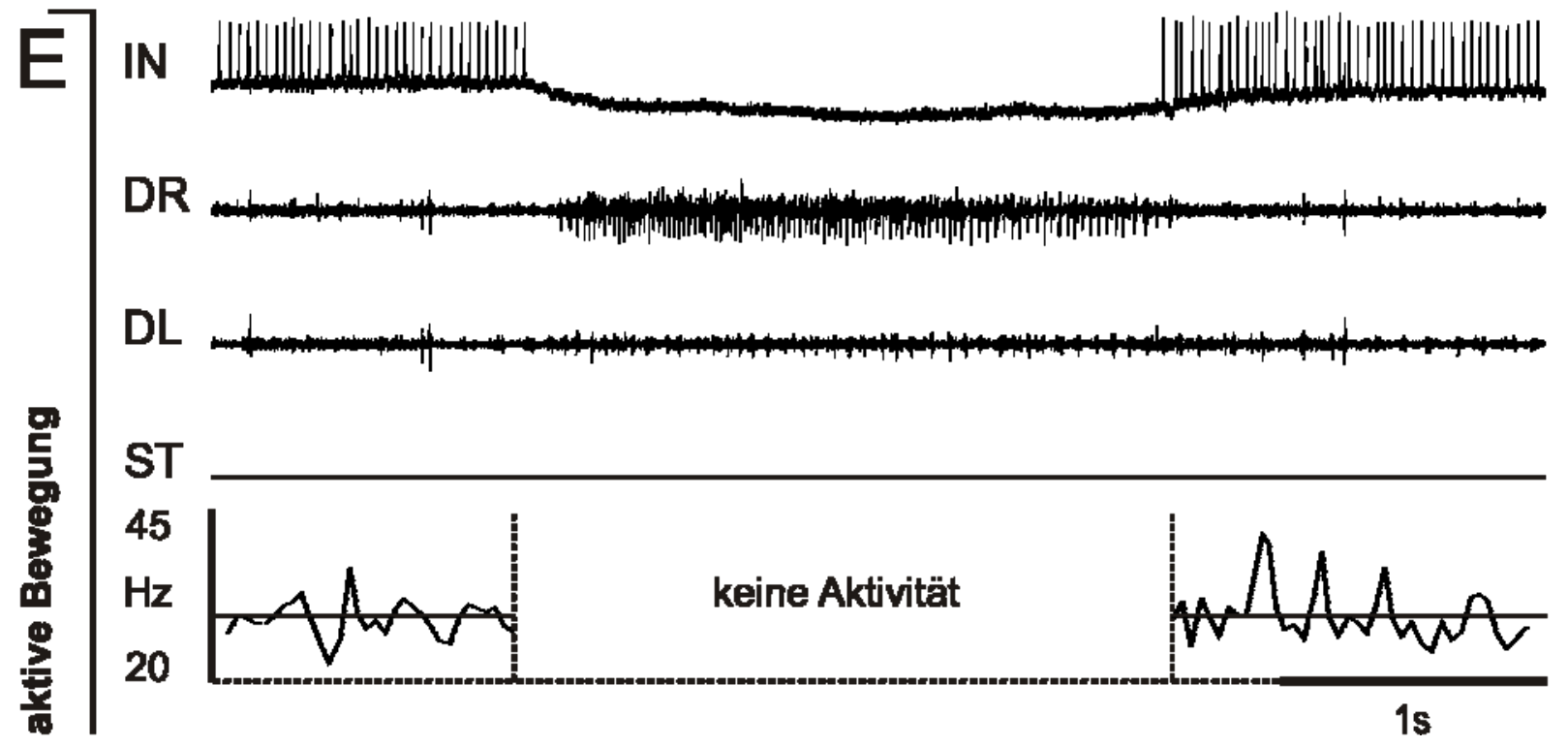

Abb. 10: E: Aktivität, Instantan- und Spontanfrequenzen des Neurons CIN-I(ad)c1 und Muskelaktivitäten bei aktiver Bewegung beider Mittelbeine (nicht gereizt). Beide Depressormuskeln (DR, $\mathrm{DL}$ ) sind hier mehr oder weniger synchron aktiv, daher handelt es sich nicht um eine Laufbewegung. Für die Dauer der Bewegungen erfolgt eine vollständige Hemmung des Neurons (IN). Die Darstellung der Spontan- und Instantanfrequenz ist für diesen Zeitraum unterbrochen, da keine Neuronaktivität erscheint (vertikale gestrichelte Linien).

Abkürzungen: IN: Aktivität des Neurons; DR: Depressor trochanteris, rechtes Mittelbein; DL: Depressor tr., linkes Mittelbein; ST: Reizindikation, keine Reizung.

vor der Muskelaktivität. Daneben erscheint auch ein schwacher, andauernder Anstieg der Spikefrequenz (oberhalb der Spontanfrequenz) nahezu während des gesamten Zeitraums der Folgereizungen. Eine passive, also aufgezwungene mechanische Anhebbewegung des linken Mittelbeins resultiert in einem lokalen Widerstandsreflex (Muskelaktivität des linken Depressors DL) und in einer synchron gesteigerten Aktivität des Neurons. Diese scheint außer von der Dauer der Auslenkung auch von der Amplitude des Anhebens abhängig zu sein (Abb. 8, B; Veränderung der Spikefrequenz im weiteren Verlauf der Auslenkbewegung). Bei Reizung des Tarsus vom ipsilateralen Mittelbein erscheint nur eine schwach gesteigerte Aktivität des Neurons (Abb. 9, C). In einem Fall erscheint eine sehr kurze, phasische Erregung (knapp $70 \mathrm{~Hz}$ ). Während dieser Reizserie erfolgt aber kein dauerhafter Anstieg der Frequenz nach den Folgereizungen. Passives Anheben dieses Beins (Abb. 9, D) führt zu einer synchron verminderten Neuronaktivität, die ebenfalls von Dauer und Ausmaß der Anhebbewegung abhängig zu sein scheint 
(zunehmende, nicht vollständige Hemmung des Neurons). Eine einmal aufgetretene aktive Bewegung des rechten Mittelbeins (nicht gereizt) führte zu einer vollständigen Hemmung des Neurons für die gesamte Dauer der Aktivität des linken Depressormuskels (Abb. 10, E).

Kurzdiskussion:

Aufgrund der physiologischen Eigenschaften und den im Mesothorakalganglion zahlreich vorhandenen varikosen Fasern, die mögliche Outputregionen kennzeichnen und die nebeneinander mit dendritischen Fasern (Input) vorkommen, erscheint eine spezifische Funktion im Kontext der bilateralen Koordination zumindest auf indirektem Wege denkbar. Dafür spricht die zeitlich strenge Korrelation der Neuronaktivität mit der Muskelaktivität der beiden Depressoren und die Tatsache, dass Erregung des Neurons während bilateraler Reflexe unidirektional auftritt (linkes Mittelbein gereizt, DL aktiv), es in der entgegengesetzten Richtung (rechtes Mittelbein gereizt, DR aktiv) aber offensichtlich zu einer Hemmung des Neurons kommt. Solch eine Hemmung erscheint im Kontext einer bilateralen Koordination sinnvoll, denn, wenn das Neuron einen für Motoneurone des linken Depressors erregenden Einfluss hat, so wäre dessen Aktivität störend, wenn zum Beispiel durch einen entgegen gerichteten Reflex gerade das linke Bein angehoben wird (linker Levator des Trochanters aktiv). Eine direkte Verschaltung auf Motoneurone des linken Depressormuskels ist jedoch fraglich. Keines der Verzweigungsfelder des Neurons reicht extrem weit nach lateral, so dass sie, wenn, dann nur knapp in die dorsalen Neuropile (pLAC, aLAC) hineinreichen dürften. Auch ist eine deutliche Trennung von Input- und Outputregionen bei diesem Neuron nicht der Fall. De- oder Hyperpolarisation hatte zwar die für das Neuron zu erwartenden Effekte, hatte aber keinen erkennbaren direkten Einfluss auf die Muskelaktivität, was zumindest nicht für eine erregende (direkte oder indirekte) Verschaltung des Neurons auf Motoneurone spricht.

Aufgrund der morphologischen Gegebenheiten ist eine im Kontext von Extremitätenbewegungen intersegmental koordinierende Funktion des Neurons zu erwarten. Ob und gegebenenfalls wo das Interneuron Input- und/oder Outputregionen im Pro- und Metathorakalganglion besitzt, ist aufgrund unvollständiger Färbung aber nicht bekannt. Reizung der Vorderbeine und von Haaren am 
Thorax/Abdomen führte bei diesem Präparat zu keinen erkennbaren Reaktionen. Aufgrund der generell eher nur schwach erkennbaren Reflexe wurden beide Mittelbeine zusätzlich auch mechanisch ausgelenkt (angehoben), auch auf die Gefahr hin, dass eine vermeintlich daraus resultierende Veränderung der Neuronaktivität tatsächlich auf ein Ableitartefakt durch mechanische Reizung des Neurons mit der Glasmikroelektrode zurückzuführen ist. Da aber Anheben des linken Beins zu Erregung des Neurons, während Anheben rechts zu seiner Hemmung führt, und auch bei einer aktiven Bewegungssequenz des rechten Beins (keine Reizung) vollständige Hemmung auftritt, kann diesen Ergebnissen eine gewisse Aussagekraft beigemessen werden.

Zusammengefasst lässt sich sagen, dass dieses Neuron eine im Kontext von Bewegungen der Extremitäten koordinierende Funktion zu haben scheint, dass eine bilaterale Koordination, wenn, dann eher auf indirektem Wege erfolgt, entweder über ein anderes vor- oder ein nachgeschaltetes (lokales) Interneuron. Auch scheint keine klare Trennung zwischen rein bilateraler oder intersegmentaler Koordination vorzuliegen, wie dies häufig bei kommissuralen Interneuronen mit Extremitätenkoordinierenden Eigenschaften der Fall zu sein scheint. Eine lediglich sensorischintegrierende Funktion des Neurons ist nicht zu erwarten, da es während aktiver Bewegung (vor allem bei Aktivität des rechten Depressormuskels) gehemmt wird.

\subsubsection{Bilateral verzweigtes kommissurales Typ CIN-I Interneuron CIN-I(a)c1}

Morphologie:

Mesothorakalganglion:

Die Abbildungen 11-12 zeigen das dorsal im kontralateralen (rechten) Konnektiv des Mesothorakalganglions aufsteigende Interneuron CIN-I(a)c1 mit bilateralem Verzweigungsmuster. Das Soma ist ventral im posterioren Teil des linken Hemiganglions lokalisiert, auf der Höhe relativ zur äußeren Randzone der ipsilateralen sich longitudinal gegenüber liegenden Konnektive. Es besitzt einen Durchmesser von etwa $25 \mu \mathrm{m}$. Der primäre Neurit zieht bogenförmig zur Mitte des ipsilateralen Hemiganglions und steigt dabei sehr weit dorsal auf. Eine erste kleinere Nebenverzweigung verläuft zurück nach posterior, wo sie sich in der posterioren 


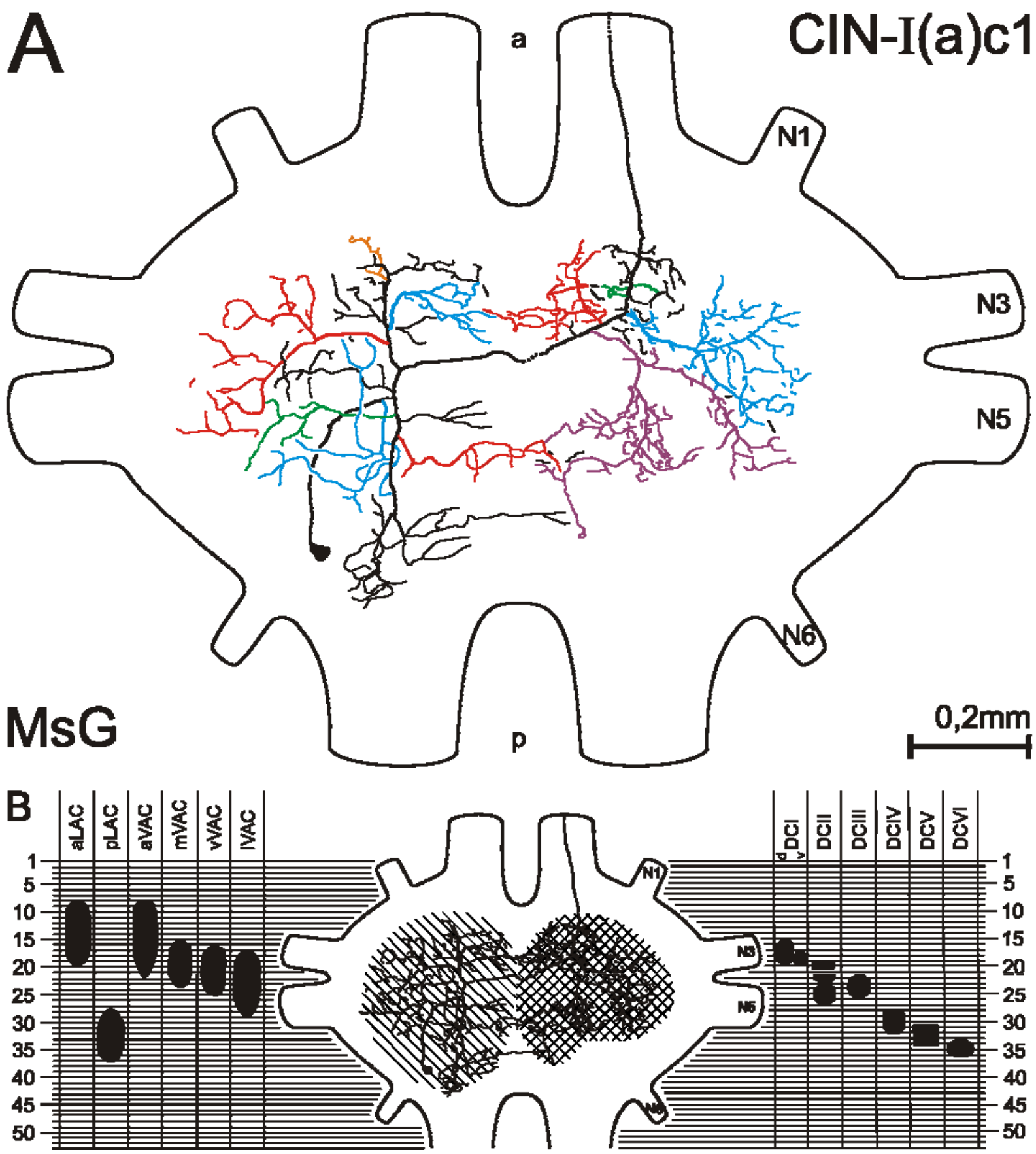

Abb. 11: Intersegmentales Interneuron CIN-I(a)c1 mit bilateralem Verzweigungsmuster (Typ I). A: Gezeichnete 2D-Projektion, Mesothorakalganglion. Dorsal im kontralateralen (rechten) Konnektiv aufsteigendes Axon. Die meisten Verzweigungen erstrecken sich vorwiegend dorsal-lateral über einen weiten Bereich in beiden Hemiganglien. Vor allem im ipsilateralen Hemiganglion reichen sie bis an die dorsalen Neuropile (pLAC, aLAC) heran. Eine ipsilaterale Nebenverzweigung erstreckt sich posterior bis in den intermediären Bereich. Der primäre Neurit passiert die dorsale Kommissur DCII einmal, aber es gibt mehrere Verzweigungen, die ebenfalls über dorsale Kommissuren (DCI und DCIV-DCVI) in das jeweils gegenüberliegende Hemiganglion ziehen und mit lokalen Verzweigungen überlappen. Das Soma $(\varnothing=25 \mu \mathrm{m})$ liegt lateral und posterior-ventral. B: Übersicht desselben Neurons und Vergleich mit der relativen Lage wichtiger morphologischer Strukturen (aus: PFLÜGER et al. 1988; leicht modifiziert). Abkürzungen: MsG: Mesothorakalganglion; a: anterior; p: posterior; N1-N6: Nerv16; Schraffur in B: Bereich, in welchem varikose Fasern (potentiell lokale Projektionsbereiche) nebeneinander mit mehr dendritischen Fasern vorkommen; Kreuzschraffur in B: Vorwiegend Vorkommen von varikosen Fasern; Abkürzungen in B Siehe Tabelle der verwendeten Abkürzungen. 


\section{CIN-I(a)c1}

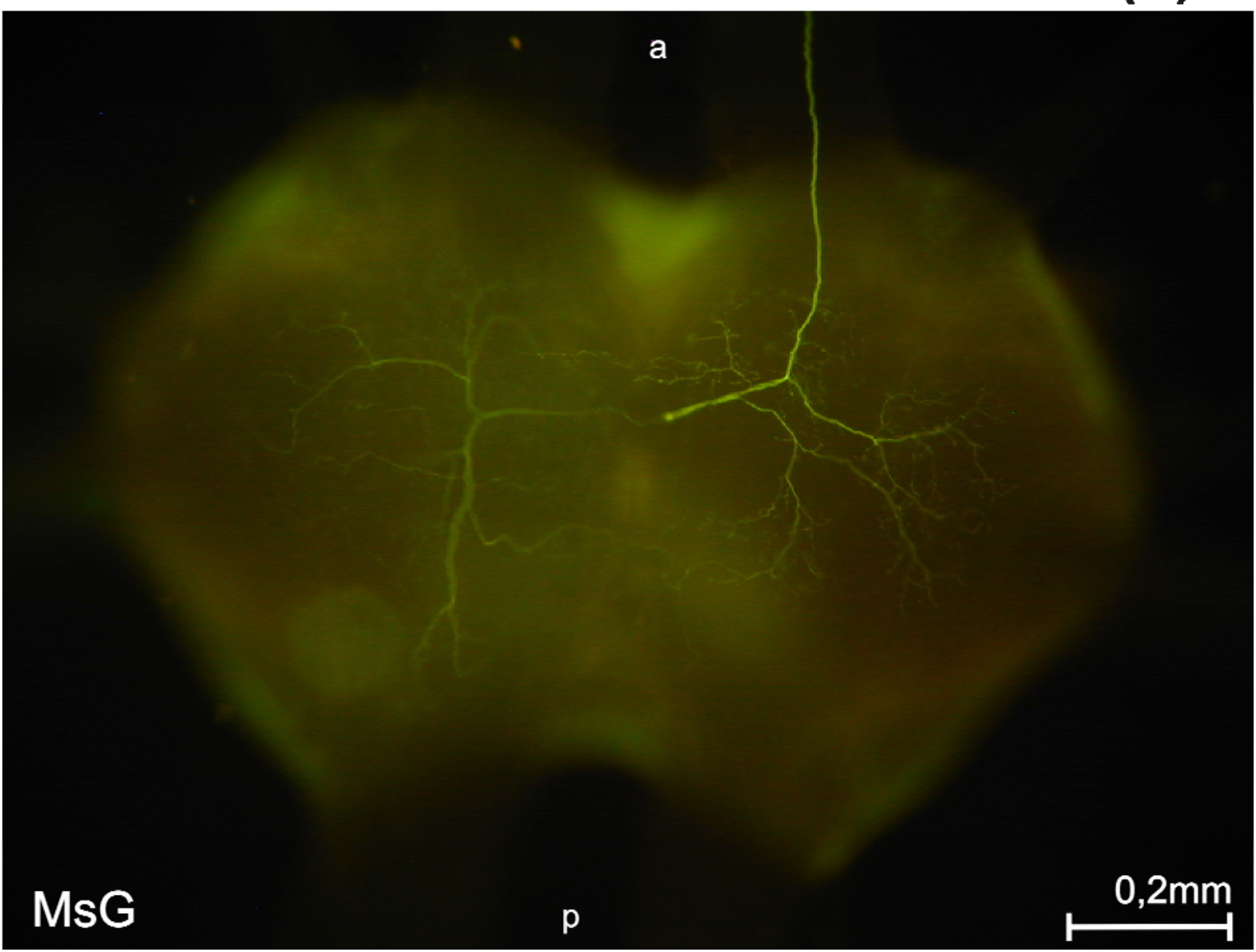

Abb. 12: Originalfotografie des Neurons CIN-I(a)c1, Mesothorakalganglion. Die Fokalebene liegt sehr weit dorsal auf dem Niveau der bilateralen Hauptverzweigungsbereiche, die sich in beiden Hemiganglien relativ weit nach lateral erstrecken. Auch das sehr weit dorsal im kontralateralen (rechten) Konnektiv aufsteigende Axon ist fokal getroffen. Der Durchtritt des primären Neuriten durch die dorsale Kommissur DCII ist etwas schwach und unscharf zu erkennen. In den Verzweigungsarealen beider Hemiganglien, vor allem jedoch im kontralateralen Hemiganglion tragen zahlreiche Fasern lokale kleine Verdickungen (Varikositäten). Diese Strukturen kennzeichnen potentielle Projektionsbereiche des Neurons (Output-Strukturen). Das ventral gelegene Soma ist nur schwach und sehr diffus in der Tiefe des ipsilateralen (linken) Hemiganglions zu erkennen.

Abkürzungen: MsG: Mesothorakalganglion; a: anterior; p: posterior.

Hälfte des ipsilateralen Hemiganglions hauptsächlich dorsal, zum Teil aber auch bis in den intermediären Bereich hinein weiterverzweigt. Der primäre Neurit teilt sich ein kurzes Stück weiter in zwei Äste auf, einen posterior und einen anterior gelegenen, die zusammen genommen einen weiten Bereich des ipsilateralen Hemiganglions bedecken und sich ausschließlich äußerst weit dorsal verzweigen.

Der posterior gelegene Ast (erste Hauptverzweigung) erstreckt sich nicht so weit nach lateral wie der anterior gelegene. Er besitzt zwei Ausläufer, die über die hinteren dorsalen Kommissuren (DCIV-VI) bis in das kontralaterale Hemiganglion 
hinein ziehen und dort knapp mit lokalen Verzweigungen überlappen.

Der anterior gelegene Ast (zweite Hauptverzweigung) erstreckt sich weiter nach lateral und dürfte die dorsalen Neuropile ( $p L A C, a L A C$ ) erreichen. Er hat aber keine Verzweigungen, die eine Kommissur passieren und somit in das kontralaterale Hemiganglion hineinreichen könnten.

Etwa auf mittlerer Höhe der Ganglion-Querachse beginnt der kommissurale Fortsatz des primären Neuriten, der nach Passieren der dorsalen Kommissur DCIl ein wenig nach anterior zieht, etwa bis zur gedachten Vertikalen durch die Mitte des kontralateralen Konnektivs, von wo aus das Axon median im kontralateralen Konnektiv dorsal aufsteigt. Im kontralateralen Hemiganglion trägt der Neurit drei weitere Hauptverzweigungen (3 bis 5). Die dritte trennt sich posterior vom Neuriten $a b$, zieht noch ein Stück nach lateral und teilt sich dann in einen mehr dorsal-lateral sowie einen weiter nach posterior verlaufenden und dorsal-median verzweigenden Teil. Letzterer zieht in Richtung der Mittellinie, wo er knapp mit kommissuralen Verzweigungen des ipsilateralen Hemiganglions überlappt, ohne selbst eine Kommissur zu passieren. Der dorsal-lateral verzweigende Ausläufer könnte knapp bis an die dorsalen Neuropile (pLAC, aLAC) heranreichen.

Die vierte Hauptverzweigung trennt sich anterior vom primären Neuriten, verläuft anterior-median und dorsal und besitzt auch Ausläufer, die über die vorderen dorsalen Kommissuren DCl-II bis in das ipsilaterale Hemiganglion an Verzweigungen des anterioren Astes knapp heranreichen.

Die fünfte Hauptverzweigung erstreckt sich wiederum dorsal über einen größeren Bereich der lateralen Hälfte des Hemiganglions. Auch hier könnten Verzweigungen an die dorsalen Neuropile (pLAC, aLAC) heranreichen. Das aufsteigende Axon selbst besitzt einige kleinere Kollaterale, die manchmal knapp an Verzweigungen der Hauptausläufer heranreichen, sonst aber eher auf einen kleineren lokalen Bereich beschränkt sind.

Alle Verzweigungen in beiden Hemiganglien weisen zahlreiche Varikositäten auf. An den Fasern des kontralateralen Hemiganglions, die insgesamt stärker durch den Fluoreszenzfarbstoff gefärbt sind, erscheinen sie deutlicher erkennbar und noch zahlreicher. Die Verzweigungen wirken hier auch weniger filigran als die auf ipsilateraler Seite. 


\section{Prothorakalganglion:}

Die neuronale Verzweigungsstruktur im Prothorakalganglion ist aufgrund unvollständiger Färbung nicht bekannt.

Physiologie:

Das phasisch-tonische Neuron CIN-I(a)c1 hat einen sehr unregelmäßigen Tonus, der über den gesamten Aufzeichnungszeitraum zwischen $20 \mathrm{~Hz}$ bis über $100 \mathrm{~Hz}$ schwankt. Die Heuschrecke war zu diesem Zeitpunkt in der Bewegungsfähigkeit etwas eingeschränkt, so dass sich Reflexe nicht immer zuverlässig und unmittelbar auslösen ließen. Deshalb ist die Reizindikation hier nicht als zeitgenau zugeordneter Einzelreiz zu verstehen, sondern als Beginn einer kontinuierlichen Reizung (Abb. 13, A; gestrichelte Linie in der ST-Spur) bis zur Reflexauslösung (gemessene Aktivität des rechten Depressormuskels DR). Das Neuron reagiert auf Reizung des kontralateralen (rechten) Vorderbeins und beider Mittelbeine, wenn auch nur mit schwachem Frequenzanstieg. Manchmal erscheint eine leicht erhöhte Spikefrequenz bis etwa $130 \mathrm{~Hz}$ mehr oder weniger synchron zur Depressoraktivität, aber nicht in strenger Korrelation und auch nicht immer. Die Instantanfrequenz übersteigt die Spontanfrequenz von knapp $60 \mathrm{~Hz}$ immer nur kurzzeitig und nicht besonders stark.

Manchmal traten beim Präparat während der Aufzeichnungen spontane leichte Zuckbewegungen beider Mittelbeine (synchrones Zucken, keine Laufbewegungen) auf, die an der Aktivität des kontralateralen (rechten) Depressormuskels zu erkennen sind (Abb. 13, B). Das Neuron zeigt hierbei ein interessantes und streng zur Muskelaktivität korreliertes Verhalten: Es erscheinen rhythmische, zur Muskelaktivität korrelierte Bursts und dabei treten Spikes von deutlich unterschiedlicher Amplitude auf. Die Spikes mit der kleineren Amplitude (im Vergleich halb so groß oder geringer) erscheinen fast ausschließlich nur innerhalb dieser Bursts und sind streng von den Spikes abgesetzt, die auch während der Spontanaktivität des Neurons auftreten. Darüber hinaus sitzen sie einem elektrotonischen Potential auf (vermutlich in der näheren Umgebung erzeugtes EPSP). Die Bursts lassen sich somit in zwei Phasen aufteilen: Jeder Burst beginnt mit einer ersten Phase (Dauer etwa 50-100ms), in der Spikes mit großer Amplitude auftreten und einer zweiten (Dauer etwa 50ms), in der nur Spikes mit kleiner Amplitude erscheinen (Abb. 13, C). In der Regel etwas verzögert zu Phase 1 setzt die Muskelaktivität ein, die dann bis zum Ende dieser 


\section{CIN-I(a)c1}
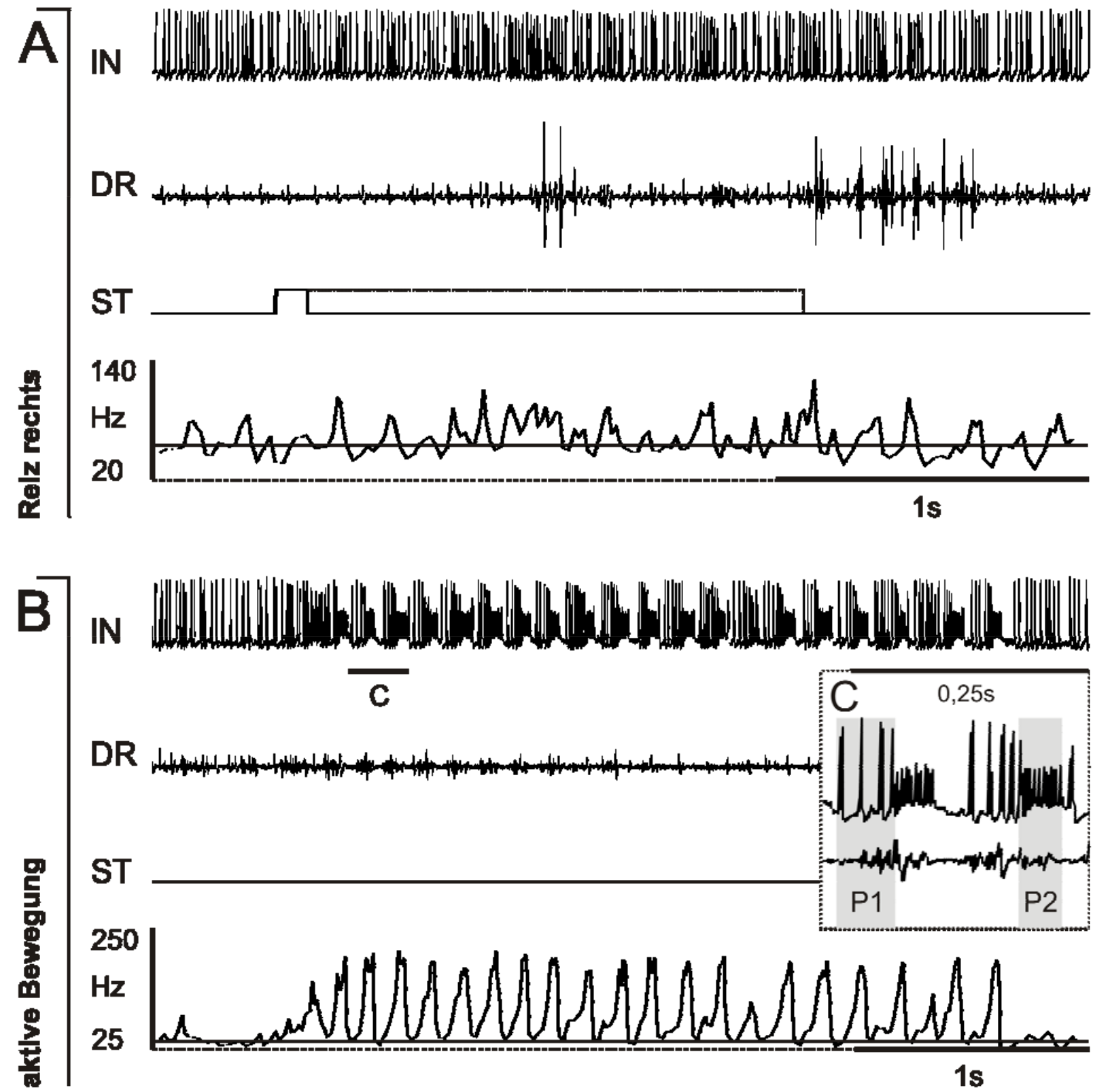

Abb. 13: Aktivität, Instantan- und Spontanfrequenzen des Neurons CIN-I(a)c1 und Muskelaktivitäten bei kontralateraler Reizung (rechtes Mittelbein) sowie aktiver Bewegung (rhythmisches Zucken beider Mittelbeine, Ableitung vom linken Depressormuskel fehlgeschlagen). Das Neuron (IN) ist bei $20-60 \mathrm{~Hz}$ tonisch aktiv. A: Dorsaler taktiler Tarsus-Reiz am rechten Mittelbein (kontinuierliche Reizung bis zum Reizerfolg, gestrichelte Linie in der ST-Spur). Es erscheint eine leicht erhöhte Neuronaktivität (bis $130 \mathrm{~Hz}$ ), in nicht strenger Korrelation zur Muskelaktivität (rechter Depressormuskel, DR). Sie setzt kurz vorher ein und dauert während der Muskelaktivität zum Teil noch etwas an, ist aber vor deren Abklingen beendet. B: Aktive Bewegungssequenz: Es erscheinen zeitlich streng zur Muskelaktivität synchron korrelierte Neuronbursts (bei $100-250 \mathrm{~Hz}$ ), mit folgender Besonderheit: Jeder Burst besteht aus einer Phase 1, in der Spikes mit Ausgangs-Amplitude erscheinen, sowie einer Phase 2, in der deutlich abgegrenzt Spikes mit deutlich geringerer Amplitude erscheinen. Dabei beginnt zunächst Phase 1, zeitlich etwas verzögert setzt die Muskelaktivität ein, die meist unmittelbar oder kurz nach dem Übergang von Phase 1 zu Phase 2 endet. C: Vergrößerter Ausschnitt der IN- und DR-Spur aus B, der die zwei Phasen zeigt. Die Spikes mit kleiner Amplitude (Phase P2) sitzen einem EPSP auf. Abkürzungen: IN: Aktivität des Neurons; DR: Depressor trochanteris, rechtes Mittelbein; DL: Depressor tr., linkes Mittelbein; ST: dorsal-taktiler Tarsusreiz. 
ersten Phase der Neuron-Bursts synchron aktiv ist. In den meisten Fällen endet sie unmittelbar am Übergang von der ersten zur zweiten Phase, manchmal dauert sie während Phase 2 auch minimal noch etwas an, endet aber immer bevor Phase 2 beendet ist. Während der Bursts kann die Instantanfrequenz durchschnittlich von $100 \mathrm{~Hz}$ bis knapp auf $250 \mathrm{~Hz}$ ansteigen. Vor allem während der zweiten Phase werden die höchsten Frequenzen erreicht. Zwischen den Bursts erscheinen während einer Pause von 20-30ms in der Regel keine Spikes, die Instantanfrequenz fällt dann meist genau auf das Niveau der Spontanfrequenz zurück bevor der nächste Burst beginnt.

Kurzdiskussion:

Ob dem Neuron CIN-I(a)c1 eine bilateral koordinierende Funktion zukommt, lässt sich nicht eindeutig sagen. Dafür sind zu wenige spezifische Daten vorhanden. Nur eine der beiden Ableitungen von den Depressormuskeln war erfolgreich, wobei das Tier zum Zeitpunkt der Aufzeichnungen etwas bewegungsreduziert war. Die ausgelösten Reflexe waren manchmal nur schwach sichtbar und hatten eher einen geringen Effekt auf die Neuronaktivität und eine klare Korrelation zur Muskelaktivität war nicht erkennbar. Die strenge synchrone Korrelation der Neuronbursts mit der Aktivität des kontralateralen Depressormuskels während einer spontanen Bewegungssequenz (ohne Reizung aufgetretene rhythmisch-synchrone Muskelzuckungen beider Mittelbeine) legt jedoch eine allgemein die Extremitäten koordinierende Funktion nahe. Die Morphologie zeigt nämlich Verzweigungen des Neurons dorsal in beiden Hemiganglien bis weit nach lateral in den Bereich der dorsalen Neuropile (pLAC, aLAC) hinein. Das Neuron könnte somit einerseits sensorischen Input von in diese lateralen Neuropile projizierenden Mechanorezeptoren erhalten (campaniforme Sensillen, Chordotonalorgane, GelenkstellungsRezeptoren) und andererseits direkt auf Motoneurone verschalten, zumal viele Fasern des Neurons in beiden Hemiganglien Output-Charakter (zahlreiche Varikositäten) haben: So ist eine bilateral koordinierende Funktion theoretisch denkbar. Dafür spricht auch die Tatsache, dass einige Fasern aus Verzweigungen beider Hemiganglien jeweils über eine der dorsalen Kommissuren mit Verzweigungen der gegenüberliegenden Seite kommunizieren (rekurrente Fasern) und somit zusätzlich bilaterale Information ausgetauscht werden könnte. Eine 
ausschließlich bilateral koordinierende Funktion ist aber nicht zu erwarten, da es sich um ein großes intersegmentales Interneuron mit einem im kontralateralen (rechten) Konnektiv ins Prothorakalganglion aufsteigenden Axon handelt und da das Neuron auch auf Reizung des kontralateralen Vorderbeins reagiert. Genaueres über die Art der intersegmentalen Koordination kann aber nicht ausgesagt werden, zum einen wegen der genannten Schwierigkeiten, durch Reizung sichtbar Reflexe auszulösen, zum anderen auch deswegen, weil die Morphologie des Neurons im Prothorakalganglion aufgrund unvollständiger Färbung nicht bekannt ist.

Merkwürdig und etwas schwer zu interpretieren ist das Auftreten von Spikes unterschiedlicher Amplitude in Bursts ein und desselben Neurons. Es ist nicht auszuschließen, dass trotz einer stabilen intrazellulären Ableitung auch die Aktivität eines anderen Neurons theoretisch mitregistriert werden kann. Aber jene Spikes mit kleinerer Amplitude erscheinen fast ausschließlich nur in den neuronalen Bursts, die streng mit der Muskelaktivität korreliert sind (nach Reizung oder auch bei aktiver Bewegung, nur ganz vereinzelt aber während der Spontanaktivität des Neurons, ohne parallel auftretende Muskelaktivitäten). Dass der Effekt über präsynaptische Hemmung durch ein anderes Interneuron zustande kommt ist unwahrscheinlich, da keine allmählich zunehmende Abschwächung der Spike-Amplitude zu beobachten ist. Die Spikes mit kleinerer Amplitude sind klar von solchen mit Ausgangsamplitude abgegrenzt und untereinander nahezu konstant. Große intersegmentale Interneurone verfügen oftmals über mehrere Spike-generierende Zonen (HUSTERT 1985). Es wäre also denkbar, dass der beobachtete Effekt auf das Vorhandensein von mehreren solcher Triggerzonen zurückzuführen ist. An einer konstanten Ableitstelle an der Membran sollten andererseits die physikalischen Vorgänge bei der Fortleitung eines Aktionspotentials (Kaskade der Ladungsverschiebungen) und damit die Amplitude der durchlaufenden Aktionspotentiale konstant sein (wenn es keinen nennenswerten modulierenden Einfluss auf das Ruhepotential gibt), unabhängig vom Ort ihrer Entstehung. Im Falle von großen intersegmentalen Interneuronen mit komplexem Verzweigungsmuster und mehreren Triggerzonen für Spikes ist nicht anzunehmen, dass sich Aktionspotentiale, die weiter entfernt generiert und über das Axon mit einem dickeren Durchmesser in den Projektionsbereich geleitet werden, auch wieder über feinste und weit entfernte dendritische Nebenverzweigungen in einem anderen Ganglion ausbreiten. Überhaupt müssten Aktionspotentiale, die an verschiedenen Triggerzonen ausgelöst und sich zum Teil über die gleichen Pfade 
ausbreiten, theoretisch früher oder später zwangsläufig miteinander kollidieren, was im Sinne der Informationsübertragung nachteilig wäre, da sich die Spikes gegenseitig auslöschen müssten. Ohnehin ist eine solche Spike-Kollision im intakten Tier in der Forschung bislang noch nicht nachgewiesen worden. Möglicherweise handelt es sich bei den beobachteten Spikes mit kleinerer Amplitude um einen sich in Verzweigungen mit dünnerem Durchmesser passiv und elektrotonisch ausbreitenden Effekt, der auf weiter entfernt durch das Axon geleitete Aktionspotentiale zurückzuführen ist. Dagegen spricht allerdings wieder die Tatsache, dass die innerhalb der Phase 2 auftretenden Spikes mit kleiner Amplitude einem dauerhaft depolarisierendem DC-Potential aufsitzen (für die Dauer der Phase 2). Dabei kann es sich nur um ein einzelnes oder mehrere aufsummierte postsynaptische Potentiale handeln, welche in der näheren Umgebung des Ableitortes ausgelöst worden sein müssen, da sich deren Amplitude bei der elektrotonischen Fortleitung schnell abschwächt (abhängig von der Membran-Längskonstante). Dennoch ist dies ein in großen intersegmentalen und kommissuralen Interneuronen manchmal auftretendes Phänomen. Eine vergleichbare Situation zeigt sich beispielsweise bei einem weiteren bilateral verzweigenden und hier vorgestellten Neuron, dem Neuron CIN-I(d)c2), das ebenfalls Eigenschaften einer die Extremitäten koordinierenden Funktion zu haben scheint. Aufgrund der strengen Korrelation dieses neuronalen Verhaltens zu Muskelaktivitäten während der Bewegungen der Extremitäten spielt dieser Effekt möglicherweise sogar eine wichtige Rolle im Kontext von intersegmentaler und/oder bilateraler Koordination. Anhand der vorliegenden morphologischen und physiologischen Daten kann dazu jedoch nichts Genaueres ausgesagt werden.

\subsubsection{Bilateral verzweigtes kommissurales Typ CIN-I Interneuron CIN-I(d)c1}

Morphologie:

Mesothorakalganglion:

Das intersegmentale Interneuron $\mathrm{CIN}-\mathrm{I}(\mathrm{d}) \mathrm{c} 1$ ist durch bilaterale Verzweigungsbereiche gekennzeichnet und steigt im kontralateralen (linken) Konnektiv dorsal ab (Abb. 14-15). Auffällig ist das klar getrennte Vorkommen von varikosen Fasern (potentieller lokaler Projektionsbereich) im kontralateralen Hemiganglion, gegenüber 
Fasern mit dendritischem Charakter im ipsilateralen Hemiganglion. Der Neurit durchquert eine der mittleren dorsalen Kommissuren, wahrscheinlich DCIII, da er sich auf intermediärem Niveau und nicht dorsal befindet.

Das Soma hat einen Durchmesser von etwa $20 \mu \mathrm{m}$ und liegt ventral und anteriorlateral im rechten Hemiganglion auf der Höhe der gedachten longitudinalen Verbindungslinie entlang des lateralen Randbereiches der ipsilateral gegenüberliegenden Konnektive. Der primäre Neurit zieht zunächst für ca. $25 \mu \mathrm{m}$ weiter nach lateral, verläuft dann mit einem Knick nach posterior und zieht bis auf Höhe der mittleren Ganglion-Querachse. Dabei steigt er zunehmend dorsal auf. Entlang dieses Stückes treten nur wenige kleinere und lokal beschränkte Verzweigungen im ventralen bis intermediären Bereich auf.

Auf der Höhe zwischen Nerv 3 und Nerv 5 entspringt die erste Hauptverzweigung und zieht zurück nach anterior, wo sie oberhalb des Somas im intermediären Bereich weiter verzweigt. Im anschließenden Stück bildet der primäre Neurit eine verdickte Struktur, die in Richtung der Mittellinie zieht. Alle vier weiteren Hauptverzweigungen ziehen von diesem verdickten Prozess in die Peripherie und verteilen sich dabei mehr oder weniger sternförmig. Die zweite Hauptverzweigung zieht weiter in den lateralen Bereich des Hemiganglions und teilt sich in vier Ausläufer auf, die intermediär bis äußerst weit dorsal-lateral verzweigen. Die Verzweigungen der beiden peripheren Ausläufer reichen dabei in das posteriore (pLAC) und möglicherweise auch in das anteriore dorsale Neuropil (aLAC) hinein.

Die dritte Hauptverzweigung zieht auf der Höhe des Konnektivs nach posterior und steigt vom intermediären Niveau bis nach dorsal auf. Das entspricht in etwa dem Bereich, in welchem die verschiedenen dorsalen Trakte (DIT, DMT, LDT) lokalisiert sind. Die vierte und fünfte Hauptverzweigung, die jeweils ein kurzes Stück weiter weg vom Neuriten abzweigen (etwa $15 \mu \mathrm{m}$ ), liegen sich direkt gegenüber.

Die vierte zieht ebenfalls nach posterior, gabelt sich dann allerdings in zwei Bereiche auf, die beide sehr weit dorsal verzweigen und zum größten Teil über den Verzweigungen des dritten Hauptausläufers liegen und sich dabei zunehmend voneinander entfernen. Die innere größere Verzweigung verläuft in Richtung der Mittellinie in einem Bereich, in welchem auch in etwa der mediane dorsale Trakt (MDT) verläuft. Die fünfte Hauptverzweigung erstreckt sich in anterior-medianer Richtung ebenfalls sehr weit dorsal. Morphologische relevante Strukturen in diesem Bereich sind wiederum die dorsalen Trakte (MDT, DIT und eventuell DMT). 

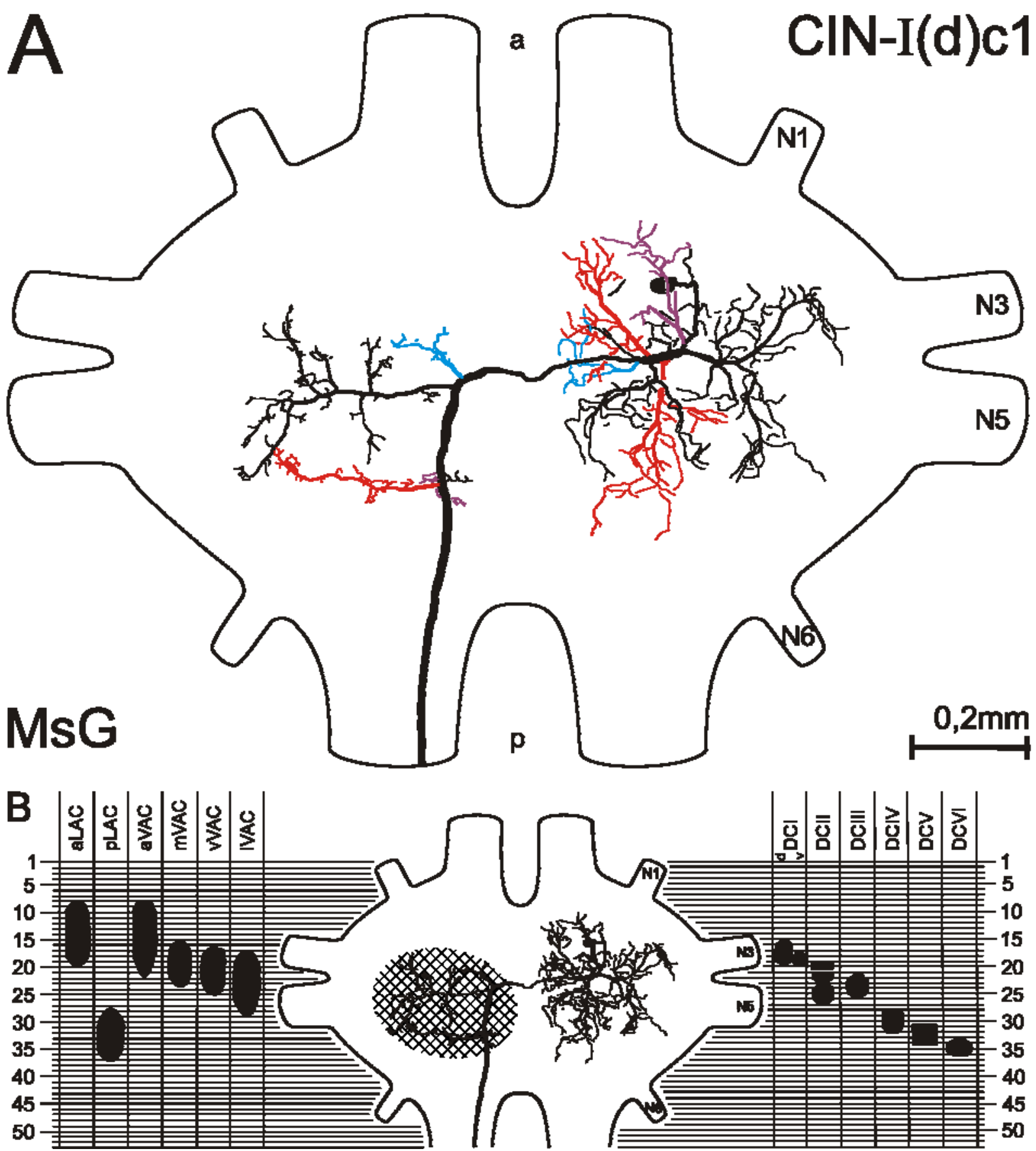

Abb. 14: Intersegmentales Interneuron CIN-I(d)c1 mit bilateralem Verzweigungsmuster (Typ I). A: Gezeichnete 2D-Projektion, Mesothorakalganglion. Dorsal im kontralateralen (linken) Konnektiv absteigendes Axon. Alle Hauptverzweigungen des ipsilateralen Hemiganglions verzweigen überwiegend dorsal-median bis dorsal-lateral, steigen dabei zum Teil aus dem intermediären Bereich auf. Einige der lateralen Ausläufer erreichen das anteriore (aLAC) und auch das posteriore dorsale Neuropil (pLAC). Eine ipsilaterale Nebenverzweigung erstreckt sich posterior bis in den intermediären Bereich. Der primäre Neurit passiert wahrscheinlich die dorsale Kommissur DCIII (intermediäre bis dorsale Höhe). Die Färbung zeigte keine kommissuralen Nebenverzweigungen. Das absteigende Axon trägt große Kollaterale, die ebenfalls dorsal-median bis dorsal-lateral verzweigen und zum Teil knapp an die dorsalen Neuropile heranreichen. Alle Kollaterale tragen deutlich erkennbare Varikositäten. Das Soma $(\varnothing=20 \mu \mathrm{m})$ liegt ventral und anterior-lateral. B: Übersicht desselben Neurons und Vergleich mit der relativen Lage wichtiger morphologischer Strukturen (aus: PFLÜGER et al. 1988; leicht modifiziert).

Abkürzungen: MsG: Mesothorakalganglion; a: anterior; p: posterior; N1-N6: Nerv1-6; Kreuzschraffur in B: Bereich, in welchem nahezu ausschließlich varikose Fasern (potentiell lokale Projektionsbereiche) vorkommen; Abkürzungen in B Siehe Tabelle der verwendeten Abkürzungen. 

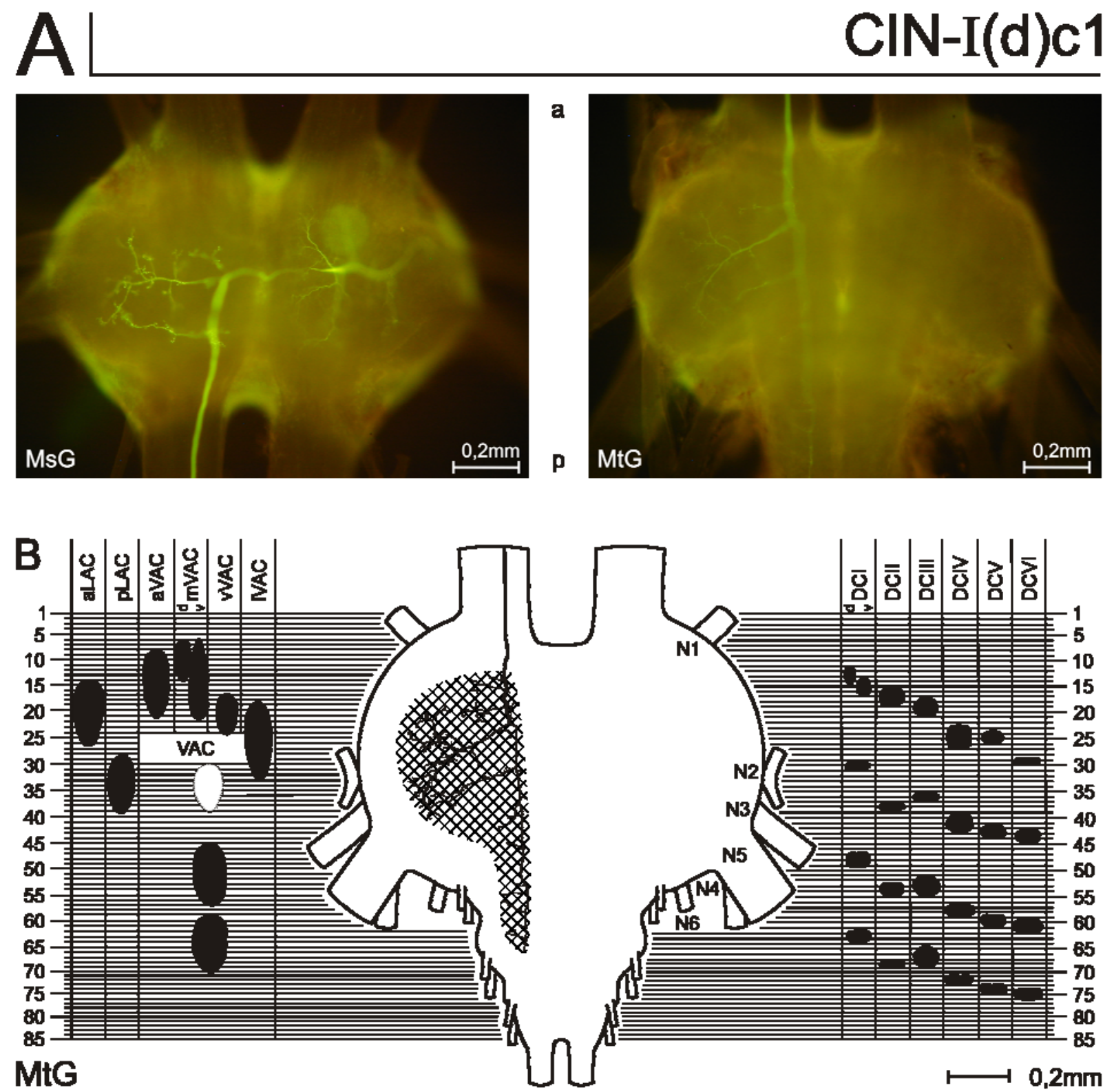

Abb. 15: A: Originalfotografien des Neurons CIN-I(d)c1. MsG: Mesothorakalganglion. Dorsale Aufsicht auf die bilateral angeordneten dorsalen Hauptverzweigungsbereiche. Das Axon steigt dorsal im kontralateralen (linken) Konnektiv ab. Alle Verzweigungen der kontralateralen (linken) Seite tragen ausgeprägte Varikositäten, die sie als potentielle Projektionsbereiche kennzeichnen. Die Fasern des ipsilateralen Hemiganglions dagegen sind fast ausschließlich von dendritischem Charakter. Der primäre Neurit durchquert wahrscheinlich die dorsale Kommissur DCIII, die in diesem Bereich ventral von der dorsalen Kommissur DCll gelegen ist. Er erscheint daher etwas unscharf abgebildet. Das ventral gelegene Soma ist schwach und diffus im ipsilateralen (rechten) Hemiganglion erkennbar. MtG: Metathorakalganglion. Der Projektionsbereich des absteigenden Axons beschränkt sich hier ausschließlich auf die kontralaterale Hälfte, erstreckt sich dort jedoch über einen weiten Bereich bis zu den angelagerten Abdominalganglien. Alle Verzweigungen tragen Varikositäten, ziehen auf dorsaler Höhe zunächst nach lateral und verlaufen dort von dorsal bis in den intermediären Bereich. Das Hauptverzweigungsareal liegt im Bereich der lateralen Neuropile ( $\mathrm{pLAC}$, aLAC) des Metathorakalganglions. B: Gezeichnete 2D-Projektion der metathorakalen Strukturen des Neurons und Vergleich mit der relativen Lage wichtiger morphologischer Strukturen (aus: PFLÜGER et al. 1988; leicht modifiziert).

Abkürzungen: MsG: Mesothorakalganglion; MtG: Metathorakalganglion; a: anterior; p: posterior; N1N6: Nerv1-6; Kreuzschraffur in B: Bereich, in welchem nahezu ausschließlich varikose Fasern (vermutlich Projektionsbereich) vorkommen; Abkürzungen in B Siehe Tabelle der verwendeten Abkürzungen. 
Nach dem Abzweigen der fünften Hauptverzweigung verjüngt sich der primäre Neurit wieder, bevor er schließlich die dorsale Kommissur (wahrscheinlich DCIII) auf intermediärer Höhe passiert. Das im kontralateralen Konnektiv absteigende Axon verläuft sehr weit dorsal. Es trägt vier Kollaterale, eine erste kleinere, die nach anterior-lateral zieht und dorsal verzweigt, und darauf folgend zwei große, die in einem weiteren dorsal-lateralen Bereich verzweigen und lateral bis in den intermediären Bereich hinunterziehen. Beide, vor allem aber die zweite Kollaterale, reichen knapp bis an die lateralen Neuropile (pLAC, aLAC) heran. Die vierte Kollaterale liegt der Dritten direkt gegenüber und ist auf einen kleinen dorsal gelegenen Bereich beschränkt. Alle vier Kollateralen tragen kräftigere und weniger komplex verzweigte Fasern und besitzen zahlreiche Varikositäten, die deutlich erkennbar sind.

\section{Metathorakalganglion:}

Das im kontralateralen (linken) Konnektiv absteigende Axon des Neurons CIN-I(d)c1 gelangt sehr weit dorsal in das Metathorakalganglion und projiziert in ein weites Feld, das neben dem Metathorakalganglion zumindest auch noch das erste Neuromer der drei angelagerten Abdominalganglien umfasst (Abb. 15, B). Möglicherweise projiziert das Neuron auch noch weiter in die folgenden Abdominalganglien. Aber das ist aufgrund der hier nicht vollständigen Färbung nicht mehr genau zu erfassen. Im gesamten erkennbaren Feld des Metathorakalganglions bleibt der Projektionsbereich auf das kontralaterale Hemiganglion beschränkt. Zahlreiche Ausläufer ziehen nach dorsal-lateral, jeweils in Richtung abgehender Nerven, und verzweigen überwiegend im Bereich der dorsalen Neuropile. Die beiden größten Ausläufer mit den weitesten Verzweigungsbereichen ziehen unter anderem in Richtung des Haupt-Beinnervs (Nerv 5) sowie zu den abgehenden Nerven 2-4. Sie verzweigen dorsal und weiter lateral auch bis in den intermediären Bereich. Nahezu alle Fasern tragen deutlich erkennbar Varikositäten.

Physiologie:

Das Neuron CIN-I(d)c1 ist tonisch aktiv bei einer Spontanfrequenz zwischen 30$60 \mathrm{~Hz}$. Die Aktivität ist gekennzeichnet durch rhythmische kurze Salven von meist nur jeweils 2-3 Spikes, gefolgt von je einer inaktiven Phase von ca. 40ms. Das Neuron 
zeigt erhöhte Aktivität sowohl bei taktiler dorsaler Reizung der Tarsen beider Mittelbeine (Abb. 16, A-B) als auch bei Reizung taktiler Haare lateral an beiden Seiten des Abdomens. Die Reaktion auf die Tarsenreizung ist auf beiden Seiten nicht sehr stark, was aber auch damit zusammenhängen kann, dass sich bei diesem Präparat manchmal nur schwache Reflexe auslösen ließen. Es erscheinen kurze Bursts mit nur einigen Spikes bei $100-125 \mathrm{~Hz}$.

Entsprechende Bursts erscheinen jedoch unabhängig von der Art der Reizung nur dann, wenn im Anschluss auch mindestens eines der beiden Mittelbeine - meist das gereizte - bewegt wird. Das Verhalten des Neurons bei kontralateraler Reizung (linkes Mittelbein; Abb. 16, A) ist vergleichbar mit dem bei ipsilateraler Reizung (Abb. 16, B): Es erscheint jeweils ein kurzer Burst, der unmittelbar vor Einsetzen der Muskelaktivität (rechter und/oder linker Depressormuskel) beginnt und während dieser noch eine Zeit lang synchron andauert, in der Regel aber vorher beendet ist. Je länger diese Bursts andauern und je höher die erreichten Frequenzen liegen, desto stärker sind auch die folgenden Muskelaktivitäten. Das zeigt sich besonders deutlich in dem Ausschnitt mit der aktiven Bewegung (Abb. 17, C), wo beide Depressormuskeln zweimal synchrone und stärkere Aktivität zeigen und wo entsprechend längere Bursts des Neurons bei höheren Frequenzen (bis knapp $150 \mathrm{~Hz}$, in der gemittelten Instantanfrequenz entsprechend etwas reduziert) erscheinen. Einer gelegentlich schwächeren Aktivität nur des ipsilateralen Depressormuskels geht hingegen keine merklich erhöhte neurale Aktivität voraus. Hyperpolarisation und Depolarisation des Neurons beeinflusst seine Aktivität in der zu erwartenden Weise, im Experiment gibt es aber keinen Hinweis darauf, dass sich Depolarisation eindeutig auf eine der gemessenen Muskelaktivitäten auswirkt. 


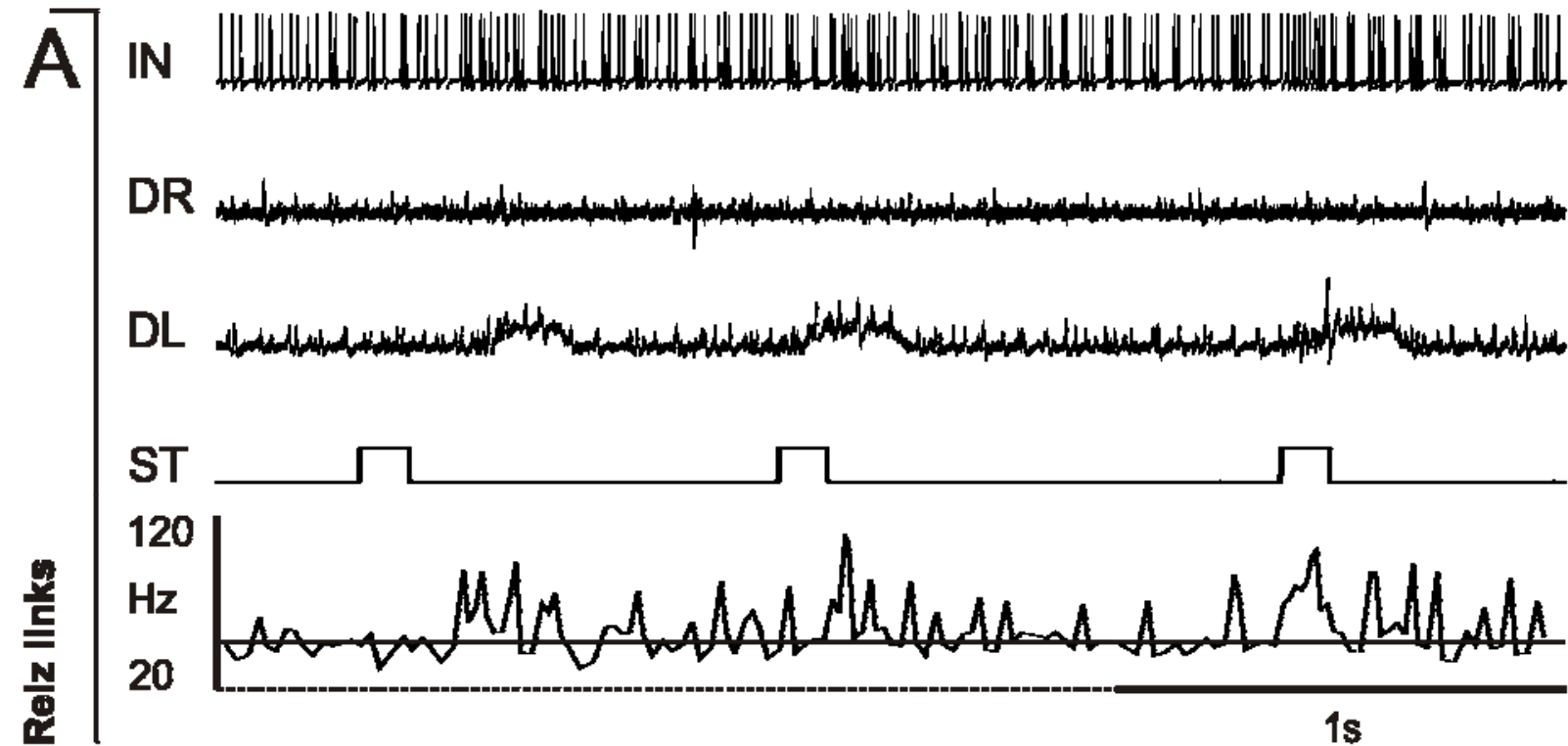

DR

DL

ST

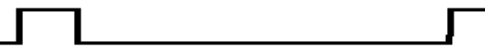
$\Gamma$ 140
$\mathrm{~Hz}$
20

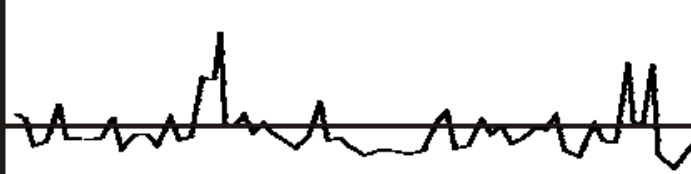
AtAmAAANAAR 1s

Abb. 16: Aktivität, Instantan- und Spontanfrequenzen des Neurons CIN-I(d)c1 und Muskelaktivitäten bei kontralateraler (links) und ipsilateraler Reizung (Mittelbein rechts). Das Neuron (IN) ist rhythmisch-tonisch aktiv, mit kurzen Salven von jeweils 2-3 Spikes, jeweils durch eine etwa 40ms dauernde Pause voneinander getrennt. Dadurch liegt die berechnete Spontanfrequenz hier bei nur knapp $50 \mathrm{~Hz}$. A: Kontralaterale Reizung, dreifacher dorsaler-taktiler Tarsusreiz am linken Mittelbein. Erste Reizung kontinuierlich bis zum Reizerfolg (schwache Reflexe, Zeitraum der Reizung durch gestrichelte Linie in der ST-Spur gekennzeichnet). Nach Reizung erscheint jeweils eine kurze Phase (Burst) schwacher neuronaler Erregung (bei100-125Hz), die kurz vor der Muskelaktivität (Depressor links, DL) beginnt, während dieser synchron noch eine Weile andauert, aber vorher beendet ist. Je länger und hochfrequenter ein Burst, desto stärker die folgende Muskelaktivität. B: Ipsilaterale Reizung, dreifacher dorsal-taktiler Tarsusreiz: Das Verhalten des Neuron während der Reizung ist vergleichbar zur Situation in A, vorzeitig einsetzende und synchron eine Weile andauernde Phasen der Erregung $(100-125 \mathrm{~Hz})$, wobei Dauer und Frequenzen mit der Stärke der Muskelaktivität (Depressor rechts, DR) korreliert sind.

Abkürzungen: IN: Aktivität des Neurons; DR: Depressor trochanteris, rechtes Mittelbein; DL: Depressor tr., linkes Mittelbein; ST: dorsal-taktiler Tarsusreiz. 


\section{CIN-I(d)c1}

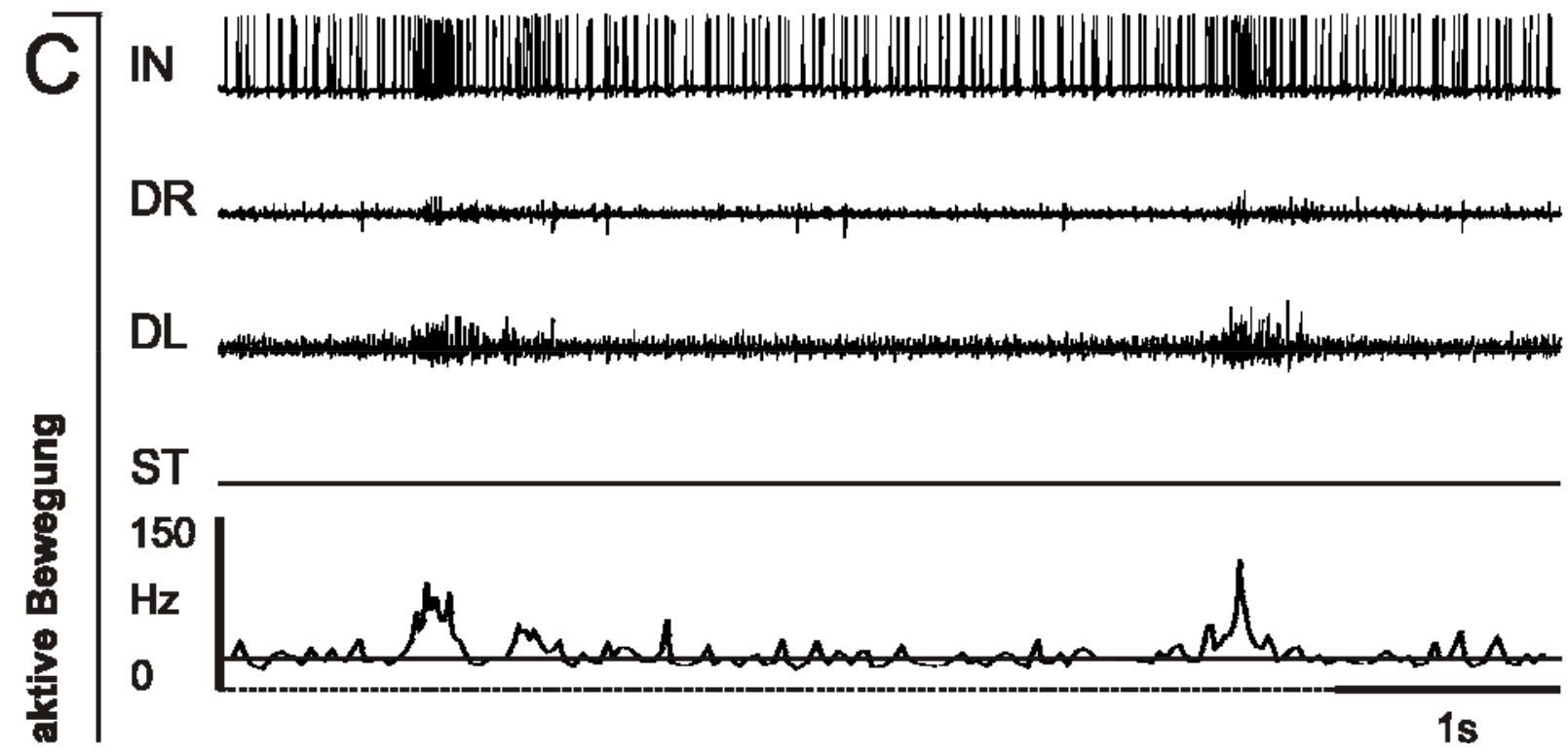

Abb. 17: C: Aktivität, Instantan- und Spontanfrequenzen des Neurons CIN-I(d)c1 und Muskelaktivitäten während spontaner aktiver Bewegung (synchrone Einzelzuckungen beider Depressormuskeln): Auch ohne Reizung erscheinen während spontaner Bewegungen nach vergleichbarem Prinzip wieder die mit der Muskelaktivität korrelierten neuronalen Bursts (IN). In diesem Falle sind beide Depressormuskeln zweimal mehr oder weniger gleichzeitig aktiv. Die Bursts des Neurons beginnen hier jeweils kurz vor dieser stärkeren synchronen Muskelaktivität, sind dann eine Weile synchron aktiv und werden (relativ zum Depressor links, DL) vorzeitig beendet. Die stärkere Muskelaktivität (Depressor links) korreliert mit längerer Dauer und relativ zur momentanen Spontanfequenz von knapp $30 \mathrm{~Hz}$ höherer Instantanfrequenz innerhalb der Bursts (bis knapp $150 \mathrm{~Hz}$, in der Darstellung durch Mittelung entsprechend etwas reduziert).

Abkürzungen: IN: Aktivität des Neurons; DR: Depressor trochanteris, rechtes Mittelbein; DL: Depressor tr., linkes Mittelbein; ST: Reizindikation: keine Reizung.

\section{Kurzdiskussion:}

Aufgrund der morphologischen Eigenschaften des Neurons CIN-I(d)c1 spricht nichts gegen eine möglicherweise bilateral koordinierende Funktion. Das kommissurale Interneuron hat bilaterale Verzweigungsbereiche, die beide relativ weit lateral lokalisiert sind. Dendritische Fasern im ipsilateralen Hemiganglion und varikose Fasern (potentieller lokaler Projektionsbereich) im kontralateralen liegen dabei streng voneinander getrennt vor. Allerdings verzweigen die dendritischen Fasern auf ipsilateraler Seite hauptsächlich dorsal im Bereich beider lateralen Neuropile (pLAC, $\mathrm{aLAC}$ ), eine Region, in welcher typischerweise sensorischer Input von Mechanorezeptoren wie Gelenkstellungsrezeptoren, Chordotonalorganen, campaniformen Sensillen und anderen zu erwarten ist. Die varikosen Fasern auf 
kontralateraler Seite verzweigen ebenfalls dorsal und weit genug lateral, so dass sie an die dorsalen lateralen Neuropile (pLAC, aLAC) heranreichen. Es könnte sich also hier um einen lokalen Projektionsbereich handeln, wo Fasern des Neurons direkt auf Moto- oder Interneurone verschalten. Für eine direkt bilateral koordinierende Funktion des Neurons müssten auf ipsilateraler Seite sensorische Neurone direkt auf lokale Verzweigungen des Neurons verschalten, was zumindest bei den genannten Mechanorezeptoren (Gelenkstellungsrezeptoren, campaniforme Sensillen, Chordotonalorgane und andere) der Falle sein könnte. Es besteht aber auch die Möglichkeit, dass ipsilaterale sensorische Information von taktilen Haaren, die in die ventralen Neuropile projizieren, über lokale Interneurone übermittelt wird. Solche lokalen Interneurone (spikende, SLIN oder nicht-spikende, NSLIN) spielen im Kontext von lokalen Reflexen eine wichtige Rolle, empfangen sensorische Information in den ventralen Neuropilen und projizieren auch in die dorsal-lateralen Neuropile, wo sie direkt auf Motoneurone verschalten können. Möglicherweise liegt hier eine Schnittstelle, wo lokale Reflexe mit bilateralen und/oder intersegmentalen Bewegungsabläufen gekoppelt werden können. Die relevante Information könnte hier somit über lokale Interneurone auf intersegmentale Interneurone übertragen werden. Wenn diese Situation auf das Neuron CIN-I(d)c1 zutrifft, scheint auch eine (indirekt) bilateral koordinierende Funktion möglich. Hyper- oder Depolarisieren des Membranpotentials hatte zwar erwartungsgemäße Auswirkungen auf die neuronale Aktivität, jedoch keinen erkennbaren Einfluss auf die synchron gemessenen Muskelaktivitäten, was zumindest nicht für eine direkt erregende Verschaltung des Neurons auf Motoneurone in einem der beiden Hemiganglien, sondern eher für eine vermittelte Hemmung spricht.

Aufgrund der physiologischen Eigenschaften scheint das Neuron zumindest eine allgemein die Extremitäten koordinierende Funktion zu haben. Es zeigte erhöhte Aktivität während bilateralen und intersegmentalen Reflexen, die durch dorsale taktile Tarsusreize an beiden Mittelbeinen oder durch taktile Reizung von lateral am Abdomen befindlichen Haaren ausgelöst wurden. Diese erhöhte neurale Aktivität erschien im Versuch aber immer nur im Kontext von unmittelbar anschließender Muskelaktivität von mindestens einer der beiden abgeleiteten Depressormuskeln der Mittelbeine, auch, wenn es sich um spontane Bewegungen handelte (Abb. 17, C). Die Neuronbursts erschienen immer wenige Millisekunden vor der einsetzenden Muskelaktivität (ipsi- und kontralateral) und dauerten dann noch eine Weile synchron 
an, waren aber meist vor deren Abklingen beendet. Von der zeitlichen Relation her gesehen und wegen der geschilderten morphologischen Beschaffenheiten des Neurons (Varikositäten und dorsal-lateraler Verzweigungsbereich in der Nähe der kontralateralen dorsalen Neuropile) wäre also eine direkte Verschaltung auf Motoneurone des kontralateralen Depressor trochanteris denkbar. Andererseits wäre bei einem entgegen gerichteten Reflex, bei welchem das ipsilaterale Mittelbein gegen den Untergrund gestemmt und das kontralaterale angehoben wird, eine Hemmung des Neurons während dieser Bewegung zu erwarten, weil ja jeglicher erregender Einfluss auf den kontralateralen Depressormuskel bei dieser Bewegung störend wäre. Eine deutlich gehemmte neurale Aktivität war im Laufe der Aufzeichnungen nicht zu beobachten. Das muss aber nicht bedeuten, dass sie bei bilateralen Reflexen nicht doch auftreten könnte. Das Präparat war zum Zeitpunkt der Aufzeichnungen nicht mehr sehr vital und die ausgelösten Reflexe waren nicht immer deutlich sichtbar. Auch führte die im Falle einer ipsilateralen taktilen Tarsusreizung ausgelöste Anhebbewegung des betreffenden Beins nicht immer zuverlässig zu einem bilateralen Reflex, bei welchem das kontralaterale Bein stärker gegen den Untergrund gestemmt wurde (kontralateraler Depressormuskel aktiv). Nicht selten waren im Anschluss an eine solche Reizung beide Depressormuskeln gleichzeitig aktiv oder beide Mittelbeine wurden synchron angehoben.

Neben dem vermutlichen Projektionsbereich im kontralateralen mesothorakalen Hemiganglion besitzt das Neuron im Metathorakalganglion (ebenfalls kontralaterales Hemiganglion) noch einen weiteren, bei welchem es sich wahrscheinlich auch um einen Projektionsbereich handelt (absteigendes Axon, ebenfalls varikose Fasern). Aus morphologischer Sicht ist es denkbar, dass das Neuron auch hier direkt auf Motoneurone verschaltet. Da aber beim Präparat die Hinterbeine aus den genannten Gründen entfernt werden, können entsprechende intersegmentale Reflexe nicht weiter untersucht und daher auch keine weiteren Aussagen über eine potentiell intersegmentale Koordination in diesem Zusammenhang gemacht werden. Zusammengefasst lässt sich sagen, dass dem Neuron CIN-I(d)c1 wahrscheinlich eine generelle die Extremitäten (vor allem intersegmental) koordinierende Funktion zukommt und dass in diesem Kontext möglicherweise auch eine indirekt bilaterale Koordination der beiden Mittelbeine mit involviert ist. 


\subsubsection{Bilateral verzweigtes kommissurales Typ CIN-I Interneuron CIN-I(d)c2}

Morphologie:

\section{Mesothorakalganglion:}

Das intersegmentale Interneuron $\mathrm{CIN}-\mathrm{I}(\mathrm{d}) \mathrm{c} 2$ besitzt ein bilaterales Verzweigungsmuster und steigt im kontralateralen Konnektiv ab (Abb. 18-19). Der kontralaterale Teil des primären Neuriten und das absteigende Axon sind sehr weit dorsal lokalisiert. Die Verzweigungsbereiche sind in beiden Hemiganglien auf intermediärem Niveau anzutreffen und erstrecken sich auf beiden Seiten jeweils von der inneren Randzone der ipsilateralen sich gegenüberliegenden Konnektive (gedachte Verbindungslinie durch das Ganglion auf Höhe des inneren Randes der Konnektive) bis relativ weit nach lateral, aber nicht extrem weit. Die Verzweigungen auf kontralateraler Seite sind überwiegend varikos, während die ipsilateralen Fasern dendritischen Charakter haben. Das Neuron besitzt morphologisch betrachtet eine große Ähnlichkeit zu dem Interneuron 325, das von RAMIREZ und PEARSON (1988) beschrieben wurde.

Das kleinere Soma $(\varnothing=15 \mu \mathrm{m})$ liegt median-ventral, auf Höhe nahe der inneren Randzone der ipsilateralen (linken) Konnektive (gedachte Verbindungslinie). Der primäre Neurit verläuft in einem Bogen zunächst etwas nach lateral und dann zunehmend nach anterior. Dabei steigt er in den intermediären Bereich auf. Etwa auf Höhe relativ zur Querachse durch den Mittelpunkt des Ganglions beginnt der deutlich verdickte kommissurale Prozess, von welchem ipsilateral alle dendritischen Hauptund Nebenverzweigungen ausgehen.

Die erste Hauptverzweigung zieht nach lateral, teilt sich jedoch schon wenige Mikrometer entfernt in einen nach lateral und einen nach posterior-lateral verlaufenden Verzweigungsbereich. Beide Äste sind intermediär gelegen, in etwa in der Region, in welcher die dorsalen Trakte (DIT, DMT) verlaufen.

Die zweite Hauptverzweigung zieht ebenfalls auf intermediärem Niveau nach anterior-lateral, reicht jedoch genau wie die erste Hauptverzweigung nicht extrem weit nach lateral. Im anschließenden Stück trägt der Neurit auf ipsilateraler Seite noch drei kleinere Nebenverzweigungen. Zwei davon verlaufen nach anterior, die etwas größere Dritte zieht nach posterior über das ventral gelegene Soma hinweg. Alle drei Nebenverzweigungen liegen im intermediären bis dorsalen Bereich, keine 

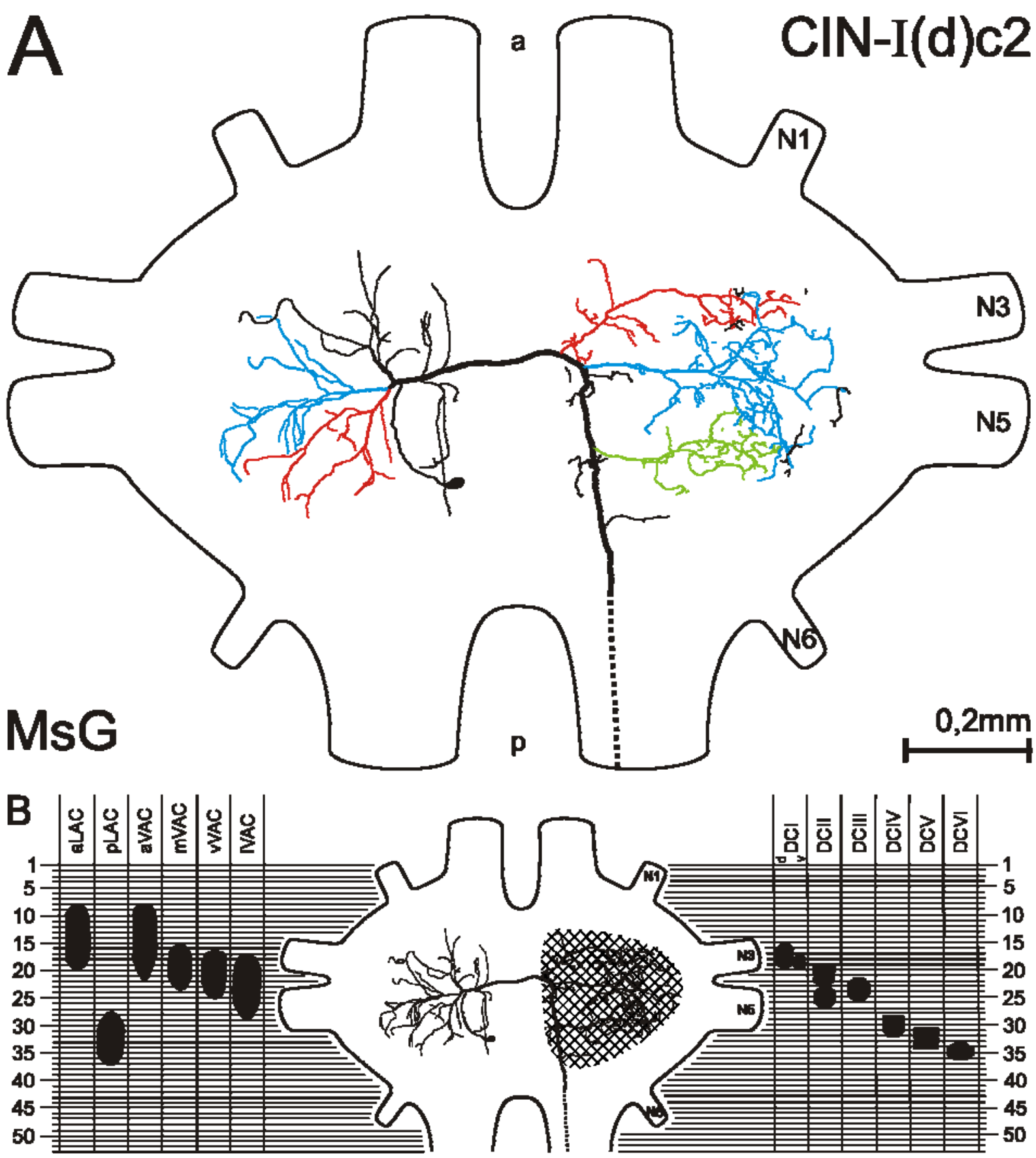

Abb. 18: Intersegmentales Interneuron CIN-I(d)c2 mit bilateralem Verzweigungsmuster (Typ I). A: Gezeichnete 2D-Projektion, Mesothorakalganglion. Dorsal im kontralateralen (rechten) Konnektiv absteigendes Axon. Das kleinere Soma $(\varnothing=15 \mu \mathrm{m})$ liegt median-ventral, nahe der gedachten Verbindungslinie auf Höhe der inneren Randzone der (linken) Konnektive. Die Verzweigungen des ipsilateralen Hemiganglions sind von dendritischem Charakter, erstrecken sich von ventral bis intermediär und liegen im Bereich der ventralen Neuropile (mVAC und möglicherweise auch IVAC). Der primäre Neurit durchquert wahrscheinlich die dorsale Kommissur DCll und steigt im kontralateralen Hemiganglion extrem weit dorsal auf. Auf dorsal-medianer Höhe verläuft er dort bogenförmig in posteriore Richtung. Das Axon trägt mehrere, sehr große, dorsal gelegene Kollaterale, die weit nach lateral in den Bereich der lateralen Neuropile (aLAC, pLAC) ziehen. Ihre Verzweigungen sind überwiegend varikos, es handelt sich hier also vermutlich um lokale Projektionsbereiche. B: Übersicht desselben Neurons und Vergleich mit der relativen Lage wichtiger morphologischer Strukturen (aus: PFLÜGER et al. 1988; leicht modifiziert).

Abkürzungen: MsG: Mesothorakalganglion; a: anterior; p: posterior; N1-N6: Nerv1-6; Kreuzschraffur in $\mathbf{B}$ : Bereich, in welchem überwiegend varikose Fasern (potentiell lokale Projektionsbereiche) vorkommen; Abkürzungen in B Siehe Tabelle der verwendeten Abkürzungen. 

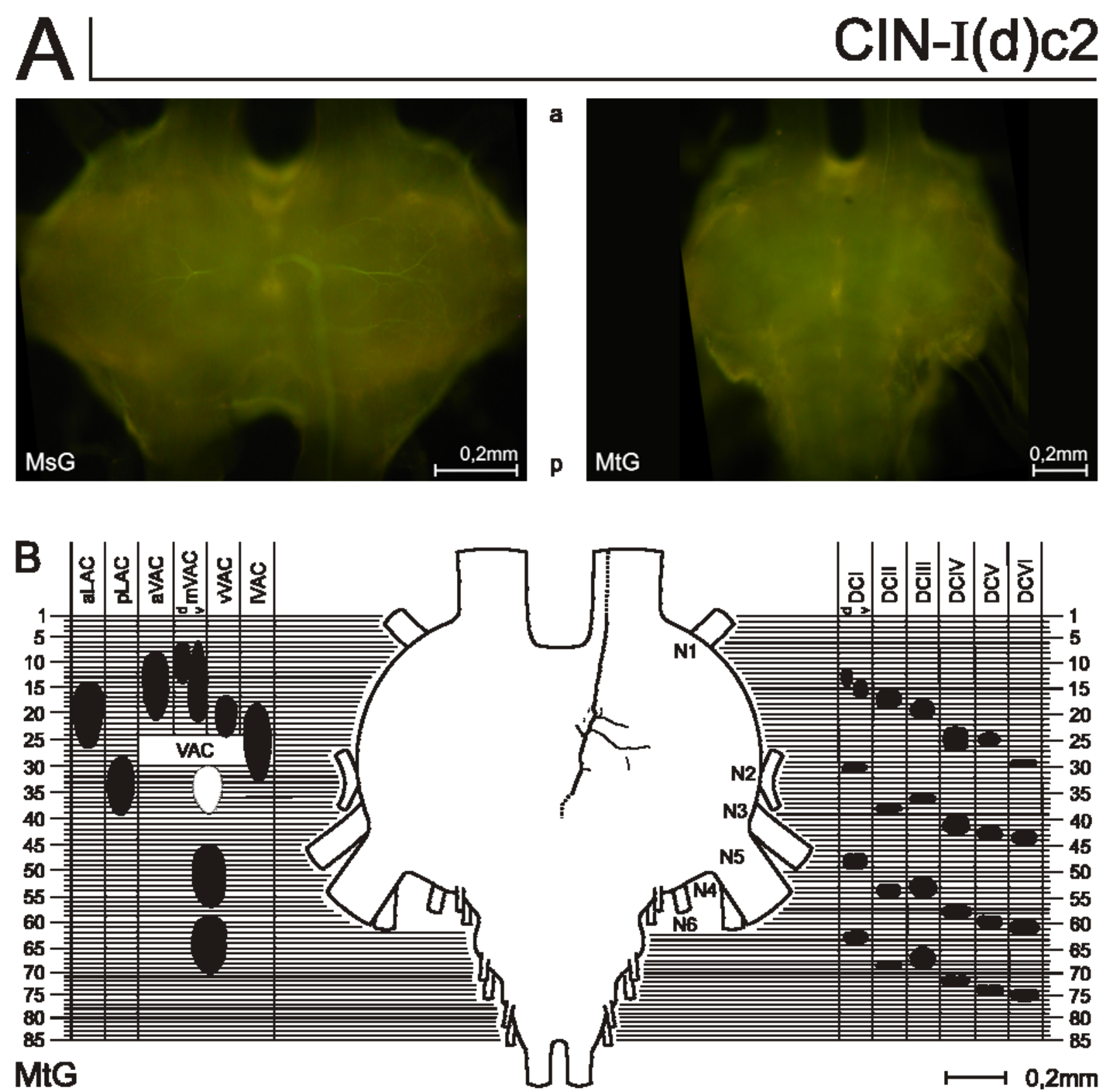

Abb. 19: A: Originalfotografien des Neurons CIN-I(d)c2. MsG: Mesothorakalganglion, dorsale Ansicht. Die bilateral angeordneten Hauptverzweigungsbereiche liegen in unterschiedlichen Tiefenregionen. Die eher dendritischen Verzweigungen des ipsilateralen (linken) Hemiganglions sind im intermediären bis ventralen Bereich, in der Umgebung der ventralen Neuropile (mVAC, IVAC) lokalisiert, die varikosen Fasern auf kontralateraler Seite verzweigen dorsal in der Nähe der lateralen Neuropile (aLAC, pLAC). Das Axon steigt dorsal im kontralateralen (rechten) Konnektiv ab. Der primäre Neurit durchquert die weiter dorsal gelegene Kommissur DCII und ist daher nicht im Fokus. Das kleinere Soma $(\varnothing=15 \mu \mathrm{m})$ liegt median-ventral im linken Hemiganglion. Es fluoresziert nur schwach und ist deshalb in dieser Fokal-Ebene nicht erkennbar. MtG: Metathorakalganglion, dorsale Ansicht. Das einlaufende Axon ist anterior im kontralateralen (rechten) Hemiganglion und im Bereich des Konnektivs zu erkennen. Die weitere Verzweigung ist im Metathorakalganglion aufgrund schwacher Fluoreszenz in Einzelbildern nicht zu verfolgen. B: Erst in einer gezeichneten 2DProjektion werden einzelne der Hauptstrukturen sichtbar (aus: PFLÜGER et al. 1988; leicht modifiziert). Zwei größere Ausläufer ziehen von dorsal-median nach lateral, aber es bleibt unklar, wie sie weiterverzweigen, ob sie bis an die lateralen Neuropile heranreichen und ob sie varikos sind. Auch der terminale Teil des Axons ist nicht bis zum Ende zu verfolgen. Der Neurit könnte über eine Kommissur oder einen kommissuralen Trakt über die Mittellinie hinweg und von dort bis in den Bereich der angelagerten Abdominalganglien oder noch weiter ziehen.

Abkürzungen: MsG: Mesothorakalganglion; MtG: Metathorakalganglion; a: anterior; p: posterior; N1N6: Nerv1-6; Morphologische Abkürzungen in B Siehe Tabelle der verwendeten Abkürzungen. 
verläuft jedoch über die Mittellinie hinweg. Der primäre Neurit steigt dann leicht etwas in dorsaler Richtung an und zieht zu den dorsalen Kommissuren DCII-DCIII, von welchen er wahrscheinlich die weiter dorsal gelegene Kommissur DCII durchquert.

Auf kontralateraler Seite verläuft der hier immer noch verdickte Teil des Neuriten bogenförmig nach posterior (etwa auf Höhe der inneren Randzone der kontralateralen Konnektive), steigt dabei sehr weit dorsal auf und geht in das im kontralateralen (rechten) Konnektiv absteigende Axon über, das ebenfalls äußerst dorsal verläuft. Das Axon trägt über einen weiten Bereich, der schon am bogenförmigen Verlauf des primären Neuriten beginnt, einige kleine und drei sehr große Kollaterale. Die kleineren verzweigen sowohl zu lateraler wie medianer Seite, es gibt jedoch keine kommissuralen Anteile. Die erste große Kollaterale zieht auf dorsalem Niveau zunächst schräg nach anterior und verzweigt dann lateral, wobei sie sich zunehmend wieder bis in den intermediären Bereich erstreckt. Dieses Prinzip gilt auch für die anderen beiden großen Kollaterale, die ebenfalls lateral verzweigen. Die Verzweigungen aller drei großen Kollaterale überlappen zum Teil und dürften bis in den Bereich der dorsalen lateralen Neuropile (pLAC, aLAC) hineinreichen.

\section{Metathorakalganglion:}

Das Axon zieht relativ weit dorsal im kontralateralen (rechten) Konnektiv in das Metathorakalganglion hinein, verläuft dann nach posterior und nähert sich dabei immer noch auf dorsalem Niveau - zunehmend der Mittellinie an (Abb. 19). Die Färbung ist in diesem Ganglion nur schwach ausgeprägt, so dass nichts darüber ausgesagt werden kann, ob und wenn welche Kommissur oder welchen kommissuralen Trakt das Neuron durchquert und wie es weiterverzweigt. Auf kontralateraler Seite (rechtes Hemiganglion) scheint sich auf dorsalem Niveau ein größerer Verzweigungsbereich nach lateral in Richtung des abgehenden Nerven N2 fortzusetzen, aber die terminalen Strukturen sind hier ebenfalls nicht deutlich zu erkennen und es bleibt unklar, ob Varikositäten auftreten. 
Physiologie:

Das Neuron $\mathrm{CIN}-\mathrm{I}(\mathrm{d}) \mathrm{c} 2$ ist rhythmisch-tonisch aktiv. Die rhythmischen Salven umfassen zwei bis wenige Spikes bei Frequenzen zwischen meist $100-125 \mathrm{~Hz}$. Nach jeder Salve folgt eine kurze Phase („Pause“) von meist 25-50ms, in welcher keine Spikes erscheinen. Die jeweils berechneten Spontanfrequenzen liegen daher niedriger bei meist $30-50 \mathrm{~Hz}$. Sowohl dorsale taktile Tarsusreize (Abb. 20, A; Abb. 21, C) an beiden Mittelbeinen als auch passives Anheben (Abb. 20, B) beider Mittelbeine (wenn die ausgelösten Reflexe nur schwach sichtbar waren) haben einen Einfluss auf die Neuronaktivität. Dabei ist das neuronale Verhalten bei kontralateraler und ipsilateraler Reizung vergleichbar, im Falle der ipsilateralen Reizung (linkes Mittelbein) jedoch ausgeprägter (Abb. 20, A). In jedem Fall erscheinen in zeitlicher Korrelation zur Muskelaktivität neuronale Bursts, die sich durch Spikes kleinerer Amplitude auszeichnen (Abb. 20, A; Abb. 21, D; schwarze Pfeile). Diese Spikes sitzen ihrerseits einem dauerhaft depolarisierten DC-Potential auf (möglicherweise ein EPSP). Solche Bursts erscheinen nach taktiler Tarsusreizung sowohl des kontralateralen (Abb. 21, C) als auch des ipsilateralen Mittelbeins (Abb. 20, A), während ipsilateral passiven Anhebens (Abb. 20, B) und auch während aktiven Bewegungen ohne vorherige Reizung (Abb. 21, D). Dabei steigt die Instantanfrequenz auf $100 \mathrm{~Hz}$ bis maximal knapp $200 \mathrm{~Hz}$ an. Immer wenn im Anschluss an eine Reizung Aktivität in einem oder beiden der abgeleiteten Muskeln auftritt, sind diese neuronalen Bursts streng alternierend dazu korreliert und erscheinen unmittelbar nach beendeter Aktivität entweder vom kontra- oder vom ipsilateralen Depressor (besonders deutlich in Abb. 21, C-D). Während einer spontanen Bewegungssequenz (Abb. 21, D) erscheint wiederholt stärkere Aktivität des ipsilateralen Depressors und mehr oder weniger synchron dazu latent schwache Aktivität vom kontralateralen Depressormuskel. Hierbei treten die neuronalen Bursts streng alternierend zur ipsilateralen Depressoraktivität auf. Die kleineren Spikes treten nur innerhalb dieser Bursts auf und haben eine Amplitude, die fast nur halb so groß ist wie die für dieses Neuron kennzeichnende Spike-Amplitude. Sie haben untereinander aber eine nahezu konstante Amplitude, es erfolgt also keine allmähliche Verminderung. Dies ist eine Situation, wie sie vergleichbar bereits für das Neuron $\mathrm{CIN}-\mathrm{I}(\mathrm{a}) \mathrm{c} 1$ beschrieben wurde. Auch hier erreichen die kleineren Spikes fast nur die Hälfte der Ausgangsamplitude und treten nur innerhalb eines Burst auf, der 


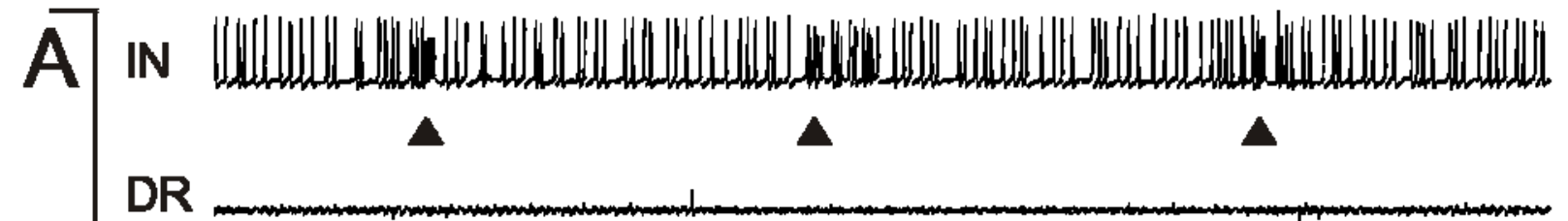

DL

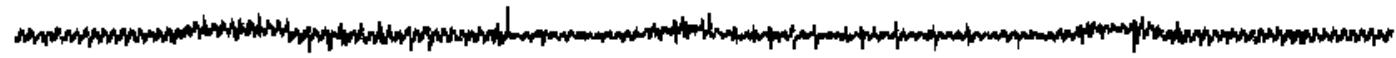
ST $几 几$ 180

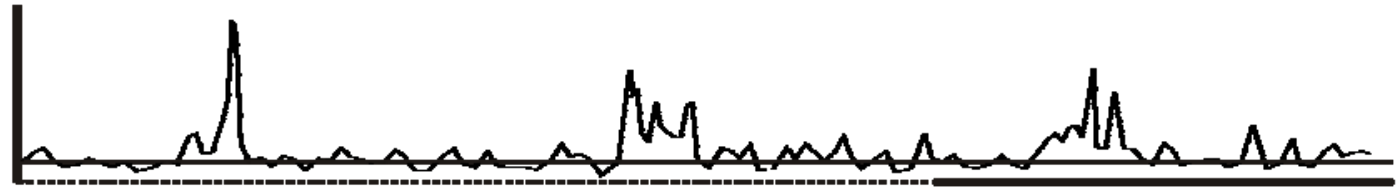
20 1s

DR

L

P $\sqrt{2}$ z

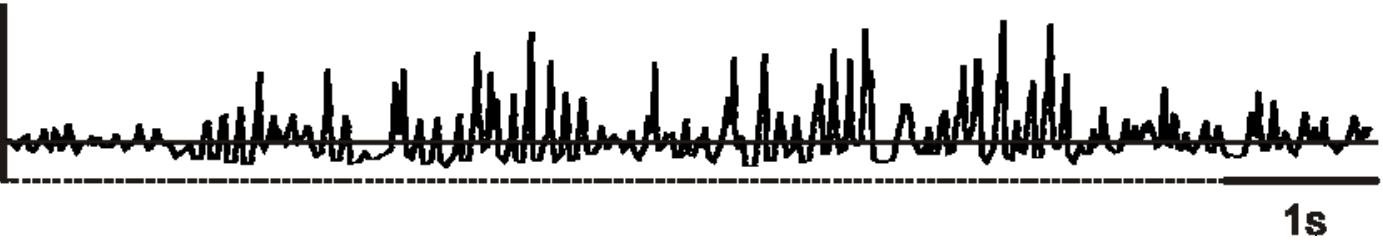

Abb. 20: Aktivität, Instantan- und Spontanfrequenzen des Neurons CIN-I(d)c2 und Muskelaktivitäten bei ipsilateraler Reizung (Mittelbein links). Das Neuron (IN) ist rhythmisch-tonisch aktiv, mit kurzen Salven von wenigen Spikes, jeweils durch eine etwa 25-50ms dauernde Pause voneinander getrennt. Dadurch liegen die berechneten Spontanfrequenzen zwischen $30-50 \mathrm{~Hz}$. A: Dreifacher dorsal-taktiler Tarsusreiz (linkes Mittelbein): Nach jeder Reizung erscheint jeweils ein kurzer neuronaler Burst $(100-200 \mathrm{~Hz}$ ), in welchem Spikes geringerer Amplitude erscheinen (schwarze Pfeile). Die Korrelation zur Muskelaktivität (DR, DL) wird hier nicht deutlich, da diese hier nur äußerst schwach ist. B: Passives Anheben (Mittelbein links): Neben den genannten Bursts mit Spikes kleinerer Amplitude und vergleichbaren Frequenzen (bis max. $200 \mathrm{~Hz}$ ) treten während der gesamten Auslenkung zum Teil deutlich längere Verzögerungen zwischen den Bursts auf, so dass die rhythmische Aktivität des Neurons ausgeprägter erscheint. Synchron zur neuronalen Aktivität erscheint im Zuge des ausgelösten Widerstandsreflexes eine dauerhafte Aktivität des ipsilateralen Depressors (DL) für die Dauer des Anhebens.

Abkürzungen: IN: Aktivität des Neurons; DR: Depressor trochanteris, rechtes Mittelbein; DL: Depressor tr., linkes Mittelbein; ST: dorsal-taktiler Tarsusreiz; SP: passives Anheben. 


\section{$\mathrm{CIN}-\mathrm{I}(\mathrm{d}) \mathrm{c} 2$}
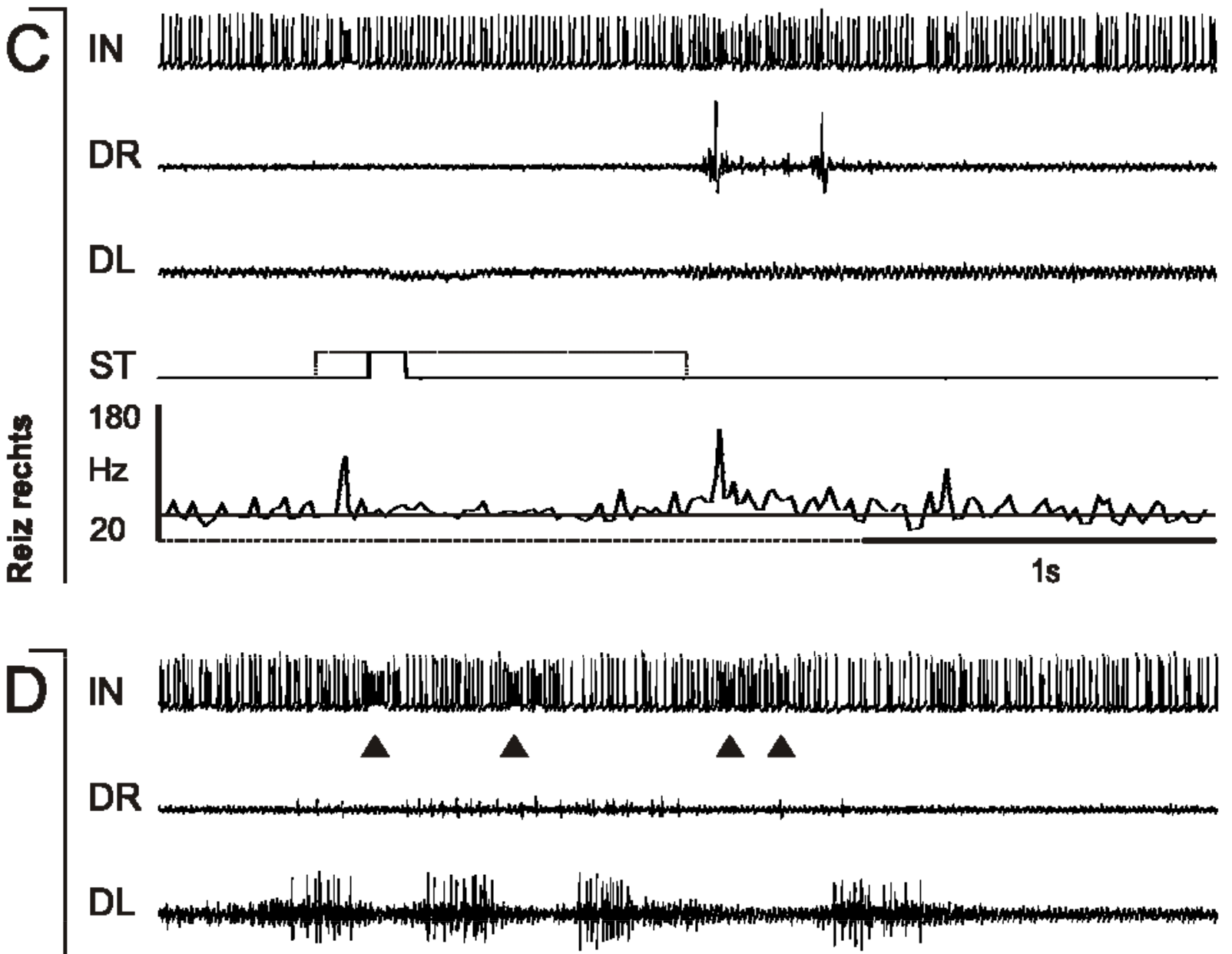

ST

Abb. 21: Aktivität, Instantan- und Spontanfrequenzen des Neurons CIN-I(d)c2 und Muskelaktivitäten bei kontralateraler Reizung (Mittelbein rechts) und spontaner Bewegung (Einzelzuckungen beider Depressormuskeln, aber kein Laufen). C: Einmaliger dorsal-taktiler Tarsusreiz: Reizindikation nicht zeitgenau, kontinuierliche Reizung bis zum Reizerfolg (schwache Reflexe, Zeitraum der Reizung durch gestrichelte Linie in der ST-Spur angegeben). Nach der Reizung erscheint Aktivität des kontralateralen Depressors (DR, Aktivität der slow- und der fast-unit, 2 einzelne Spikes). Unmittelbar nach dem ersten Spike der fast-unit erscheint in alternierender Korrelation zur Muskelaktivität ein neuronaler Burst (IN, max. $160 \mathrm{~Hz}$ ), in welchem Spikes geringerer Amplitude auftreten. Dieses Verhalten ist bei kontralateraler Reizung aber weniger deutlich ausgeprägt als bei ipsilateraler Reizung (Abb. 20). D: Spontane Bewegung beider Mittelbeine: Hier tritt mehrmals stärkere Aktivität des ipsilateralen (DL) und mehr oder weniger synchron schwächere Aktivität des kontralateralen Depressors (DR) auf. Die neuronalen Bursts (Spikes mit kleiner Amplitude, schwarze Pfeile) erscheinen jeweils streng alternierend korreliert unmittelbar im Anschluss an beendete Aktivität des ipsilateralen Depressors und erreichen Frequenzen zwischen $100-200 \mathrm{~Hz}$.

Abkürzungen: IN: Aktivität des Neurons; DR: Depressor trochanteris, rechtes Mittelbein; DL: Depressor tr., linkes Mittelbein; ST: dorsal-taktiler Tarsusreiz; SP: passives Anheben. 
zeitlich jeweils unmittelbar nach beendeter Muskelaktivität beginnt. Der einzige Unterschied ist, dass sich die neuronalen Bursts nicht in zwei verschiedene Phasen einteilen lassen. In jedem Burst erscheinen nur Spikes mit geringerer Amplitude.

Passives Anheben des ipsilateralen (linken) Mittelbeins bewirkt darüber hinaus noch, dass die einzelnen Bursts durch größere inaktive Phasen zwischen ihnen mehr verzögert aufeinander folgen (Abb. 20, B). Dadurch erscheint die neuronale Aktivität noch rhythmischer ausgeprägt als im Falle der taktilen Tarsusreize oder während aktiver Bewegungen der Mittelbeine ohne Reizung.

Hyperpolarisation und Depolarisation beeinflusst die neuronale Aktivität in der zu erwartenden Weise. Depolarisation hatte jedoch keine sichtbare Wirkung auf die gemessene Muskelaktivität.

Kurzdiskussion:

Das Neuron CIN-I(d)c2 ähnelt sowohl morphologisch als auch physiologisch dem bereits beschriebenen Interneuron 325 (RAMIREZ and PEARSON 1988). Letzteres zeigt eine im Kontext von Laufbewegungen streng rhythmische Aktivität und ist während des Fluges inaktiv. Auch das Neuron CIN-I(d)c2 zeigt tonisch-rhythmische Aktivität und ist im Kontext von spontanen Bewegungen und ausgelösten Reflexen an beiden Mittelbeinen aktiv (Abb. 20-21). Die dabei auftretenden neuronalen Bursts sind ebenfalls streng mit der gemessenen Muskelaktivität (Depressormuskeln beider Mittelbeine) korreliert, und zwar alternierend nach kontralateraler taktiler Reizung und während spontaner rhythmisch synchroner Bewegungen beider Mittelbeine. Da es sich um ein größeres intersegmentales Interneuron handelt, ist anzunehmen, dass es auch eine intersegmental koordinierende Funktion hat. Das Neuron steigt im kontralateralen Konnektiv $a b$ und verzweigt dort im kontralateralen und möglicherweise auch im ipsilateralen metathorakalen Hemiganglion (Abb. 18-19). Dabei liegen die Verzweigungen auf kontralateraler Seite dorsal und ziehen lateral in Richtung des anterior gelegenen lateralen Neuropils ( $a L A C)$. Es handelt sich also hier vermutlich um einen Projektionsbereich des Neurons. Über eine mögliche intersegmentale Koordination kann allerdings wegen der bei der Präparation entfernten Hinterbeine und auch aufgrund unvollständiger Färbung im Metathorakalganglion nichts Genaueres ausgesagt werden. Lediglich dorsale taktile Reizung der beiden Mittelbeintarsen und passives Anheben der Mittelbeine hatte 
einen deutlichen Einfluss auf die neuronale Aktivität. Das kann aber auch damit zusammenhängen, dass die am Präparat ausgelösten Reflexe manchmal nur schwach erkennbar waren. Aufgrund der Morphologie der im Mesothorakalganglion bilateralen Verzweigung ist eine direkt bilateral koordinierende Funktion des Neurons denkbar. Die überwiegend dendritischen Verzweigungen der ipsilateralen Seite sind in intermediärem bis ventralen Bereich in der Nähe der ventralen Neuropile (mVAC, IVAC) in einer Region gelegen, in welcher sensorischer Input von taktilen Haaren zu erwarten ist. $\mathrm{Da}$ die überwiegend varikosen Fasern der kontralateralen Verzweigungen bis in die Region der dorsalen lateralen Neuropile (aLAC, pLAC) hineinreichen, ist hier eine direkte synaptische Verschaltung des Interneurons auf Motoneurone theoretisch möglich (Abb. 18, A-B). Es ist aber unwahrscheinlich, dass das Neuron kontralateral exzitatorisch auf Motoneurone des Depressormuskels verschaltet, da die neuronalen Bursts ja immer erst nach der beendeten Muskelaktivität erscheinen (alternierende Korrelation). Auch erscheint neuronale Erregung nicht ausschließlich während unidirektional in einer Richtung ablaufender Reflexe, sondern in beiden Richtungen. Bei einer direkt und ausschließlich bilateral koordinierenden Funktion mit direkter Verschaltung auf Motoneurone in nur einem der Hemiganglien wäre eine Hemmung des Neurons während Reflexen zu erwarten, die in der entgegen gesetzten Richtung ablaufen. Wenn das Neuron beispielsweise kontralateral direkt den Depressor trochanteris ansteuert, dann wäre seine Aktivierung störend, wenn durch einen dorsalen taktilen Tarsusreiz ein Reflex ausgelöst wird, bei dem das betreffende Mittelbein gerade angehoben wird (Trochanter-Levator aktiv). Solch eine unidirektional auftretende Hemmung des Neurons ist nicht $\mathrm{zu}$ beobachten. Auch spricht das rhythmisch-tonische Aktivitätsmuster des Neurons, das während passivem Anheben des ipsilateralen Mittelbeins noch deutlicher akzentuiert ist (Abb. 18, B: Latenzen zwischen den SpikeSalven werden größer), gegen eine rein bilateral koordinierende Funktion. Wahrscheinlicher ist eine im Kontext der intersegmentalen Koordination integrierte bilaterale Koordination. Diese Situation trifft auch noch auf andere der gefundenen kommissuralen Interneurone mit bilateralem Verzweigungsmuster vom Typ CIN-I zu und es ist denkbar, dass dies auch ein generelles bei vielen großen und intersegmentalen (kommissuralen) Interneuronen (bei unterschiedlichen Tiergruppen) vorkommendes Merkmal ist. Beispielsweise trifft dies auf eine für Wirbeltiere beschriebene Klasse kommissuraler Interneurone zu, welchen ebenfalls 
eine Funktion sowohl im Kontext der bilateralen wie intersegmentalen Koordination zugeschrieben wird (ICINs: „long range“ CINs: BUTT et al. 2002; BANNATYNE et al. 2003; BUTT and KIEHN 2003; JANKOWSKA et al. 2003, 2005a; KRUTKI et al. 2003; EDGLEY et al. 2004; HAMMAR et al. 2004). Eine weitere gelegentlich wie bei diesem Neurontyp vorkommende Eigenschaft ist das Auftreten von Spikes unterschiedlicher Amplitude in ein und derselben Neuron-Ableitung, vergleichbar zum Neuron CIN-I(a)c1(Abb. 20-21). Es ist nicht auszuschließen, dass trotz einer intrazellulären Ableitung (erfolgreiche Färbung, Hyper- und Depolarisieren des Membranpotentials hatte die zu erwartenden Effekte) die Aktivität eines anderen Neurons mitregistriert wurde. Aber die Spikes mit der kleineren Amplitude erschienen ausschließlich in den Neuronbursts, zeitlich streng korreliert zu den genannten spontanen Muskelzuckungen und auch klar in einer separaten „Phase“ von den Spikes mit Ausgangs-Amplitude getrennt. Eine Modulation der Amplitude über präsynaptische Hemmung durch ein anderes Interneuron ist unwahrscheinlich, da keine allmählich zunehmende Abschwächung der Amplitude zu beobachten ist. Die Spikes mit kleinerer Amplitude sind klar von solchen mit Ausgangsamplitude abgegrenzt und untereinander nahezu konstant. Eine weitere mögliche Ursache für den beobachteten Effekt könnte das Vorhandensein mehrerer Triggerzonen sein, wie dies von großen intersegmentalen Interneuronen bekannt ist (HUSTERT 1985). Zwar sollten die biophysikalischen Vorgänge bei der Fortleitung eines Aktionspotentials an der Membran (Kaskade der Ladungsverschiebungen) und damit die Spike-Amplitude bei konstanter Ableitstelle unabhängig vom Ort der Entstehung der Spikes konstant bleiben, solange kein modulierender Einfluss über ein anderes Interneuron stattfindet. Andererseits ist aber nicht zu erwarten, dass Aktionspotentiale, die - in der Triggerzone des Neurons in einem anderen Ganglions generiert - über ein Axon mit großem Durchmesser und über weitere Strecken von entfernten Verzweigungsbereichen des Neurons zum jeweiligen Projektionsbereich geleitet werden, auch wieder bis in die feinsten dendritischen Fasern lokal in einem anderen Verzweigungsbereich (in einem anderen Ganglion) aktiv fortgeleitet werden. Möglicherweise handelt es sich bei den beobachteten Spikes mit kleinerer Amplitude um einen sich in Verzweigungen mit dünnerem Durchmesser passiv und elektrotonisch ausbreitenden Effekt, der daher rührt, dass Aktionspotentiale weiter von der Ableitstelle entfernt durch das Axon geleitet werden. Andererseits sitzen die Spikes mit kleinerer Amplitude auch bei diesem Neuron (vergleichbar wie beim 
Neuron CIN-I(a)c1, Abb. 13, B) einem dauerhaft depolarisierendem DC-Potential auf, was eher dafür spricht, dass die Spikes in der näheren Umgebung der Ableitstelle ausgelöst worden sein müssen. Denn bei letzterem kann es sich nur um ein einzelnes oder mehrere aufsummierte postsynaptische Potentiale handeln, deren Amplitude bei der Fortleitung über die Membran schnell abgeschwächt wird (abhängig von der Membran-Längskonstante). Dennoch ist dies ein in großen intersegmentalen und kommissuralen Interneuronen manchmal auftretendes Phänomen, das aufgrund der strengen Korrelation zu Muskelaktivitäten während Bewegungen von Extremitäten möglicherweise eine wichtige Rolle im Kontext von intersegmentaler und/oder bilateraler Koordination spielt. Anhand der vorliegenden morphologischen und physiologischen Daten kann dazu jedoch nichts Genaueres ausgesagt werden.

Zusammengefasst lässt sich sagen, dass das Neuron höchstwahrscheinlich eine allgemein die Extremitäten koordinierende Funktion besitzt. Es zeigt eine Aktivität, die streng zu Muskelaktivitäten während Bewegungen der Extremitäten (Mittelbeine) korreliert ist. Sehr auffällig ist in diesem Zusammenhang das Auftreten von Spikes unterschiedlicher Amplitude in der Neuronableitung. Durch die morphologische Struktur seiner mesothorakal bilateralen Verzweigungsbereiche scheint es auch eine bilateral koordinierende Funktion zu haben, die allerdings vermutlich in den Kontext einer intersegmentalen Koordination eingebunden ist, da das Neuron auch im Metathorakalganglion verzweigt.

\subsubsection{Median verzweigtes kommissurales Typ CIN-II Interneuron CIN-II(ad)c1}

Morphologie:

\section{Mesothorakalganglion:}

Das intersegmentale Interneuron $\mathrm{CIN}-\mathrm{II}(\mathrm{ad}) \mathrm{c} 1$ ist durch einen median gelegenen Hauptverzweigungsbereich (Typ CIN-II) gekennzeichnet und besitzt ein im kontralateralen (rechten) Konnektiv durchlaufendes Axon (Abb. 22-23). Von der aufsteigenden Hauptkollaterale entspringt eine größere Seitenverzweigung, die im kontralateralen Hemiganglion auf dorsaler Höhe bis an das posterior-laterale Neuropil (pLAC) heranreicht. Der primäre Neurit durchquert dorsal eine der 
posterioren dorsalen Kommissuren DCV oder DCVI.

Das Soma hat einen Durchmesser von etwa $20 \mu \mathrm{m}$ und liegt median-ventral im linken Hemiganglion, auf der relativen Höhe des inneren Randbereichs der ipsilateralen Konnektive (gedachte longitudinale Verbindungslinie). Der primäre Neurit zieht zunächst nach lateral in Richtung von Nerv N5 bis auf die Höhe der gedachten Vertikalen, die zentral durch die ipsilateralen Konnektive verläuft. Dabei steigt er in den intermediären Bereich auf und verläuft anschließend bogenförmig nach median und weiterhin ansteigend in Richtung der hinteren dorsalen Kommissuren (DCVDCVI). Eine erste größere Nebenverzweigung zieht vom primären Neuriten nach posterior und verläuft dann oberhalb des Somas, wo sie dorsal weiterverzweigt. Kurz vor dem Durchtritt durch die Kommissur bildet der Neurit einen verdickten Prozess, von dem ausgehend alle weiteren ipsilateralen Hauptverzweigungen ihren Ursprung nehmen. Die erste Hauptverzweigung verläuft nach anterior und verzweigt intermediär bis dorsal-median im näheren Umfeld der vorderen dorsalen Kommissuren DCI-DCIII. Sie trägt keine kommissuralen Ausläufer.

Etwa $15 \mu \mathrm{m}$ weiter, unmittelbar vor der Kommissur, teilt sich der verdickte Prozess des Neuriten in den kommissuralen und einen ipsilateralen, ein Stück weit in die Tiefe ziehenden Teil. Von letzterem ausgehend verteilen sich ipsilateral die weiteren vier Hauptverzweigungen (Hauptverzweigungen zwei bis fünf) mehr oder weniger sternförmig über einen größeren, median gelegenen Bereich.

Die zweite Hauptverzweigung zieht auf intermediärem Niveau ebenfalls nach anterior und teilt sich in einen ipsilateralen Seiten- und einen kontralateralen Hauptzweig. Der Seitenzweig verläuft weit nach ipsilateral und verzweigt intermediär. Der Hauptabzweig verläuft noch ein Stück zur Ganglionmitte hin, passiert eine der dorsalen Kommissuren DCIII oder DCIV und verzweigt kontralateral ebenfalls intermediär bis dorsal-median in der Umgebung der dorsalen Kommissuren. Er trägt einen rekurrenten Ausläufer, der intermediär über die dorsale Kommissur DCIII zurück in das ipsilaterale Hemiganglion zieht und mit lokalen Fasern der ersten Hauptverzweigung überlappt.

Die dritte Hauptverzweigung verläuft nach lateral in die gleiche Richtung wie der kontralaterale Teil des primären Neuriten, ist jedoch etwas tiefer gelegen und verzweigt zum größten Teil intermediär, teilweise auch dorsal weiter. Ihre Verzweigungen erstrecken sich bis etwas über die Stelle hinweg, an welcher sich das Axon in die auf- und die absteigende Hauptkollaterale teilt. Die vierte 

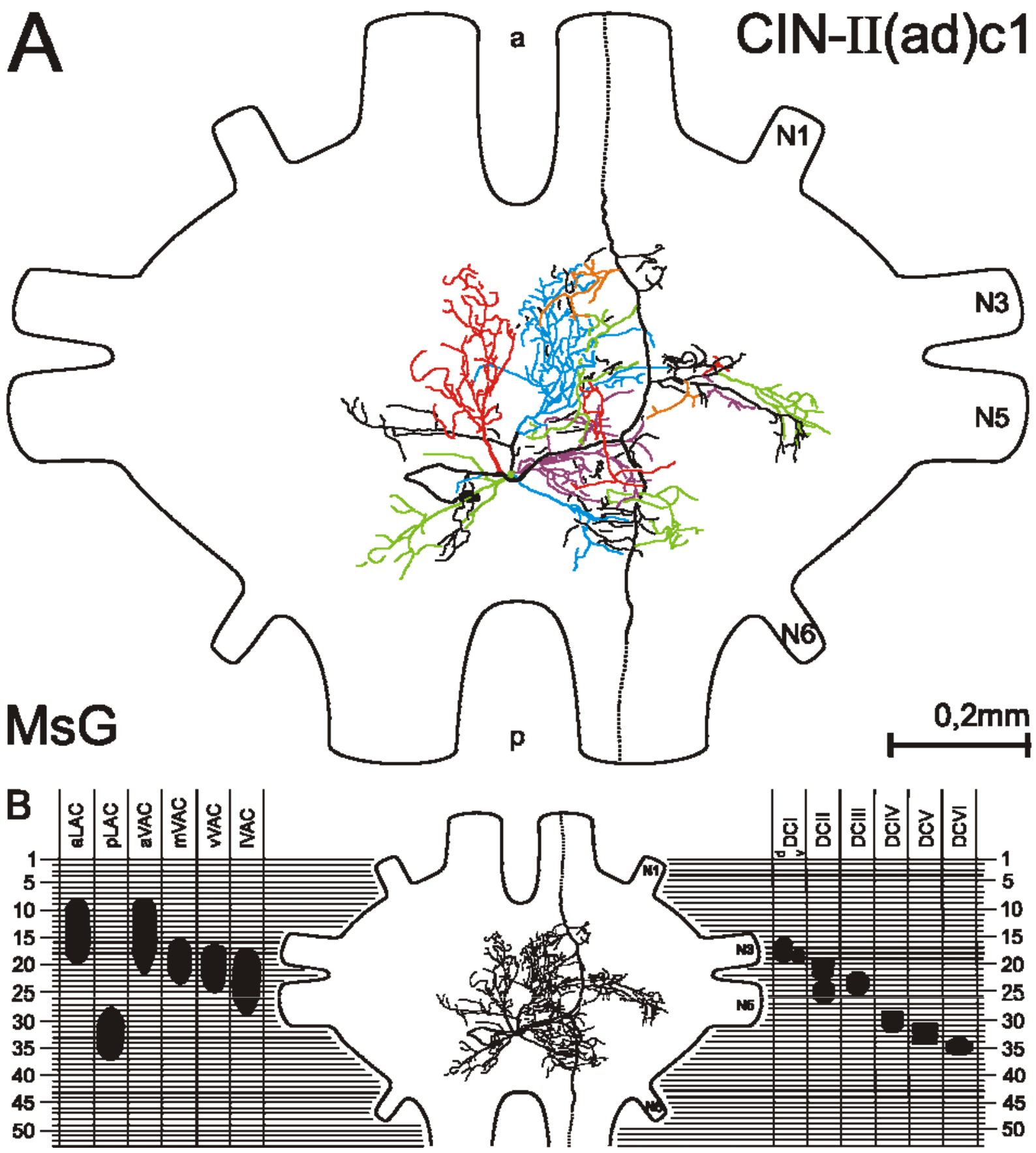

Abb. 22: Intersegmentales Interneuron CIN-II(ad)c1 mit medianem Hauptverzweigungsbereich (Typ II) und unilateral-axonaler Bifurkation. A: Gezeichnete 2D-Projektion, Mesothorakalganglion. Dorsal im kontralateralen (rechten) Konnektiv durchlaufendes Axon. Das Soma $(\varnothing=20 \mu \mathrm{m})$ liegt median-ventral auf der Höhe einer gedachten Vertikalen durch den inneren Randbereich der ipsilateralen Konnektive. Das Hauptverzweigungsareal erinnert an eine gewisse bilaterale Organisation, aber die Verzweigungen des Neuriten bleiben auf den medianen Bereich beschränkt. Die Hauptverzweigungen trennen sich ipsilateral vom primären Neuriten, der eine der hinteren dorsalen Kommissuren DCV oder DCVI passiert. Die Hauptausläufer verzweigen intermediär-dorsal, liegen ipsi- und kontralateral im näheren Umfeld der dorsalen Kommissuren und ziehen zum Teil über die Mittellinie hinweg. Sowohl auf- als auch absteigender Teil des Axons tragen einige zum Teil recht große Kollaterale, von welchen manche über einen weiten Bereich mit Fasern der medianen Hauptverzweigungen überlappen. Eine der großen Kollaterale (am aufsteigenden Axon-Teil) reicht unilateral bis an das laterale Neuropil pLAC heran. B: Übersicht desselben Neurons und Vergleich mit der relativen Lage wichtiger morphologischer Strukturen (aus: PFLÜGER et al. 1988; leicht modifiziert).

Abkürzungen: MsG: Mesothorakalganglion; a: anterior; p: posterior; N1-N6: Nerv1-6; Morphologische Abkürzungen in B Siehe Tabelle der verwendeten Abkürzungen. 

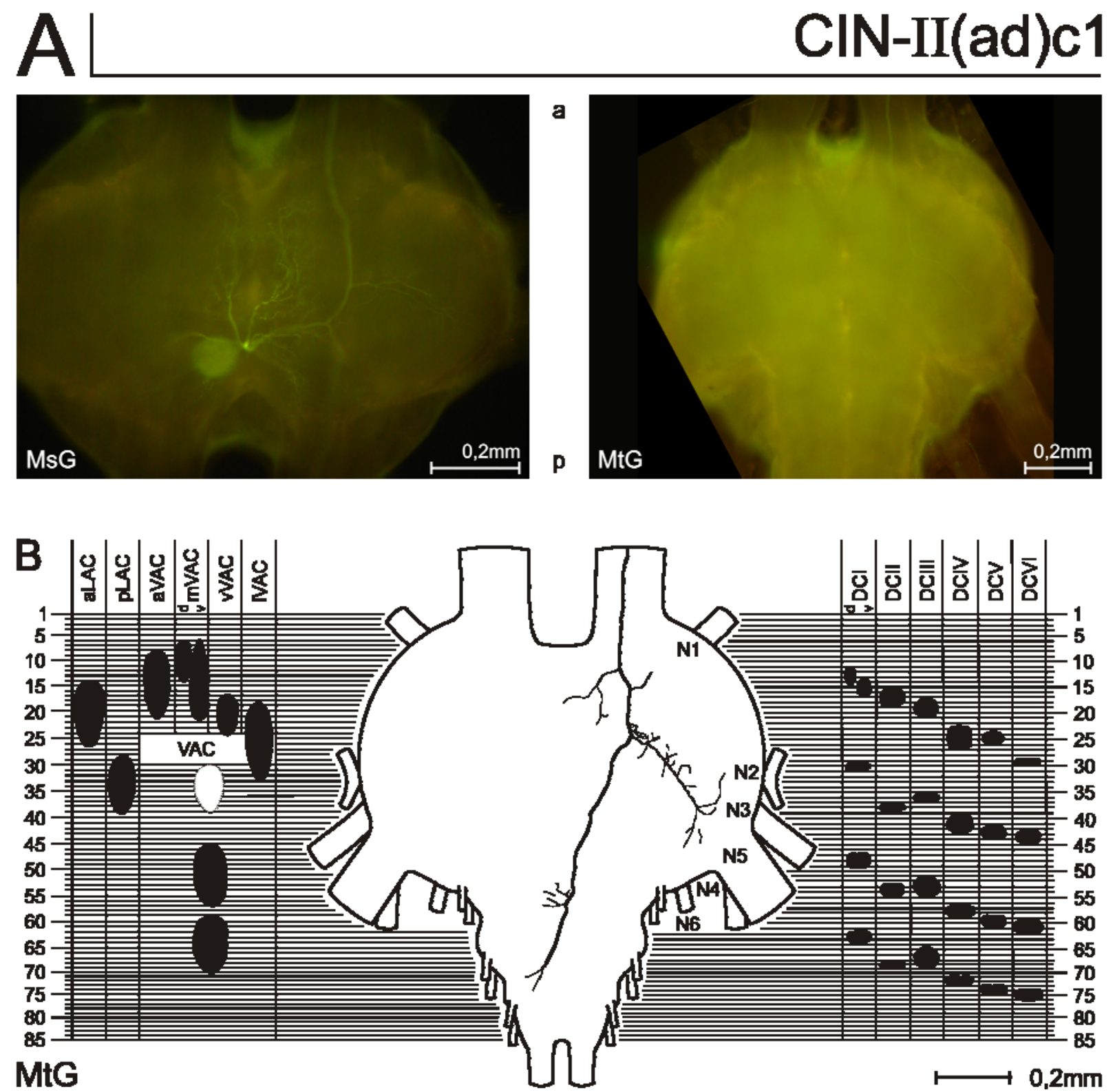

Abb. 23: A: Originalfotografien des Neurons CIN-II(ad)c1. MsG: Mesothorakalganglion, dorsale Aufsicht. $\mathrm{Zu}$ erkennen sind die medianen und $\mathrm{zu}$ beiden Seiten in der näheren Umgebung der dorsalen Kommissuren verzweigenden Hauptausläufer, die sich ipsilateral vom primären Neuriten, kurz vor dessen Durchtritt durch die Kommissur abtrennen. Das im kontralateralen Konnektiv durchlaufende Axon trägt am aufsteigenden Teil eine große Kollaterale, die dorsal verzweigt und bis weit nach lateral zieht. Das Soma liegt median-ventral in der Tiefe des linken Hemiganglions und ist außerhalb des Fokus getroffen. MtG: Metathorakalganglion. Der im kontralateralen (rechten) Konnektiv dorsal hereinziehende Teil des Axons ist bis zum Bereich der ersten Hauptverzweigung erkennbar. Diese größere Kollaterale (ebenfalls kontralateral) zieht weiter nach lateral und verzweigt dorsal bis intermediär auf Höhe der Nerven N3-N5. B: Gezeichnete 2D-Projektion der metathorakalen Strukturen des Neurons und Vergleich mit der relativen Lage wichtiger morphologischer Strukturen (aus: PFLÜGER et al. 1988; leicht modifiziert). Der Neurit zieht nach der ersten metathorakalen Hauptverzweigung zunächst nach posterior bis in die angelagerten Abdominalganglien hinein, nähert sich dabei zunehmend der Mittellinie an und verzweigt nach Passieren einer dorsalen Kommissur schließlich im ipsilateralen Hemiganglion weiter. Neben der großen Kollaterale, die im Bereich des lateralen Neuropils pLAC verzweigt, trägt das Axon anterior- und posterior-median noch mehrere kleine, zum Teil kommissurale Verzweigungen.

Abkürzungen: MsG: Mesothorakalganglion; MtG: Metathorakalganglion; a: anterior; p: posterior; N1N6: Nerv1-6; Abkürzungen in B Siehe Tabelle der verwendeten Abkürzungen. 
Hauptverzweigung zieht in den posterior-medianen Bereich bis zur Höhe des absteigenden Teils vom Axon. Ihre Verzweigungen sind größtenteils intermediär gelegen, steigen terminal zum Teil aber noch bis nach dorsal auf. Die fünfte Hauptverzweigung verläuft ipsilateral und verzweigt ebenfalls im posterior-medianen Feld. Sie teilt sich in einen Haupt- und zwei kleinere Seitenzweige. Der Hauptzweig zieht intermediär über das Soma hinweg und verzweigt etwa auf Höhe des zentralen Bereichs vom ipsilateralen Konnektiv. Der kommissurale Teil des verdickten primären Neuriten passiert eine der dorsalen Kommissuren DCV oder DCVI. Im Anschluss zieht er in lateraler Richtung und geht in das Axon über, das sich etwa auf mittlerer Konnektivhöhe (gedachte Vertikale durch die Mitte der sich ipsilateral gegenüberliegenden Konnektive) in eine auf- und eine absteigende Hauptkollaterale teilt. Beide verlaufen mehr oder weniger dorsal-median durch das Konnektiv und beide tragen mehrere dorsal verzweigende Seiten-Kollaterale, von welchen einige weiter nach lateral, andere zurück in Richtung Mittellinie ziehen. Die beiden größeren Kollaterale des absteigenden Axonteils verzweigen jeweils zu beiden Seiten dies und jenseits vom absteigenden Konnektiv, ändern dabei einmal bzw. mehrmals die Richtung und überlappen über einen weiten Bereich mit Fasern der Hauptverzweigungen zwei bis fünf. Die Kollaterale des aufsteigenden Axonteils ändern ihre Richtung nicht und verzweigen entweder weiter lateral oder ziehen in Richtung der Mittellinie, wo sie ebenfalls mit Fasern der Hauptverzweigungen zwei bis drei überlappen. Die größte Kollaterale zieht bis sehr weit nach lateral in Richtung des Nerven N5 und reicht an das posterior gelegene laterale Neuropil (pLAC) heran. Varikositäten sind an keiner der mesothorakalen Verzweigungen eindeutig erkennbar, was aber nicht bedeutet, dass es keine gibt. Die im mesothorakalen Ganglion ursprünglich fast vollständige Färbung war zum Zeitpunkt des Fotografierens schon etwas ausgeblichen.

\section{Metathorakalganglion:}

Das dorsal-median durch das kontralaterale (rechte) Konnektiv einlaufende Axon verzweigt sich im Metathorakalganglion über eine größere Strecke und reicht bis in die angelagerten Abdominalneuromere hinein (Abb. 23, B). Die Färbung ist im Metathorakalganglion nicht vollständig, so dass nicht alle Ausläufer bis zum Ende zu verfolgen sind und unklar ist, ob weitere hier nicht erkennbare Nebenverzweigungen vorhanden sind. Neben zwei kleineren Verzweigungen im anterioren Bereich trennt 
sich eine große Hauptverzweigung oberhalb des abgehenden Nerven N2 vom Neuriten und zieht lateral in Richtung von Nerv N5, wo sie im Bereich des posterior gelegenen lateralen Neuropils (pLAC) dorsal verzweigt. Der axonale Hauptausläufer nähert sich nach der Verzweigung weiterhin auf dorsalem Niveau zunehmend der Mittellinie. Etwa im zweiten angelagerten Abdominalneuromer trägt er mehrere kleine Seitenverzweigungen, die über dorsale Kommissuren hinweg in das ipsilaterale Hemiganglion hineinragen. Auch der Hauptausläufer selbst passiert ein kurzes Stück weiter eine dorsale Kommissur. Aufgrund seines eher schrägen Verlaufs bleibt aber unklar, um welche Kommissur es sich handeln könnte. Die dann folgende Verzweigungsstruktur lässt sich auf ispilateraler Seite nicht weiter verfolgen.

\section{Prothorakalganglion:}

Da die Fluoreszenz der neuronalen Verzweigungen im Prothorakalganglion zu schwach ist, können über diese Strukturen keine Aussagen gemacht werden.

Physiologie:

Das Neuron CIN-II(ad)c1 ist tonisch aktiv, bei Frequenzen zwischen 3-25Hz. Das Neuron reagiert mit phasischer Erregung bei dorsalen taktilen Tarsusreizen an beiden Vorder- und Mittelbeinen sowie auf taktile Reizung von am Thorax und Abdomen befindlichen Haaren. Am stärksten ist die Reaktion bei Reizung am rechten Vorderbein (kontralateraler Reiz), am linken Mittelbein (ipsilateraler Reiz) und von lateralen Haaren auf der Kutikula der ersten Abdominalsegmente (ipsi- und kontralateral). Der in Abbildung 24 unter A gezeigte ipsilaterale Reiz löst eine Reflexbewegung aus, während derer zunächst geringfügige Aktivität des ipsilateralen Depressors, im folgenden Verlauf aber synchron stärkere Muskelaktivität beider Depressoren erscheint. Erhöhte neuronale Aktivität erscheint kurz vor der Aktivität des kontralateralen Depressors, dauert dann synchron mit dieser an (Burst dauert etwas über $100 \mathrm{~ms}$ bei knapp $30 \mathrm{~Hz}$, umfasst also nur wenige Spikes), direkt gefolgt von einer vollständigen Hemmung für exakt die Dauer der Aktivität vom ipsilateralen Depressormuskel (Mittelbein links gereizt, Dauer der Aktivität ca. 150ms). Im Anschluss an die gehemmte Phase steigt die Neuronaktivität wieder an bis auf knapp $25 \mathrm{~Hz}$ und es folgt eine andauernde erhöhte Aktivität, die sich - langsam abfallend erst nach mehreren Sekunden wieder dem Niveau der Spontanfrequenz annähert. 


\section{CIN-II(ad)c1}

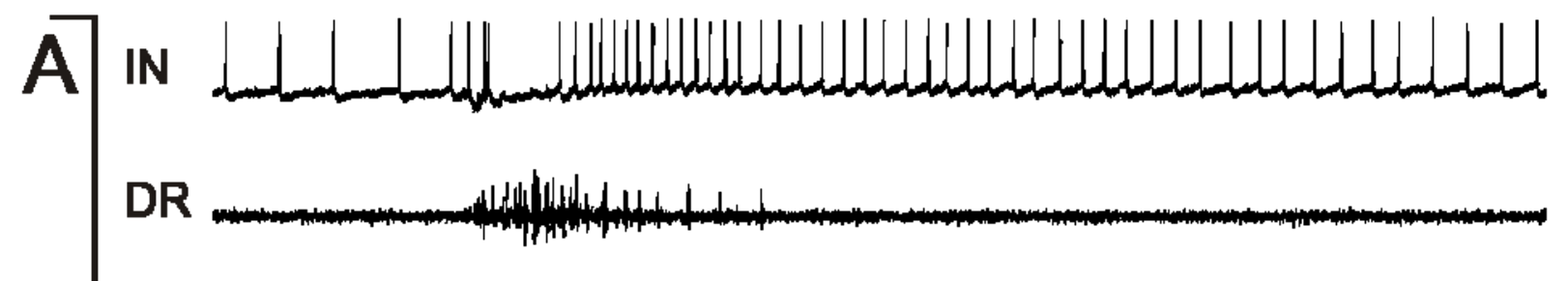

DL

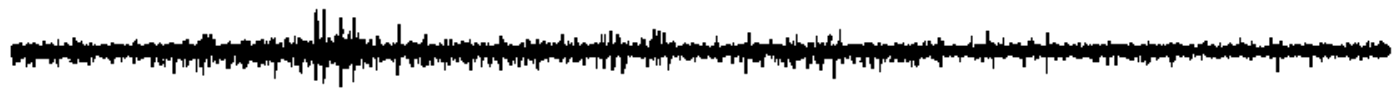

ST 几—-

30
$\mathrm{~Hz}$
0

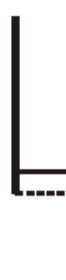

$B$

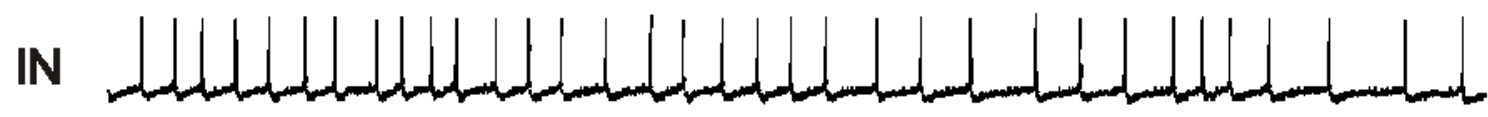

\section{DR}

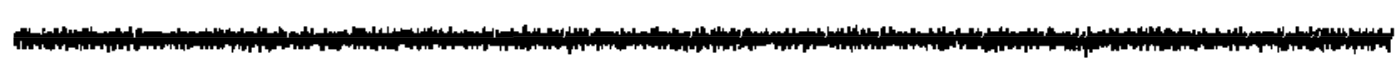

DL

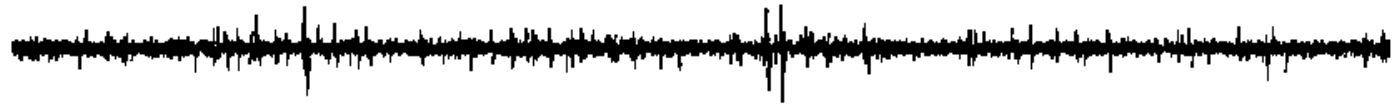

ST

ऍए

$\frac{9}{\frac{9}{2}}$

15
$\mathrm{~Hz}$
0

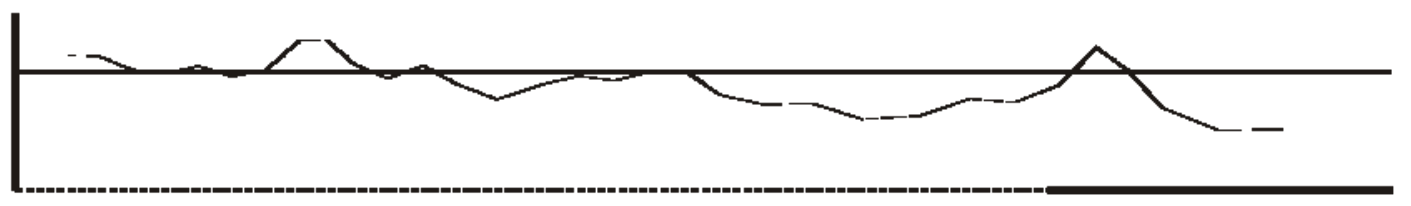

1s

Abb. 24: Aktivität, Instantan- und Spontanfrequenzen des Neurons CIN-II(ad)c1 und Muskelaktivitäten bei dorsal-taktilen Tarsusreizen. Das Neuron ist tonisch bei 3-25Hz aktiv. A: Ipsilateraler Reiz (Mittelbein links), Einmaliger dorsal-taktiler Tarsusreiz (kontinuierliche Reizung bis zum Reizerfolg, gestrichelte Linie in der ST-Spur): Hier wurde ein Reflex ausgelöst, bei dem zuerst der kontralaterale Depressor (DR) und unmittelbar darauf beide Depressoren gleichzeitig aktiv waren. Neuronale Aktivität (IN) erscheint zunächst kurz vor der Aktivität des kontralateralen Depressors und dauert dann synchron an. Für exakt die Dauer der darauf folgenden (erhöhten) Aktivität des ipsilateralen Depressors (DL) wird das Neuron gehemmt und zeigt im Anschluss wieder eine dauerhaft erhöhte, über mehrere Sekunden langsam abklingende Aktivität. B: Kontralateraler Reiz (Mittelbein rechts), dreifach dorsal-taktiler Tarsusreiz (kontinuierliche Reizung, gestrichelte Linie in der ST-Spur): Hier wurde die neuronale Aktivität nur unwesentlich durch die Reizungen beeinflusst. Die Instantanfrequenz steigt bei keiner Reizung signifikant über das Niveau der Spontanfrequenz. Nur nach dem ersten Reiz erscheint leicht erhöhte neuronale Aktivität synchron zur Aktivität des ipsilateralen Depressors. Bei den Folgereizungen ist keine klare Korrelation mehr erkennbar.

Abkürzungen: IN: Aktivität des Neurons; DR: Depressor trochanteris, rechtes Mittelbein; DL: Depressor tr., linkes Mittelbein; ST: dorsal-taktiler Tarsusreiz. 
Kontralaterale Reizung des Tarsus vom rechten Mittelbein beeinflusst die neuronale Aktivität wenn, dann nur schwach (Abbildung 24, B). Durch den ersten Reiz werden leichte, aber mehrere Sekunden andauernde Bewegungen des ipsilateralen Mittelbeins (linker Depressormuskel aktiv) ausgelöst. Da diese Bewegungen aber kaum sichtbar waren und sich die Aktivität des Neurons nur unwesentlich veränderte, wurde mehrfach gereizt. Nach jeder Reizung erscheint eine kurzzeitige Phase schwach erhöhter Neuronaktivität. Diese Phasen umfassen ebenfalls nur wenige Spikes und die Instantanfrequenz steigt dabei nur unwesentlich über das Niveau der Spontanfrequenz (maximal etwas über $10 \mathrm{~Hz}$ ). Aufgrund der auch zwischen den Reizungen kontinuierlich auftretenden schwachen Muskelaktivität (linker Depressor aktiv) ist die Korrelation zur neuronalen Aktivität nicht ganz klar. Eine Hemmung tritt bei dieser kontralateralen Reizung nicht auf.

Durch Hyper- und Depolarisation konnte die Neuronaktivität erwartungsgemäß beeinflusst werden, durch Depolarisieren konnte jedoch nicht erkennbar Muskelaktivität und damit Bewegung ausgelöst werden.

Kurzdiskussion:

Eine direkt bilateral die Extremitäten koordinierende Funktion ist bei Typ CIN-II Interneuronen aufgrund ihrer Morphologie nicht zu erwarten, denn sie verzweigen hauptsächlich meist dorsal-median und besitzen höchstens unilateral einen Nebenverzweigungsbereich. Das ist bei diesem Neuron CIN-II(ad)c1 der Fall und die große kontralaterale Kollaterale reicht sogar weit genug an das posterior gelegene laterale Neuropil (pLAC) heran, so dass das Neuron dort direkt auf Motoneurone verschalten könnte. Möglicherweise sind entlang der Fasern in diesem Bereich auch Varikositäten vorhanden, was weiterhin auf einen lokalen Projektionsbereich hinweisen würde. Die im Mesothorakalganglion fast vollständige Färbung war aber zum Zeitpunkt des Fotografierens bereits etwas ausgeblichen, so dass sich solche Strukturen nicht mehr zweifelsfrei erkennen lassen. Für eine direkt bilateral koordinierende Funktion des Neurons müsste es auch ipsilateral Verzweigungen im Bereich der dorsalen und ventralen Neuropile (vor allem IVAC, aLAC und pLAC) besitzen, wo sie bilateral relevante Informationen von ipsilateralen sensorischen Neuronen abgreifen könnten. Doch auf ipsilateraler Seite reichen die Verzweigungen des Neurons nicht weit nach lateral und sie sind auch eher intermediär bis dorsal 
gelegen. Es ist eine häufiger bei Interneuronen dieses Typs CIN-II wie auch bei diesem Neuron vorkommende Eigenschaft, dass ein Großteil der medianen Verzweigungen in der näheren Umgebung der dorsalen Kommissuren lokalisiert ist. Dies tritt häufig besonders ausgeprägt bei großen intersegmentalen Interneuronen auf, die ein im kontralateralen Konnektiv durchlaufendes Axon haben. Auch das Neuron CIN-II(ad)c1 ist im kontralateralen (rechten) Konnektiv durchlaufend, besitzt also auch noch Verzweigungen im Pro- und Metathorakalganglion. Es scheint eine allgemein die Extremitäten koordinierende Funktion zu haben (unabhängig davon, ob intersegmental und/oder bilateral). Das Neuron reagiert phasisch erregt auf taktile Tarsenreizung beider Mittelbeine und auch der Vorderbeine. Dass das Neuron bei Reizung des kontralateralen (rechten) Vorderbeins sogar noch eine höhere neurale Aktivität als bei taktiler Reizung an den Mittelbeintarsen zeigt, wirft die Frage auf, ob das im kontralateralen Konnektiv aufsteigende Axon im Prothorakalganglion neben einer mutmaßlichen Outputregion auch noch eine lokale Inputregion haben könnte. Außerdem reagiert es auch deutlich auf taktile Reizung von am Thorax befindlichen Haaren (ipsi- und kontralateral). Die phasischen Bursts erscheinen in jedem Fall immer im Zusammenhang mit der gemessenen Muskelaktivität (beider Depressoren der Mittelbeine), wenn auch die Korrelation nicht immer ganz klar ist. Eine ipsilaterale Tarsusreizung am linken Mittelbein löste einen Reflex aus, in dessen Verlauf zunächst leichte Aktivität des ipsilateralen Depressors und im Anschluss stärkere und mehr oder weniger synchrone Aktivität beider Depressoren auftrat. Erhöhte Neuronaktivität erschien erst unmittelbar vor dieser synchronen Muskelaktivität, also zeitlich kurz bevor der kontralaterale Depressor aktiv wurde. Das im weiteren Verlauf aufgetretene Aktivitätsmuster des Neurons ist allerdings komplizierter als man es erwarten würde, wenn das Neuron nur im Kontext eines bilateralen Reflexes direkt auf Motoneurone der kontralateralen Coxa-Depressoren verschaltet. Relativ genau für die Zeit der Aktivität des ipsilateralen Depressors wird das Neuron vollständig gehemmt (bei gleichzeitig andauernder Aktivität des kontralateralen Depressors) und im Anschluss erscheint wieder eine lang anhaltende erhöhte Neuronaktivität über mehrere Sekunden. Möglicherweise steuert das Neuron auf kontralateraler Seite auch einen anderen (antagonistischen) Muskel als den Depressor trochanteris an. Aufgrund der Datenlage und auch weil die Morphologie des Neurons im Prothorakalganglion nicht bekannt ist, lässt sich nichts Genaueres darüber aussagen, inwieweit im Kontext einer bei diesem Neuron anzunehmenden 
intersegmental koordinierenden Funktion auch noch eine bilaterale Koordination mit involviert ist. Große intersegmentale Interneurone mit einem Verzweigungsmuster, das dem hier beschriebenen Typ CIN-II entspricht, zeigen häufig ein komplexeres Aktivitätsmuster, dass zwar deutlich im Kontext der durch taktile Reizung ausgelösten Reflexe erscheint, bei welchem aber die Korrelation komplexer ist und oft nicht genau einem Ereignis (Aktivität eines bestimmten Muskels) zugeordnet werden kann. Bei solchen Neuronen fällt vor allem der schon eingangs beschriebene dorsal-mediane, manchmal nahezu ringförmige Verzweigungsbereich in der näheren Umgebung der dorsalen Kommissuren (ipsi- und kontralateral davon) auf. Möglicherweise ist dieser Bereich im Ganglion selbst eine Art zentrale „Schnittstelle“, in welcher Informationen über intersegmentale Koordination mit Informationen über bilaterale Koordination zusammenlaufen und damit untereinander koordiniert werden können. Ohne Kenntnis darüber, wie solche Neurone im Detail verschalten, lässt sich aufgrund ihres oft komplexen neuronalen Aktivitätsmusters kaum etwas über ihre Funktion aussagen.

\subsubsection{Median verzweigtes kommissurales Typ CIN-II Interneuron CIN-II(d)c1}

Morphologie:

\section{Mesothorakalganglion:}

Die Abbildungen 25-26 zeigen das intersegmentale Interneuron CIN-II(d)c1, das durch einen median bis anterior-median gelegenen Hauptverzweigungsbereich, sowie durch unilateral auftretende axonale Kollaterale gekennzeichnet ist. Der Hauptverzweigungsbereich ist in einen kontralateralen und einen ipsilateralen Teil unterteilt. Beide liegen in der näheren Umgebung der dorsalen Kommissuren DCIDCIV und verzweigen zum größten Teil dorsal im Bereich relativ zu den sich jeweils ipsi- und kontralateral gegenüber liegenden Konnektiven. Das Axon steigt kontralateral im linken Konnektiv ab und trägt eine große Kollaterale, die auf intermediärem Niveau nach lateral zieht. Der primäre Neurit durchquert wahrscheinlich die dorsale Kommissur DCl. Das Soma hat einen Durchmesser von etwa $20 \mu \mathrm{m}$ und liegt median-ventral im rechten Hemiganglion, etwa an der Position, wo sich die gedachte horizontale Linie durch die gegenüber liegenden Nerven 



Abb. 25: Intersegmentales Interneuron CIN-II(d)c1 mit medianem Hauptverzweigungsbereich (Typ II) und unilateralen axonalen Kollateralen. A: Gezeichnete 2D-Projektion, Mesothorakalganglion. Dorsal im kontralateralen (linken) Konnektiv absteigendes Axon. Das Soma $(\varnothing=20 \mu \mathrm{m})$ liegt medianventral im Bereich der inneren Randzone des Konnektivs. Der bogenförmig verlaufende primäre Neurit passiert die dorsale Kommissur DCl. Die Hauptverzweigungen trennen sich ipsi- und kontralateral vom primären Neuriten und tragen scheinbar keine Varikositäten. Sie verzweigen großteils dorsal und anterior-median und überlappen dabei untereinander. Der anfängliche Teil des kontralateral absteigenden Axons liegt intermediär, im späteren Verlauf im Konnektiv dorsal. Das Axon trägt neben einigen kleineren auch eine große Kollaterale, die intermediär nach lateral zieht und an das laterale Neuropil (pLAC) heranreicht. Die Verzweigungen der Kollaterale tragen überwiegend Varikositäten. B: Übersicht desselben Neurons und Vergleich mit der relativen Lage wichtiger morphologischer Strukturen (aus: PFLÜGER et al. 1988; leicht modifiziert).

Abkürzungen: MsG: Mesothorakalganglion; a: anterior; p: posterior; N1-N6: Nerv1-6; Kreuzschraffur in $\mathbf{B}$ : Bereich, in welchem überwiegend varikose Fasern (potentiell lokale Projektionsbereiche) vorkommen; Morphologische Abkürzungen in B Siehe Tabelle der verwendeten Abkürzungen. 

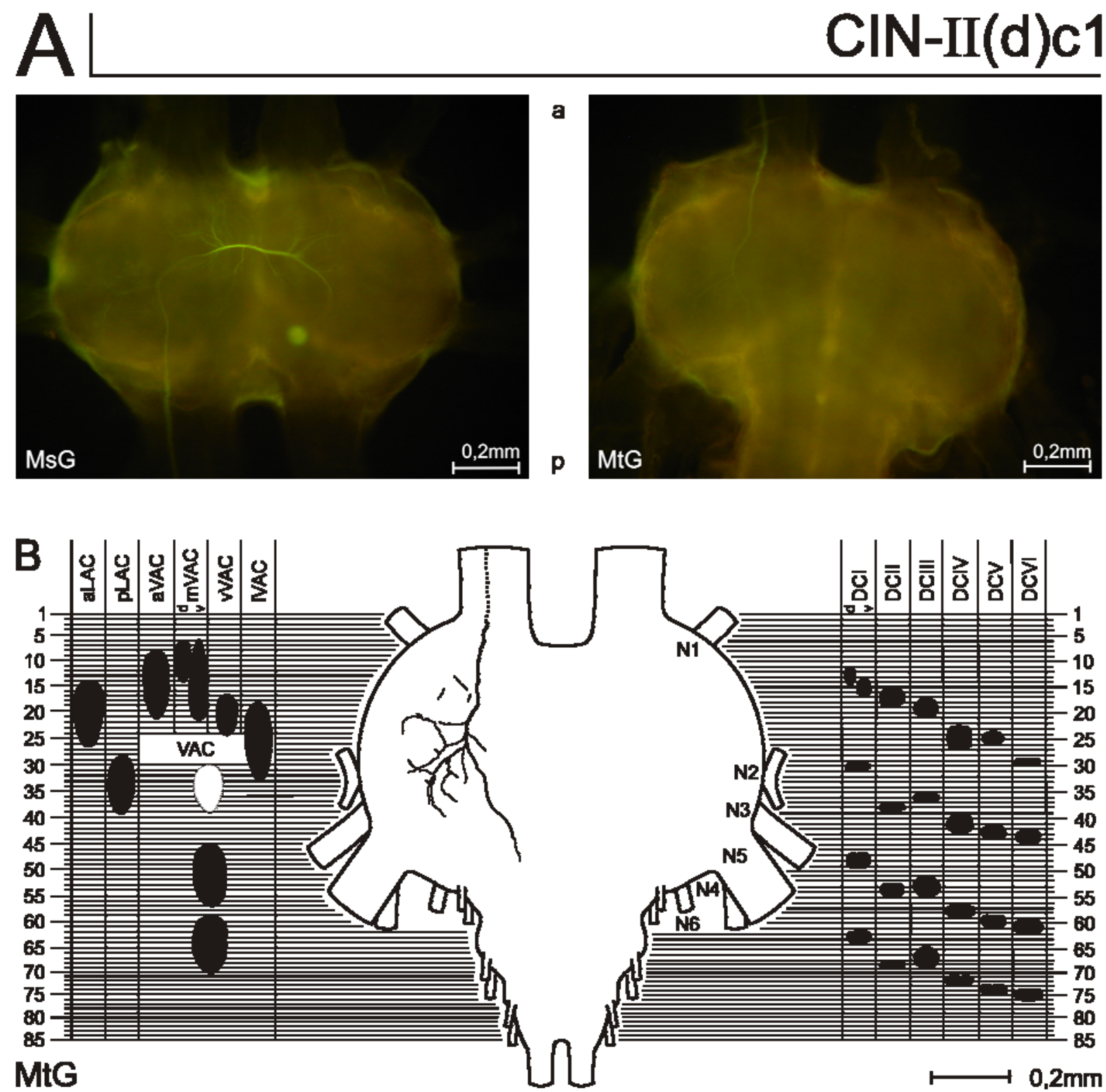

Abb. 26: A: Originalfotografien des Neurons CIN-II(d)c1. MsG: Mesothorakalganglion, intermediäre Fokal-Ebene. Zu erkennen sind die lateral gelegenen Ausläufer der medianen Hauptverzweigungen sowie die noch etwas tiefer im intermediären Bereich verzweigenden axonalen Kollaterale des kontralateralen Hemiganglions (schwach fluoreszierend). Das Axon steigt dorsal im Konnektiv ab und ist ebenfalls nicht ganz im Fokus getroffen. Das Soma liegt median-ventral in der Tiefe und nahe der inneren Randzone des ipsilateralen Konnektivs. MtG: Metathorakalganglion. Das dorsal durch das kontralaterale Konnektiv einlaufende Axon zieht im Metathorakalganglion nach posterior in den intermediären Bereich und verzweigt intermediär bis dorsal. Der genauere Verlauf ist im Einzelbild nicht gut zu erkennen. B: Gezeichnete 2D-Projektion der metathorakalen Strukturen des Neurons und Vergleich mit der relativen Lage wichtiger morphologischer Strukturen (aus: PFLÜGER et al. 1988; leicht modifiziert). Der Hauptverzweigungsbereich liegt im Metathorakalganglion im lateralen Bereich des kontralateralen Hemiganglions, zum Großteil auf Höhe der abgehenden Nerven N2-N5. Die Kollaterale verzweigen intermediär bis dorsal und könnten in die dorsalen lateralen Neuropile (aLAC, pLAC) hineinreichen. Aufgrund hier schwach ausgeprägter Färbung ist nicht zu erkennen, ob Varikositäten auftreten. Der Neurit setzt sich auf intermediärem Niveau möglicherweise weiter fort bis in die angelagerten Abdominalsegmente, jedoch lässt sich nicht mehr genau verfolgen, wie weit. Der gesamte Verzweigungsbereich scheint jedoch auf das kontralaterale Hemiganglion beschränkt zu sein. Abkürzungen: MsG: Mesothorakalganglion; MtG: Metathorakalganglion; a: anterior; p: posterior; N1-N6: Nerv1-6; Abkürzungen in B Siehe Tabelle der verwendeten Abkürzungen. 
N5 (posteriorer Randbereich) mit der gedachten Vertikalen durch die ipsilateralen Konnektive (innerer Randbereich) kreuzt. Der primäre Neurit zieht halbkreisförmig zunächst bis auf mittlere Konnektivhöhe und dann zurück in Richtung der Mittellinie. Dabei steigt er zunehmend bis nach dorsal an. Im intermediären Bereich trägt er mehrere kleine Seitenverzweigungen, die alle in laterale Richtung ziehen und intermediär bis etwa auf der Höhe relativ zum äußeren Randbereich der ipsilateralen Konnektive (gedachte Vertikale) verzweigen. Auf der Höhe relativ zum Nerv N3 beginnt der verdickte Teil des primären Neuriten, von dem alle Hauptverzweigungen abgehen. Die erste Hauptverzweigung zieht ein kurzes Stück (etwa $15 \mu \mathrm{m}$ ) zurück in laterale Richtung und teilt sich darauf in ein posterior und ein anterior gelegenes Verzweigungsfeld, die sich beide bis zum Bereich relativ zur lateralen Ausdehnung der ipsilateralen Konnektive erstrecken (gedachte longitudinale Verbindungslinie durch den lateralen Randbereich der Konnektive). Der anteriore Verzweigungsbereich teilt sich ein weiteres Mal in den größeren intermediär verzweigenden und einen bis nach dorsal ziehenden kleineren Ausläufer. Das posteriore Verzweigungsfeld erstreckt sich ausschließlich nach dorsal.

Am nächst folgenden Verzweigungspunkt liegen sich zwei mittelgroße Ausläufer gegenüber, die beide immer noch auf der Höhe relativ zu den Konnektiven intermediär bis dorsal verzweigen. Diese Anordnung von zwei sich mehr oder weniger direkt gegenüber liegenden mittelgroßen Nebenverzweigungen wiederholt sich kurz bevor der primäre Neurit die dorsale Kommissur DCI passiert. Der anteriore Ausläufer besitzt dorsale Verzweigungen, die sich von der näheren Umgebung der Mittellinie bis in den Bereich relativ zur inneren Randzone der ipsilateralen Konnektive erstrecken. Die posterior gelegene Verzweigung zieht schräg in Richtung des Somas und verzweigt ebenfalls dorsal im Bereich relativ zur Höhe der Konnektive (innere Randzone).

Im kommissuralen Bereich des primären Neuriten ziehen zwei kleine Seitenverzweigungen nach anterior. Eine von ihnen verläuft bogenförmig und zieht ein kurzes Stück über die Mittellinie hinaus in Richtung der dritten Hauptverzweigung. Letztere zieht, unmittelbar nachdem der primäre Neurit die Kommissur passiert hat, nach posterior und verzweigt dabei zunehmend dorsal im kontralateralen Hemiganglion. Ihre feineren Strukturen lassen sich nicht immer im Detail erkennen, da die Färbung in diesem Bereich nicht vollständig ist. Das kontralateral an den kommissuralen Teil des primären Neuriten anschließende Stück verläuft zunächst bis 
auf die Höhe relativ zur inneren Randzone der kontralateralen Konnektive, steigt noch weiter dorsal an und trägt mehrere kleine bis mittelgroße Nebenverzweigungen. Vier davon verlaufen nach anterior und verzweigen dorsal in der Nähe der Mittellinie. Eine andere Seitenverzweigung zieht nach posterior und verzweigt ebenfalls dorsal in der Nähe der Mittellinie.

Im unmittelbaren Anschluss an diese Nebenverzweigungen ziehen die Hauptverzweigungen drei bis fünf dicht hintereinander vom primären Neuriten ab, der sich seinerseits nun zunehmend intermediär verlagert. Dadurch steigen die weiter lateral abgehenden Verzweigungen auch aus dem intermediären Bereich empor. Die Hauptausläufer drei und vier verzweigen dorsal über ein weiteres Feld, das sich überwiegend anterior auf Höhe der kontralateralen, sich gegenüber liegenden Konnektive erstreckt. Die vierte Hauptverzweigung besitzt auch einen Ausläufer der nach posterior in den Bereich relativ zur inneren Randzone der kontralateralen Konnektive zieht. Sie verzweigt intermediär bis dorsal. Der vierten Hauptverzweigung liegt ein mittelgroßer Seitenast gegenüber, der nach posterior zieht und intermediär verzweigt.

Im anschließenden Abschnitt bleibt der primäre Neurit auch weiterhin verdickt. $\mathrm{Er}$ zieht auf intermediärem Niveau nach lateral in Richtung von Nerv N5. Auf der Höhe relativ zur lateralen Ausdehnung der Konnektive geht er bogenförmig in das absteigende Axon über, das neben mehreren kleinen auch eine große Kollaterale trägt. Diese verzweigt intermediär, zieht nach lateral und reicht bis an die lateralen Neuropilregionen (pLAC, aLAC) heran. Das Axon verläuft dorsal-median im kontralateralen Konnektiv. Die Verzweigungen der axonalen Kollaterale tragen überwiegend zahlreiche Varikositäten, im Gegensatz zu den eher dendritischen Fasern des medianen Hauptverzweigungsbereiches.

\section{Metathorakalganglion:}

Die axonalen Verzweigungen fluoreszieren aufgrund einer hier unvollständigen Färbung nur schwach (Abb. 26, A), so dass sich der Verlauf nicht bis zum terminalen Ende verfolgen lässt. So könnte sich der Neurit noch bis in die angelagerten Abdominalneuromere hinein erstrecken. Der Verzweigungsbereich scheint im Metathorakalganglion aber auf das kontralaterale Hemiganglion beschränkt zu sein (Abb. 26, B). Das Axon läuft dorsal-median über das kontralaterale (linke) Konnektiv ein und verbleibt auch im weiteren Verlauf im Ganglion selbst auf diesem Niveau. 
Neben einigen kleinen Nebenverzweigungen liegt der Hauptverzweigungsbereich in der lateralen Hälfte des Hemiganglions und besteht aus zwei größeren Ausläufern, die in Richtung der Nerven N2-N5 ziehen und an die lateralen Neuropile (pLAC, aLAC) heranreichen. Sie verlaufen zunächst dorsal und dringen weiter lateral auch bis in den intermediären Bereich vor. Aufgrund der schwachen Färbung ist nicht erkennbar, ob an den Verzweigungen Varikositäten vorhanden sind und ob es sich somit überwiegend um einen weiteren Projektionsbereich des Neurons im Metathorakalganglion handelt.

\section{Physiologie:}

Das Neuron CIN-II(d)c1 ist tonisch aktiv mit einer Spontanfrequenz, die zwischen 30$60 \mathrm{~Hz}$ liegt, meist bei knapp $50 \mathrm{~Hz}$. Das Neuron reagiert phasisch erregt mit kurzen Bursts (für meist $100-150$ ms bei bis zu $80-100 H z$ ) auf Reizung beider Vorder- und Mittelbeine (dorsale, taktile Tarsusreize und passives Anheben) sowie auf taktile Reizung von lateralen am Abdomen befindlichen Haaren. Ein kontralateraler Reiz am linken Mittelbein löst am Präparat meist einen bilateralen Reflex aus, bei dem zuerst Muskelaktivität vom kontralateralen Depressor trochanteris gemessen wird, dicht gefolgt von (oder nahezu synchron mit) Aktivität des ipsilateralen Depressors (Abb. 27, A). Da das Präparat zum Zeitpunkt der Messungen nicht mehr sehr vital ist, sind die ausgelösten Reflexe manchmal schwer sichtbar (Anheben des gereizten Mittelbeins kaum erkennbar). In der Muskelableitung des kontralateralen Depressors erscheint in jedem Burst meist auch ein Spike der fast-unit des Depressor trochanteris. In jedem Fall ist die neuronale phasische Erregung streng korreliert zu dieser Muskelaktivität des kontralateralen Depressors (linkes Mittelbein). Sie tritt in der Regel wenige Millisekunden früher auf, ist dann für die Dauer der Muskelaktivität synchron aktiv und überdauert diese meist sogar. Meist zeigt das Neuron diese phasische Erregung auch noch, wenn der ipsilaterale Depressor schon aktiv ist und in einigen Fällen dauert die neuronale Erregung noch bis zur Beendigung dieser Muskelaktivität an, jedoch nicht darüber hinaus. Reizung am kontralateralen (rechten) Mittelbein durch dorsal-taktile Tarsusreize beeinflusst die neuronale Aktivität auf vergleichbare Weise (nicht gezeigt). Jedoch führten die ausgelösten Reflexe nicht zum Auftreten von Muskelaktivität bei einer der beiden Depressormuskeln, so dass die Korrelation zur neuronalen Aktivität unklar ist. 


\section{$\mathrm{CIN}-\mathrm{II}(\mathrm{d}) \mathrm{c} 1$}

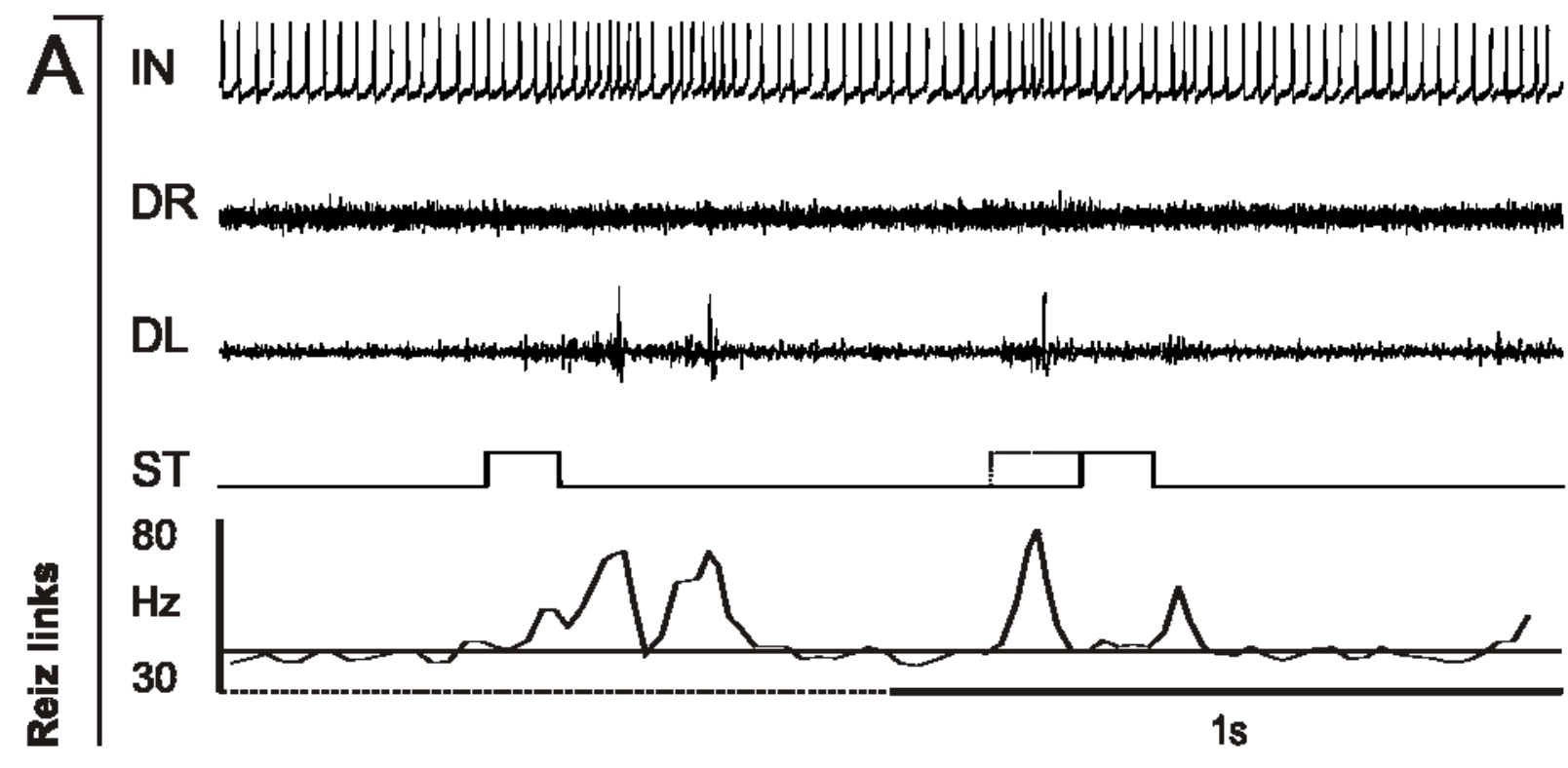

DR
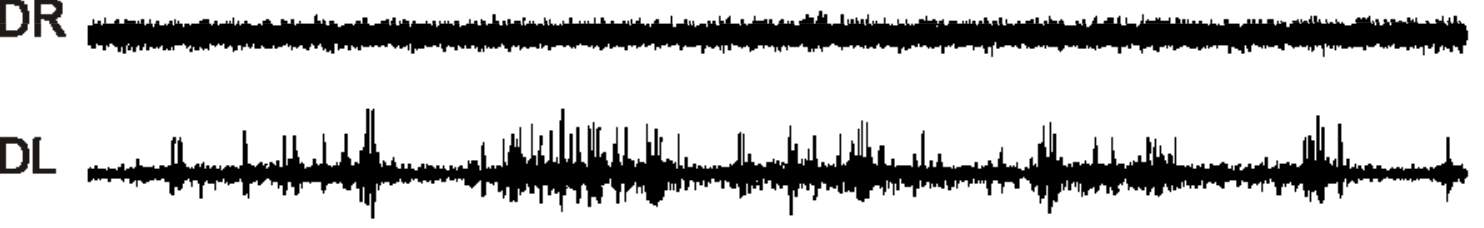

SP



Abb. 27: Aktivität, Instantan- und Spontanfrequenzen des Neurons CIN-II(d)c1 und Muskelaktivitäten bei kontralateraler Reizung (linkes Mittelbein). Das Neuron ist in Ruhe spontan bei etwa $45 \mathrm{~Hz}$ aktiv. A: Zweimaliger dorsal-taktiler Tarsusreiz (Reizindikation beim zweiten Reiz nicht zeitgenau, durch gestrichelte Linie in der ST-Spur gekennzeichnet). Die Reize lösen einzelne Zuckungen (einzelne große Spikes) der kontralateralen fast-unit vom Depressor trochanteris (DL), jedoch nur schwache Muskelaktivität des ipsilateralen Depressors (DR) aus. Dieser ist nach dem ersten Reiz synchron, nach dem zweiten etwas verzögert zur Aktivität des kontralateralen Depressors aktiv. In streng synchroner Korrelation zur Aktivität des kontralateralen Depressors erscheint neuronale Erregung (IN) immer einige Millisekunden vor Auftreten der Spikes der fast-unit und überdauert diese, jedoch nicht die Aktivität des ipsilateralen Depressors. B: Zweimaliges passives Anheben vom linken Mittelbein. Der hier ausgelöste Widerstandsreflex äußert sich durch kontinuierlich in mehreren Salven auftretende Spikes der fast-unit des kontralateralen Depressors (DL). Die neuronale Aktivität erscheint wie in A immer streng synchron korreliert zu dieser Muskelaktivität.

Abkürzungen: IN: Aktivität des Neurons; DR: Depressor trochanteris, rechtes Mittelbein; DL: Depressor tr., linkes Mittelbein; ST: dorsal-taktiler Tarsusreiz. 


\section{$\mathrm{CIN}-\mathrm{II}(\mathrm{d}) \mathrm{c} 1$}

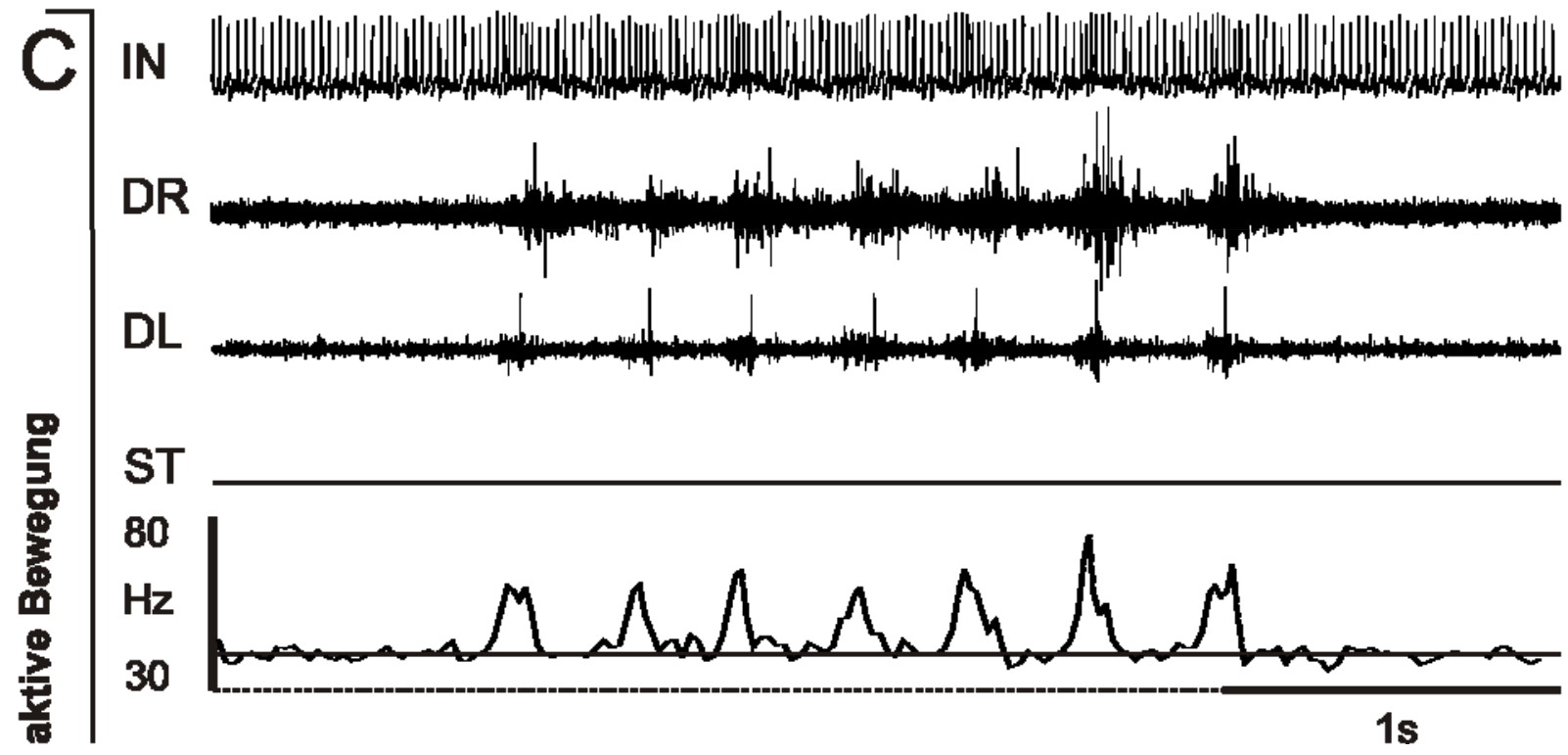

Abb. 28: C: Aktivität, Instantan- und Spontanfrequenzen des Neurons CIN-II(d)c1 und Muskelaktivitäten bei aktiver Bewegung beider Mittelbeine (nicht klar alternierende Korrelation, kein Laufen). Die neuronale Aktivität (IN) ist nach dem gleichen Prinzip wie in Abbildung 28 (A-B) gezeigt, immer streng synchron zur Aktivität des kontralateralen Depressormuskels (DL) korreliert und tritt auch ohne Reizung bei spontanen Bewegungen auf. Sie überdauert nicht die Aktivität des ipsilateralen Depressors (DR), die immer etwas verzögert zu der des kontralateralen Depressors erscheint.

Abkürzungen: IN: Aktivität des Neurons; DR: Depressor trochanteris, rechtes Mittelbein; DL: Depressor tr., linkes Mittelbein; ST: dorsal-taktiler Tarsusreiz.

Ein dauerhaftes passives Auslenken des ipsilateralen (linken) Mittelbeins löst einen Widerstandsreflex aus, bei welchem der ipsilaterale Depressor für die Dauer der Auslenkung dauerhaft aktiv ist und wo zahlreiche Spikes der fast-unit in mehreren Salven erscheinen (Abb. 27, B). Die neuronalen Bursts erscheinen auch bei diesem passiven Anheben und sind ebenfalls nach diesem Prinzip streng synchron korreliert zu dieser kontralateralen Muskelaktivität. Die Instantanfrequenz der Neuronaktivität liegt während der Auslenkbewegung teilweise auch bei etwas über $100 \mathrm{~Hz}$. Es zeigt sich dagegen kein erkennbarer Zusammenhang zwischen Auslenkamplitude und neuronaler Erregung. Der ipsilaterale Depressor scheint bei diesem lokalen Widerstandsreflex nicht mit involviert zu sein, denn es tritt in dieser Ableitung während dessen keine beachtliche Aktivität auf. Das ändert sich auch nicht bezüglich der Aufzeichnung der weiterhin synchron zur kontralateralen Muskelaktivität auftretenden neuronalen Aktivität während spontaner rhythmischer Muskelzuckungen (Abb. 28, C; Kein echtes Laufen, da die Vorderbeine zum Zeitpunkt dieser 
Sequenz inaktiv sind). Auch ohne Reizung erscheinen die neuronalen Bursts wieder nach gleichem Prinzip und Timing. Das Interneuron ist streng synchron zur kontralateralen Muskelaktivität korreliert. Die neuronalen Bursts beginnen gleichzeitig oder wenige Millisekunden früher als die Aktivität des kontralateralen Depressors und sind dann mindestens für die Dauer dieser Muskelaktivität präsent. Manchmal erscheint neuronale Erregung auch noch während ipsilateraler Muskelaktivität, aber sie überdauert diese nicht.

Eine Hemmung des Interneurons war im Laufe der Aufzeichnungen bei keiner der durchgeführten Reizungen zu beobachten. Hyper- und Depolarisation des Membranpotentials haben die zu erwartenden Effekte, es zeigt sich aber kein erkennbarer Einfluss auf eine der gemessenen Muskelaktivitäten.

Kurzdiskussion:

Die Funktion der intersegmentalen Koordination der Extremitäten ist bei diesem Neuron CIN-II(d)c1 sehr wahrscheinlich. Dafür spricht zum einen die streng synchrone Korrelation ausschließlich zur Aktivität des kontralateralen Depressors. Diese exakt synchrone Neuronaktivität erscheint auch während spontan aufgetretenen Bewegungen der Mittelbeine ohne Reizung. Aber auch morphologische Gegebenheiten sprechen für eine intersegmental koordinierende Funktion. Neben der unilateralen großen Kollaterale, die möglicherweise in den lateralen Neuropilregionen ( $\mathrm{pLAC}, \mathrm{aLAC}$ ) des kontralateralen Hemiganglions verzweigt und überwiegend Varikositäten aufweist, gibt es im Metathorakalganglion einen weiteren großen Verzweigungsbereich in der Region des lateralen Neuropils. Über eine potentielle Koordination des kontralateralen (linken) Hinterbeins kann jedoch nichts ausgesagt werden, da beide Hinterbeine bei dieser Präparation entfernt werden und da die Färbung im Metathorakalganglion auch nur schwach ausgeprägt ist. Varikositäten könnten auch hier an den axonalen Verzweigungen vorhanden sein, aber das lässt sich nicht eindeutig erkennen. Für eine intersegmental koordinierende Funktion spricht weiterhin die Tatsache, dass das Neuron neben der Reizung der Mittelbein-Tarsen auch noch auf Reizung beider Vorderbein-Tarsen und taktiler Haare am Thorax und Abdomen reagiert.

Wenn es sich bei dem unilateralen Verzweigungsbereich der Kollaterale im Mesothorakalganglion um einen lokalen Projektionsbereich handelt, so ist nicht zu 
erwarten, dass das Neuron direkt auf Motoneurone des kontralateralen Depressor trochanteris verschaltet. Zwar erscheint neuronale Erregung oft schon einige Millisekunden früher als diese Muskelaktivität, aber sie überdauert sie meistens noch. Bei einer direkten Verschaltung wäre bei andauerndem depolarisierenden Einfluss auch andauernde erhöhte Muskelaktivität zu erwarten. Depolarisieren des Membranpotentials hatte zwar den zu erwartenden Effekt auf die neuronale Aktivität, jedoch keine erkennbaren Auswirkungen auf eine der synchron gemessenen Muskelaktivitäten, was wiederum nicht für eine (direkt oder indirekt) erregende Verschaltung des Neurons auf Motoneurone spricht. Darüber hinaus verzweigt die Kollaterale ungewöhnlich tief im intermediären Bereich (wenn sie auch dennoch die laterale Neuropilregion noch erreichen könnte) und nicht extrem weit dorsal, wie das bei den meisten intersegmentalen und kommissuralen Interneuronen, die in der näheren Umgebung der lateralen Neuropile liegen, der Fall ist (sowohl bilateraler Typ CIN-I als auch median verzweigender Typ CIN-II). Dennoch ist die neuronale Erregung immer zeitlich ausschließlich mit der Muskelaktivität korreliert. Die Auslenkamplitude bei passivem Anheben vom kontralateralen Mittelbein hat keinen deutlich erkennbaren direkten Einfluss auf die Neuronaktivität. Der dabei ausgelöste Widerstandsreflex äußert sich aber durch dauerhafte, in der Stärke variierende Aktivität des kontralateralen Depressors. In Phasen stärkerer Muskelaktivität werden in den synchron erscheinenden neuronalen Bursts höhere Frequenzen erreicht. Eine direkte synaptische Verschaltung des Neurons auf andere Motoneurone, möglicherweise solche, die einen antagonistischen Muskel ansteuern (beispielsweise Trochanter-Levator), ist denkbar.

Eine bilateral die Mittelbeine koordinierende Funktion erscheint möglich, aber wenn, dann nur auf indirektem Wege und in den Kontext der intersegmentalen Koordination integriert. Das Interneuron hat nur einen unilateralen (Neben-)Verzweigungsbereich und reicht auf ipsilateraler Seite nicht bis an die lateralen Neuropile heran. Daher bedarf es für eine bilaterale Koordination zwischen beiden Mittelbeinen noch mindestens eines weiteren Interneurons. Es gibt aber ein weites median gelegenes Verzweigungsfeld, das sich auf Höhe der sich ipsi- und kontralateral gegenüber liegenden Konnektive und im Umfeld der dorsalen Kommissuren DCI-DCVI erstreckt, ein häufig auftretendes morphologisches Merkmal des Interneuron-Typs mit median gelegenem Hauptverzweigungsbereich (Typ CIN-II). Möglicherweise ist dies eine „Schnittstelle“ für Interneuronen, die in den Kontext der bilateralen und/oder 
intersegmentalen Koordination involviert sind.

Zusammenfassend lässt sich sagen, dass das Neuron CIN-II(d)c1 aufgrund der streng synchronen Korrelation zur Muskelaktivität sehr wahrscheinlich eine intersegmental die Extremitäten koordinierende Funktion hat, wenn auch anzunehmen ist, dass das Neuron nicht direkt auf Motoneurone des kontralateralen Depressor trochanteris verschaltet. Eine Koordination vom kontralateralen Mittel- und Hinterbein ist aufgrund der in den kontralateralen Hemiganglien vom Meso- und Metathorakalganglion gelegenen Verzweigungsbereiche mit zum Teil zahlreich vorkommenden Varikositäten (Mesothorakalganglion) zu erwarten. Vielleicht ist aber auch eine bilaterale Koordination beider Mittelbeine auf indirektem Wege über ein anderes nachgeschaltetes Interneuron und in einem komplexeren Kontext der Koordination der Extremitäten mit involviert.

\subsubsection{Median verzweigtes kommissurales Typ CIN-II Interneuron CIN-II(a)i1}

Morphologie:

Mesothorakalganglion:

Die Abbildungen 29-30 zeigen das intersegmentale Interneuron CIN-II(a)i1, das zwei mediane Hauptverzweigungsbereiche hat (Typ CIN-II). Beide Verzweigungsfelder (eines ipsi- und das andere kontralateral gelegen) reichen auf beiden Seiten von der näheren Umgebung der dorsalen Kommissuren DCI-DCVI bis auf die Höhe relativ zur lateralen Ausdehnung der jeweiligen sich ipsi- oder kontralateral gegenüberliegenden Konnektive. Sie liegen dorsal bis intermediär und zeichnen sich durch weitläufige feinste Verzweigungen aus, die von eher dendritischem Charakter sind (Varikositäten können vorhanden sein, lassen sich aber nicht eindeutig erkennen). Einige rekurrente Fasern verlaufen über die Mittellinie in das jeweils gegenüber liegende Hemiganglion, wahrscheinlich ebenfalls über dorsale Kommissuren. Das Axon weist einen auffälligen „Looping“-förmigen Verlauf auf. Dadurch besteht eine morphologische Ähnlichkeit zu einem Interneuron-Typ (Benennung nicht bekannt), für welchen diese Struktur als kennzeichnend beschrieben ist (WATSON and BURROWS 1983). Das Neuron CIN-II(a)i1 steigt im ipsilateralen (rechten) Konnektiv auf und trägt unilateral zwei größere Kollaterale, die aber nicht lateral verzweigen, 
sondern im Bereich des „Looping“-förmigen Verlaufs des Axons und weiter anterior davon dorsal oder unterhalb dieser axonalen Struktur im intermediären Bereich verzweigen.

Das größere Soma $(\varnothing=35 \mu \mathrm{m})$ liegt ventral und anterior nahe der Mittellinie. Der primäre Neurit verläuft zunächst parallel zur Mittellinie nach posterior und steigt nach etwa 30 $\mathrm{m}$ steil in dorsaler Richtung auf. Auf intermediärem Niveau verläuft er dann in einem Bogen zurück nach anterior, ebenfalls parallel zur Mittellinie. Die Position des Somas (anterior-ventral und nahe der Mittellinie) und der bis hier beschriebene Verlauf des primären Neuriten ähneln der Morphologie des von WATSON und BURROWS (1983) beschriebenen Interneurons. Bei letzterem verläuft der primäre Neurit innerhalb des T-Traktes (siehe Abb.4, Ausschnitt 21). Aufgrund der morphologischen Ähnlichkeit beider Neurone (zumindest hinsichtlich der SomaLokalisation und des anfänglichen Verlaufs des primären Neuriten) besteht die Möglichkeit, dass auch der primäre Neurit des Neurons CIN-II(a)i1 innerhalb des TTraktes verläuft.

Ziemlich genau oberhalb der Stelle, an welcher der primäre Neurit dem Soma entspringt, mündet er nach seinem bogenförmigen Verlauf auf intermediärem bis dorsalem Niveau in einen verdickten Abschnitt. Zum besseren Verständnis ist diese Umgebung in der Abbildung 29 (Abb. 29, A) im vergrößerten Ausschnitt gezeigt. Da der primäre Neurit genau unterhalb dieses verdickten Abschnitts in selbigen mündet, ist dies in der Zeichnung etwas schwierig zu erkennen. Der verdickte Prozess des primären Neuriten teilt sich seinerseits unmittelbar in eine nach kontralateral ziehende kommissurale und eine ipsilateral gelegene Hauptverzweigung. An dieser Stelle entspringt auch das nach lateral ziehende Axon. Die nach kontralateral ziehende Hauptverzweigung verläuft in Richtung der dorsalen Kommissuren DClDCII. Vor Durchquerung einer dieser beiden Kommissuren ziehen von ihr zwei kleinere Seitenverzweigungen nach anterior ab. Eine verzweigt ipsilateral und dorsal über dem ventralen Soma, die andere zieht zunächst nach anterior und verläuft dann dorsal über die Mittellinie in das kontralaterale Hemiganglion, wo sie anterior-dorsal mit lokalen Verzweigungen auf der Höhe relativ zum inneren Randbereich der kontralateralen Konnektive (gedachte Verbindungslinie) überlappt. Auch kontralateral trägt der kommissurale Prozess des primären Neuriten zwei weitere kleine Seitenverzweigungen, die nach anterior ziehen, aber deren weiterer Verlauf in der Färbung nicht erkennbar ist. Nach der Durchquerung der Kommissur zieht der Neurit 

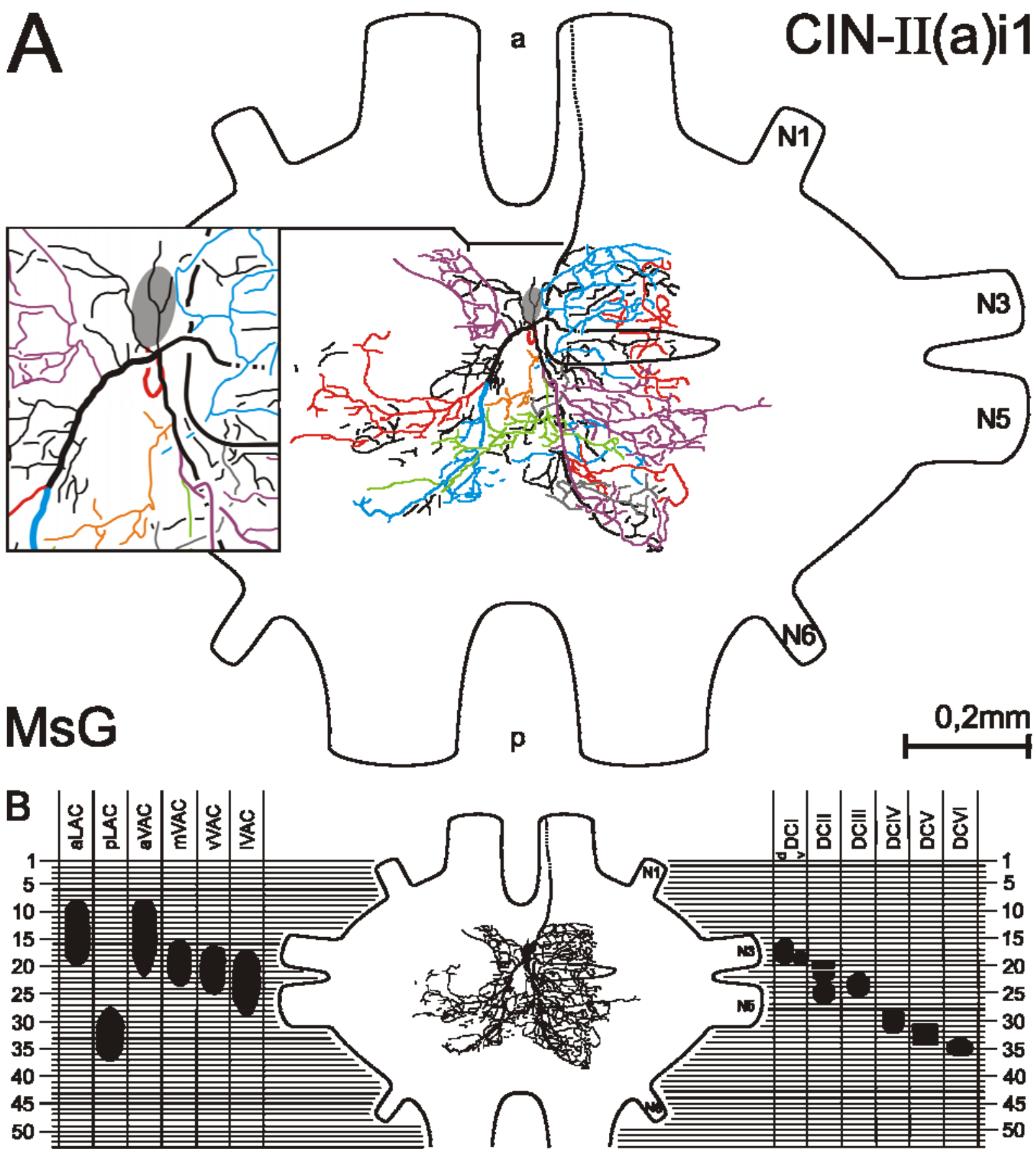

Abb. 29: Intersegmentales Interneuron CIN-II(a)i1 mit einem Hauptverzweigungsbereich im medianen Feld (Typ II). A: Gezeichnete 2D-Projektion, Mesothorakalganglion. Intermediär im ipsilateralen (rechten) Konnektiv aufsteigendes Axon. Das Soma $(\varnothing=35 \mu \mathrm{m})$ liegt anterior-ventral nahe der Mittellinie. Der primäre Neurit steigt (möglicherweise im T-Trakt) bogenförmig bis nach intermediär auf und mündet - fast oberhalb des Somas gelegen - in einen verdickten Prozess, welcher auf einer Seite über eine der dorsalen Kommissuren DCl oder DCII zur kontralateralen Hauptverzweigung führt, die einer ipsilateral gelegenen von optisch ähnlicher Gestalt gegenüber liegt. Beide Äste verzweigen dorsal bis intermediär zu beiden Seiten der dorsalen Kommissuren DCI-DCVI auf der Höhe relativ zu den Konnektiven. Das Axon verläuft in einem großen „Looping“-förmigen Bogen zunächst nach lateral, dann zurück zur Mittellinie und von dort in das ipsilaterale Konnektiv. Es besitzt zwei große Kollaterale, die intermediär und anterior vom „Looping“ gelegen verzweigen. Das Neuron hat eine morphologisch ähnliche Gestalt zu einem Interneuron-Typ mit charakteristischem "Looping" (WATSON and BURROWS 1983). B: Übersicht desselben Neurons und Vergleich mit der relativen Lage wichtiger morphologischer Strukturen (aus: PFLÜGER et al. 1988; leicht modifiziert).

Abkürzungen: MsG: Mesothorakalganglion; a: anterior; p: posterior; N1-N6: Nerv1-6; Morphologische Abkürzungen in B Siehe Tabelle der verwendeten Abkürzungen. 


\section{CIN-II(a)i1}

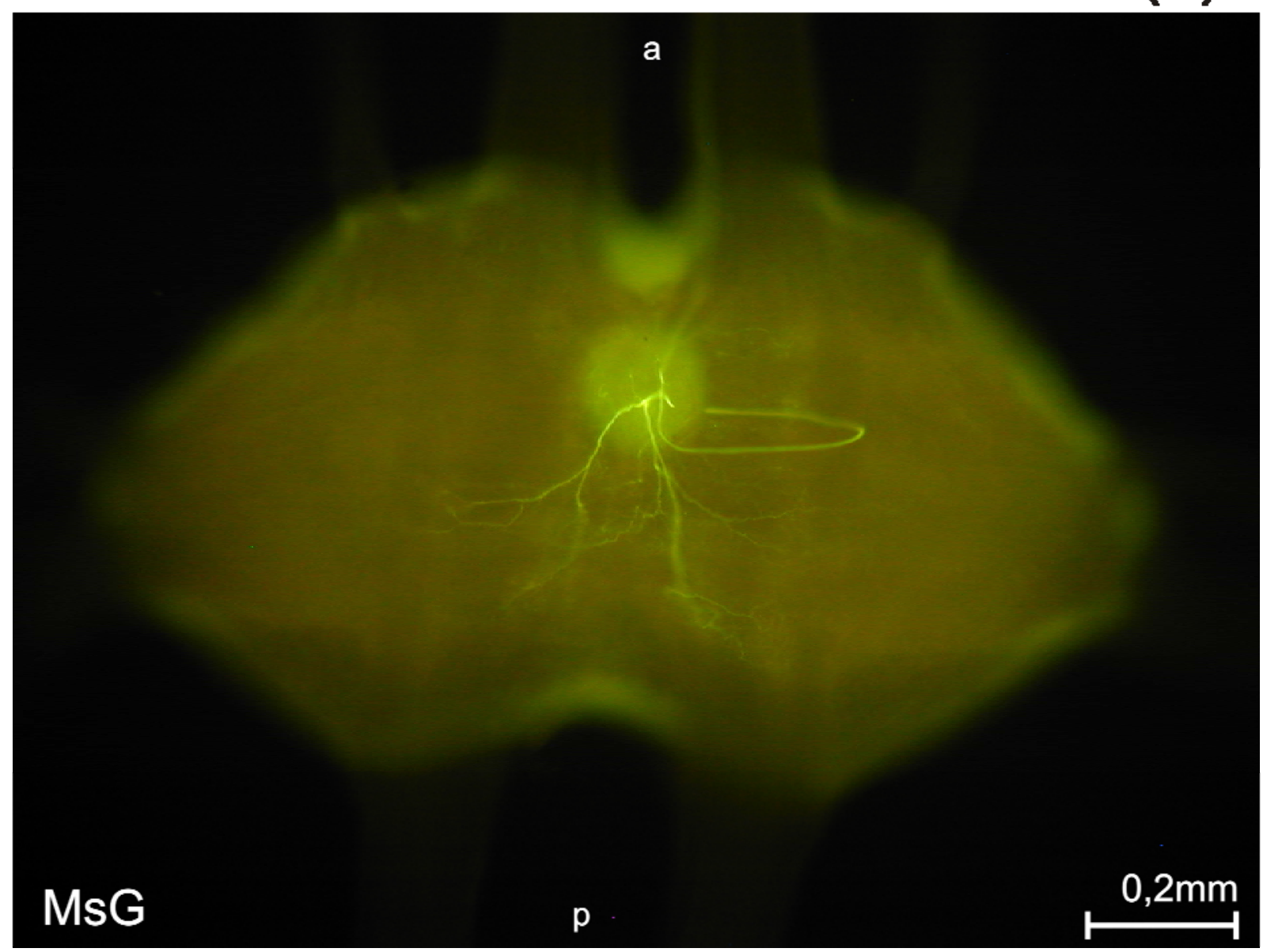

Abb. 30: Originalfotografie des Neurons CIN-II(a)i1, Mesothorakalganglion. Die Fokalebene liegt im dorsalen Bereich und lässt den kommissuralen Teil des Neuriten, den charakteristischen "Looping“förmigen Verlauf des Axons sowie den kontralateralen und ipsilateralen Verzweigungsbereich erkennen. Letztere beiden liegen jeweils in der näheren Umgebung der dorsalen Kommissuren DClDCVI und es gibt einige Seitenverzweigungen, die ebenfalls über dorsale Kommissuren oder kommissurale Trakte in das jeweils gegenüberliegende Hemiganglion hineinziehen und dort mit lokalen Verzweigungen zum Teil großflächig überlappen. Das Soma liegt anterior-ventral nahe der Mittellinie und fluoresziert diffus in der Tiefe des Ganglions. Das Axon steigt intermediär im ipsilateralen Konnektiv auf und ist ebenfalls außerhalb des fokalen Bereiches getroffen. An keiner der Verzweigungen lassen sich eindeutig Varikositäten erkennen, sie könnten aber dennoch vorhanden sein. In jedem Falle erscheinen alle Verzweigungen mehr oder weniger homogen, es gibt keine klar getrennten dendritischen oder varikosen Verzweigungsbereiche und auch keine deutlichen Unterschiede in der Struktur. Die Verzweigungen des primären Neuriten überlappen teilweise geringfügig mit solchen von Kollateralen.

Das Neuron besitzt eine morphologische Ähnlichkeit zu einem beschriebenen Interneuron-Typ, mit charakteristischem „Looping"-förmigen Verlauf des Axons (WATSON and BURROWS 1983).

Abkürzungen: MsG: Mesothorakalganglion; a: anterior; p: posterior.

bogenförmig nach posterior und verläuft dabei in der näheren Umgebung der dorsalen Kommissuren DCI-DCVI.

Die erste größere Unterverzweigung trennt sich unweit der Kommissur vom Neuriten und zieht nach anterior in den Bereich relativ zur inneren Randzone der 
kontralateralen Konnektive (gedachte Verbindungslinie).

Sie verzweigt zunächst dorsal und weiter anterior dann zunehmend intermediär. Im weiteren Verlauf der kontralateralen Hauptverzweigung auf der Höhe zwischen Nerv N3 und Nerv N5 entspringen mehrere kleine Nebenverzweigungen und verlaufen intermediär sowohl im Bereich relativ zur inneren Randzone der kontralateralen Konnektive, als auch näher zur Mittellinie hin. Es gibt aber keine in der Färbung erkennbare kontralaterale Verzweigung, die über die Mittellinie hinweg zurück nach ipsilateral zieht. Auf der Höhe relativ zur gedachten Horizontalen, die durch die Stelle zwischen Nerv N3 zu Nerv N5 verläuft, teilt sich der kontralaterale Neurit in zwei weitere Verzweigungen, von welchen eine nach lateral in Richtung von Nerv N5 und die andere etwas weiter posterior in Richtung von Nerv N6 verläuft. Beide ziehen nach dem Abzweig vom Neuriten zunächst auf dorsalem Niveau, verlagern sich im späteren Verlauf jedoch zunehmend nach intermediär. Der posteriore Ausläufer verzweigt auf der Höhe relativ zur lateralen Ausdehnung der kontralateralen Konnektive, die terminalen Endigungen des lateralen Ausläufers ziehen zum Teil auch etwas darüber hinaus, dürften jedoch ebenfalls nicht bis zum lateralen Neuropil (pLAC) vorstoßen.

Die ipsilaterale Hauptverzweigung zieht nach posterior und weist eine gewisse morphologisch-optische Ähnlichkeit zur kontralateral gelegenen auf. Auch ihre Nebenverzweigungen erstrecken sich größtenteils von der näheren Umgebung der dorsalen Kommissuren bis knapp über den Bereich relativ zur lateralen Ausdehnung der ipsilateralen Konnektive (gedachte Verbindungslinie) hinaus.

Die erste kleinere Nebenverzweigung verläuft schräg nach posterior und über die Mittellinie hinweg. Sie reicht knapp an lokale Verzweigungen der kontralateralen Hauptverzweigung heran und verläuft dorsal.

Auf der Höhe des Übergangsbereiches vom Nerv N3 zum Nerv N5 teilt sich der ipsilaterale Neurit in zwei jeweils über einen weiten Bereich verzweigende Felder. Eines liegt hauptsächlich dorsal bis intermediär, das andere darunter im intermediären Bereich. Die Färbung ist in diesem Abschnitt nicht vollständig und die Verzweigungen größtenteils sehr fein, so dass sie sich oft nicht durchgängig verfolgen lassen. Der tiefer liegende intermediäre Ausläufer ist weniger komplex als der dorsale aufgebaut und trägt weniger Unterverzweigungen. Er zieht zunächst nach posterior und verzweigt dann im späteren Verlauf größtenteils zunehmend lateral ohne aber den Bereich relativ zur äußeren Randzone der ipsilateralen 
Konnektive zu überschreiten. Zwei kleinere Unterverzweigungen ziehen in Richtung der Mittellinie, eine davon auch darüber hinweg bis in das kontralaterale Hemiganglion. Der darüber liegende intermediär-dorsale Ausläufer teilt sich kurz nach seinem Abzweig von der ipsilateralen Hauptverzweigung in zwei Unterverzweigungen. Eine davon zieht unweit der Mittellinie nach posterior und teilt sich abermals in zwei Ausläufer. Der erste Ausläufer zieht ipsilateral in Richtung des Nerven N5 und verzweigt dorsal auf der Höhe einer gedachten Vertikalen, die zentral durch die ipsilateralen Konnektive verläuft. Der andere Ausläufer zieht über die Mittellinie hinweg in Richtung des kontralateralen Nerven N5 und verzweigt ebenfalls hauptsächlich dorsal auf der Höhe relativ zu den kontralateralen Konnektiven (gedachte longitudinale Verbindungslinie durch deren inneren Randbereich). Es kommt hier teilweise zur Überlappung mit lokalen terminalen Verzweigungen der kontralateralen Hauptverzweigung.

Die andere dorsale Unterverzweigung des ipsilateralen Hauptverzweigungsfeldes hat weitverzweigte feinste Fasern und deckt ein großes posterior vom „Looping“förmigen Axon gelegenes Feld ab. Sie besteht aus zwei größeren Ausläufern, von welchen einer mehr posterior und intermediär, der andere mehr lateral und dorsal verzweigt. Letzterer hat eine einzelne Faser, die noch etwas weiter nach lateral verläuft. Aber es ist nicht erkennbar, ob sie sich noch bis zum lateralen Neuropil (pLAC) erstreckt.

Das Axon verläuft zunächst auf Höhe des Nerven N3 nach lateral und dann in einem nach dorsal verlaufenden großen „Looping“-förmigen Bogen wieder zurück zur Mittellinie. Von dort zieht es nach anterior, verlagert sich dabei zunehmend intermediär und steigt schließlich ipsilateral und intermediär am inneren Rand des Konnektivs entlang auf. Es gibt mehrere axonale Kollaterale, unter anderem zwei weitverzweigte, die im Umfeld des „Looping“-förmigen Abschnitts vom Axon oder anterior davon liegen und dorsal, aber auch unterhalb dieser axonalen Struktur im intermediären Bereich verzweigen. Die erste große Kollaterale verlässt das Axon etwa mittig am dorsalen Teil des „Loopings“, die zweite auf Höhe des Somas, in dem Bereich, wo das Axon nach anterior in Richtung des Konnektivs zieht. Auch in diesem Abschnitt lassen sich die feinen Verzweigungen nicht durchgängig verfolgen. Es gibt möglicherweise noch mehr als zwei große Kollaterale.

In beiden Hemiganglien erstrecken sich die Verzweigungen zum größten Teil dorsal bis intermediär, auf der Höhe relativ zu den sich jeweils ipsi- und kontralateral gegen- 
überliegenden Konnektiven. Als morphologische Referenzstrukturen in der näheren Umgebung sind vor allem die intermediären (DIT, VIT), der jeweilige laterale (LDT) und dorsal-mediane Trakt (DMT) in Betracht zu ziehen. Keine der Verzweigungen reicht weit nach lateral in die Region der dorsalen lateralen Neuropile (aLAC, pLAC). In der Färbung lassen sich an keiner der Verzweigungen oder Kollaterale eindeutig Varikositäten erkennen.

\section{Prothorakalganglion:}

Aufgrund unvollständiger Färbung ist über die Verzweigungstruktur des Neurons CIN-II(a)i1 im Prothorakalganglion nichts bekannt.

Physiologie:

Da sich am Präparat Reflexe häufig kaum sichtbar auslösen ließen, wurde jeweils kontinuierlich gereizt bis zum Reizerfolg (repetitive taktile Tarsusreize bis zum sichtbar ausgelösten Reflex). In den Abbildungen 31-32 ist der jeweilige Zeitraum solcher kontinuierlichen Reizungen durch eine gestrichelte Linie in der Spur der Reizindikation (ST) angegeben. Der Reizpuls selbst markiert den jeweiligen Beginn der kontinuierlichen Reizung.

Abbildung 31 (A-B) zeigt die nach kontralateraler Tarsusreizung am linken Mittelbein aufgetretenen bilateralen Reflexbewegungen, bei welchen beide Depressoren der Mittelbeine mehr oder weniger synchron aktiv sind. Erhöhte Muskelaktivität des ipsilateralen Depressors (erkennbar am Auftreten von Spikes der jeweiligen fast-unit innerhalb der Bursts) erscheint nur wenige Millisekunden verzögert zur kontralateralen. Das Neuron CIN-II(a)i1 reagiert während drei Sequenzen taktiler Tarsusreizung am kontralateralen Mittelbein nicht immer in exakt der gleichen Weise. In der Abbildung 31 A (kontinuierliche Reizung löst zweimal einen Reflex aus) ist zu erkennen, dass Phasen der neuronalen Erregung zeitlich kurz vor und nach der synchronen stärkeren Muskelaktivität auftreten. Die präaktive Phase der neuronalen Erregung dauert noch wenige Millisekunden synchron zur Aktivität beider Depressoren an, gefolgt von einer Phase in der das Neuron für die weitere Dauer dieser synchronen stärkeren Muskelaktivität gehemmt wird. Die Hemmung ist aber nicht vollständig, das Neuron ist in dieser Phase nicht inaktiv. Einige Millisekunden bevor die Muskelaktivität beendet ist, setzt wieder eine kurze Phase schwacher 


\section{CIN-II(a)i1}

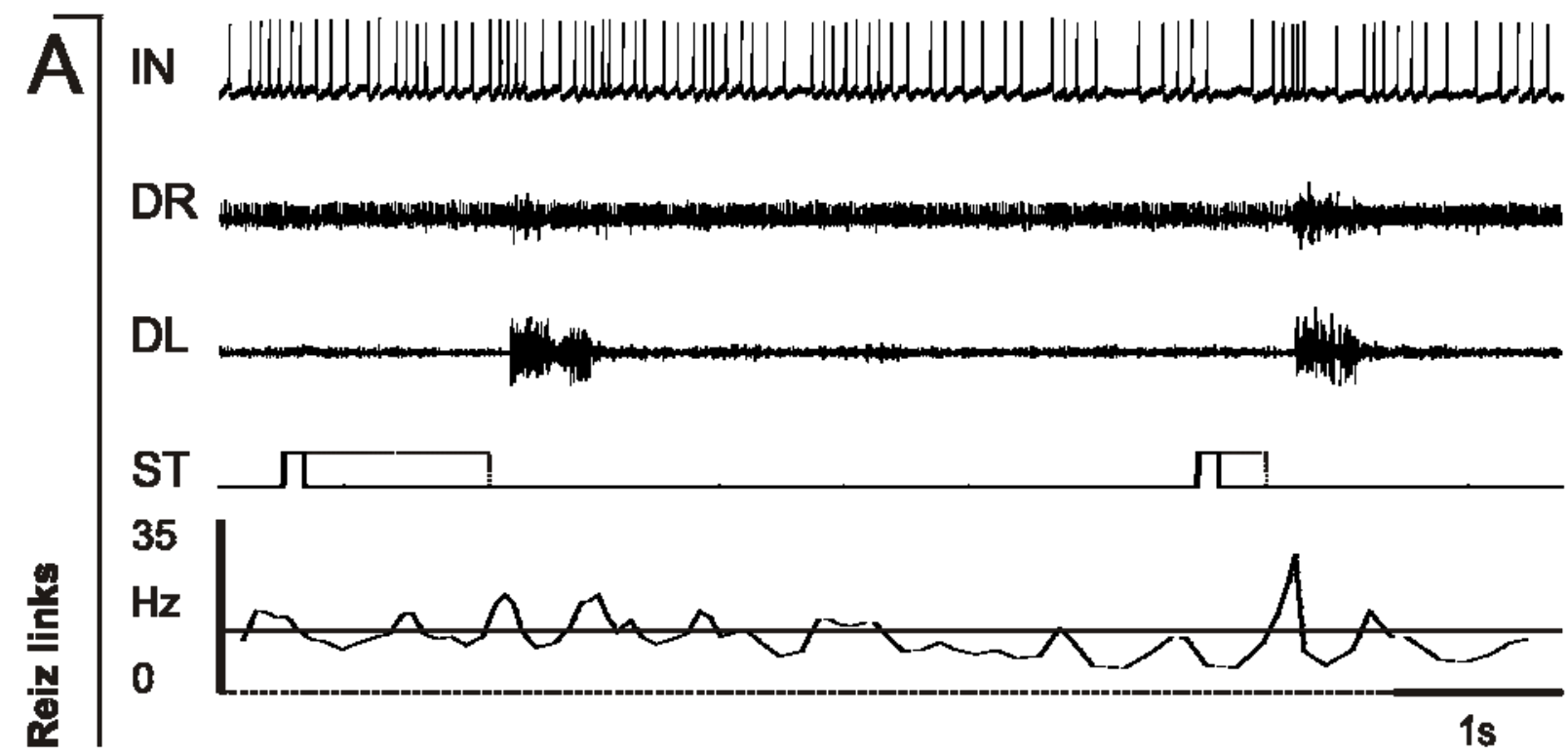

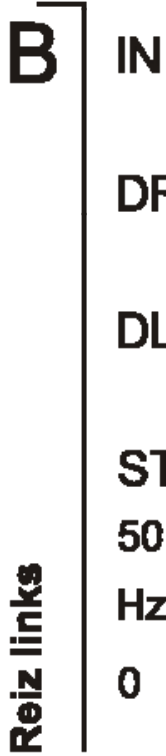

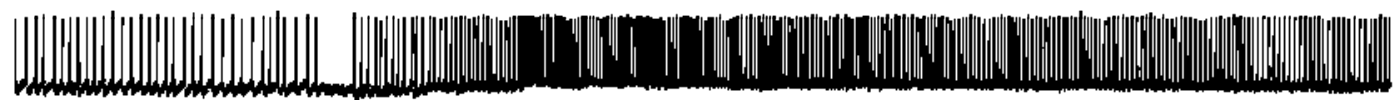

DR

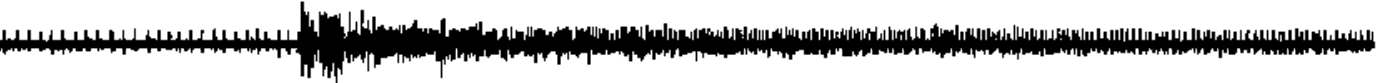

$\mathrm{DL}$

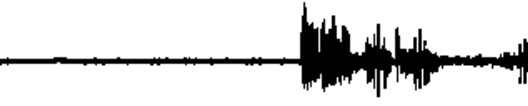

ST
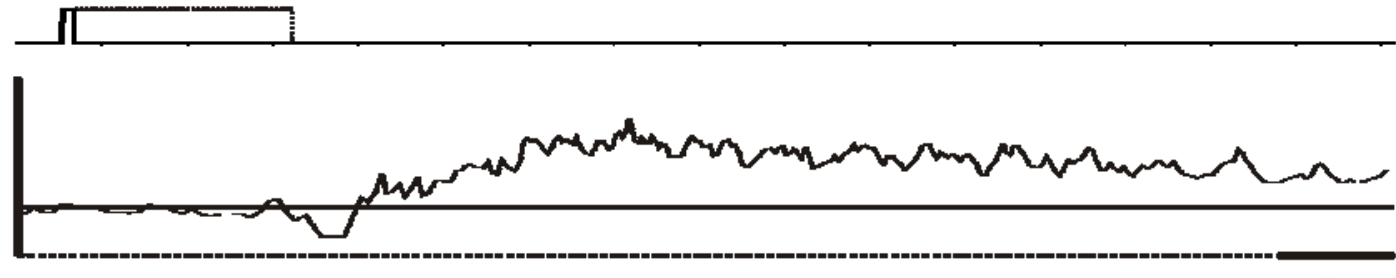

Abb. 31: Aktivität, Instantan- und Spontanfrequenzen des Neurons CIN-II(a)i1 und Muskelaktivitäten bei kontralateraler Reizung (linkes Mittelbein). Das Neuron ist tonisch aktiv bei etwa 15Hz. A: Zweifacher dorsal-taktiler Tarsusreiz (kontinuierliche Reizung bis zum Reizerfolg, gestrichelte Linie in der ST-Spur). Hier wird jeweils ein Reflex ausgelöst, bei welchem beide Depressoren (DR, DL) nahezu synchron aktiv sind. Schwache neuronale Erregung (IN) erscheint jeweils unmittelbar vor diesen synchronen Muskelaktivitäten, dauert dann während dieser noch etwas an, gefolgt von einer gehemmten Phase (Hemmung nicht vollständig) bis die Muskelaktivitäten beendet sind. Am Übergang erscheint dann noch einmal eine kurze Phase erhöhter neuronaler Aktivität B: Einfacher dorsal-taktiler Tarsusreiz (kontinuierliche Reizung bis zum Reizerfolg, gestrichelte Linie in der ST-Spur). Das Verhalten des Neurons ist im Vergleich zu A modifiziert: Es erscheint keine präaktive Phase neuronaler Erregung und das Neuron wird für die Dauer der synchronen Muskelaktivitäten vollständig gehemmt. Im Anschluss tritt eine Phase dauerhaft erhöhter neuronaler Aktivität über mehrere Sekunden auf. A-B: Je stärker die Muskelaktivität des ipsilateralen Mittelbeins (DR) ist, desto ausgeprägter ist die synchrone Hemmung der neuronalen Aktivität.

Abkürzungen: IN: Aktivität des Neurons; DR: Depressor trochanteris, rechtes Mittelbein; DL: Depressor tr., linkes Mittelbein; ST: dorsal-taktiler Tarsusreiz. 


\section{CIN-II(a)i1}

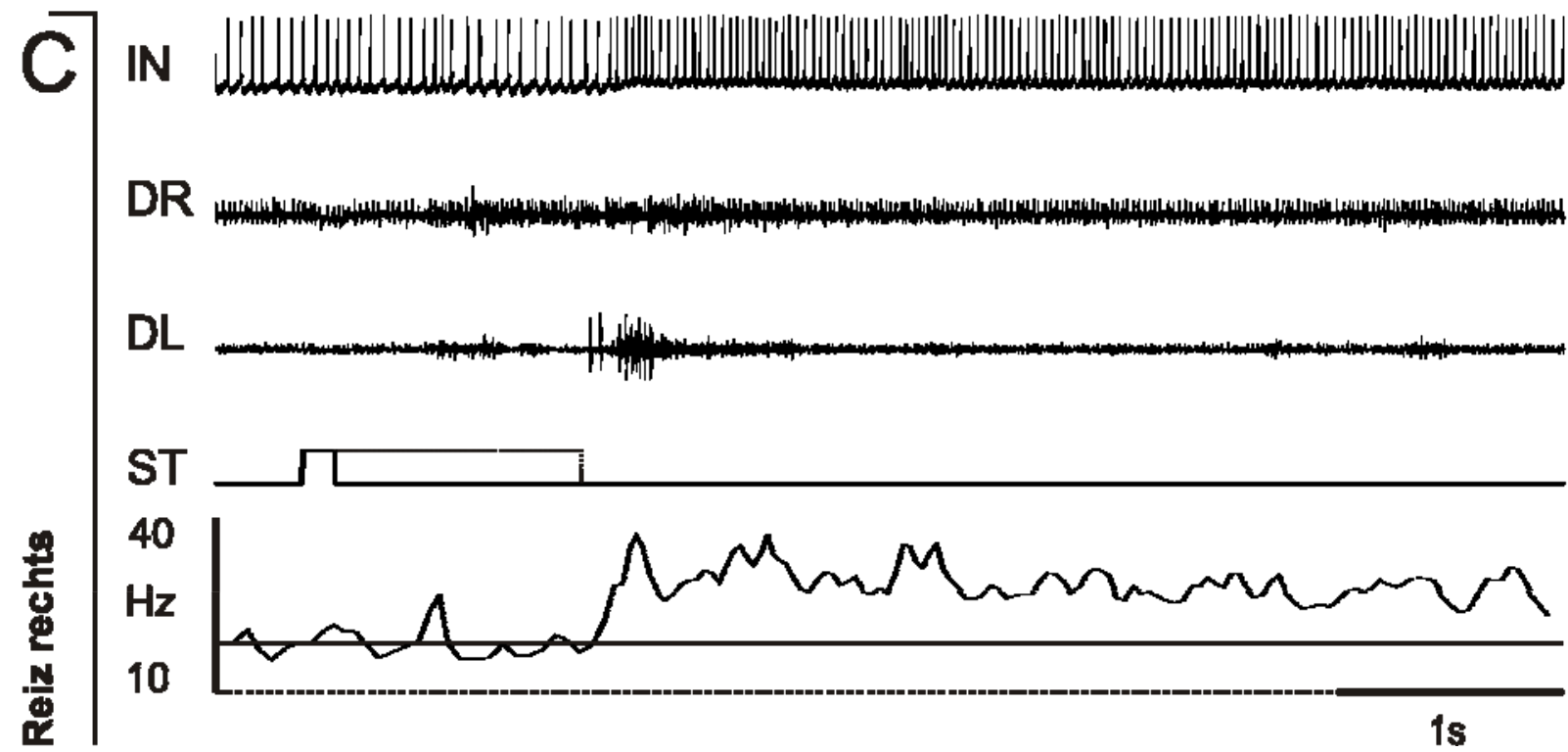

Abb. 32: C: Aktivität, Instantan- und Spontanfrequenzen des Neurons CIN-II(a)i1 und Muskelaktivitäten bei ipsilateraler Reizung durch einen einmaligen dorsal-taktilen Tarsusreiz am rechten Mittelbein (kontinuierliche Reizung bis zum Reizerfolg, Zeitraum der Reizung durch gestrichelte Linie in der ST-Spur angegeben). Dieser Ausschnitt zeigt eine latente dauerhafte Aktivität des kontralateralen Depressormuskels (DR) vor, während und auch nach der Reizung. Zweimal wird durch die kontinuierliche Reizung erkennbar ein Reflex ausgelöst, der sich durch Auftreten von synchron stärkerer Muskelaktivität (DR, DL; Auftreten von Spikes der jeweiligen fast-unit vom ipsi- oder kontralateralen Depressormuskel) äußert. Statt einer alternierenden Korrelation der neuronalen Aktivität mit der Muskelaktivität (wie in Abb. 31 A-B gezeigt) liegt hier eine synchrone vor. Bezüglich der ersten Reflexbewegung (erstmals Auftreten von synchron stärkerer Muskelaktivität in beiden Depressorableitungen) ist das Neuron bereits präaktiv für wenige Millisekunden. Beim zweiten Reflex erscheint erhöhte neuronale Erregung mehr oder weniger synchron mit der Muskelaktivität (beider Depressoren). An letztere synchron korrelierte Phase schließt sich eine tonische an, in welcher das Neuron über mehrere Sekunden lang bei einer dauerhaft erhöhten Frequenz aktiv ist. Es ist aber nicht auszuschließen, dass dies möglicherweise auch durch ein Ableitartefakt bedingt ist (Veränderung der intrazellulären Ableitbedingungen durch auftretende mechanische Kräfte bei Beinbewegungen).

Abkürzungen: IN: Aktivität des Neurons; DR: Depressor trochanteris, rechtes Mittelbein; DL: Depressor tr., linkes Mittelbein; ST: dorsal-taktiler Tarsusreiz.

neuronaler Erregung ein, deren Dauer etwa vergleichbar ist zur präaktiv erregten Phase (zwischen 200-300ms). Die prä- und postaktiven neuronalen Bursts zeigen aber nur schwache Erregung (bis maximal $30-40 \mathrm{~Hz}$ ), die das Niveau der Spontanfrequenz des Neurons in diesem Zeitraum nur geringfügig überschreiten. Eine weitere kontralaterale taktile Tarsusreizung (ebenfalls kontinuierlich) führt zu einer modifizierten neuronalen Reaktion (Abb. 31, B). Hier erscheint keine präaktive Phase neuronaler Erregung, stattdessen erstreckt sich die postaktiv erregte Phase über einen Zeitraum von mehreren Sekunden und bei einer Frequenz, die dauerhaft 
zwischen $30-40 \mathrm{~Hz}$ liegt. Zwar kann dies auch durch mechanisch bedingte Veränderungen der intrazellulären Ableitbedingungen begründet sein (Ableitartefakt, hervorgerufen durch die Muskelbewegungen), jedoch erscheint eine solche dauerhaft erhöhte neuronale Aktivität in vergleichbarer Weise auch einmal nach einer ipsilateralen taktilen Reizung am Tarsus des rechten Mittelbeins (Abb. 32, C). Die während dieser durch kontralaterale Reizung ausgelösten Reflexbewegung (Abb. 31, B) auftretenden Muskelaktivitäten der beiden Depressoren sind etwas stärker, was sich in vermehrtem Auftreten von Spikes der jeweiligen fast-unit synchron in beiden Muskelableitungen, vor allem bezüglich des ipsilateralen rechten Depressors zeigt. Auch während diesem ausgelösten Reflex wird das Neuron für exakt die Dauer der synchronen Muskelaktivität gehemmt. In diesem Fall ist die Hemmung vollständig, das Neuron ist in dieser Phase (150-200ms) inaktiv.

Nach ipsilateraler taktiler Reizung an der Dorsalseite des rechten Mittelbein-Tarsus (Abb. 32, C) erscheint eine neuronale Aktivität, die vergleichbar zu der in der Abbildung 31 B gezeigten ist, mit dem einzigen Unterschied, dass das Neuron nicht für die Dauer der synchron auftretenden stärkeren Muskelaktivität gehemmt wird. Das bedeutet, die neuronal erregte Phase beginnt bereits synchron mit dem Einsetzen stärkerer Muskelaktivität beider Depressoren, überdauert diese aber auch in diesem Fall um mehrere Sekunden, wobei die Burstfrequenz (Instantanfrequenz) dauerhaft zwischen $20-40 \mathrm{~Hz}$ liegt. Auch hier kann jedoch nicht ausgeschlossen werden, dass diese Phase von dauerhafter neuronaler Erregung auf ein Ableitartefakt zurückzuführen ist.

Hyper- und Depolarisation des Neurons haben das Membranpotential in der erwarteten Weise beeinflusst (nicht gezeigt). Darüber hinaus ließ sich jedoch kein indirekter Einfluss auch auf Muskelaktivitäten erkennen.

Kurzdiskussion:

Das Neuron CIN-II(a)i1 ähnelt von der morphologischen Struktur her einem Typ von Interneuronen, die in Schistocerca gregaria (Forskål) gefunden wurden und für welche der laterale „Looping“-förmige Verlauf des Axons als morphologisch kennzeichnend beschrieben worden ist (WATSON and BURROWS 1983; Fig.2.C). Neurone dieses Typs können sehr unterschiedliche Verzweigungsbereiche haben. Ultrastrukturelle Untersuchungen eines solchen Interneurons haben gezeigt, dass 
Verzweigungsbereiche mit Input- und Outputregionen nicht voneinander getrennt vorliegen. Input- und Outputsynapsen kommen entlang derselben Fasern mehr oder weniger gleich häufig vor und liegen oft nahe beieinander. Die Interneurone dieses Typs besitzen untereinander ähnliche physiologische Merkmale. Das von WATSON und BURROWS (1983) beschriebene Neuron wird depolarisiert und generiert Spikes bei taktilen Reizen an beiden Vorderflügeln, oder wenn einer dieser beiden aktiv bewegt (angehoben) wird. Wird der kontralaterale Vorderflügel der Heuschrecke in einer angehobenen Position gehalten, ist das Neuron tonisch bei erhöhter Spikefrequenz aktiv für die Dauer dieser Auslenkung. Auch durch taktile Reize an beiden Mittelbeinen, besonders aber an der Ventralseite der Tarsen, kommt es zur Ausbildung von Spikes. Letztere Form der Reizung (an der Ventralseite der Tarsen) und auch taktile Reizung an den Vorderflügeln oder deren Auslenkung wurde an diesem Präparat nicht getestet, aber das Interneuron CIN-II(a)i1 reagiert auch auf taktile Reizung an der jeweiligen Dorsalseite beider Mittelbeintarsen sowie auf taktile Reize an Haaren, die lateral zu beiden Seiten an der Vorderkante des Pronotums lokalisiert sind. Diese Haare sind in Gruppen lokalisiert und werden auch bei Bewegungen des Hinterkopfes ausgelenkt. Neben zahlreichen anderen proprio- und exterozeptiven Signalen ist ihr sensorisches Feedback wichtig bei der Flugsteuerung und Ausrichtung der Dorsalseite der Komplexaugen in Orientierung zum Licht (GOODMAN 1959). Da das Neuron CIN-II(a)i1 offensichtlich Input von sensorischen Neuronen aus diesem Bereich erhält, ist es theoretisch denkbar, dass es möglicherweise auch Teil eines Netzwerkes ist, das in den Kontext von Flug involviert ist. Das konnte aber in diesem Zusammenhang und bei der verwendeten Präparationsmethode nicht weiter überprüft werden.

Aber auch eine allgemeinere die Extremitäten koordinierende Funktion des Neurons CIN-II(a)i1 erscheint möglich. Das Neuron reagiert auf taktile Reizung sowohl an beiden Vorder- wie Mittelbeinen, könnte also auch an intersegmentaler Koordination der Extremitäten beteiligt sein, zumal es im ipsilateralen Konnektiv aufsteigt und somit auch Verzweigungen im Prothorakalganglion besitzt (Struktur dort nicht bekannt). Neuronale erhöhte Aktivität erscheint bei Reizung der Mittelbeine im Wesentlichen in mehr oder weniger streng alternierender Korrelation, in kurzen erregten Phasen unmittelbar vor und nach den synchron auftretenden Muskelaktivitäten, unterbrochen von einer dazwischen liegenden gehemmten Phase (Abb. 31, A-B). Diese Hemmung trat nur unidirektional bei kontralateraler Reizung 
auf, bei ipsilateraler Reizung beginnt die Phase neuronaler Erregung bereits wenige Millisekunden vor dem Einsetzen von Muskelaktivität beider Depressoren (synchrone Korrelation) und überdauert letztere dann allerdings in einer anschließenden tonischen Phase über mehrere Sekunden (Abb. 32, C). Die Hemmung scheint auch abhängig zu sein von der Stärke der ipsilateralen Muskelaktivität. In dem in Abbildung 31 A gezeigten Beispiel ist die Hemmung unvollständig, der ipsilaterale Depressor trochanteris (DR) zeigte zum Zeitpunkt des ersten Reflexes (erstmaliges Auftreten von synchron stärkerer Muskelaktivität) nur schwache Aktivität. Beim zweiten Reflex (Abb. 31, A) war seine Aktivität etwas stärker bei gleichzeitig stärkerer Hemmung des Neurons. Die stärkste Muskelaktivität des ipsilateralen Depressors erscheint nach einer weiteren kontralateralen Reizung (Abb. 31, B). Für die Dauer der synchron auftretenden Muskelaktivität wurde das Neuron hier vollständig gehemmt.

Eine direkte Verschaltung des Interneurons auf Motoneurone, oder gar eine direkt bilateral die Mittelbeine koordinierende Funktion ist aber schon allein aufgrund der morphologischen Struktur unwahrscheinlich. Das Neuron verzweigt mesothorakal eher median im Umfeld der dorsalen Kommissuren und intermediär als lateral und reicht in keinem Hemiganglion an die lateralen Neuropile (pLAC, aLAC) heran (Abb. 29-30). Die Verzweigungsbereiche liegen in beiden Hemiganglien in der näheren Umgebung der intermediären (DIT,VIT) und lateralen (LDT) Trakte sowie vom medianen (DMT) Trakt. Das Neuron könnte also hier auf andere Interneurone, möglicherweise sogar aus verschiedenen funktionalen Netzwerken (Flug, Laufen), verschalten und auch selbst Input erhalten. Varikositäten ließen sich an keiner der Verzweigungen eindeutig erkennen, könnten aber dennoch vorhanden sein, das lässt sich in der Färbung nicht erkennen. Generell erscheinen alle mesothorakalen Verzweigungen sowohl des Neuriten als auch der Kollaterale mehr oder weniger homogen. Das würde dem Befund aus der oben genannten ultrastrukturellen Beschreibung des anderen beschriebenen Interneurons vergleichbaren Typs (WATSON and BURROWS 1983) ganz gut entsprechen, nach welchem es keine getrennten Input- und Outputregionen gibt, sondern Output- und Inputsynapsen etwa gleichhäufig an allen Fasern vorkommen und zum Teil nahe beieinander liegen. Solche Daten liegen über das Interneuron CIN-II(a)i1 nicht vor. Jedoch ist dies eine bei kommissuralen Interneuronen vom Typ CIN-II häufiger zu machende Beobachtung, dass die Verzweigungen eines solchen Neurons im Mesothorakal- 
ganglion mehr oder weniger homogen erscheinen. Viele der genannten Interneurone wie auch dieses Neuron verzweigen gerade im Umfeld der dorsalen Kommissuren. Möglicherweise ist diese Region eine im jeweiligen Ganglion zentrale Schnittstelle, wo Integration von bilateraler und intersegmentaler Koordination der Extremitäten im jeweiligen Verhaltenskontext stattfindet und wo auch neuronale Netzwerke aus verschiedenen Funktionskreisen miteinander gekoppelt werden könnten.

Zusammenfassend lässt sich über das kommissurale Interneuron CIN-II(a)i1 sagen, dass es sowohl aufgrund seiner morphologischen Merkmale (mesothorakal homogen erscheinende Fasern im median gelegenen kommissuralen Umfeld), als auch wegen seiner eher unspezifischen Reaktion auf die verschiedensten Reizungen (taktile Reize unspezifisch an beiden Vorder- und Mittelbeinen und von lateral am Thorax befindlichen Haaren) zwar im Kontext der Koordination der Extremitäten involviert sein könnte, dass es aber wahrscheinlich eher eine zentralere integrierende Funktion hat und möglicherweise in mehrere verschiedene Funktionskreise (zum Beispiel Laufen, Flug) mit einbezogen sein kann. Neuronale für die Koordination der Extremitäten relevante Information könnte über solche kommissuralen median verzweigenden Interneurone bilateral zur Verfügung gestellt werden, da sie ja in beiden Hemiganglien verzweigen. Ihre Information könnte auf andere lokale oder intersegmentale Interneurone (möglicherweise auch solche vom Typ CIN-I, die bilateral verzweigen) übertragen werden, die ihrerseits auf die entsprechenden Motoneurone verschalten. 
3.2 Allgemeine morphologische und physiologische Merkmale von kommissuralen intersegmentalen Interneuronen

3.2.1 Kommissurale Interneurone vom Typ CIN-I mit bilateralem Verzweigungsmuster

Morphologie:

Dieser Neurontyp zeichnet sich durch lateral gelegene Verzweigungsbereiche in beiden mesothorakalen Hemiganglien aus (Abb. 33: Übersicht 1, kommissurale Interneurone Typ I). Zwar können einige solcher Neurone weiter nach lateral reichen als andere, aber in der Regel erstrecken sie sich in beiden Hemiganglien mindestens bis zu dem Bereich von einem oder beiden lateralen Neuropilregionen (aLAC, pLAC). Die Verzweigungen können extrem weit dorsal liegen, was häufiger der Fall ist, weniger häufig liegen sie im intermediären Bereich. Die extrem dorsal verzweigenden Ausläufer des Neuriten haben aber meistens noch einen oder wenige kleinere Nebenverzweigungen, die intermediär oder sogar bis nach ventral verzweigen. Oft liegen diese auf der Höhe relativ zu dem Bereich zwischen zwei sich longitudinal gegenüber liegenden Konnektiven, über die ganze Konnektivbreite und möglicherweise im Umfeld longitudinaler Trakte (zum Beispiel DIT, VLT, VIT und MVT). In vielen Fällen lassen sich bereits unter dem Fluoreszenzmikroskop an einzelnen Fasern Varikositäten erkennen. Die an diesen lokalen kleinen Aufschwellungen befindlichen Synapsen haben meistens - wenn dies auch nicht immer der Fall sein muss - Output-Charakter (BURROWS 1996, p. 58-60), daher können diese Strukturen auf einen möglichen lokalen Projektionsbereich des Neurons im Pro-, Meso-, oder Metathorakalganglion hinweisen. Dabei gibt es einerseits Verzweigungsbereiche, in welchen solche varikosen Fasern nebeneinander mit Fasern von eher dendritischem Charakter (nicht varikos, Vorkommen von meist Input-erhaltenden Synapsen) auftreten sowie andererseits solche, wo entweder überwiegend varikose oder hauptsächlich dendritische Fasern auftreten.

Bei Neuronen, in deren Verzweigungsbereichen in beiden Hemiganglien sowohl varikose wie dendritische Fasern nebeneinander vorkommen, gibt es häufiger auch 


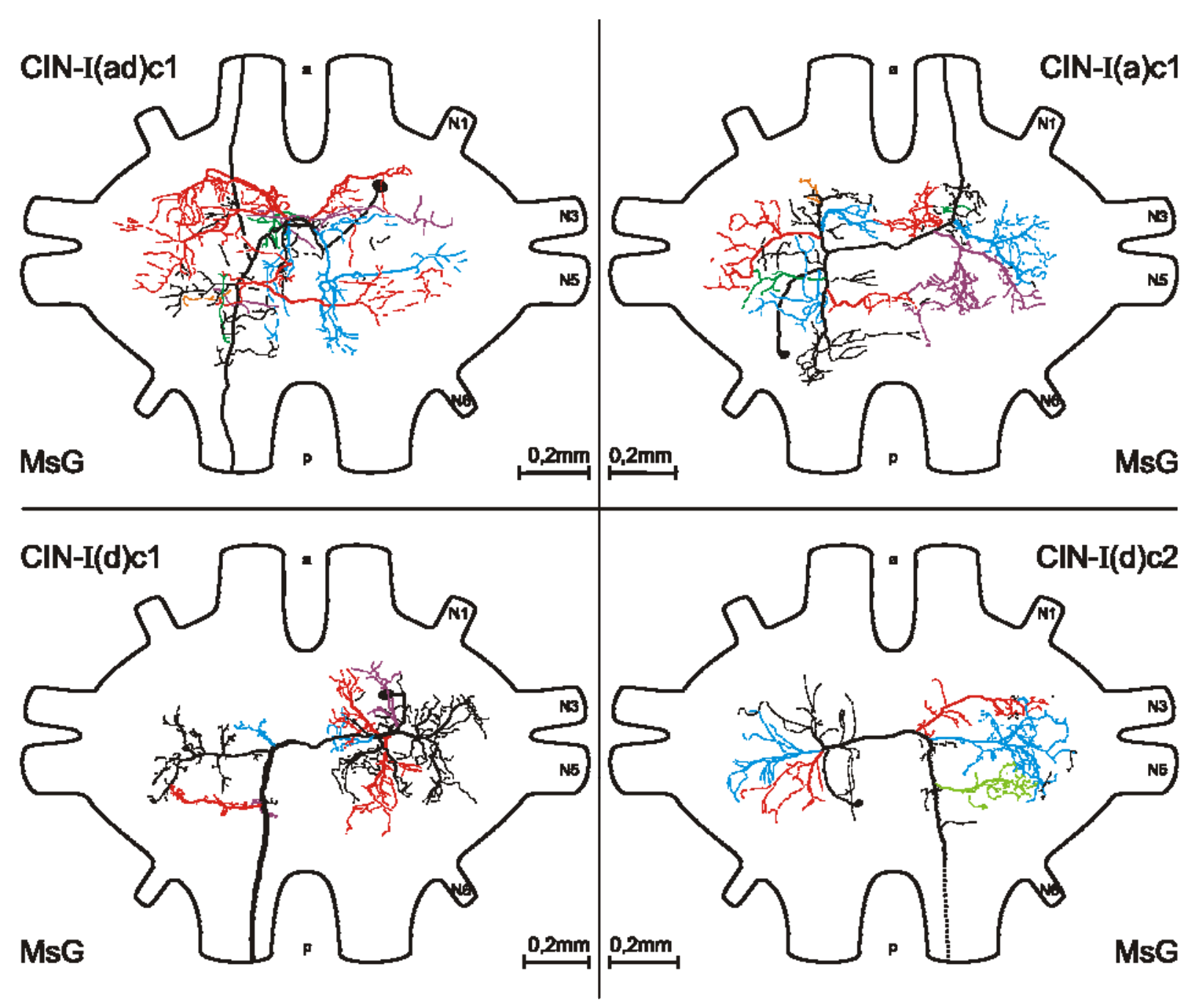

Abb. 33: Kommissurale Interneurone vom Typ CIN-I. Morphologische Übersicht und Vergleich von intersegmentalen kommissuralen Interneuronen mit bilateralem Hauptverzweigungsbereich (Übersicht 1). Alle hier dargestellten Neurone dieses Typs verlaufen vermutlich durch jeweils eine der dorsalen Kommissuren DCI (CIN-I(ad)c1), DCII (CIN-I(a)c1, CIN-I(d)c2), oder DCIII $(\mathrm{CIN}-\mathrm{I}(\mathrm{d}) \mathrm{c} 1)$. Sie haben ein relativ zur Somaposition im kontralateralen Konnektiv gelegenes Axon und bilaterale Verzweigungsbereiche, die meistens an die lateral gelegenen Neuropile (aLAC, pLAC) heranreichen und dorsal (CIN-I $(\mathrm{ad}) \mathrm{c} 1, \mathrm{CIN}-$ $\mathrm{I}(\mathrm{a}) \mathrm{c} 1)$ oder intermediär bis dorsal $(\mathrm{CIN}-\mathrm{I}(\mathrm{d}) \mathrm{c} 1, \mathrm{CIN}-\mathrm{I}(\mathrm{d}) \mathrm{c} 2)$ lokalisiert sind. Die Somata $(\varnothing: 15-30 \mu \mathrm{m})$ liegen ventral und posterior oder anterior auf der Höhe relativ zur gedachten Verbindungslinie durch zwei sich longitudinal gegenüber liegende Konnektive (Verbindungslinie entlang der inneren bzw. äußeren Randzone der Konnektive).

Abkürzungen: MsG: Mesothorakalganglion; a: anterior; p: posterior; N1-N6: Nerv 1-6; aLAC: anterior lateral association centre; pLAC: posterior lateral association centre; DCIDCIII: dorsal commissures I-III; Typographische Abkürzungen der Neurone: Siehe Liste der verwendeten Abkürzungen und Erläuterung im Abschnitt 2.6.1 (Teil: Versuchstiere, Material und Methoden). 
einzelne Verzweigungen oder Kollaterale, die separat vom primären Neuriten über eine andere dorsale Kommissur oder kommissuralen Trakt in das jeweils gegenüber liegende Hemiganglion ziehen und dort meist mit lokalen Verzweigungen überlappen (rekurrente Fasern). Solche strukturell vergleichbar aufgebauten Verzweigungsbereiche (varikose und dendritische Fasern in beiden Hemiganglien) liegen dann auch meist auf vergleichbarem Niveau dorsal oder intermediär bis dorsal (Neurone: CIN-I(ad)c1, Abb. 6; CIN-I(a)c1, Abb. 11). Dem gegenüber stehen solche Neurone, die kontralateral überwiegend varikose Kollaterale (mutmaßliche lokale Projektionsbereiche) und ipsilateral hauptsächlich eher dendritische (wahrscheinlich Inputerhaltende) Verzweigungen haben. Bei derartig strukturierten Verzweigungsbereichen kommt es seltener vor, dass einzelne Fasern über die Mittellinie in das jeweils gegenüber liegende Hemiganglion ziehen und damit zu geringerer bis keiner Überlappung zwischen den bilateralen Verzweigungsbereichen (Neurone: CIN$\mathrm{I}(\mathrm{d}) \mathrm{c} 1$, Abb. 14; CIN-I(d)c2, Abb. 18). Die ipsilateral vom Neuriten entspringenden Hauptverzweigungen mit eher dendritischem Charakter können dann auch in einer anderen Region intermediär oder manchmal sogar ventral verzweigen, im Gegensatz zu den kontralateralen und Varikositäten tragenden Kollateralen, die meist dorsal liegen (repräsentiert durch das Neuron: $\mathrm{CIN}-\mathrm{I}(\mathrm{d}) \mathrm{C} 2, \mathrm{Abb}$. 18). Bis auf eine Ausnahme haben die beschriebenen Interneurone vom Typ CIN-I einen primären Neuriten, dessen kommissuraler Teil die jeweilige dorsale Kommissur in der Regel nur einmal durchquert. Das Neuron CIN-I(ad)c1 (Abb. 6) passiert im Gegensatz dazu die Kommissur DCl zweimal.

Die gefundenen Neurone dieses Typs haben Somata in der Größe zwischen 15$30 \mu \mathrm{m}$. Diese liegen ventral entweder anterior oder posterior auf der Höhe einer gedachten longitudinalen Verbindungslinie durch zwei sich gegenüber liegende Konnektive (Verbindungslinie durch die innere oder äußere Randzone der Konnektive). Der primäre Neurit verläuft meist bogenförmig in Richtung der ZielKommissur. Ein mehr oder weniger breiter Teil des primären Neuriten ist auf Höhe dieser Kommissur verdickt und trägt alle Hauptverzweigungen. Diese liegen entweder ausschließlich ipsilateral (CIN-I(d)c1, Abb. 14; CIN-I(d)c2, Abb. 18), dann beginnt auf kontralateraler Seite der axonale Teil des Neurons, der große Kollaterale mit deutlich erkennbaren Varikositäten (lokale Projektionsbereiche) ausbilden kann, oder bilateral (CIN-I(ad)c1, Abb. 6; CIN-I(a)c1, Abb. 11), dann kommen dendritische 
Fasern in der Regel nebeneinander mit varikosen Fasern vor (Input- und Outputregionen liegen wahrscheinlich nebeneinander). Bei letzterem Typ kann das Axon dennoch mehrere und zum Teil auch große Kollaterale tragen, die oft rekurrent mit Verzweigungen des primären Neuriten überlappen. Die hier beschriebenen bilateral verzweigenden Interneurone haben ausschließlich kontralateral gelegene Axone. Diese können absteigend, aufsteigend oder durchlaufend sein. Keines der gefärbten Neurone dieses Typs verläuft in mehr als zwei axonalen Richtungen oder hat eine Crossover-Anordnung (ipsilateral auf- und kontralateral absteigend, oder umgekehrt).

Physiologie:

Alle gefundenen kommissuralen Interneurone mit bilateralen Verzweigungsbereichen vom Typ CIN-I sind in Ruhe tonisch (regelmäßiger Tonus oder rhythmisch) aktiv und zeigen während bilateral ablaufender Reflexe, die durch taktile Reizung an der Dorsalseite der Mittelbeintarsen ausgelöst werden, in der Regel erhöhte phasische Erregung (Bursts oder Spike-Salven), die strenger mit einer der beiden registrierten Muskelaktivitäten (M103a, Depressor trochanteris, ipsi- und kontralaterales Mittelbein) korreliert. Diese Korrelation kann eine alternierende (Abb. 13 B, 21 D, am Beispiel der Neurone CIN-I(a)c1 und CIN-I(d)c2 gezeigt) oder eine synchrone sein (zum Beispiel deutlich in Abb. $17 \mathrm{C}$ zu erkennen beim Neuron CIN-I(d)c1). Im Falle der alternierenden Korrelation erscheint der neuronale Burst zeitlich meist unmittelbar vor der nachfolgenden Muskelaktivität einer der beiden Depressoren (M103a), bei synchroner Korrelation beginnt die neuronal erhöhte Aktivität meist wenige Millisekunden früher (präaktive Phase) als die Aktivität einer der beiden Depressoren, dauert dann eine Weile synchron zu dieser an und kann vor Abklingen der Muskelaktivitäten beendet sein oder diese auch noch eine Weile überdauern. In der Regel erscheint neuronal erhöhte Aktivität nach einer Reizung fast ausnahmslos immer nur dann, wenn auch Muskelaktivitäten gemessen wurden und zwar im zeitlichen Kontext davon. Das betrifft auch intersegmental (und gegebenenfalls auch gleichzeitig bilateral) ablaufende Reflexe, die durch taktile Reizung entweder an der Dorsalseite einer der beiden Vorderbeintarsen oder von lateral am Thorax oder Abdomen befindlichen Haaren ausgelöst werden (nicht gezeigt). Neuronal erhöhte Aktivität erscheint auch hier fast immer nur im Zusammenhang mit auftretender 
Muskelaktivität. Selten hingegen tritt neuronale Erregung (sowohl bei bilateralen wie intersegmentalen Reflexen) etwa reaktiv im unmittelbaren Anschluss an die Reizung auf, wenn beide abgeleiteten Depressoren in diesem Kontext inaktiv sind. Manchmal werden Interneurone dieses Typs bei den getesteten bilateralen Reflexen unidirektional gehemmt. Dies scheint nur solche Neurone zu betreffen, deren Verzweigungen in beiden Hemiganglien eher von vergleichbarer Beschaffenheit sind (varikose Fasern kommen auf beiden Seiten nebeneinander mit nicht-varikosen vor) und die untereinander auch eine stärkere Tendenz zur gegenseitigen Überlappung haben. Dieser Fall ist hier repräsentiert durch das Neuron CIN-I(ad)c1 (Abb. 9, D und 10, E ). Bei allen anderen Neuronen, deren bilaterale Verzweigungsbereiche sowohl räumlich als auch aufgrund der Beschaffenheit ihrer Fasern (varikos oder eher dendritisch) voneinander abgegrenzt sind und weniger stark über die Mittellinie hinweg überlappen, kommt solch eine unidirektionale Hemmung nicht vor (Neuron CIN-I(d)c1, Abb. 16-17; Neuron CIN-I(d)c2, Abb. 20-21). Sie zeigen im Kontext der bilateralen Reflexe ein mehr oder weniger vergleichbares Verhalten, unabhängig vom gereizten Mittelbein und der Richtung des ablaufenden Reflexes. Ihre Spontanaktivität im Ruhezustand (vor jeglicher Reizung) zeigt hingegen oft einen fast rhythmischen Tonus, der sich durch meist kurze Spike-Salven auszeichnet, die durch kurze Verzögerungen mehr oder weniger stark voneinander abgesetzt sind (Neuron CIN-I(d)c1, Abb. 16-17).

Bei zwei Neuronen mit bilateralem Verzweigungsmuster (CIN-I(a)c1, CIN-I(d)c2) zeigt sich in den entsprechenden physiologischen Aufzeichnungen ein interessantes Verhalten. Im Kontext von aktiven Bewegungen der Mittelbeine (ohne Reizung) erscheint eine zur Muskelaktivität streng korrelierte neuronal erhöhte Aktivität mit folgender Besonderheit: In einer zeitlich klar von der umgebenden neuronalen Aktivität abgegrenzten separaten Phase erscheinen ausschließlich Spikes mit deutlich geringerer Amplitude (CIN-I(a)c1, Abb. 13; CIN-I(d)c2, Abb. 20-21). Diese Phase ist streng alternierend zur gemessenen Muskelaktivität korreliert und tritt immer am Übergang von erhöhter Aktivität mindestens einer der beiden Depressoren zu einer Phase auf, in welcher beide Depressoren inaktiv sind. Eine Interpretation der möglichen physikalisch-biologischen Hintergründe erfolgt in der jeweiligen Kurzdiskussion im Anschluss an die detaillierte Beschreibung des betreffenden Neurons. Beide Neurone habe solche bilateralen Verzweigungsbereiche, die räumlich in stärkerem Maße voneinander abgegrenzt sind und deren Fasern sich 
ipsi- und kontralateral auch aufgrund ihrer Beschaffenheit unterscheiden (überwiegend varikose Fasern in einem Hemiganglion gegenüber nebeneinander vorkommenden varikosen und dendritischen ( $\mathrm{CIN}-\mathrm{I}(\mathrm{a}) \mathrm{c} 1, \mathrm{Abb}$. 11) oder überwiegend dendritischen Fasern im gegenüber liegenden Hemiganglion ( $\mathrm{CIN}-\mathrm{I}(\mathrm{d}) \mathrm{c} 2, \mathrm{Abb}$. 18).

3.2.2 Kommissurale Interneurone vom Typ CIN-II mit medianem Verzweigungsmuster

Morphologie:

Kommissurale Interneurone dieses Typs haben einen median in beiden Hemiganglien gelegenen Hauptverzweigungsbereich, der sich in der Regel vom intermediären Bereich (zum Beispiel vom medialen ventralen Assoziationszentrum mVAC) bis nach dorsal (Übersichten 2-4. Abb. 34: CIN-II(ad)c1, CIN-II(ad)c3, CINII(ad)c4; Abb. 35: CIN-II(a)i1, CIN-II(a)i2; Abb. 36: CIN-II(d)c1, CIN-II(d)c2) oder von ventral bis dorsal erstreckt (Abb. 34: CIN-II(ad)c2; Abb. 35: CIN-II(ad)c5, CINII(ad)c6; Abb. 36: CIN-II(a)c1, CIN-II(a)c2). Sie verzweigen oft zu beiden Seiten nahe der Mittellinie (Umgebung der dorsalen Kommissuren) und diese Verzweigungen setzen sich meist auf dorsalem Niveau in die Peripherie fort. Aufgrund einer räumlichen Trennung durch die kommissuralen Regionen im Ganglion entsteht nicht selten der Eindruck einer bilateral organisierten Verzweigungsstruktur (Abb. 34: CIN-II(ad)c1; Abb. 35: CIN-II(a)i1; Abb. 36: CINII(d)c2). Im Gegensatz zu den Typ CIN-I Neuronen bleibt der gesamte Verzweigungsbereich der Neurone vom Typ CIN-II aber auf die mediane Region beschränkt und die Verzweigungen reichen selten über die laterale Ausdehnung der ipsi- oder kontralateralen Konnektive hinweg (gedachte longitudinale Verbindungslinie durch zwei sich longitudinal gegenüber liegende Konnektive, entlang ihrer jeweiligen lateralen Randzone). Einige Neurone haben auch noch einen lateralen Nebenverzweigungsbereich durch eine größere unilaterale Kollaterale, die dann lateral und intermediär bis dorsal bis an eines der beiden lateralen Neuropile (pLAC, aLAC) heranreicht (Abb. 34: CIN-II(ad)c1; Abb. 35: CIN-II(ad)c6; Abb. 36: CIN$\mathrm{II}(\mathrm{d}) \mathrm{c} 1, \mathrm{CIN}-\mathrm{II}(\mathrm{d}) \mathrm{c} 2)$.

Der primäre Neurit verläuft ebenso wie jener der bilateral verzweigenden Inter- 
neurone durch jeweils eine der dorsalen Kommissuren DCI-DCVI, aber bei keinem der gefundenen Neurone durchquert er diese mehrfach. Vielmehr liegen der primäre Neurit selbst oder manche seiner Verzweigungen in größerer Dichte oft in nächster Nähe der Mittellinie und der dorsalen Kommissuren (sowohl bezüglich der horizontalen wie der vertikalen Ebene). Dabei gibt es hauptsächlich (aber nicht ausschließlich) unilateral oder bilateral in der Umgebung der Kommissuren verzweigende Neuriten. Verläuft der primäre Neurit selbst in nächster Nähe, bildet er manchmal eine ringförmig die dorsalen Kommissuren umgebende Struktur aus (Abb. 34: CIN-II(ad)c2; Abb. 36: CIN-II(d)c2). Ausgehend von solch einem ringförmigen Teil des primären Neuriten können sich seine weiteren Verzweigungen weiter nach dorsal und ventral in den Bereich der ventralen Kommissuren fortsetzen (repräsentiert durch das Neuron CIN-II(ad)c2 in Abb. 34).

Die Somata der gefundenen Neurone liegen in der Größenordnung zwischen 15-40 $\mu \mathrm{m}$. Sie liegen ventral und meistens posterior oder anterior im Bereich zwischen den beiden Konnektiven (näher zur Mittellinie hin), manchmal auch in der Region, die in der gedachten Verlängerung der beiden sich gegenüber liegenden Konnektive liegt.

Sowohl ipsi- als auch kontralateral gelegene Verzweigungen des Neuriten erstrecken sich selten über die laterale Ausdehnung zweier sich longitudinal gegenüber liegender Konnektive hinaus (repräsentiert durch das Neuron CIN-II(a)i1 in Abb. 35). Der Verlauf des primären Neuriten zur Ziel-Kommissur hin ist oftmals nicht so ausgeprägt bogenförmig wie bei den Typ CIN-I Neuronen, manchmal kann die Strecke bis zum kommissuralen Prozess relativ kurz sein. Im Umfeld des kommissuralen Abschnitts ist der primäre Neurit mehr oder weniger stark verdickt und trägt alle Hauptverzweigungen, die aber immer sowohl im ipsi- wie auch kontralateralen Hemiganglion verzweigen. Eine klare Trennung wie bei manchen Neuronen vom Typ CIN-I in ipsilaterale Hauptverzweigungen (Neurit) und kontralateral gelegene Kollaterale (ausgehend vom Axon) kommt bei keinem der gefundenen Neurone vom Typ CIN-II vor. Es ist möglich, dass an vielen ihrer Fasern auch Varikositäten vorhanden sind. Bei den Neuronen CIN-II(ad)c2, CIN-II(ad)c5 und CIN-II(a)c1 (Abb. 34-36) lassen sich solche varikosen Fasern eindeutig erkennen. Hier liegen sie in deutlich abgetrennten Verzweigungsbereichen vor, dendritische und varikose Fasern scheinen also bei diesen genannten Neuronen 


\section{Kommissurale Interneurone Typ II}

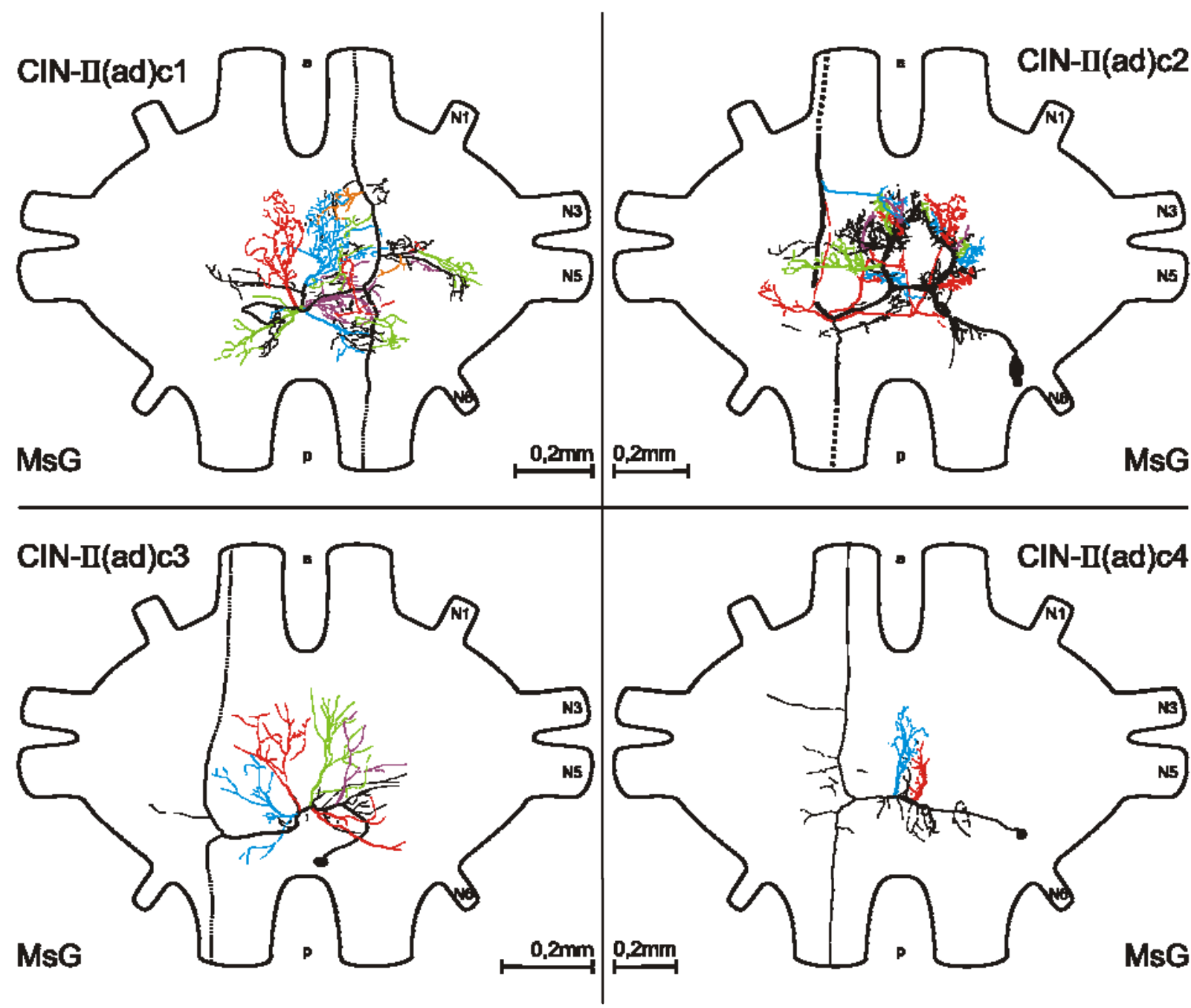

Abb. 34: Kommissurale Interneurone vom Typ CIN-II. Morphologische Übersicht und Vergleich von intersegmentalen kommissuralen Interneuronen mit medianem Hauptverzweigungsbereich (Übersicht 2).

Alle hier dargestellten Neurone dieses Typs verlaufen vermutlich durch jeweils eine der dorsalen Kommissuren DCIV (CIN-II(ad)c2), DCV (CIN-II(ad)c1, CIN-II(ad)c4) oder DCVI $(\mathrm{CIN}-\mathrm{II}(\mathrm{ad}) \mathrm{c} 3)$. Sie haben ein relativ zur Somaposition im kontralateralen Konnektiv gelegenes Axon und einen medianen Hauptverzweigungsbereich, der hauptsächlich intermediär bis dorsal lokalisiert ist (beim Neuron CIN-II(ad)c2: ventral bis dorsal). Die Verzweigungen reichen mit Ausnahme von unilateral gelegenen Kollateralen ( $\mathrm{CIN}-\mathrm{II}(\mathrm{ad}) \mathrm{c} 1)$ in keinem Hemiganglion deutlich über die laterale Ausdehnung jeweils der beiden sich longitudinal gegenüber liegenden Konnektive hinaus. Die Somata $(\varnothing: 15-40 \mu m)$ liegen ventral und posterior, entweder relativ zum Bereich, der in Verlängerung zweier sich longitudinal gegenüber liegender Konnektive liegt (Verbindungslinie entweder durch die innere oder äußere Randzone der Konnektive) oder im Bereich nahe der Mittellinie.

Abkürzungen: MsG: Mesothorakalganglion; a: anterior; p: posterior; N1-N6: Nerv 1-6; DCIV-DCVI: dorsal commissures IV-VI; Typographische Abkürzungen der Neurone: Siehe Liste der verwendeten Abkürzungen und Erläuterung im Abschnitt 2.6.1 (Teil: Versuchstiere, Material und Methoden). 


\section{Kommissurale Interneurone Typ II}

Übersicht 3

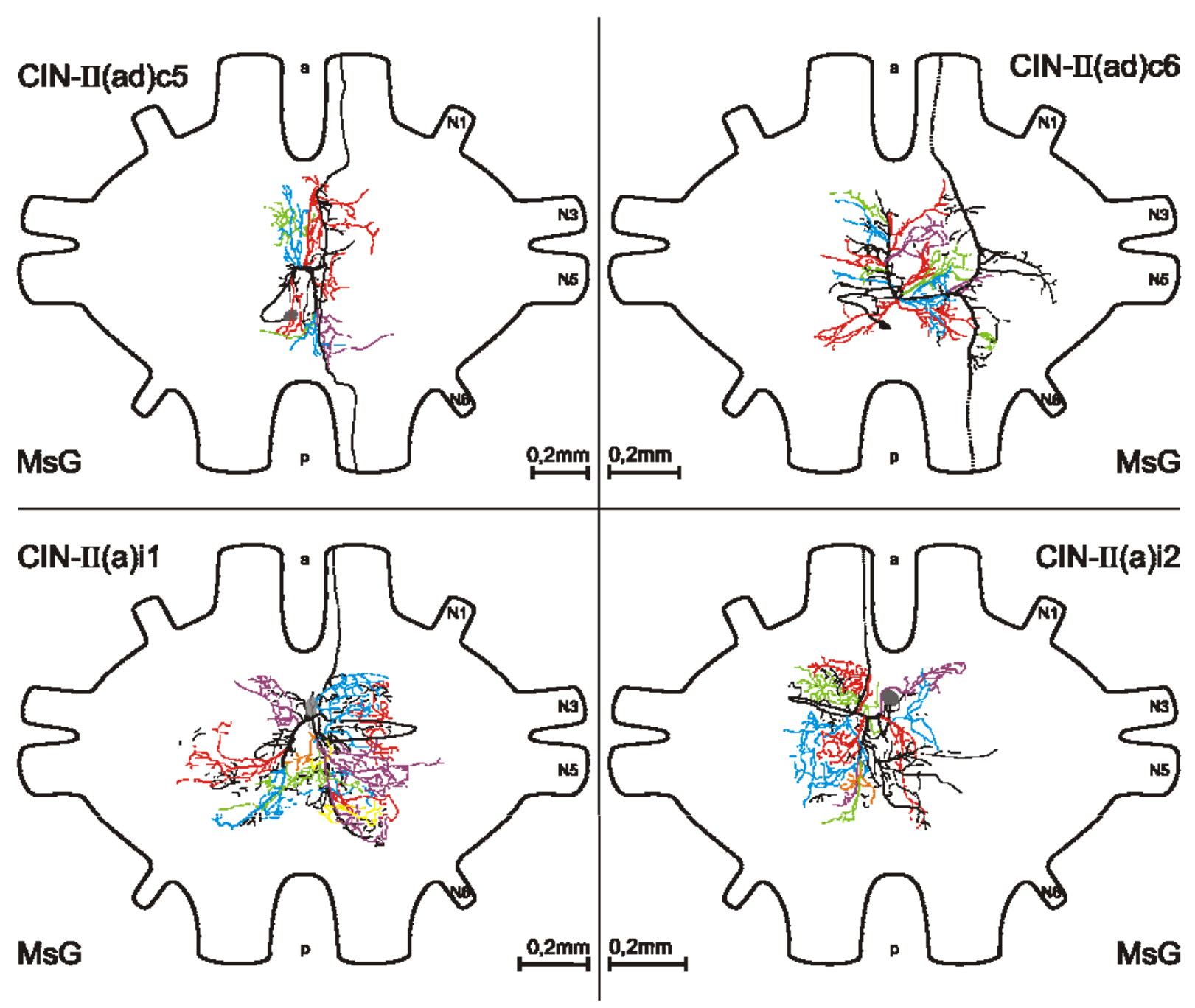

Abb. 35: Kommissurale Interneurone vom Typ CIN-II. Morphologische Übersicht und Vergleich von intersegmentalen kommissuralen Interneuronen mit medianem Hauptverzweigungsbereich (Übersicht 3).

Alle hier dargestellten Neurone dieses Typs verlaufen vermutlich durch jeweils eine der dorsalen Kommissuren DCI-DCII (CIN-II(a)i1, CIN-II(a)i2), DCIII (CIN-II(ad)c5) und DCIV (CIN-II(ad)c6). Sie haben einen medianen Hauptverzweigungsbereich, der intermediär bis dorsal (CIN-II(a)i1, CIN-II(a)i2) oder ventral bin dorsal (CIN-II(ad)c5, CIN-II(ad)c6) lokalisiert ist. Die Verzweigungen reichen mit Ausnahme von unilateral gelegenen Kollateralen ( $\mathrm{CIN}-\mathrm{II}(\mathrm{ad}) \mathrm{c} 6)$ in keinem Hemiganglion wesentlich über die laterale Ausdehnung zweier sich longitudinal gegenüber liegender Konnektive hinaus. Ihre Axone verlaufen relativ zur Somaposition im ipsi- oder kontralateralen Konnektiv. Die Somata $(\varnothing: 15-40 \mu \mathrm{m})$ liegen ventral, anterior oder posterior im Bereich nahe der Mittellinie (zwischen den Konnektiven).

Abkürzungen: MsG: Mesothorakalganglion; a: anterior; p: posterior; N1-N6: Nerv 1-6; DClDCIV: dorsal commissures I-IV; Typographische Abkürzungen der Neurone: Siehe Liste der verwendeten Abkürzungen und Erläuterung im Abschnitt 2.6.1 (Teil: Versuchstiere, Material und Methoden). 


\section{Kommissurale Interneurone Typ II}

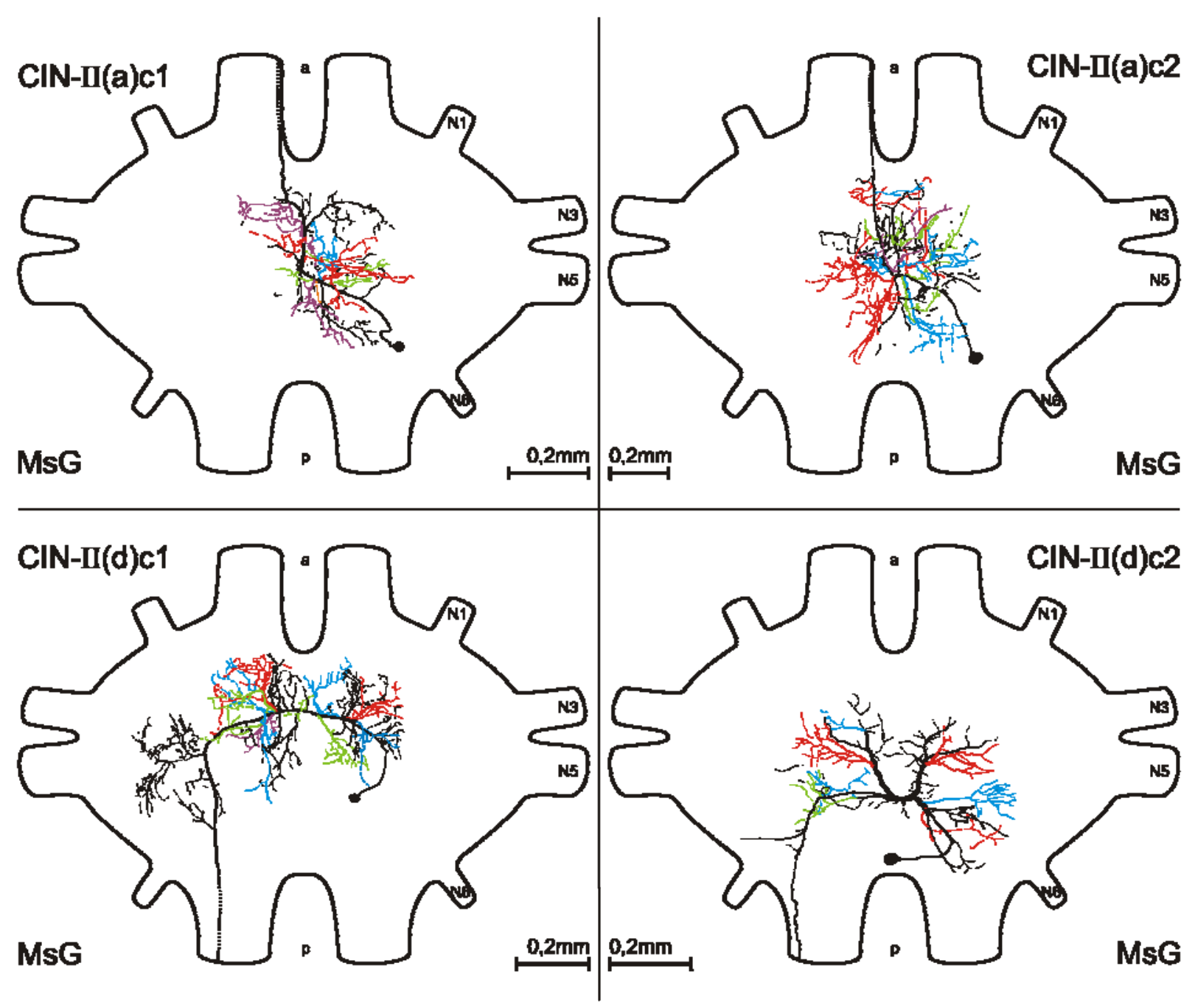

Abb. 36: Kommissurale Interneurone vom Typ CIN-II. Morphologische Übersicht und Vergleich von intersegmentalen kommissuralen Interneuronen mit medianem Hauptverzweigungsbereich (Übersicht 4).

Alle hier dargestellten Neurone dieses Typs verlaufen vermutlich durch jeweils eine der folgenden Kommissuren: DCI (CIN-II(d)c1), DCIII (CIN-II(a)c2), DCIV (CIN-II(d)c2) und SMC (CIN-II(a)c1). Sie haben ein relativ zur Somaposition im kontralateralen Konnektiv gelegenes Axon und einen medianen Hauptverzweigungsbereich, der intermediär bis dorsal (CIN-II(d)c1, CIN-II(d)c2) oder ventral bis dorsal (CIN-II(a)c1, CIN-II(a)c2) lokalisiert ist. Ihre jeweiligen Verzweigungen reichen mit Ausnahme von unilateral gelegenen Kollateralen (CIN-II(d)c1, CIN-II(d)c2) in keinem Hemiganglion deutlich über die laterale Ausdehnung jeweils zweier sich longitudinal gegenüber liegender Konnektive hinaus. Die Somata $(\varnothing: 15-30 \mu \mathrm{m})$ liegen ventral, entweder meist posterior relativ zum Bereich, der in Verlängerung zweier sich longitudinal gegenüber liegender Konnektive liegt (Verbindungslinie entlang der inneren oder äußeren Randzone dieser Konnektive), oder beim Neuron CIN-II(d)c2 posterior nahe der Mittellinie.

Abkürzungen: MsG: Mesothorakalganglion; a: anterior; p: posterior; N1-N6: Nerv 1-6; aLAC: anterior lateral association centre; pLAC: posterior lateral association centre; $\mathrm{DCl}-$ DCIV: dorsal commissures I-IV; SMC: supramedian commissure; Typographische Abkürzungen der Neurone: Siehe Liste der verwendeten Abkürzungen und Erläuterung im Abschnitt 2.6.1 (Teil: Versuchstiere, Material und Methoden). 
nicht nebeneinander vorzukommen. Bei den meisten anderen hier gezeigten Neuronen vom Typ CIN-II ist das Vorhandensein von Varikositäten nicht eindeutig erkennbar. Ihre Fasern wirken insgesamt feiner, verglichen mit betreffenden Neuronen vom Typ CIN-I, die deutlich erkennbare Varikositäten tragen. Falls sie jedoch Varikositäten tragen, liegen solche Fasern in beiden Hemiganglien meistens nebeneinander mit eher dendritischen Fasern vor. Unter den hier gezeigten könnte das auf die Neurone CIN-II(ad)c1, CIN-II(ad)c6, CIN-II(a)i1, CIN-II(a)i2 und CINII(a)c2 (Abb. 34-36) zutreffen. Input- und Outputregionen könnten also bei diesem Neurontyp häufiger nebeneinander vorkommen.

Einige Neurone besitzen unilateral mehr oder weniger große Nebenkollaterale und damit wahrscheinlich einen unilateralen klar abgetrennten Neben-Projektionsbereich (Abb. 34-36: betrifft die Neurone: CIN-II(ad)c1, CIN-II(ad)c2, CIN-II(ad)c3, CIN$\mathrm{II}(\mathrm{ad}) \mathrm{c} 4, \mathrm{CIN}-\mathrm{II}(\mathrm{ad}) \mathrm{c} 6, \mathrm{CIN}-\mathrm{II}(\mathrm{d}) \mathrm{c} 1, \mathrm{CIN}-\mathrm{II}(\mathrm{d}) \mathrm{c} 2)$. An den Fasern der Kollaterale lassen sich Varikositäten meist auch deutlich erkennen. Dennoch können aber wie oben beschrieben trotz solcher unilateralen Neben-Projektionsbereiche im Mesothorakalganglion auch Teile der medianen Hauptverzweigungen von varikosem Charakter sein. Eine räumliche Trennung innerhalb des medianen Verzweigungsbereiches in eine ipsilateral gelegene Input- und eine kontralateral gelegene Outputregion (oder umgekehrt) kommt bei keinem dieser Neurone vor. Die Neurone CIN-II(ad)c5 (Abb. 35) und CIN-II(a)i1 (Abb. 36) besitzen aber möglicherweise eine median-ventral gelegene Inputregion (eher dendritische Fasern) und einen mediandorsalen lokalen Projektionsbereich. Die Fasern ihrer median bis dorsal gelegenen Verzweigungen scheinen zumindest von varikosem Charakter zu sein.

Genauso wie Input- und Outputregionen bei diesem Neurontyp tendenziell häufiger nebeneinander vorkommen, so ist auch der Grad der Überlappung von ipsilateralen mit kontralateralen Verzweigungen tendenziell stärker. Viele einzelne Ausläufer können kommissural sein und ziehen über die Mittelinie hinweg in das jeweils gegenüber liegende Hemiganglion. Die Axone der Typ CIN-II Neurone verlaufen am häufigsten im kontralateral gelegenen Konnektiv. Zwei Neurone (Abb. 35; CIN-II(a)i1, CIN-II(a)i2) haben ein im ipsilateralen Konnektiv aufsteigendes Axon, das im Mesothorakalganglion einen „Looping“-förmigen Verlauf hat. Sie sind von der morphologischen Gestalt her vergleichbar mit einem Interneuron, für welches dieser 
„Looping“-förmige Verlauf des Axons als kennzeichnend beschrieben ist (WATSON and BURROWS 1983). Liegt das Axon im kontralateralen Konnektiv, so kann es dort aufsteigend, absteigend oder durchlaufend sein. Wie bei den bilateral verzweigenden kommissuralen Interneuronen (Typ CIN-I) hat keines der gefundenen Neurone periphere Verzweigungen (in anderen Ganglien) in mehr als zwei axonalen Richtungen oder eine „Crossover“-Anordnung vom Axon (ipsilateral aufsteigend und kontralateral absteigend oder umgekehrt).

Physiologie:

Alle gefundenen kommissuralen Interneurone mit medianem Hauptverzweigungsbereich vom Typ CIN-II sind in Ruhe tonisch aktiv. Neben Neuronen, deren Spontanaktivität sich durch einen eher regelmäßigen Tonus auszeichnet, gibt es auch unter innen manchmal solche, die dabei eher rhythmisch aktiv sind. Das betrifft die Neurone CIN-II(ad)c2 und CIN-II(a)i2 (physiologische Daten nicht gezeigt). Genau wie bei den Typ CIN-I Interneuronen erscheinen dann typischerweise kurze Bursts, die durch kürzere Phasen der Inaktivität mehr oder weniger deutlich voneinander abgesetzt sind. Alle Neurone zeigen eine erhöhte neuronale Aktivität oder eine gehemmte Phase im Kontext von durch Reizung ausgelösten Reflexen. Das betrifft sowohl bilaterale Reflexe, wenn dorsale taktile Tarsusreize an den Mittelbeinen gegeben werden, als auch intersegmentale Reflexe, wenn entsprechend die Vorderbeintarsen oder lateral am Thorax und/oder Abdomen befindliche Haare taktil gereizt werden. Die Korrelation zur zeitgleich mitregistrierten Muskelaktivität kann dabei manchmal etwas variabler sein als bei den bilateral verzweigenden Interneuronen vom Typ CIN-I. In vielen Fällen zeigt sich eine synchron (für eine Weile oder über die gesamte Dauer) zur Muskelaktivität einer der beiden abgeleiteten Depressoren korrelierte neuronale Erregung mit meist kurzer präaktiver Phase des Neurons (CIN-II(d)c1 in Abb. 27-28, CIN-II(a)i1 in Ab. 31-32). Die Korrelation ist oft nicht streng und die vor Einsetzen der Muskelaktivität präaktive Phase der neuronalen Erregung kann mal etwas länger und mal etwas kürzer dauern oder im Extremfall gibt es keine präaktive Phase und die neuronal erhöhte Aktivität erscheint genau synchron mit der Muskelaktivität (gut zu erkennen in Abb. 31, am Beispiel des Neurons CIN-II(a)i1). Eine bei bilateralen Reflexen unidirektional auftretende Hemmung (entweder bei ipsi- oder bei kontralateraler Reizung) ist bei 
diesen Neuronen häufig zu beobachten (Abb. 24 und 31, am Beispiel der Neurone CIN-II(ad)c1 und CIN-II(a)i1 gezeigt). Sie betrifft oft (aber nicht ausschließlich) Interneurone, die ein im kontralateralen Axon durchlaufendes Axon und einen primären Neuriten mit ringförmigem Aufbau in der näheren Umgebung der dorsalen Kommissuren haben. Solch eine Hemmung des betreffenden Neurons tritt meist genau synchron korreliert zur Aktivität einer der beiden abgeleiteten Depressoren auf. Eine gegebenenfalls zu diesem Zeitpunkt vor der Muskelaktivität erscheinende präaktive Phase von neuronaler Erregung bleibt meist erhalten. Im Anschluss an die gehemmte Phase kann eine weitere erregte Phase folgen, die oft erst nach längerer Zeit langsam abklingt (Abb. 24, CIN-II(ad)c1; Abb. 31-32, CIN-II(a)i1). Die zeitliche Korrelation der prä- und postaktiven Phase (neuronale Erregung) ist bei solchen Reflexen, während derer das Neuron für eine bestimmte Phase auch gehemmt wird, vergleichbar wie bei entsprechend in der entgegen gesetzten Richtung ablaufenden Reflexen ohne Hemmung des Neurons. Durch die phasische Hemmung des Neurons ergibt sich jedoch eine zur Muskelaktivität alternierend korrelierte Neuronaktivität im Gegensatz zur ansonsten synchronen Korrelation. Auch die beiden Neurone CINII(a)i1 und CIN-II(a)i2 (Abb. 35), deren jeweiliges Axon einen „Looping“-förmigem Verlauf aufweist und die morphologisch eine Ähnlichkeit zu dem von WATSON und BURROWS (1983) beschriebenen Interneuron-Typ haben, werden während bilateraler Reflexe unidirektional (CIN-II(a)i1; Abb. 31-32) bzw. bidirektional (CINII(a)i2; physiologische Daten nicht gezeigt) streng synchron zu mindestens einer der beiden gemessenen Muskelaktivitäten gehemmt.

Neben einer möglichen die Extremitäten bilateral und gegebenenfalls auch gleichzeitig intersegmental koordinierenden Funktion könnten die großen Interneurone vom Typ CIN-II auch noch zu anderen funktionalen Netzwerken gehören. Neben der nicht immer streng zur Muskelaktivität korrelierten neuronalen Erregung im Kontext der durch Reizung ausgelösten Reflexe erscheint erhöhte neuronale Aktivität auch in einigen Fällen unmittelbar im Anschluss an die Reizung selbst und ohne dass in diesem Kontext auch Muskelaktivität auftritt (das betrifft die Neurone CIN-II(ad)c2 und $\mathrm{CIN}-\mathrm{II}(\mathrm{a}) \mathrm{i} 2$, physiologische Daten nicht gezeigt). Allerdings ist in diesem Zusammenhang anzumerken, dass sich an manchen Präparaten nur noch schwach sichtbare Reflexe auslösen lassen. In manchen Fällen erscheinen zusätzlich zu der zur Muskelaktivität korrelierten neuronal erhöhten Aktivität jedoch auch noch Bursts 
unabhängig von Reizung und Auftreten von Muskelaktivität.

3.3 Licht- und elektronenmikroskopische Aufnahmen von medianen Sagittalschnitten des Mesothorakalganglions

Um die in den Vorbemerkungen genannten Fragestellungen nach der äußeren Begrenzung der dorsalen Kommissur DCl, nach der Anzahl der sie passierenden Zellen und deren mittlerem Durchmesser zu beantworten, wurden mediane Sagittalschnitte (Semi- und Ultradünnschnitte) des Mesothorakalganglions im Bereich nahe der Mittelinie angefertigt und für die Licht- und Elektronenmikroskopie vorbereitet. Die Ergebnisse sind in der Abbildung 37 gezeigt. Eine generelle Erkenntnis vom Studium dieser Schnittserien ist, dass sich die dorsalen Kommissuren in vielen Schnitten - selbst in solchen, die median nahe der Mittellinie gelegen sind - gar nicht eindeutig erkennen lassen, beziehungsweise, dass sich ihre äußere Begrenzung nicht genau bestimmen lässt und nicht klar ist, welche der meist nicht ganz genau quer angeschnittenen - Fasern zur Kommissur gehören und welche nicht (Abb. 37, A-B und D). Die Profile der durch die Kommissuren verlaufenden Axone beziehungsweise der primären Neuriten scheinen oft nicht kompakt nebeneinander zu liegen (sind in der Kommissur offensichtlich nicht immer kompakt gebündelt). Dieses Problem betrifft auch solche Schnitte, die nur wenig von der Mittellinie entfernt sind und gilt dann für alle dorsalen Kommissuren im Allgemeinen. In besonderem Maße scheint dies jedoch im Falle der dorsalen Kommissur DCl zuzutreffen. Die Abbildung 37 (A-B) zeigt einen solchen Sagittalschnitt, der median im Bereich zwischen den Konnektiven vom Ganglion abgetrennt wurde. Er ist zwar nicht exakt median lokalisiert, aber dennoch nicht weit von der Mittellinie entfernt. Dass hier in Längsrichtung angeschnittene Profile von Fasern longitudinaler Trakte erscheinen liegt daran, dass manche dieser Trakte (wie beispielsweise der dorsal median tract, DMT und der ventral median tract, VMT; nach PFLÜGER et al. 1988; Abb. 4) nahe der Mittellinie verlaufen. Wie in der Abbildung 37 (A-B) zu erkennen ist, liegen die Profile von angeschnittenen Axonen und primären Neuriten in der dorsalen Kommissur DCl weniger kompakt gebündelt vor, verglichen mit solchen der benachbarten Kommissur DCIII. Viele Fasern der Kommissur DCl sind nicht genau quer, sondern eher schräg angeschnitten, so dass 

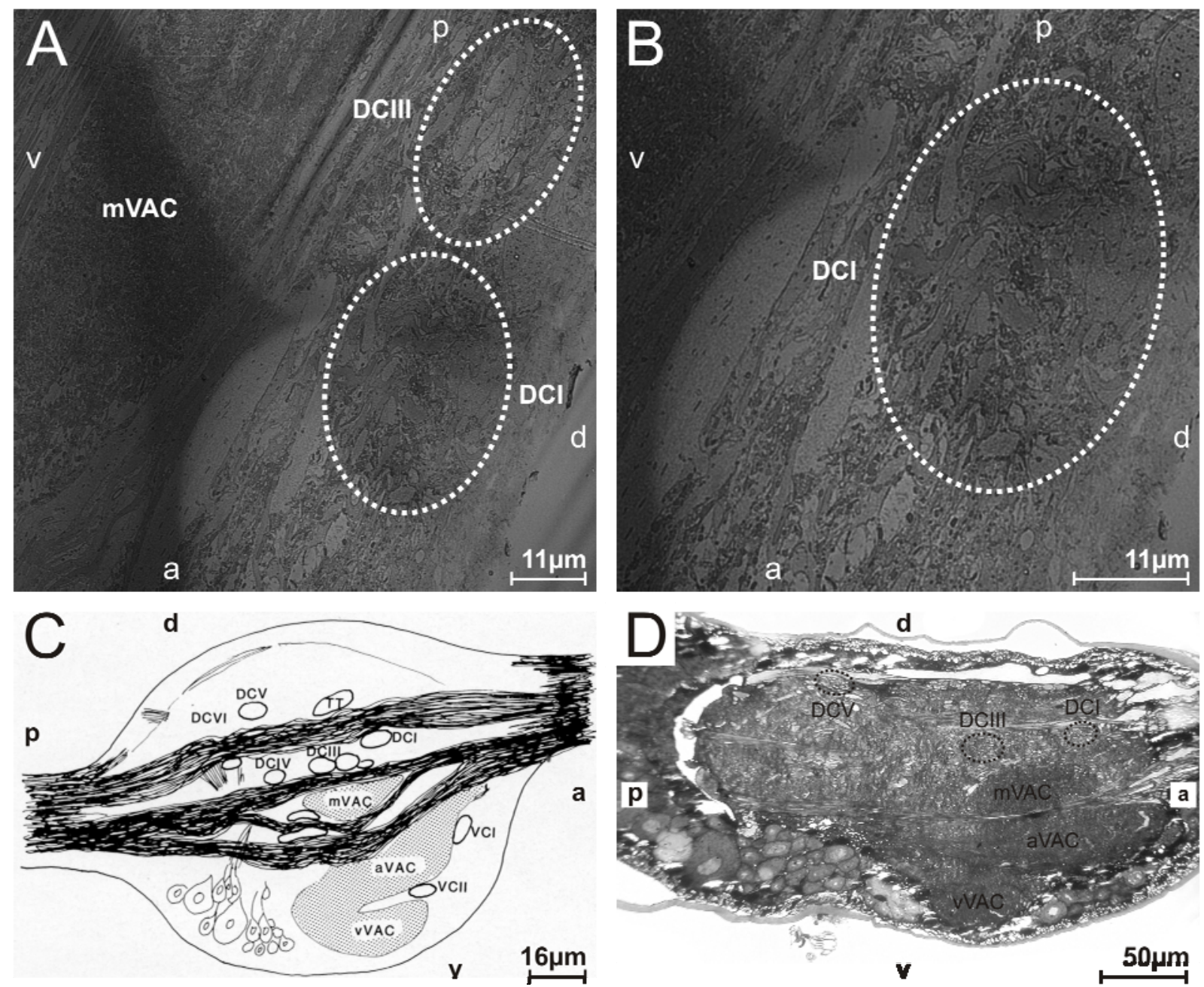

Abb. 37: Dorsale Kommissuren im Mesothorakalganglion der Wanderheuschrecke. Mediane Sagittalschnitte bei licht- (D) und elektronenmikroskopischer Vergrößerung (A, B).

A: In diesem Ausschnitt sind die dorsalen Kommissuren DCI und DCIII gezeigt. Die Profile von quergeschnittenen Axonen bzw. von Neuriten der Kommissur DCIII liegen im Vergleich zu solchen der Kommissur DCl kompakter nebeneinander und haben einen größeren mittleren Durchmesser. Kommissuren und benachbarte Strukturen wie zum Beispiel angeschnittene Profile von Fasern der longitudinalen Trakte und ventrale Neuropile sind auch in Ausschnitt C gezeigt.

B: Vergrößerter Ausschnitt aus A, der die dorsale Kommissur DCl zeigt. Manche der angeschnittenen Profile lassen sich nicht eindeutig dieser Kommissur zuordnen. Insgesamt liegen sie etwas verstreuter im Vergleich zu solchen der Kommissur DCIII (Ausschnitt A) und oftmals sind sie nicht genau im Querschnitt, sondern schräg angeschnitten.

C: Übersichtszeichnung, entnommen aus PFLÜGER et al. (1988). Die wichtigsten morphologischen Strukturen in dieser Schnittebene (medianer Sagittalschnitt) sind gezeigt. D: Original-Fotografie eines medianen Sagittalschnittes vom Mesothorakalganglion eines anderen Präparates (lichtmikroskopische Vergrößerung). Die äußere Begrenzung der dorsalen Kommissur DCl ist geringer als die der hier erkennbaren Kommissuren DCIII und DCV und die angeschnittenen DCl-Profile haben einen vergleichsweise geringeren mittleren Durchmesser.

Abkürzungen: a: anterior; aVAC: anterior ventral association centre; d: dorsal; DCI-DCV: dorsal commissures I-V; mVAC: median ventral association centre; $\mathrm{p}$ : posterior; TT: Ttract; v: ventral; VCI-VCIl: ventral commissures I-II; vVAC: ventralmost ventral association centre. Nach PFLÜGER et al. (1988). 
sich teilweise nur schwer zuordnen lässt, welche Fasern der Kommissur entstammen und welche nicht. Auch scheinen die Profile der angeschnittenen Fasern im Mittel einen geringeren Durchmesser zu haben, als die der benachbarten Kommissur DCIII (Abb. 37, A,B und D). Es wurde jedoch hier nicht versucht, diesen Unterschied quantitativ zu bestimmen, da viele Profile der Fasern schräg angeschnitten sind und aus den oben genannten Gründen nicht immer klar ist, welche Fasern der jeweiligen Kommissur und welche den longitudinalen Trakten entstammen. Es lässt sich aber auch schon rein qualitativ erkennen, dass die Fasern der dorsalen Kommissur DCl einen geringeren mittleren Durchmesser haben als die Fasern der dorsalen Kommissur DCIII. 
4 Diskussion

4.1 Allgemeine Erkenntnisse über kommissurale Interneurone im Mesothorakalganglion von Wanderheuschrecken unabhängig von ihrem Verzweigungstyp

Die generellen Erkenntnisse unabhängig vom Typ der hier vorgestellten kommissuralen Interneurone mit Eigenschaften von einer die Extremitäten koordinierenden Funktion sind folgende:

1. Viele Interneurone (im Folgenden als IN abgekürzt), die man mit der Glasmikroelektrode im Bereich der dorsalen Kommissuren penetrieren kann, scheinen eine die Extremitäten koordinierende Funktion zu haben. Aber oftmals sind sie sehr groß und verzweigen auch intersegmental, so dass sie vielleicht allgemeinere Funktion im Kontext der Koordination mehrerer Extremitäten haben und nicht nur entweder bilateral oder intersegmental koordinieren.

2. Eine zuvor erwartete funktionale Spezifität unter den kommissuralen IN bei der Koordination der Extremitäten wurde nicht gefunden. Oftmals sind große, intersegmentale IN mit Eigenschaften einer bilateral koordinierenden Funktion anscheinend auch im Kontext von intersegmentalen Reflexen aktiv, die entsprechend durch taktile Reizung an der Dorsalseite der Vorderbeintarsen oder sogar von Haaren an Extremitäten und lateral am Thorax/Abdomen ausgelöst werden.

3. In vielen Fällen haben intersegmentale IN, die an der Koordination der Extremitäten untereinander beteiligt sein könnten, ein ventral gelegenes Soma, wohingegen Verzweigungs- und lokale Projektionsbereiche in der Regel dorsal oder zumindest auf intermediärem bis dorsalem Niveau gelegen sind. So lassen sich häufig Verzweigungen (Kollaterale des Axons, aber auch Verzweigungen vom primären Neuriten) in der Region lateraler Neuropile (aLAC, pLAC; Nach PFLÜGER et al., 1988) und longitudinaler Trakte (vor 
allem MDT, LDT, DIT und DMT) finden. Auch Verzweigungen in der Region von ventralen Iongitudinalen Trakten (VIT, VMT, VLT) und ventralen Neuropilen (mVAC, aVAC) kommen in einem Bereich im Ganglion vor, in dem diese Strukturen eher intermediär lokalisiert sind (siehe Abb. 4-5). Seltener erstrecken sich Ausläufer vom primären Neuriten bis in den Bereich von größtenteils weiter ventral gelegenen Strukturen wie beispielsweise der ventralen Neuropile IVAC und vVAC oder des ventralen Traktes MVT.

4. Es lässt sich kein Zusammenhang zwischen Verzweigungstyp eines kommissuralen IN (Typ CIN-I oder Typ CIN-II Neurone) und der dorsalen Kommissur, durch die es läuft, erkennen. Innerhalb aller dorsalen Kommissuren DCl-DCVI sind bei intrazellulären Ableitungen beide Typen anzutreffen. Auch scheint es keinerlei Präferenzen zu geben, nach welchen IN der weiter anterior gelegenen dorsalen Kommissuren DCI-DCII häufiger ein aufsteigendes (oder absteigendes) Axon haben, nach welchen IN der medianen Kommissuren DCII-DCIII häufiger etwa ein Axon mit Bifurkation besitzen und Neurone der mehr posterior gelegenen Kommissuren DCIV-DCVI häufiger ein absteigendes (oder aufsteigendes) Axon haben. Alle Typen können in jeder der sechs dorsalen Kommissuren vorgefunden werden.

4.2 Welche Rolle spielen intersegmentale kommissurale Interneurone vom Typ CIN-I bei der bilateralen (intrasegmentalen) Koordination der Extremitäten?

Es gibt einige Forschungsergebnisse, die darauf hinweisen, dass die bilaterale (intrasegmentale) Koordination der Extremitäten auch bei Insekten möglicherweise nicht allein über die mechanische Koppelung erfolgt, sondern dass auch neurale Mechanismen in diesem Zusammenhang von Bedeutung sein können. Für Stabheuschrecken wurde bereits gezeigt, dass die bilaterale Koordination von sich kontralateral gegenüberliegenden Extremitäten zumindest während der Lokomotion auf neurale Mechanismen angewiesen ist (GRAHAM and CRUSE 1981; EPSTEIN and GRAHAM 1983). Wenn man bei gehalterten Stabheuschrecken eine in starkem Maße mechanische Entkoppelung der frei beweglichen Extremitäten beim Laufen 
dadurch erreicht, dass man sie auf einer Quecksilberoberfläche laufen lässt, so zeigen sie dennoch ein weitgehend koordiniertes Schrittmuster (GRAHAM and CRUSE 1981). Das deutet daraufhin, dass auch die bilaterale Koordination der Extremitäten zumindest zum Teil über zentralnervöse Mechanismen erreicht werden muss. HEINRICH und ELSNER (1997) konnten an drei Feldheuschreckenarten zeigen, dass die bilaterale Koordination der Hinterbeine während der Stridulation auch über zentralnervöse Prozesse erreicht wird, wobei die entsprechenden neuronalen Pfade im Falle der schnellen rhythmischen Bewegungen während der Stridulation im Metathorakalganglion (intraganglionär) lokalisiert sind. HUSTERT (1989) hat für Wander- und Wüstenheuschrecken ein intersegmentales kommissurales Interneuron beschrieben, welches ebenfalls Eigenschaften einer bilateral und/oder intersegmental die Extremitäten koordinierenden Funktion hat. All dies deutet darauf hin, dass die lokalen neuronalen Netzwerke, die die motorischen Erregungsmuster für die jeweilige ipsilaterale Extremität erzeugen, durch Interneurone untereinander gekoppelt werden. Im Falle der bilateralen Koordination kommen dafür ausschließlich kommissurale Interneurone in Frage, da die relevante neuronale Information in diesem Kontext ja zwischen zwei sich gegenüberliegenden Hemiganglien über die Mittelinie hinweg übertragen werden muss. Zumindest bei anderen Tiergruppen (vor allem Vertebraten) ist bereits bekannt, dass solche kommissuralen Interneurone an der bilateralen Koordination der Extremitäten (bzw. Muskulatur in den gegenüberliegenden Körperhälften) beteiligt sind (SOFFE et al. 1984; BUCHANAN 1986; BUCHANAN and GRILLNER 1987; BUTT et al. 2002; BUTT and KIEHN 2003; MULLONEY and HALL 2003; LANUZA et al. 2004; BIRÓ et al. 2006, 2008; QUINLAN and KIEHN 2007). Im Falle von Lokomotion (alternierende Bewegungen der Extremitäten bzw. alternierende Muskelkontraktionen in gegenüberliegenden Körperflanken wie beispielsweise beim Fisch) und den in diesem Kontext auftretenden rhythmisch-alternierenden motorischen Erregungsmustern geht man vielfach davon aus, dass die entsprechenden lokalen neuronalen Netzwerke (oder Oszillatoren) über eine reziprok (wechselseitig) gerichtete Hemmung erfolgt (BUCHANAN 1986; BUCHANAN and GRILLNER 1987). Das bedeutet, dass offensichtlich viele der bilateral koordinierenden kommissuralen Interneurone einen inhibitorischen Einfluss auf das kontralaterale neuronale Netzwerk haben (BUCHANAN and COHEN 1982; BUCHANAN 1986; BUCHANAN and GRILLNER 1987; BIRÓ et al. 2008). 
Vor allem bei den hier beschriebenen kommissuralen und intersegmentalen InterNeuronen vom Typ CIN-I gibt es einige Hinweise auf eine mögliche Beteiligung im Kontext der bilateralen Koordination der Mittelbeine. Ihre bilateralen Verzweigungsbereiche sind meistens äußerst weit dorsal und lateral in der Region der lateralen Neuropile (aLAC, pLAC) lokalisiert (CIN-I(ad)c1, Abb. 6; CIN-I(a)c1, Abb. 11; CINI(d)c1, Abb. 14). Zwar wurden im Rahmen der vorliegenden Arbeit keine ultrastrukturellen Analysen der Morphologie der Neurone durchgeführt, so dass über ihre genaue Verschaltung nichts bekannt ist. Zumindest aber verzweigen sie in mutmaßlichen, typischen Regionen, in welchen eine Verschaltung zwischen lokalen, intrasegmentalen sowie intersegmental gerichteten neuronalen Prozessen angenommen werden kann, da bekanntlich auch viele Typen von Mechanosensoren in diese lateralen Neuropile projizieren (zusammenfassender Überblick in: PFLÜGER et al. 1988). So könnten sie also sensorischen Input beispielsweise von Gelenkstellungsrezeptoren, Chordotonal-Organen, campaniformen Sensillen und anderen erhalten. Auch eine Verschaltung von sensorisch integrierenden lokalen Interneuronen (spikende oder nicht-spikende Interneurone: BURROWS 1985, 1987, 1988; BURROWS and SIEGLER 1982, 1984; SIEGLER and BURROWS 1979, 1984; WATSON and BURROWS 1983, 1987a; NAGAYAMA and BURROWS 1990) auf kommissurale Interneurone dieses Typs ist denkbar. Auf der anderen Seite könnten die kommissuralen Interneurone in diesen Regionen entweder direkt auf Motoneurone oder auf andere lokale und intersegmentale Interneurone verschalten, die ihrerseits wiederum die Aktivität der Motoneurone beeinflussen. Denn alle zuletzt genannten (Motoneurone, lokale und intersegmentale Interneurone) verzweigen vorzugsweise ebenfalls in den lateralen Neuropilen (zusammenfassender Überblick in: PFLÜGER et al. 1988).

Viele kommissuralen Interneurone vom Typ CIN-I haben auch im Mesothorakalganglion (in einem oder in beiden Hemiganglien) Verzweigungen, an deren Fasern deutlich erkennbar Varikositäten vorhanden sind. Solche kleinen lokalen Aufschwellungen an den Fasern deuten auf eine mögliche lokale Outputregion hin, da gerade an ihnen oftmals Outputsynapsen lokalisiert sind. Bei den bilateral verzweigenden kommissuralen Interneuronen kommen einerseits solche vor, die im ipsilateralen Hemiganglion scheinbar überwiegend nicht-varikose (vermutlich dendritische) Fasern und im gegenüber liegenden Hemiganglion Kollaterale haben, 
die ihrerseits deutlich erkennbar varikose Verzweigungen haben und bei welchen es sich sehr wahrscheinlich um lokale Neben-Projektionsbereiche im Mesothorakalganglion handelt (CIN-I(d)c1, Abb. 14; CIN-I(d)c2, Abb. 18). Es gibt aber auch solche Typ CIN-I Neurone, die entweder nur in einem oder in beiden Hemiganglien sowohl eher dendritische als auch varikose Fasern haben, wo also in entsprechenden Verzweigungsbereichen Input- und Outputregionen möglicherweise nebeneinander vorkommen ( $\mathrm{CIN}-\mathrm{I}(\mathrm{ad}) \mathrm{c} 1$, Abb. 6; CIN-I(a)c1, Abb. 11). Im Falle von letzteren erscheint es aus zunächst morphologischer Sicht denkbar, dass sie im Zuge einer falls zutreffenden - bilateralen Koordination der Mittelbeine nicht nur unilateral, sondern bilateral auf Motoneurone in beiden Hemiganglien verschalten könnten. Unter der Voraussetzung, dass es sich bei den beobachteten varikosen Fasern der bilateralen Verzweigungsbereiche auch tatsächlich um lokale (bilateral lokalisierte) Outputregionen handelt, könnte das bedeuten, dass solche Neurone eine für beide Mittelbeine gleichermaßen koordinierende Funktion haben, möglicherweise unabhängig davon, ob ein bilateraler Reflex in der einen oder der anderen Richtung abläuft (mögliche bilateral-bidirektional koordinierende Funktion beider Mittelbeine). Bei solchen kommissuralen CIN-I Neuronen, die anscheinend eher in bilateral voneinander getrennten Verzweigungsbereichen Input auf einer Seite erhalten und in das kontralaterale Hemiganglion projizieren (CIN-I(d)c1, Abb. 14; CIN-I(d)c2, Abb. 18), ist hingegen - falls eine bilaterale Koordination zutrifft - eher eine entsprechend bilateral-unidirektionale Koordination zu erwarten, abhängig von der Richtung, in welcher ein bilateraler Reflex abläuft. Die physiologischen Befunde zu den einzelnen Neuronen dieser Gruppe (Typ CIN-I) sprechen in vielen, aber nicht in allen Aspekten für diese Vermutungen.

Die neuronalen Aktivitäten der beschriebenen Typ CIN-I Neurone korrelieren in den meisten Fällen strenger mit einer oder beiden während der Experimente parallel gemessenen Aktivitäten der Depressor trochanteris Muskeln als dies bei den meisten kommissuralen Neuronen vom Typ CIN-II der Fall ist. So zeigt sich meistens eine neuronal erhöhte Aktivität durch phasische Bursts, die zeitlich entweder klar alternierend (CIN-I(a)c1, Abb. 13; CIN-I(d)c2, Abb. 20-21) oder deutlich synchron (CIN-I(ad)c1, Abb. 8-10; CIN-I(d)c1, Abb. 16-17) zu mindestens einer der auftretenden Muskelaktivitäten erscheint, jedoch nicht etwa reaktiv auf eine taktile Reizung am Tarsus einer der beiden Mittelbeine, wenn in diesem Kontext keine 
Muskelaktivität erscheint. Die beiden Neurone (CIN-I(a)c1, CIN-I(d)c2), die eine neuronal erhöhte Aktivität alternierend zur Aktivität des kontralateralen Depressormuskels zeigen, haben möglicherweise auch eine potentielle Outputregion (Kollaterale mit überwiegend varikosen Fasern) im kontralateral zum Soma gelegenen Hemiganglion in der Region der lateralen Neuropile ( $\mathrm{CIN}-\mathrm{I}(\mathrm{a}) \mathrm{c} 1, \mathrm{Abb} .11$; $\mathrm{CIN}-\mathrm{I}(\mathrm{d}) \mathrm{c} 2$, Abb. 18). Hier besteht die Möglichkeit, dass sie möglicherweise entweder einen (direkt oder indirekt) inhibitorischen Einfluss auf die Aktivität des kontralateralen Depressors haben oder auf synergistische Muskeln in derselben Extremität haben oder dass sie einen (ebenso direkt oder indirekt) erregenden Einfluss auf den kontralateralen Flexormuskel (oder entsprechende Synergisten im selben Bein) ausüben. Über die genaue Verschaltung ist bei keinem der hier gezeigten kommissuralen Neurone etwas bekannt. Hyper- und Depolarisation des Membranpotentials des abgeleiteten Neurons hatten zwar die erwarteten Effekte bezogen auf die Neuronaktivität (Frequenzsteigerung bei Depolarisation und Erniedrigung bei Hyperpolarisation), wirkten sich jedoch bei keinem Präparat deutlich erkennbar auf eine der Muskelaktivitäten (gemessen oder visuell wahrgenommene Muskelkontraktionen) aus. Das spricht daher zumindest nicht für eine erregende direkte Verschaltung von kommissuralen Interneuronen vom Typ CIN-I auf Motoneurone in einem der Hemiganglien. Jedoch besteht die Möglichkeit, dass solche kommissuralen Interneurone einen inhibitorischen Einfluss auf bestimmte Muskeln des kontralateralen Beins ausüben. Denn bei Wirbeltieren (SOFFE et al. 1984; BUTT et al. 2002; BUTT and KIEHN 2003; LANUZA et al. 2004; BIRÓ et al. 2006, 2008; QUINLAN and KIEHN 2007) und auch bei Invertebraten (Krebs: MULLONEY and HALL 2003) weiß man, dass zum Beispiel die rhythmisch alternierenden motorischen Erregungsmuster, die zum Beispiel beim Laufen und während der undulatorischen Schwimmbewegungen von Neunaugen auftreten, durch reziprok gerichtete Inhibition zwischen den lokalen und hemisegmental gekoppelten neuronalen Netzwerken (Oszillatoren) zustande kommen. Diese reziprok gerichtete Hemmung wird, wie oben beschrieben, auf kommissurale Interneurone zurückgeführt, die im kontralateralen Hemisegment Inhibition vermitteln (BUCHANAN and COHEN 1982; BUCHANAN 1986; BUCHANAN and GRILLNER 1987; BIRÓ et al. 2008). Daher besteht die Möglichkeit, dass auch kommissurale Interneurone im Nervensystem von Wanderheuschrecken an der bilateralen Koordination der Extremitäten beteiligt sind und zwischen kontralateral 
korrespondierenden lokalen neuronalen Netzwerken für Lokomotion inhibitorisch verschalten. Denn auch für Wanderheuschrecken gilt, dass ihre Extremitäten während Laufbewegungen in einem alternierenden tripod gait Schrittmuster koordiniert werden (BURNS 1973). Dabei befinden sich die kontralateral benachbarten Mittelbeine nicht synchron, sondern alternierend in der Stemmphase. Somit ist auch hier eine reziprok gerichtete Hemmung der lokalen neuronalen Netzwerke für Lokomotion in beiden Hemiganglien zu erwarten. Möglicherweise geschieht dies bei Wanderheuschrecken ebenfalls über kommissurale, bilateral die Extremitäten koordinierende Interneurone wie die hier beschriebenen vom Typ CIN-I.

Das Neuron CIN-I(d)c2 ähnelt sowohl von der morphologischen Struktur her (Abb. 18) als auch aufgrund der physiologischen Eigenschaften (Abb. 20-21) dem Interneuron 325, das von RAMIREZ und PEARSON (1988) beschrieben wurde und das Eigenschaften einer die Extremitäten koordinierenden Funktion hat, da es während Laufbewegungen rhythmische neuronale Aktivität zeigt und während des Fluges inaktiv ist. Da das betreffende Präparat keine Laufbewegungen äußerte, ist nicht klar, ob auch das Neuron CIN-I(d)c2 im Kontext von Lokomotion rhythmisch aktiv ist. Aber das Neuron zeigte bereits beim ruhenden Präparat (keine Reizung und auch keine erkennbaren Bewegungen) eine tonische Spontanaktivität, die fast schon rhythmisch erscheint, da immer kurze Salven von 2-3 Spikes auftreten, die durch kurze inaktive Intervalle etwas voneinander abgesetzt waren (Abb. 20-21). Zwar wurde die neuronale Aktivität während einer passiven Anhebbewegung vom ipsilateralen Mittelbein nicht durch kontinuierlich hemmende Einflüsse auf die Spikefrequenz verringert, jedoch verstärkte sich dabei noch der rhythmische Charakter der neuronalen Aktivität (längere Bursts von Aktionspotentialen bei höheren Frequenzen und verlängerte inaktive Intervalle zwischen den Bursts). Es gibt also zumindest Hinweise dafür, dass auch das Neuron CIN-I(d)c2 eine im Kontext von Lokomotion bilateral die Mittelbeine koordinierende Funktion haben könnte. Wenn das zutrifft, handelt es sich dabei wahrscheinlich um eine bilateralunidirektional koordinierende Funktion, bei welcher sensorisch relevante Information über Bewegungen und veränderte Zustände der ipsilateralen Extremität zur Koordination des kontralateralen Mittelbeins genutzt wird. Denn varikose Fasern (und damit eine potentielle Outputregion) kommen bei diesem Neuron im Mesothorakalganglion nur an den Neben-Kollateralen im kontralateralen Hemiganglion vor, 
während die ipsilateralen Verzweigungen eher von dendritischem Charakter zu sein scheinen (nicht-varikos). Beim Neuron CIN-I(a)c1 liegt eine solche klare Trennung von vermutlichen Input- und Outputregionen nicht vor (Abb. 11). Zwar sind auch hier die kontralateral gelegenen Neben-Kollaterale im Mesothorakalganglion überwiegend von varikosem Charakter, daneben kommen aber auch bei den im ipsilateralen Hemiganglion gelegenen Verzweigungen varikose Fasern vor, die nebeneinander mit nicht-varikosen Verzweigungen auftreten. Wenn das Neuron CIN-I(a)c1 also auch in die bilaterale Koordination der Mittelbeine involviert ist, so scheint dies nach einem komplexeren Prinzip zu erfolgen. Möglicherweise kann das Neuron eine für beide Mittelbeine gleichermaßen bilateral koordinierende Funktion haben (bilateralbidirektional koordinierende Funktion), da potentielle lokale Projektionsbereiche in beiden Hemiganglien im Bereich der lateralen Neuropile vorzukommen scheinen. Ein weiterer Hinweis auf eine möglicherweise starke bilaterale Koppelung zwischen beiden Mittelbeinen könnte beim Neuron CIN-I(a)c1 dadurch gegeben sein, dass mehrere rekurrente Fasern separat über verschiedene dorsale Kommissuren in das jeweils kontralaterale Hemiganglion ziehen und dort mit lokalen Verzweigungen überlappen. Bei diesem Präparat traten gelegentlich rhythmische synchrone Kontraktionen beider Depressormuskeln auf, während derer das Neuron CIN-I(a)c1 auch eine rhythmische, streng zu den Aktivitäten dieser beiden Muskeln korrelierte neuronale Aktivität zeigte (Abb. 13, B). Auffällig ist dabei, dass dieses rhythmische neuronale Erregungsmuster offensichtlich mit einer Modulation der Spikeamplitude einhergeht, deren Ursprung aufgrund der Datenlage unklar bleibt (siehe Bemerkungen dazu in der Kurzdiskussion zum Neuron CIN-I(a)c1 und auch CIN$\mathrm{I}(\mathrm{d}) \mathrm{c} 2$ ). Möglicherweise ist dies auf einen noch genauer zu untersuchenden Effekt zurückzuführen, der bei großen intersegmentalen Interneuronen, die über mehrere Spike-generierende Zonen verfügen können (HUSTERT 1985), mitunter auftreten kann. Es ist aber auch nicht auszuschließen, dass dieses Phänomen möglicherweise sogar in direktem Zusammenhang mit einer die Extremitäten koordinierenden Funktion eines Neurons steht. Im Falle vom Neuron CIN-I(a)c1 erschienen solche in der Amplitude modulierten Spikes jedenfalls ausschließlich im Kontext der beschriebenen rhythmischen Neuronaktivität während der spontanen rhythmischsynchronen Muskelzuckungen beider Depressoren (Abb. 13, B) und zwar in streng alternierend zur Aktivität des kontralateralen Depressors korrelierten und deutlich abgegrenzten Phasen. 
Das Neuron CIN-I(d)c1 könnte einen (direkt oder indirekt) erregenden Einfluss auf den kontralateralen Depressormuskel haben, da neuronal erhöhte Aktivität immer unmittelbar vor der Aktivität dieses Muskels beginnt, eine Weile synchron dazu andauert, aber immer vor deren Beendigung abklingt (Abb. 16-17). Als stützendes Argument dafür kommt hinzu, dass das Neuron im kontralateralen Hemiganglion eine Nebenkollaterale trägt, die in die lateralen Neuropile projiziert und deren Fasern deutlich erkennbar varikos sind (Abb. 14). Wenn dieses Neuron also in die bilaterale Koordination der Extremitäten involviert ist, so kann eine bilateral-unidirektional koordinierende Funktion angenommen werden. Dabei könnten sensorische Informationen über Bewegungen und veränderte Zustände des ipsilateralen Mittelbeins auf die nicht-varikosen Verzweigungen in den lateralen Neuropilen des ipsilateralen Hemiganglions übertragen werden (vermutlich lokale Inputregion des Neurons) und zur bilateralen Koordination des kontralateralen Mittelbeins über varikose Neben-Kollaterale des Neurons (mutmaßliche Outputregion) im kontralateralen Hemiganglion genutzt werden.

Auch beim Neuron CIN-I(ad)c1 (Abb. 8-10) spricht einiges für eine Beteiligung an der Koordination der Extremitäten. Ebenso wie bei den anderen Neuronen vom Typ CINI erscheint eine neuronale Aktivität, die deutlich synchron mit beiden parallel gemessenen Muskelaktivitäten der Depressoren korreliert ist. Aber im Gegensatz zu den zuvor beschriebenen Neuronen wird das Neuron CIN-I(ad)c1 unidirektional gehemmt, nämlich immer dann, wenn stärkere Muskelaktivität des kontralateralen Depressormuskels (sowohl durch taktile kontralaterale Reizung ausgelöst als auch während spontan auftretender Muskelaktivität) erscheint. Das Neuron CIN-I(ad)c1 besitzt ein Axon, das im kontralateralen Konnektiv eine Bifurkation hat, das also auch noch ins Pro- und Metathorakalganglion projiziert. Wenn dieses Neuron drei Extremitäten (das ipsilaterale Mittelbein und das kontralaterale Vorder- und Hinterbein) im Sinne eines alternierenden tripod gait Schrittmusters koordinieren (koaktivieren) würde, was aufgrund seines Verzweigungsmusters möglich wäre, so wäre seine Hemmung sinnvoll, wenn an einem dieser drei von inm möglicherweise koordinierten Extremitäten eine externe „Störung“ (beispielsweise durch passives Anheben) auftritt und entsprechend eine Verlagerung auf die anderen drei Extremitäten des alternierten tripod gait erfolgen muss. Auch HUSTERT (1989) hat für ein kommissurales intersegmentales Interneuron mit einem vergleichbaren 
Projektionsbereich (kontralateral durchlaufendes Axon) und ähnlichen physiologischen Merkmalen zu dem hier beschriebenen Neuron CIN-I(ad)c1 eine ähnliche Funktion bezüglich der bilateralen/intersegmentalen Koordination der Extremitäten während des alternierenden tripod gait Schrittmusters angenommen und ein Modell einer exzitatorisch wirkenden Koppelung zwischen dem ipsilateralen Mittelbein und dem kontralateralen Vorder- und Hinterbein vorgeschlagen.

Für eine starke bilaterale Koppelung zwischen beiden Hemiganglien spricht beim Neuron CIN-I(ad)c1 außerdem sein komplexes Verzweigungsmuster: Es besitzt zahlreiche rekurrente Fasern in beiden Hemiganglien, die jeweils über separate dorsale Kommissuren über die Mittellinie ziehen und dort mit lokalen Verzweigungen überlappen. Auch der primäre Neurit selbst durchquert die dorsale Kommissur DCI zweimal.

Alle vier bisher besprochenen Neurone vom Typ CIN-I (CIN-I(ad)c1, CIN-I(a)c1, CIN$\mathrm{I}(\mathrm{d}) \mathrm{c} 1$, CIN-I(d)c2) haben ein kontralaterales Axon, das entweder im Konnektiv aufsteigt (Abb. 11), absteigt (Abb. 14, 18) oder durchläuft (Abb. 6). Alle vier projizieren also auch noch ins Pro- oder Metathorakalganglion (oder in beide gleichzeitig), so dass bei ihnen neben einer potentiellen bilateralen Koordination zwischen den Mittelbeinen auch noch eine Funktion im Kontext der intersegmentalen Koordination der Extremitäten in Betracht zu ziehen ist. Das betrifft auch alle in der vorliegenden Arbeit beschriebenen Neurone vom Typ CIN-II mit medianem Hauptverzweigungsbereich. Es stellt sich die damit die Frage, ob eine Integration von bilateraler und intersegmentaler Koordination der Extremitäten durch kommissurale und intersegmentale Interneurone vielleicht für Insekten vorteilhaft ist, die ihre Extremitäten bei der Lokomotion in charakteristischen Schrittmustern wie zum Beispiel dem so genannten tripod gait (Dreipunkt-Unterstützung) koordinieren. Wenn einzelne Interneurone in der Lage wären, sowohl bilateral benachbarte Extremitäten, als auch ipsilateral (intersegmental) benachbarte Extremitäten gleichzeitig zu koordinieren, könnte dies in der Tat vorteilhaft sein, da zum Beispiel beim tripod gait Schrittmuster, welches ja auch Wanderheuschrecken bei der Lokomotion nutzen, immer drei Extremitäten (ein Mittelbein und gleichzeitig das kontralaterale Vorderund Hinterbein) synchron gegen den Untergrund gestemmt werden müssen. Im Falle einer Störung auch nur einer dieser drei sich während der Lokomotion gerade in der 
Stemmphase befindlichen Extremitäten ist eine möglichst schnelle Verlagerung auf die Extremitäten des alternativen tripod gait notwendig. Je weniger synaptische Verschaltungen dazu notwendig sind, desto schneller könnten die relevanten neuronalen Informationen dabei zwischen den lokalen neuronalen Netzwerken, die jeweils das motorische Erregungsmuster für die ipsilaterale Extremität erzeugen, übertragen werden. Auch schnelle mechanosensorische Verschaltungswege, wie sie beispielsweise die an Trochanter und Coxa befindlichen campaniformen Sensillen leisten (HÖLTJE and HUSTERT 2003), wären in diesem Zusammenhang vorteilhaft. Diese genannten campaniformen Sensillen projizieren auch dort in die lateralen Neuropile (aLAC, pLAC: PFLÜGER et al. 1988), wo auch alle hier vorgestellten kommissuralen Interneurone vom Typ CIN-I verzweigen.

\subsection{Welche Rolle spielen intersegmentale kommissurale Interneurone vom Typ CIN-II bei der bilateralen (intrasegmentalen) Koordination der Extremitäten?}

Eine bilaterale Koordination der Mittelbeine scheint bei den kommissuralen Interneuronen vom Typ CIN-II im Mesothorakalganglion nicht die primäre Funktion zu sein. Zwar haben sie meistens Eigenschaften, die eine allgemeinere Funktion im Kontext der (bilateralen und intersegmentalen) Koordination der Extremitäten möglich erscheinen lassen, aber gegen eine zumindest direkte bilaterale Koordination bei den Bewegungen der Mittelbeine spricht schon allein ihr morphologisches Erscheinungsbild. Dieses ist dadurch gekennzeichnet ist, dass die Neurone vom Typ CIN-II median gelegene Hauptverzweigungsbereiche haben und ihre Verzweigungen nicht bilateral in beiden Hemiganglien bis an die lateralen Neuropile heranreichen, was aber bei einer direkt bilateral die Mittelbeine koordinierenden Funktion zu erwarten wäre. Denn es müsste sensorische Information über Bewegungen und aktuell veränderte Zustände mindestens bezüglich einer Extremität auf ein bilateral koordinierendes Neuron übertragen werden, dass dann seinerseits eine koordinierende Funktion auf das im kontralateralen Hemiganglion befindliche lokale neuronale Netzwerk ausüben könnte. Aber zum einen projizieren die meisten Mechanosensoren (Extero- und Propriozeptoren) ipsilateral in die ventralen und lateralen Neuropile (PFLÜGER et al. 1988), zum anderen verschalten auch eine Vielzahl der Neurone (Moto-, lokale und 
intersegmentale Interneurone und andere), die Teil des neuronalen Netzwerkes sind, hauptsächlich in diesen lateral-dorsal gelegenen Neuropilregionen (TYRER and ALTMAN 1974; ALTMAN and TYRER 1977; ALTMAN 1980; SIEGLER and BURROWS 1979, 1984; BURROWS and SIEGLER 1982, 1984; WATSON and BURROWS 1983, 1987a; WATSON 1984; BURROWS 1985, 1987, 1988; NAGAYAMA and BURROWS 1990). Eine Beteiligung der kommisuralen Interneurone vom Typ CIN-II an der bilateralen Koordination der Mittelbeine auf indirektem Wege über eines oder mehrere zwischengeschaltete Interneurone (lokale und/oder andere intersegmentale IN) erscheint durchaus denkbar. Dies lässt sich aber anhand der vorliegenden morphologischen Daten und wegen der komplexeren Physiologie dieser Neurone nicht klar entscheiden. Zwar zeigen auch die kommissuralen Interneurone vom Typ CIN-II oftmals eine neuronale Aktivität, die mit einer oder beiden parallel gemessenen Aktivitäten der Depressor trochanteris Muskeln korreliert ist (sowohl nach Reflexen, die durch die taktilen Reize an den Mittelbeintarsen ausgelöst wurden, als auch während gelegentlich spontan aufgetretenen rhythmisch-synchronen Muskelzuckungen beider Depressormuskeln). Aber diese Korrelation ist oftmals keine strenge, sondern eine etwas variablere. Dabei bleibt der Grundcharakter der zeitlichen Korrelation zur Muskelaktivität (alternierende oder synchrone Korrelation zur Muskelaktivität) meistens erhalten. Aber das genaue timing (die genaue Phasenlage) vom neuronalen Burst zur Phase der Muskelaktivität kann sich in gewissen Grenzen sogar innerhalb einer Aufzeichnung bei zum Beispiel mehrfach wiederholten Einzelreizen ändern. So erscheint zum Beispiel im Falle einer synchronen Korrelation neuronale Aktivität manchmal einige Millisekunden vorher (präaktive Phase des Neurons), im späteren Verlauf dann genau synchron und manchmal sogar geringfügig verzögert mit postaktiver Phase der neuronalen Aktivität. Von den hier genauer beschriebenen Neuronen vom Typ CIN-II betrifft das die Neurone CIN-II(ad)c1 (Abb. 24) und CINII(a)i1 (Abb. 31-32), bei anderen hier nicht näher beschriebenen Neuronen dieses Typs war dies aber manchmal sogar noch deutlicher der Fall. Neben dieser geringfügigen zeitlichen Variabilität der Korrelation der neuronalen Aktivität zur Muskelaktivität erschienen oft auch komplexere neuronale Aktivitätsmuster als dies in der Regel bei den bilateral verzweigenden Neuronen vom Typ CIN-I der Fall war. So führten taktile Reizungen jeweils am Tarsus des kontralateralen Mittelbeins bei den beiden Neuronen $\mathrm{CIN}-\mathrm{II}(\mathrm{ad}) \mathrm{c} 1$ und $\mathrm{CIN}-\mathrm{II}(\mathrm{a}) \mathrm{i} 1$ zunächst zu einer präaktiv oder 
synchron zu einer der Muskelaktivitäten erscheinenden neuronalen Erregung, während einer synchron auftretenden erhöhten Muskelaktivität beider Depressoren führten diese jedoch zu einer Hemmung des Neurons für die Dauer dieser Phase. Im Anschluss an die beendeten synchronen Muskelaktivitäten setzte bei beiden Neuronen eine dauerhaft erhöhte neuronale Phase der Erregung ein, die über mehrere Sekunden andauerte (Abb. 24, A; Abb. 32, C). Die gehemmte Phase des Neurons trat nur während synchron erhöhter Muskelaktivität beider Depressoren, jedoch nicht während erhöhter Aktivität in nur einem Depressormuskel auf. Eine mögliche Erklärung hierfür wäre, dass betreffende Neurone an der intersegmentalen und/oder bilateralen Koordination der Extremitäten während der Lokomotion zumindest auf indirektem Wege beteiligt sind. Während der Lokomotion bei Wanderheuschrecken werden die Extremitäten in einem alternierenden tripod gait Schrittmuster koordiniert (BURNS 1973). Dabei befinden sich die gegenüber liegenden Mittelbeine nicht gleichzeitig in der Stemmphase. Wenn ein kommissurales Interneuron die Mittelbeine bilateral im Kontext solcher alternierenden Bewegungen koordiniert, so wäre die Hemmung eines solchen Neurons in anderen Verhaltenskontexten zu erwarten, in denen die Mittelbeine synchron bewegt werden. Die Neurone CIN-II(ad)c1 und CIN-II(a)i1 wurden in einem solchen Kontext von synchron auftretenden Muskelkontraktion beider Depressoren in dieser Phase gehemmt (Abb. 24, A; Abb. 32, C), so dass sie möglicherweise an der Koordination der Extremitäten und der Aufrechterhaltung eines alternierenden tripod gait Schrittmusters während der Lokomotion beteiligt sind. Dafür spricht weiterhin, dass alle Neurone vom Typ CIN-II (genau wie alle vom Typ CIN-I) große intersegmentale Interneurone sind, die auch noch ins Pro- oder Metathorakalganglion oder sogar in beide benachbarten Thorakalganglien projizieren und damit auch eine potentiell intersegmental jeweils ipsilateral benachbarte Extremitäten koordinierende Funktion angenommen werden kann. Bei Stabheuschrecken ist zum Beispiel bekannt, dass die Koppelungsstärke zwischen ipsilateral benachbarten Beinpaaren zweimal so stark ist wie zwischen kontralateral benachbarten Beinpaaren (DÜRR 2005). Bei innen wurden einige Regeln für die Koordination der Extremitäten während der Laufbewegungen postuliert (CRUSE 1990 (Review), 1995, 1998). Die drei wichtigsten davon beziehen sich auf intersegmental gerichtete Mechanismen der Koordination zwischen jeweils ipsilateral benachbarten Beinpaaren. 
Zwei Neurone dieser Klasse CIN-II, die Neurone CIN-II(a)i1 (Abb. 29) und CINII(a)i2 (Abb. 35), haben ein im ipsilateral zum Soma gelegenen Konnektiv aufsteigendes Axon. Das Neuron CIN-II(a)i1 ähnelt von der morphologischen Gestalt her einem Interneuron, das WATSON und BURROWS (1983) beschrieben haben und welches auch eine Funktion in anderen Funktionskreisen (beispielsweise des Fluges) zu haben scheint, da es unter anderem auch auf taktile Reizung an den Vorderflügeln und während Auslenkung einer der beiden Vorderflügel phasisch neuronal korrelierte Aktivität zeigt. Aufgrund der beschriebenen Variabilität der neuronalen Aktivitäten der Typ CIN-II Neurone bezüglich der zeitlichen Korrelation zu den gemessenen Depressor trochanteris Muskelaktivitäten und auch wegen ihrem gelegentlich komplexeren neuronalen Erregungsmuster ist es denkbar, dass die kommissuralen Interneurone dieses Typs möglicherweise eher allgemeinere integrierende Funktion im Kontext der Koordination der Extremitäten haben und vielleicht auch Teil von verschiedenen neuronalen Netzwerken aus verschiedenen Funktionskreisen und Verhaltenskontexten sein können. Um dies jedoch entscheiden zu können, wäre unter anderem eine genaue Kenntnis über die Verschaltungen solcher Neurone auf ultrastrukturellem Niveau erforderlich. In allen Experimenten wurde versucht, die Aktivität eines abgeleiteten Neurons durch Hyper- oder Depolarisation seines Membranpotentials entsprechend $\mathrm{zu}$ beeinflussen. Jedoch wirkte sich dies bei keinem der hier beschriebenen Neurone erkennbar auf eine der parallel registrierten Muskelaktivitäten aus. Da eine durch Depolarisation des Membranpotentials erzeugte, erhöhte neuronale Erregung keinen erkennbaren Einfluss auf eine der Muskelaktivitäten hatte (entweder eine der aufgezeichneten, oder eine andere, die sich durch entsprechende Muskelkontraktionen hätte bemerkbar machen können), ist im Falle des abgeleiteten Neurons zumindest keine direkt erregende Verschaltung auf Motoneurone in einem der Hemiganglien anzunehmen. Aber es besteht dennoch die Möglichkeit, dass ein solches Neuron an der bilateralen Koordination der Extremitäten (indirekt) beteiligt sein kann, nämlich dann, wenn es einen inhibitorischen Einfluss entweder auf bestimmte Muskeln oder zum Beispiel auf die Aktivität eines lokalen neuronalen Netzwerks ausübt. Bei Wirbeltieren (Neunaugen: BIRÓ et al. 2006, 2008; Nagetiere: BUTT et al. 2002; BUTT and KIEHN 2003; LANUZA et al. 2004; QUINLAN and KIEHN 2007) und einigen Invertebraten (Krebs: MULLONEY and HALL 2003; Krallenfrosch: SOFFE et al. 1984) ist bekannt, dass kommissurale, an der bilateralen Koordination der 
Extremitäten oder von Muskelkontraktion bilateraler Körperpartien beteiligte Interneurone häufig inhibitorischer Art sind. Dabei werden die rhythmisch alternierenden motorischen Erregungsmuster des zentralen Mustergenerators auf reziprok gerichtete inhibitorische Verschaltungen zwischen den hemisegmental angeordneten lokalen neuronalen Netzwerken (Oszillatoren) zurückgeführt, welche über kommissurale Interneurone vermittelt werden (BUCHANAN 1986; BUCHANAN and GRILLNER 1987; BIRINYI et al. 2003; BIRÓ et al. 2008). Daher besteht die Möglichkeit, dass auch kommissurale Interneurone im Nervensystem von Insekten, von welchen höchstwahrscheinlich auch einige an der bilateralen Koordination der Extremitäten beteiligt sind, inhibitorisch auf lokale Neurone im kontralateralen Hemiganglion verschalten.

Wenn den kommissuralen Neuronen vom Typ CIN-II aber auch keine direkt bilateral die Mittelbeine koordinierende Funktion zuzuordnen ist, so besteht zumindest die Möglichkeit, dass sie indirekt daran beteiligt sein können. Viele von der Gesamtzahl der erfolgreich gefärbten kommissuralen Interneurone waren solche mit median gelegenem Hauptverzweigungsbereich vom Typ CIN-II. Und nicht selten tendieren solche Neurone dazu, dass sie bilateral in beiden Hemiganglien in nächster Umgebung der dorsalen Kommissuren verzweigen (Abb. 34-36: CIN-II(ad)c1, CINII(ad)c2, CIN-II(ad)c4, CIN-II(ad)c5, CIN-II(a)i1, CIN-II(d)c2). Das trifft besonders auf solche Neurone zu, deren Axon im kontralateralen Konnektiv eine Bifurkation hat. Sie haben oftmals einen primären Neuriten, dessen Verzweigungen sich zu beiden Seiten unweit der Mittellinie fortsetzen (Abb. 34-36: CIN-II(ad)c1, CIN-II(ad)c4, CIN$\mathrm{II}(\mathrm{ad}) \mathrm{c5}$ ) oder welcher selbst die dorsalen Kommissuren fast schon „ringförmig“ umgibt (Abb. 34: CIN-II(ad)c2). Neuropilregionen in der näheren Umgebung sind das mediane und das anterior gelegene, ventrale Assoziationszentrum (mVAC, aVAC), die aber beide eher auf intermediärem Niveau lokalisiert sind (Abb. 4-5; Nach PLÜGER et al. 1988). Weiter dorsal gelegen verlaufen in diesem Feld einige longitudinale Trakte (MDT, DMT, DIT, VIT und VMT). Dies scheint daher vielleicht kein typisches Verschaltungsgebiet zu sein, etwa wie das der lateralen Neuropile, in welche viele Mechanosensoren projizieren und wo verschiedene lokale, intersegmentale Interneurone und Motoneurone verzweigen. Aber weil viele der gefundenen kommissuralen Interneurone vom Typ CIN-II hier hauptsächlich verzweigen und auch weil sich die Verzweigungsbereiche mancher der bilateral 
verzweigenden Neurone vom Typ CIN-I zumindest zum Teil auch noch bis in diese Regionen erstrecken, kann angenommen werden, dass dieser Bereich im näheren Umfeld der dorsalen Kommissuren möglicherweise eine Art „Schnittstelle“ ist, wo intersegmentale kommissurale Interneurone untereinander und/oder auf lokale bilaterale Interneurone verschalten und wo somit eine Koppelung von bilateral ablaufenden Prozessen bei der Koordination der Extremitäten mit intersegmental ablaufenden Prozessen in diesem Kontext erfolgen könnte.

4.4 Welche Rolle spielen lokale kommissurale Interneurone im Kontext der bilateralen Koordination der Extremitäten?

Die Rolle von lokalen kommissuralen Interneuronen im Mesothorakalganglion von Wanderheuschrecken bei der bilateralen Koordination der Extremitäten bleibt weiterhin unklar. Die meisten erfolgreichen intrazellulären Ableitungen von kommissuralen Interneuronen gelangen bei solchen, die eine der dorsalen Kommissuren DCII-DCV passieren. Aber bei keinem der dort penetrierten und erfolgreich gefärbten Zellen handelte es sich um ein lokales, bilateral verzweigendes Interneuron. Das bedeutet zwar nicht, dass es hier keine gibt, aber es führt zu den Fragestellungen, ob im Ganglion insgesamt möglicherweise nur eine geringere Anzahl solcher kommissuralen lokalen Neurone vorkommt und ob diese vielleicht häufiger durch die am weitesten anterior und/oder posterior lokalisierten dorsalen Kommissuren (DCl und/oder DCVI) oder eine der ventralen Kommissuren verlaufen. Es wurde versucht, auch im Bereich dieser dorsalen Kommissuren (DCI und DCVI) Zellen zu penetrieren. Aber intrazelluläre Ableitungen, die über eine längere Zeit stabil waren, gelangen hier weniger häufig, wenn auch einige der hier gezeigten Neurone durch solche Kommissuren verlaufen (zum Beispiel: CIN-I(ad)c1, Abb.6; CIN-II(d)c1, Abb. 25; CIN-II(ad)c1, Abb. 22; CIN-II(a)i1, Abb. 29).

Eine mögliche Erklärung für diese Schwierigkeiten bei den intrazellulären Ableitungen von DCl- und DCVI-Interneuronen könnte sein, dass viele von diesen Neuronen einen primären Neuriten und/oder ein Axon mit geringerem Durchmesser (zumindest im kommissuralen Bereich) haben und vielleicht auch diese dorsalen Kommissuren selbst nur von wenigen Neuronen durchquert werden. Um diese 
Vermutungen zu überprüfen, wurden mediane Sagittalschnitte des Mesothorakalganglions (Semi- und Ultra-Dünnschnitte in der Region nahe der Mittellinie) für die Elektronenmikroskopie vorbereitet (siehe Abschnitt 3.3). Die Erkenntnisse, die sich dabei ergaben, scheinen diese Vermutung (zumindest im Fall der dorsalen Kommissur $\mathrm{DCl}$ ) zu bestätigen: Bei der lichtmikroskopischen Analyse der Schnittserien (Semi-Dünnschnitte) ließen sich selbst bei Schnitten, die sehr zentral nahe der Mittellinie lokalisiert waren, nur schwer solche finden, in welchen die dorsale Kommissur DCI mit einiger Sicherheit ausfindig gemacht werden konnte. Bei der in der Abbildung 37 gezeigten dorsalen Kommissur handelt es sich mit einiger Wahrscheinlichkeit um die Kommissur DCl. Aber im Vergleich zur benachbarten Kommissur (DCIII) sind die Profile von Axonen oder kommissuralen Fortsätzen von primären Neuriten, die durch die Kommissur DCl verlaufen, häufig nicht genau quer geschnitten, sondern oft schräg. Dadurch setzen sie sich optisch weniger deutlich von zum Beispiel im Längsschnitt mehr oder weniger schräg getroffenen Fasern der longitudinalen Trakte ab, und die dorsale Kommissur lässt sich somit weniger genau eingrenzen. Außerdem haben viele der in diesem Bereich der Kommissur angeschnittenen Profile einen eher geringeren Durchmesser, verglichen mit denen der benachbarten Kommissur DCIII. Es lässt sich nicht für alle Fasern klar zuordnen, welche von ihnen zur dorsalen Kommissur DCl gehören und welche nicht, aber es sind insgesamt erkennbar wenige (es dürfte sich um weniger als 30 handeln). Vorausgesetzt, dass diese Aussagen zutreffen, könnten sie zum Teil etwaige Schwierigkeiten bei intrazellulären Ableitungen von Interneuronen erklären, die durch diese Kommissur verlaufen. Intrazelluläre Ableitungen von Zellen, die in der Kommissur weniger kompakt gebündelt liegen, können schon nach kürzerer Zeit instabil werden, auch abhängig vom Winkel, unter welchem die jeweilige Zelle mit der Glasmikroelektrode penetriert wird, sowie vom Durchmesser des Axons (beziehungsweise des kommissuralen Abschnitts des primären Neuriten). Ein stützendes Argument für diese Situation (Fasern der dorsalen Kommissur DCI haben einen vergleichsweise geringen Durchmesser, sind oft schräg im Schnitt getroffen und liegen weniger kompakt gebündelt innerhalb der Kommissur vor) ist die Tatsache, dass sich die dorsale Kommissur $\mathrm{DCl}$ in die beiden voneinander getrennten Teilkommissuren $\mathrm{dDCl}$ und vDCl aufteilt (siehe Abb.4, Ausschnitt 19; Nach PFLÜGER et al., 1988). Nahe der Mittellinie kreuzen manche Fasern und ziehen vom dorsal gelegenen Teil der Kommissur zum ventral gelegenen und 
umgekehrt.

Es ist anzunehmen, dass lokale kommissurale Interneurone, welche eine spezifischere Funktion im Kontext der bilateralen Koordination zwischen den Mittelbeinen haben könnten, Axone und primäre Neuriten von geringerem Durchmesser haben, da sie ja nicht über weite Strecken interganglionär, sondern nur lokal auf die beiden Hemiganglien beschränkt verzweigen und somit Spikes nicht über extrem weite Entfernungen geleitet werden müssen. Auch bei der hier angewandten Methode, bei welcher die ventral gehalterten Präparate ihre Vorderund Mittelbeine frei auf einer rotierbaren und in der Vertikalebene flexibel gelagerten Laufkugel bewegen können, besteht die Möglichkeit, dass während Stemmbewegungen der Extremitäten gegen den Untergrund (Laufkugel) Kräfte entstehen, die schnell zum Verlust einer intrazellulären Ableitung führen können. Das könnte zum Teil die oben genannten Schwierigkeiten bei intrazellulären Ableitungen von solchen lokalen DCl- oder DCVI-Interneuronen erklären, wenn deren Axone und primäre Neuriten ohnehin einen eher geringeren Durchmesser haben (verglichen mit Axonen und primären Neuriten von großen intersegmentalen Interneuronen).

Eine andere Möglichkeit wäre, dass solche lokalen bilateral die Mittelbeine koordinierenden Interneurone durch ventrale Kommissuren verlaufen. In diesen Regionen wurde jedoch nicht schwerpunktmäßig versucht, Zellen zu penetrieren. Da die Intrazellulärelektrode bei der hier angewandten Methodik von der Dorsalseite her in das Ganglion eingeführt wird, konnten seltener erfolgreich Zellen penetriert und über längere Zeit stabil abgeleitet werden, die in solchen weiter ventral gelegenen Bereichen lokalisiert sind.

Für Wanderheuschrecken im Metathorakalganglion bereits beschriebene Klassen von lokalen Interneuronen, deren primärer Neurit durch ventrale Kommissuren verläuft, sind zum einen solche vom Typ der ventral midline interneurons, (SIEGLER and BURROWS, 1984; BURROWS and SIEGLER, 1984), zum anderen solche vom Typ der antero-lateral interneurons (SIEGLER and BURROWS, 1984). Beiden Typen gemeinsam ist, dass sie im kontralateral zum Soma des betreffenden Neurons gelegenen Hemiganglion Verzweigungsbereiche sowohl in ventralen und dorsalen Neuropilen haben. Sie integrieren zum einen mechanosensorischen Input, welchen die meisten dieser Interneurone hauptsächlich kontralateral zum Soma in den ventralen Neuropilen erhalten, manche aber auch in den dorsalen Neuropilen 
beziehen. Zum anderen verzweigen Interneurone beider Klassen auch kontralateral in den dorsalen Neuropilen, wo Verschaltungen auf Moto- und andere Interneurone zu erwarten sind. Aber vom Typ der antero-lateral interneurons ist bekannt, dass sie auch im ipsilateralen Hemiganglion verzweigen können (in den dorsalen Neuropilen), dass sie also in beiden Hemiganglien bilateral verzweigen und auf beiden Seiten Input- und Outputregionen haben können (SIEGLER and BURROWS, 1984). Besonders letztere kommen also auch für eine potentiell bilateral zwei im Segment gegenüberliegende Extremitäten koordinierende Funktion in Betracht. Allerdings liegen Input- und Outputregionen bei diesen Interneuronen offensichtlich nicht getrennt voneinander in einem oder dem anderen Hemiganglion vor, sie scheinen also mechanosensorischen Input nicht separat auf einer Seite zu erhalten und dann unidirektional im kontralateralen Hemiganglion (erregend oder hemmend) auf Motound/oder Interneurone zu verschalten. Ein solcher Typ von bilateral verzweigenden und unilateral auf Motoneurone (erregend oder hemmend) verschaltenden lokalen Interneuron mit Eigenschaften einer bilateral die Extremitäten koordinierenden Funktion wurde für Wanderheuschrecken bislang noch nicht beschrieben. Dennoch besteht zumindest die Möglichkeit, dass solche Neurone existieren. Unter der Annnahme, dass ein solcher hypothetischer Neurontyp im Kontext eines bilateralen Reflexes unidirektional erregend auf Motoneurone verschaltet, ist es eher unwahrscheinlich, dass solche Neurone dann durch eine ventrale Kommissur verlaufen, da ihr unilateraler Projektionsbereich ja in den dorsal gelegenen lateralen Neuropilen zu erwarten ist. Im Sinne einer schnellst möglichen Übertragung neuronaler Information für schnelle Reflexe wäre die kürzeste Entfernung die am besten geeignetste. Für einen Interneurontyp, der mechanosensorischen Input beispielsweise ipsilateral in den ventralen Neuropilen von den dorthin projizierenden Mechanosensoren taktiler Haare (PFLÜGER 1980; PFLÜGER et al. 1981) erhalten würde, müsste dementsprechend eine kürzere Strecke durch das Ganglion postuliert werden, die in einem eher intermediärem Bereich verläuft. Da jedoch die meisten Fasern die Mittellinie in der Regel über Kommissuren überqueren, kommen dafür besonders die dorsalen Kommissuren DCI, DCIII, DCIV und DCVI in Frage, da diese nicht so weit dorsal liegen wie die Kommissuren DCII und DCV (siehe Abb. 4-5). Besonders diejenigen Fasern, die die getrennten Faserbündel (dDCl und vDCl) der dorsalen Kommissur DCl kreuzen, scheinen sich nach Durchqueren der Kommissur relativ schnell in tiefere Regionen im Ganglion fortzusetzen. Demnach wäre es 
denkbar, dass manche lokale, bilateral die Extremitäten koordinierende, kommissurale Interneurone durch genau diese Bündel der dorsalen Kommissur DCl verlaufen. Aber aufgrund der beschriebenen Schwierigkeiten bei intrazellulären Ableitungen von DCl-Interneuronen und der Tatsache, dass keine lokalen kommissuralen Interneurone mit Eigenschaften einer bilateral die Extremitäten koordinierenden Funktion erfolgreich dargestellt werden konnten, können dazu keine weiteren Aussagen gemacht werden.

Ein anderer denkbarer Typ von bilateral die Extremitäten koordinierenden lokalen Interneuronen wäre einer, der in einem Hemiganglion sensorische Informationen von Mechanosensoren wie zum Beispiel von campaniformen Sensillen, Gelenkstellungsrezeptoren, spannungssensitiven Rezeptoren und Chordotonalorganen bezieht (HUSTERT 1978; BRÄUNIG and HUSTERT 1980; HUSTERT et al. 1981; BRÄUNIG et al. 1981; BURROWS 1982a,b, 1987b) und im anderen Hemiganglion auf Motound andere Interneurone verschaltet. Letztgenannte Mechanosensoren und Propriozeptoren projizieren größtenteils in die lateralen, dorsal gelegenen Neuropile (PFLÜGER et al. 1988), und in diesen Neuropilen verzweigen auch Moto- und Interneurone von lokalen neuronalen Netzwerken (TYRER and ALTMAN 1974; ALTMAN and TYRER 1977; ALTMAN 1980; WATSON 1984). Daher wäre für einen auf die oben genannte Weise kommunizierenden Typ von bilateral koordinierenden kommissuralen Interneuronen $\mathrm{zu}$ erwarten, dass seine bilateralen Verzweigungsbereiche in beiden Hemiganglien dorsal im Bereich der lateralen Neuropile (aLAC, pLAC) lokalisiert sind und dass diese Interneurone im Sinne einer schnellen Informationsübertragung zwischen den lokalen neuronalen Netzwerken in beiden Hemiganglien auch durch die dorsalen Kommissuren verlaufen. Im Rahmen der vorliegenden Arbeit wurde ein solcher Typ von lokalen kommissuralen Interneuronen mit einer potentiellen bilateral die Extremitäten koordinierenden Funktion jedoch nicht vorgefunden. Alle hier beschriebenen kommissuralen Interneurone mit einer möglichen Funktion im Kontext der Koordination der Extremitäten sind große intersegmentale Interneurone, deren Axone kontralateral ins Pro- und/oder Metathorakalganglion projizieren und bei welchen demnach zumindest keine ausschließlich bilateral koordinierende Funktion zu erwarten ist. 
4.5 Vergleich der kommissuralen Interneurone vom Typ CIN-I und CIN-II mit verschiedenen Klassen kommissuraler Interneurone, die für andere Tiergruppen beschrieben wurden

Bei allen hier vorgestellten kommissuralen Interneuronen (unabhängig von ihrem Verzweigungstyp) handelt es sich um große intersegmentale Interneurone. Sie haben in der Regel ein kontralateral zum Soma gelegenes Axon (Ausnahme: CINII(a)i1, Abb. 29 und CIN-II(a)i2, Abb. 35, deren jeweiliges Soma ipsilateral zum Axon liegt), das im Konnektiv auf- oder absteigt oder dort eine Bifurkation besitzt (im kontralateralen Konnektiv sowohl auf- als auch absteigend). Es wurde kein kommissurales Interneuron gefunden, dessen Axon in mehr als zwei intersegmentale Richtungen projiziert. Auch eine Crossover-Anordnung zwischen auf- und absteigenden Teilen des Axons (zum Beispiel im ipsilateralen Konnektiv aufsteigend und im kontralateralen Konnektiv absteigend oder umgekehrt) wurde bei keinem der Neurone festgestellt. Diese Befunde stimmen ganz gut mit der Morphologie mehrerer Klassen kommissuraler Interneurone mit anscheinend bilateral koordinierender Funktion überein, die für Wirbeltiere beschrieben wurden (Neunauge: BUCHANAN and COHEN 1982; Nagetiere: EIDE et al. 1999; STOKKE et al. 2002; NISSEN et al. 2005; Katze: MATSUYAMA et al. 2006). Dabei werden solche kommissuralen Interneurone unterschieden, deren Axone kontralateral im Rückenmark absteigen (dCINs: descending commissural interneurons), kontralateral aufsteigen (aCINs: ascending commissural interneurons), kontralateral durchlaufend sind (adCINs: ascending-descending commissural interneurons) und solche, die zwar intersegmental sein können, die aber nur über kurze Strecken (weniger als 1,5 Segmente) projizieren (sCINs: short range commissural interneurons). Die ersten drei genannten Klassen werden auch unter dem Sammelbegriff ICINs (long range commissural interneurons) zusammengefasst.

Die in der vorliegenden Arbeit beschriebenen kommissuralen Interneurone entsprechen morphologisch weitgehend den bereits beschriebenen „long range CINs“, zumindest hinsichtlich der Lage ihrer Axone (kontralateral zum Soma lokalisiert) und bezüglich der intersegmentalen Projektionsbereiche (aufsteigende, absteigende und durchlaufende Axone). Bei den für Wirbeltiere beschriebenen kommissuralen Interneuronen wird vor allem den absteigenden dCINs eine wichtige 
Rolle bei der bilateralen Koordination (von Extremitäten oder kontralateral gelegenen Muskelpartien der Körperflanken) zugeschrieben, und sie scheinen intrasegmental eine reziprok gerichtete Inhibition zwischen lokalen neuronalen Netzwerken für Lokomotion zu vermitteln. Es gibt Hinweise, dass auch die hier beschriebenen kommissuralen Interneurone, vor allem die vom Typ CIN-I, möglicherweise inhibitorisch verschalten (siehe Erläuterungen dazu unter Abschnitt 4.2).

Den sCINs (short range commissural interneurons) bei Wirbeltieren wird eher eine nebenläufige Rolle bei der bilateralen Koordination zugeschrieben (QUINLAN and KIEHN 2007), und sie sollen auch nur 10-15\% der Gesamtpopulation kommissuraler Interneurone ausmachen (STOKKE et al. 2002). Es wurde bereits gezeigt, dass sCINs alleine für eine bilaterale rechts-links-Koordination zumindest während Laufbewegungen nicht ausreichend sind (QUINLAN and KIEHN 2007). Ein mögliches Äquivalent $\mathrm{zu}$ den sCINs bei Wirbeltieren könnten bei Wanderheuschrecken lokale kommissurale Interneurone mit potentiell bilateral die Extremitäten koordinierender Funktion sein. Solche Neurone konnten jedoch nicht erfolgreich dargestellt werden (siehe Erläuterungen dazu unter Abschnitt 4.4). Möglicherweise kommen solche lokalen kommissuralen Interneurone mit potentiell bilateral die Extremitäten koordinierender Funktion auch im ZNS von Wanderheuschrecken nicht so zahlreich vor (siehe Abschnitt 4.4). Und vielleicht spielen solche lokalen kommissuralen Interneurone auch bei Insekten nur eine eher untergeordnete Funktion bei der bilateralen Koordination der Extremitäten.

Von den dCINs, aCINs und sCINs ist bekannt, dass sie direkt und indirekt über zwischengeschaltete Interneurone inhibitorisch oder erregend auf Motoneurone verschalten können, wobei vor allem dem inhibitorischen Verschaltungsweg eine wichtige Rolle bezüglich der Erzeugung der rhythmischen alternierenden motorischen Erregungsmuster zugeschrieben wird, wie sie bei der Lokomotion auftreten. Dies trifft möglicherweise auch auf die hier beschriebenen kommissuralen Interneurone vom Typ CIN-I zu, ist aber aufgrund der Datenlage nicht zu entscheiden. Die Rolle der adCINs (durchlaufendes Axon) bei der bilateralen Koordination ist zumindest im Falle von „fiktiven Laufmustern“ hingegen noch nicht genau bekannt, und es wird angenommen, dass sie vielleicht eher eine die Extremitäten koordinierende Funktion während nicht-rhythmischer Bewegungen 
spielen (ZHONG et al. 2006b). Auch viele der hier beschriebenen kommissuralen Interneurone haben ein im kontralateralen Konnektiv durchlaufendes Axon. Aber bei keinem der betreffenden Präparate traten rhythmische Muskelbewegungen oder Laufbewegungen auf, so dass darüber nichts Genaueres ausgesagt werden kann. 
Mesothorakale kommissurale Interneurone (CIN) im zentralen Nervensystem der Wanderheuschrecke Locusta migratoria migratorioides wurden durch intrazelluläre Ableitung mit der Glasmikroelektrode physiologisch auf eine mögliche bilateral die beiden Mittelbeine koordinierende Funktion untersucht und durch Färbung mit Lucifer Yellow morphologisch beschrieben. Sie haben Eigenschaften einer sowohl bilateral wie intersegmental die Extremitäten koordinierenden Funktion und wurden anhand ihres Verzweigungsmusters in zwei Gruppen (Typen) eingeteilt, die sich durch folgende Merkmale auszeichnen:

1. Kommissurale Interneurone vom Typ CIN-I haben bilaterale Verzweigungsbereiche in beiden Hemiganglien, die meistens dorsal oder intermediär bis dorsal lokalisiert sind und sich bis zu den dorsal-lateralen Neuropilregionen erstrecken können. Neben lokal auftretenden Kollateralen des Axons tragen oft auch manche Verzweigungen des primären Neuriten Varikositäten (potentieller lokaler Projektionsbereich des Neurons). Varikose Fasern können nebeneinander mit eher dendritischen vorkommen (scheinbar vermischte Input- und Outputregion), oder sie liegen in einem gesonderten Verzweigungsbereich (manchmal nur in einem Hemiganglion) vor. Alle gefundenen Neurone dieses Typs haben kontralateral zum Soma gelegene Axone. Wenn durch dorsal-taktile Tarsusreize an einem der Mittelbeine bilaterale und intersegmentale Reflexe und damit Bewegungen der Mittelbeine ausgelöst werden, erscheint neuronal erhöhte Aktivität von CIN-I Neuronen oft streng korreliert (synchron, aber auch alternierend) mit den parallel gemessenen Muskelaktivitäten, jedoch selten unabhängig davon. Manchmal werden solche Neurone bei Tarsusreizung auf einer Seite unidirektional synchron zu einer der auftretenden Muskelaktivitäten gehemmt.

2. Kommissurale Interneurone vom Typ CIN-II haben einen median gelegenen Hauptverzweigungsbereich. Sie können auch in tiefer gelegenen Regionen im Ganglion verzweigen, meistens jedoch verzweigen sie intermediär bis dorsal. Die Verzweigungen des primären Neuriten reichen nicht bis an die dorsal-lateralen Neuropile heran. Allerdings tragen nicht wenige Neurone dieses Typs lokale Kollaterale, die sich unilateral bis dorthin erstrecken können. Auch Verzweigungen vom primären Neuriten können Varikositäten tragen (potentielle Outputregionen), die dann in der Regel nebeneinander mit eher dendritischen (mutmaßliche Inputregionen) vorkommen. Die Axone können kontralateral oder ipsilateral zum Soma gelegen sein. Auch CIN-II Neurone zeigen häufig neuronale Erregung im Kontext von durch taktile Reizung ausgelösten Bewegungen eines oder beider Mittelbeine. Die Korrelation zu den gemessenen Muskelaktivitäten kann aber im Verlauf einer Aufzeichnung etwas variabler sein, neuronale Bursts können manchmal präaktiv zu einer der Muskelaktivitäten erscheinen, manchmal exakt synchron, aber gelegentlich auch leicht verzögert. Neurone dieses Typs werden bei taktiler Reizung an einem der Mittelbeine häufig unidirektional gehemmt in der Phase einer der auftretenden Muskelaktivitäten. 
Sowohl CIN-I wie CIN-II Neurone sind meistens große intersegmentale Interneurone mit Projektionsbereichen in benachbarten Ganglien. Beide Typen kommissuraler Interneurone sind in den dorsalen Kommissuren DCI-DCVI vorzufinden und haben ein ventral gelegenes Soma. Ihre Axone können aufsteigend oder absteigend sein, oder sie haben eine Bifurkation im kontralateralen Konnektiv. Das ist aus morphologischer Sicht weitgehend vergleichbar mit verschiedenen Gruppen von kommissuralen Interneuronen, die für Vertebraten (Neunaugen, Nagetiere) und auch für Invertebraten (Krebs, Wanderheuschrecken) beschrieben wurden. Den meisten dieser Klassen kommissuraler Interneurone wird eine Funktion im Kontext der bilateralen Koordination zugeschrieben (bei Vertebraten und beim Krebs). Von den hier beschriebenen Neuronen haben vor allem die bilateral verzweigenden intersegmentalen Interneurone vom Typ CIN-I Eigenschaften einer die Extremitäten sowohl bilateral wie intersegmental koordinierenden Funktion. $\mathrm{Da}$ sie im Mesothorakalganglion überwiegend bilaterale, dorsal gelegene Verzweigungsbereiche in der Region der lateralen Neuropile haben, stellt sich die Frage, ob die bilaterale Koordination der Extremitäten bei Insekten auf neuraler Ebene möglicherweise zum Teil auch über solche intersegmentalen kommissuralen Neurone erfolgt, welche

- kontralateral benachbarte Extremitäten bilateral und gleichzeitig ipsilateral benachbarte Extremitäten intersegmental koordinieren (Multifunktionalität),

- vor allem propriozeptive sensorische Informationen (ipsi- oder bilateral) über Gelenkstellungen und Bewegungen einzelner Extremitäten erhalten, vorwiegend durch dorsale Kommissuren verlaufen und (kontra- oder bilateral) direkt auf Moto- und Interneurone von lokalen neuronalen Netzwerken der Lokomotion verschalten.

Da die hier beschriebenen Neurone vom Typ CIN-II ebenfalls in den Kontext zumindest einer allgemeineren Koordination der Extremitäten involviert zu sein scheinen und oftmals im näheren Umfeld $\mathrm{zu}$ beiden Seiten der dorsalen Kommissuren verzweigen, wird ein weiterer Aspekt diskutiert. Dieser betrifft die Fragestellung, ob in einem median und intermediär bis dorsal im Ganglion gelegenen Bereich in der näheren Umgebung der dorsalen Kommissuren DCI-DCVI eine potentielle „neuronale Schnittstelle“ lokalisiert ist, wo verschiedene Interneurone, die an der intersegmentalen Koordination der Extremitäten beteiligt sind, auf andere lokale oder intersegmentale Interneurone verschalten, die in die bilaterale Koordination involviert sind. 
ALTMAN, J.S. (1980). Functional organisation of insect ganglia. In: Neurobiology of invertebrates. Ed. J. Salanski. Adv. Physiol. Sci. 23: 537-555.

ALTMAN J.S., TYRER, N.M. (1977). The locust wing hinge stretch receptor. I. Primary sensory neurons with enormous central arborizations. J. Comp. Neurol. 172: 409-430.

BÄSSLER, U. (1983). Neural basis of elementary behavior in stick insects. New York: Springer-Verlag.

BÄSSLER, U. (1986). On the definition of central pattern generator and its sensory control. Biol. Cybern. 54: 65-69.

BÄSSLER, U. (1993). The walking- (and searching-) pattern generator of stick insects, a modular system composed of reflex chains and endogenous oscillators. Biol. Cybern. 69: 305-317.

BENTLEY, D.R. (1970). A topological map of the locust flight system motor neurons. J. Insect. Physiol. 16: 905-918.

BERKOWITZ, A., LAURENT, G. (1996). Central generation of grooming motor patterns and interlimb coordination in locusts. J. Neurosci. 16: 8079-8091.

BIRINYI, A., VISZOKAY, K., WEBER, I., KIEHN, O., ANTAL, M. (2003). Synaptic targets of commissural interneurons in the lumbar spinal cord of neonatal rats. J. Comp. Neurol. 461: 429-440.

BIRÓ, Z., Hill, R.H., Grillner, S. (2006). 5-HT modulation of identified segmental premotor interneurons in the lamprey spinal cord. J. Neurophysiol. 96: 931935.

BIRÓ, Z., Hill, R.H., Grillner, S. (2008). The Activity of Spinal Commissural Interneurons During Fictive Locomotion in the Lamprey. J. Neurophysiol. 100: 716-722.

BLICKHAN, R., FULL, R. J. (1987). Locomotion energetics of the ghost crab. II. Mechanics of the centre of mass during walking and running. J. Exp. Biol. 130: 155-174.

BORGMANN, A., SCHARSTEIN, H., BÜSCHGES, A. (2007). Intersegmental Coordination: Influence of a single walking leg on the neighboring segments in the stick insect walking system. J. Neurophysiol. 98: 1685-1696.

BRANCHEREAU, P., MORIN, D., BONNOT, A., BALLION, B., CHAPRON, J., VIALA, D. (2000). Development of lumbar rhythmic networks: from embryonic to neonate locomotor-like patterns in the mouse. Brain Res. Bull. 53: 711-718. 
BRÄUNIG, P., HUSTERT, R. (1980). Proprioceptors with central cell bodies in insects. Nature, Lond. 283: 768-770.

BRÄUNIG, P. (1982a). The peripheral and central nervous organization of the locust coxo-trochanteral joint. J. Neurobiol. 13: 413-433.

BRÄUNIG, P. (1982b). Strand receptors with central cell bodies in the proximal leg joints of orthopterous insects. Cell Tissue Res. 222: 647-654.

BRÄUNIG, P., HUSTERT, R. (1985). Actions and interactions of proprioceptors of the locust hind leg coxo-trochanteral joint. I. Afferent responses in relation to joint position and movement. J. Comp. Physiol. 157A: 73-82.

BRÄUNIG, P., HUSTERT, R. (1985). Actions and interactions of proprioceptors of the locust hind leg coxo-trochanteral joint. II. Influence on the motor system. J. Comp. Physiol. 157A: 83-89.

BRÄUNIG, P., HUSTERT, R., PFLÜGER, H.J. (1981). Distribution and specific central projections of mechanoreceptors in the thorax and proximal leg joints of locusts. I. Morphology, location and innervation of internal proprioceptors of pro- and metathorax and their central projections. Cell Tissue Res. 216: 51-11.

BUCHANAN, J.T. (1999). Commissural interneurons in rhythm generation and intersegmental coupling in the lamprey spinal cord. J. Neurophysiol. 81: 2037-2045.

BUCHANAN, J.T. (1986). Premotor interneurons in the lamprey spinal cord: morphology, synaptic interactions, and activities during fictive swimming. In: Neurobiology of Vertebrate Locomotion, edited by S. Grillner, P.S.G. Stein, D.G. Stuart, H. Forssberg, R.M. Herman. London: MacMillan, p. 321-334.

BUCHANAN, J.T., COHEN, A.H. (1982). Activities of identified interneurons, motoneurons, and muscle fibers in the lamprey and effects of reticulospinal and dorsal cell stimulation. J. Neurophysiol. 47: 948-960.

BUCHANAN, J.T., GRILLNER, S. (1987). Newly identified "glutamate interneurons" and their role in locomotion in the lamprey spinal cord. Science 236: 312314. 1987.

BURNS, M.D. (1973). The control of walking in Orthoptera. I. Leg movements in normal walking. J. Exp. Biol. 58: 45-58.

BURROWS, M. (1979b). Graded synaptic transmission between local premotor interneurons of the locust. J. Neurophysiol. 42: 1108-1123.

BURROWS, M. (1980a). The control of sets of motoneurones by local interneurones in the locust. J. Physiol. London 298: 213-233. 
BURROWS, M. (1985). The processing of mechanosensory information by spiking local interneurones in the locust. J. Neurophysiol. 54: 463-478.

BURROWS, M. (1987a). Inhibitory interactions between spiking and nonspiking local interneurones in the locust. J. Neurosci. 7: 3282-3292.

BURROWS, M. (1987b). Parallel processing of proprioceptive signals by spiking local interneurons and motorneurons in the locust. J. Neurosci. 7: 1064-1080.

BURROWS, M. (1988). Responses of spiking local interneurones in the locust to proprioceptive signals from the femoral chordotonal organ. J. Comp. Physiol. 164A: 207-217.

BURROWS, M., HOYLE, G. (1973). The mechanism of rapid running in the ghost crab, Ocypode ceratophthalma. J. Exp. Biol. 58: 327-349.

BURROWS, M., PFLÜGER, H.J. (1988). Positive feedback loops from proprioceptors involved in leg movements of the locust. J. Comp. Physiol. 163A: 425-440.

BURROWS, M., SIEGLER, M.V.S. (1982). Spiking local interneurons mediate local reflexes. Science 217: 650-652.

BURROWS, M., SIEGLER, M.V.S. (1984). The morphological diversity and receptive fields of spiking local interneurons in the locust metathoracic ganglion. J. Comp. Neurol. 224: 483-508.

BURROWS, M. (1996). The neurobiology of an insect brain. Oxford University Press Inc., New York.

BUTT, S.J., KIEHN, O. (2003). Functional identification of interneurons responsible for left-right coordination of hindlimbs in mammals. Neuron 38: 953-963.

BUTT S.J., HARRIS-WARRICK, R.M., KIEHN, O. (2002). Firing properties of identified interneuron populations in the mammalian hindlimb central pattern generator. J. Neurosci. 15: 9961-9971.

CARLIN, K.P., DAI, Y., JORDAN, L.M. (2006). Cholinergic and serotonergic excitation of ascending commissural neurons in the thoraco-lumbar spinal cord of the neonatal mouse. J. Neurophyiol. 95: 1278-1284.

CAVAGNA, G.A., HEGLUND, N.C., TAYLOR, C.R. (1977). Mechanical work in terrestrial locomotion: Two basic mechanisms for minimizing energy expenditure. Am. J. Physiol. 233: 243-261.

CAZALETS, J.R., SQUALLI-HOUSSAINI, Y., CLARAC, F. (1992). Activation of the central pattern generators for locomotion by serotonin and excitatory amino acids in neonatal rat. J. Physiol. (Lond.) 455: 187-204. 
CAZALETS J.R., BORDE, M., CLARAC, F. (1995). Localization and organization of the central pattern generator for hindlimb locomotion in newborn rat. J. Neurosci. 15: 4943-4951.

CLEMENTS, A.N., MAY, T.E. (1974).Studies on locust neuromuscular physiology in relation to glutamic acid. J. Exp. Biol. 60: 673-705.

COHEN, A.H., WALLÉN, P. (1980). The neuronal correlate of locomotion in fish. "Fictive swimming" induced in an in vitro preparation of the lamprey spinal cord. Exp. Brain Res. 41: 11-18.

COHEN, A.H., ERMENTROUT, G.B., KIEMEL, T., KOPELL, N., SIGVARDT, K.A., WILLIAMS, T.L. (1992). Modeling of intersegmental coordination in the lamprey central pattern generator for locomotion. Trends Neurosci. 15: 434438.

CRUSE, H. (1990). What mechanisms coordinate leg movements in walking arthropods? Trends Neurosci. 13: 15-21.

DELCOMYN, F. (1971a). The locomotion of the cockroach Periplaneta americana. J. Exp. Biol. 54: 443-452.

DELCOMYN, F. (1971b). The effects of limb amputation on locomotion in the cockroach Periplaneta americana. J. Exp. Biol. 54: 453-469.

DELCOMYN, F. (1980). Neural basis of rhythmic behavior in animals. Science 210: 492-498.

DÜRR, V. (2005). Context-dependent changes in strength and efficiacy of leg coordination mechanisms. J. Exp. Biol. 208: 2253-2267.

EDGLEY, S.A., JANKOWSKA, E., HAMMAR, I. (2004). Ipsilateral actions of feline corticospinal tract neurons on limb motoneurons. J. Neurosci. 24: 78047813.

EIDE, A.L., GLOVER, J., KJAERULFF, O., KIEHN, O. (1999). Characterization of commissural interneurons in the lumbar region of the neonatal rat spinal cord. J. Comp. Neurol. 403: 332-345.

EPSTEIN, S., GRAHAM, D. (1983). Behavior and motor output for an insect walking on a slippery surface. 1. Forward walking. J. Exp. Biol. 105: 215-229.

FOTH, E., BÄSSLER, U. (1985a). Leg movements of stick insects walking with five legs on a treadwheel and with one leg on a motor-driven belt. I. General results and 1:1-coordination. Biol. Cybern. 51: 313-318.

FOTH, E., BÄSSLER, U. (1985b). Leg movements of stick insects walking with five legs on a treadwheel and with one leg on a motor-driven belt. II. Leg coordination when step-frequencies differ from leg to leg. Biol. Cybern. 51: 319-324. 
FRIESEN, W.O., HOCKER, C.G. (2001). Functional analyses of the leech swim oscillator. J. Neurophysiol. 86: 824-835.

FRIESEN, W.O., PEARCE, R.A. (1993). Mechanisms of intersegmental coordination in leech locomotion. Semin. Neurosci. 5: 41-47.

FULL, R. J., TU, M. S. (1990). The mechanics of six-legged runners. J. Exp. Biol. 148: $129-146$.

GOODMAN, C.S. (1959). Hair plates on the first cervical sclerites of the Orthoptera. Nature (Lond.) 183: 1106-1107.

GRAHAM, D. (1972). A behavioural analyses of the temporal organization of walking movements in the first instar and adult stick insect Carausius morosus. J. Comp. Physiol. 81A: 23-52.

GRAHAM, D. (1979). Effects of circum-oesophageal lesion on the behaviour of the stick insect Carausius morosus. I. Cyclic behaviour patterns. Biol. Cybern. 32: 139-145.

GRAHAM, D. (1985). Influence of coxa-thorax joint receptors on retractor motor output during walking in Carausius morosus. J. Exp. Biol. 114: 131-139.

GRAHAM, D., CRUSE, H. (1981). Coordinated walking of stick insects on a mercury surface. J. Exp. Biol. 92: 229-241.

GREGORY, G.E. (1974). Neuroanatomy of the mesothoracic ganglion of the cockroach Periplaneta americana (L.). I. The roots of the peripheral nerves. Phil. Trans. R. Soc. Lond. 267B: 421-465.

GREGORY, G.E. (1985). Neuroanatomy of the mesothoracic ganglion of the cockroach Periplaneta americana (L.). II. Median neuron cell body groups. Phil. Trans. R. Soc. Lond. 306B: 191-218.

HAMMAR, I., BANNATYNE, B.A., MAXWELL, D.J., EDGLEY, S.A., JANKOWSKA, E. (2004). The actions of monoamines and distribution of noradrenergic and serotoninergic contacts on different subpopulations of commissural interneurons in the cat spinal cord. Eur J Neurosci 19:1305-1316.

HEGLUND, N.C, CAVAGNA, G.A., TAYLOR, C.R. (1982a). Energetics and mechanics of terrestrial locomotion. III. Energy changes of the centre of mass as a function of speed and body size in birds and mammals. /. exp. Biol. 79, 41-56.

HEGLUND, N. C , FEDAK, M. A., TAYLOR, C. R. AND CAVAGNA, G. A. (1982b). Energetics and mechanics of terrestrial locomotion. IV. Total mechanical energy changes as a function of speed and body size in birds and mammals. J. Exp. Biol. 97: 57-66. 
HEINRICH, R., ELSNER, N. (1997). Central nervous control of hindleg coordination in stridulating grasshoppers. J. Comp. Physiol. 180A: 257-269.

HEITLER, W. (1980). Neural mechanisms of central pattern generation in the crayfish swimmeret system. Adv. Physiol. Sci. 23: 369-383.

HELLGREN-KOTALESKI, J., LANSNER, A., GRILLNER, S. (1999). Neural mechanisms potentially contributing to the intersegmental phase lag in lamprey. II. Hemisegmental oscillations produced by mutually coupled excitatory neurons. Biol. Cybern. 81: 299-315.

HILL, A.A.V., MASINO, M.A., CALABRESE, R.L. (2003). Intersegmental coordination of rhythmic motor patterns. J. Neurophysiol. 90: 531-538.

HOCKER, C.G., YU, X., FRIESEN, W.O. (2000). Functionally heterogeneous segmental oscillators generate swimming in the medical leech. J. Comp. Physiol. 186A: 871-883.

HÖLTJE M., HUSTERT R. (2003). Rapid mechano-sensory pathways code leg impact and elicit very rapid reflexes in insects. J. Exp. Biol. 206: 2715-2724.

HOOVER, J.E., DURKOVIC, R.G. (1992). Retrograde labeling of lumbosacral interneurons following injections of red and green fluorescent microspheres into hindlimb motor nuclei of the cat. Somatosens. Mot. Res. 9: 211-226.

HUGHES, G.M., WIERSMA, C.A.G. (1960). The co-ordination of swimmeret movements in the crayfish, Procambarus clarkii. J. Exp. Biol. 37: 657-670.

HUSTERT, R. (1978). Segmental and interganglionic projections from primary fibres of insect mechanoreceptors. Cell. Tissue Res. 194: 337-351.

HUSTERT, R. (1985). Multisegmental integration and divergence of afferent information from single tactile hairs in a cricket. J. Exp. Biol. 118: 209-227.

HUSTERT, R. (1989). Mesothoracic interneurons may coordinate the legs of locusts. In: Neural Mechanisms of Behavior. Proc. Of the $2^{\text {nd }}$ Int. Congress of Neuroethology. September 10-16.

HUSTERT, R., PFLÜGER, H.J., BRÄUNIG, P. (1981). Distribution and specific central projections of mechanoreceptors in the thorax and proximal leg joints of locusts. III. The external mechanoreceptors: The campaniform sensilla. Cell Tissue Res. 216: 97-111.

JANKOWSKA, E., HAMMAR, I., SLAWINSKA, U., MALESZAK, K., EDGLEY, S.A. (2003). Neuronal basis of crossed actions from the reticular formation on feline hindlimb motoneurons. J. Neurosci. 23: 1867-1878.

JANKOWSKA, E., EDGLEY, S.A., KRUTKI, P., HAMMAR, I. (2005). Functional differentiation and organization of feline midlumbar commissural interneurons. J. Physiol. (Lond.) 565: 645-658. 
KIEHN, O., KJAERULFF, O. (1998). Distribution of central pattern generators for rhythmic motor outputs in the spinal cord of limbed vertebrates. Ann. NY Acad. Sci. 860: 110-129.

KIEN, J. (1980). Morphology of locust neck muscle motoneurons and some of their inputs. J. Comp. Physiol. 140: 321-336.

KJAERULFF, O., KIEHN, O. (1996). Distribution of networks generating and coordinating locomotor activity in the neonatal rat spinal cord: a lesion study. J. Neurosci. 16: 5777-5794.

KRUTKI, P., JANKOWSKA, E., EDGLEY, S.A. (2003). Are crossed actions of reticulospinal and vestibulospinal neurons on feline motoneurons mediated by the same or separate commissural neurons? J. Neurosci. 23: 80418050 .

LANUZA, G.M., GOSGNACH, S., PIERANI, A., JESSELL, T.M., GOULDING, M. (2004). Genetic identification of spinal interneurons that coordinate left-right locomotor activity necessary for walking movements. Neuron 42: 375-386.

LAURENT, G.J. (1987) The morphology of a population of thoracic intersegmental intemeurons in the locust. J. Comp. Neurol. 256: 412-429.

LAURENT, G.J., BURROWS, M. (1988). A population of ascending intersegmental interneurones in the locust with mechanosensory inputs from a hind leg. J. Comp. Neurol. 275: 1-12.

LAURENT, G.J., BURROWS, M. (1989a). Distribution of intersegmental inputs to nonspiking local interneurons and motor neurons in the locust. J. Neurosci. 9: 3019-3029.

LAURENT, G.J., BURROWS, M. (1989b). Intersegmental interneurons can control the gain of reflexes in adjacent segments of the locust by their action on nonspiking local interneurons. J. Neurosci. 9: 3030-3039.

LAURENT, G.J., HUSTERT, R. (1988). Motorneuronal receptive fields delimit patterns of activity during locomotion of the locust. J. Neurosci. 8: 43494366.

MATSUYAMA, K., KOBAYASHI, S., AOKI, M. (2006). Projection patterns of lamina VIII commissural neurons in the lumbar spinal cord of the adult cat: an anterograde neural tracing study. Neuroscience 140: 203-218.

MULLONEY, B., HALL, W. (2003). Local commissural interneurons integrate information from intersegmental coordinating interneurons. J. Comp. Neurol. 466: 366-376.

MURCHISON, D., CHRACHRI, A., MULLONEY, B. (1993). A separate local patterngenerating circuit controls the movements of each swimmeret in crayfish. J. Neurophysiol. 70: 2620-2631. 
MURPHEY, R.K., BACON, J.P., SAKAGUCHI, D.S., JOHNSON, S.E. (1983). Transplantation of cricket sensory neurons to ectopic locations: arborizations and synaptic connections. J. Neurosci. 3: 659-672.

MURPHEY, R.K., BACON, J.P., JOHNSON, S.E. (1985). Ectopic neurons and the organization of insect sensory systems. J. Comp. Physiol. 156A: 381-389.

NAGAYAMA, T., BURROWS, M. (1990). Input and output connections of an anteromedial group of spiking local interneurons in the metathoracic ganglion of the locust. J. Neurosci. 10: 785-794.

NEWLAND, P.L., BURROWS, M. (1997). Processing of tactile information in neuronal networks controlling leg movements of the locust. J. Insect Physiol. 43: 107-123.

NISSEN, U.V., MOCHIDA, H., GLOVER, J.C. (2005). Development of projectionspecific interneurons and projection neurons in the embryonic mouse and rat spinal cord. J. Comp. Neurol. 483: 30-47.

PEARSON, K.G., FOURTNER, C.R. (1975). Non-spiking interneurons in the walking system of the cockroach. J. Neurophysiol. 38: 33-52.

PEARSON, K.G. (1985). Are there central pattern generators for walking and flight in insects? In: Feedback and motor control in invertebrates and vertebrates (Barnes WJP, ed), pp 307-315. London: Croom Helm.

PFLÜGER, H.J. (1980). Central nervous projections of sternal trichoid sensilla in locusts. Naturwissenschaften 67: 316.

PFÜGER, H.J. (1984). The large fourth abdominal intersegmental interneuron: a new type of wind-sensitive ventral cord interneuron in locusts. J. Comp. Neurol. 222, 343-357.

PFLÜGER, H.J., BRÄUNIG, P., HUSTERT, R. (1981). Distribution and specific central projections of mechanoreceptors in the thorax and proximal leg joints of locusts. II. The external mechanoreceptors: hair plates and tactile hairs. Cell Tissue Res. 216: 79-96.

PFLÜGER, H.J., BRÄUNIG, P., HUSTERT, R. (1988). The organization of mechanosensory neuropiles in the locust thoracic ganglia. Phil. Trans. R. Soc. Lond. 321B: 1-26.

PIPA, R.L., COOK, E.F., RICHARDS, A.G. (1959). Studies on the hexapod nervous system. II. The histology of the thoracic ganglia of the adult cockroach, Periplaneta americana (L.). J. Comp. Neurol. 113: 401-433.

QUINLAN, K.A., KIEHN, O. (2007). Segmental, synaptic actions of commissural interneurons in the mouse spinal cord. J. Neurosci. 27(24): 6521-6530. 
RAMIREZ, J.M., PEARSON, K.G. (1988). Generation of motor patterns for walking and flight in motoneurons supplying bifunctional muscles in the locust. J. Neurobiol., 19: 257-282.

ROBERTS, A., SOFFE, S.R., WOLF, E.S., YOSHIDA, M., ZHAO, F.Y. (1998). Central circuits controlling locomotion in young frog tadpoles. Ann. NY Acad. Sci. 860: 19-34.

ROEDER, K.D. (1937). The control of tonus and locomotor activity in the praying mantis (Mantis religiosa L.). J. Exp. Zool. 76: 353-374.

ROEDER, K.D. (1948). Organization of the ascending giant fiber system in the cockroach (Periplaneta americana). J. Exp. Zool. 108: 243-261.

RYCKEBUSCH, S., LAURENT, G.L. (1993). Rhythmic patterns evoked in locust leg motor neurons by the muscarinic agonist pilocarpine. J. Neurophysiol. 69: 1583-1595.

SCHMITZ, J., HAßFELD, G. (1989). The treading-on-tarsus reflex in stick insects: phase-dependence and modifications of the motor output during walking. J. Exp. Biol. 143: 373-388.

SIEGLER, M.V.S., BURROWS, M. (1979). The morphology of local nonspiking interneurons in the metathoracic ganglion of the locust. J. Comp. Neurol. 183: $121-148$.

SIEGLER, M.V.S., BURROWS, M. (1984). The morphology of two groups of spiking local interneurones in the metathoracic ganglion of the locust. J. Comp. Neurol. 244: 463-482.

SKINNER, F.K., MULLONEY, B. (1998a). Intersegmental coordination in invertebrates and vertebrates. Curr. Opin. Neurobiol. 8: 725-732.

SKORUPSKI, P., HUSTERT, R. (1991). Short communication reflex pathways responsive to depression of the locust coxotrochanteral joint. J. Exp. Biol. 158: 599-605.

SNODGRASS R. E. (1929). The thoracic mechanism of a grashopper and its antecedents. Smithson. Misc. Coll. 82: No. 2.

SOFFE, S.R., CLARKE, J.D., ROBERTS, A. (1984). Activity of commissural interneurons in spinal cord of Xenopus embryos. J. Neurophysiol. 51: 12571267.

STOKKE, M.F., NISSEN, U.V., GLOVER, J.C., KIEHN, O. (2002). Projection patterns of commissural interneurons in the lumbar spinal cord of the neonatal rat. J. Comp. Neurol. 446: 349-359.

TYRER, N.M., ALTMAN, J.S. (1974). Motor and sensory flight neurones in a locust demonstrated using cobalt chloride. J. Comp. Neurol. 157: 117-138. 
TYRER, N.M., GREGORY, G.E. (1982). A guide to the neuroanatomy of locust suboesophageal and thoracic ganglia. Phil. Trans. R. Soc. Lond. 297B: 91123.

WALLÉN, P., WILLIAMS, T.L. (1984). Fictive locomotion in the lamprey spinal cord in vitro compared with swimming in the intact and spinal animal. J. Physiol. (Lond.) 347: 225-239.

WATSON, A.H.D. (1984). The dorsal unpaired median neurons of the locust metathoracic ganglion: Neuronal structure and diversity, and synapse distribution. J. Neurocytol. 13: 303-327.

WATSON, A.H.D., BURROWS, M. (1983). The morphology, ultrastructure, and distribution of synapses on an intersegmental interneurone of the locust. J. comp. Neurol. 214: 154-169.

WATSON, A.H.D., BURROWS, M. (1987). Immunocytochemical and pharmacological evidence for GABAergic spiking local interneurones in the locust. J. Neurosci. 7: 1741-1751.

WENDLER, G. (1964). Laufen und Stehen der Stabheuschrecke Carausius morosus: Sinnesborstenfelder in den Beingelenken als Glieder von Regelkreisen. Z. vergl. Physiol. 48: 198-250.

WILSON, J.A. (1979). The structure and function of serially homologous leg motoneurones in the locust. I. Anatomy. J. Neurobiol. 10: 41-65.

WILSON, J.A. (1981). Unique, identifiable nonspiking interneurons in the locust mesothoracic ganglion. J. Neurobiol. 12: 353-366.

WILSON, J.A., PHILLIPS, C.E. (1982). Locust local nonspiking interneurons which tonically drive antagonistic motor neurons: physiology, morphology and ultrastructure. J. Comp. Neurol. 204: 21-31.

ZHONG, G., DÍAZ-RÍOZ, M., HARRIS-WARRICK, R.M. (2006a). Serotonin modulates the properties of ascending commissural interneurons in the neonatal mouse spinal cord. J. Neurophysiol. 95: 1545-1555.

ZHONG, G., DÍAZ-RÍOZ, M., HARRIS-WARRICK, R.M. (2006b). Intrinsic and functional differences among commissural interneurons during fictive locomotion and serotonergic modulation in the neonatal mouse. $\mathrm{J}$. Neurosci. 26(24): 6509-6517. 
Mein besonderer Dank gilt meinen Eltern und meiner Familie, die mich in meinem bisherigen Leben immer auf jede nur erdenkliche Weise so unterstützt haben, wie man es sich nur wünschen kann. Ohne euch wäre dies alles nicht möglich gewesen.

Auch Herrn Professor Dr. Reinhold Hustert bin ich zu großem Dank für die intensive und optimale Betreuung meines Dissertationsthemas verpflichtet. Neben materieller Unterstützung hat er mir mit seiner umfangreichen Erfahrung viele Tipps und nützliche Denkanstöße in kniffligen Fällen gegeben. Darüber hinaus sorgt seine ruhige, besonnene und freundliche Art stets für ein sehr angenehmes Arbeitsklima in unserer Abteilung.

Meine Schwester Verena Baldus verdient meine besondere Anerkennung und großes Lob insbesondere auch für das geduldige Korrekturlesen der Arbeit.

Sehr dankbar bin ich auch meiner Freundin Verena Merz, die mich die ganze Zeit über unterstützt und an mich geglaubt hat, und die immer für mich da ist.

Allen Mitarbeitern unserer Abteilung, sowie Rebecca Klug danke ich für ihre Unterstützung, Ratschläge und für eine ausgesprochen angenehme Arbeitsatmosphäre. Dies gilt im Besonderen auch für unsere biologisch-technische Assistentin Silvia Gubert, die mir bei der Anfertigung der Schnittserien und deren Analyse unter dem Elektronenmikroskop sehr hilfreich zur Seite gestanden hat.

Herrn Dr. Heribert Gras und Peter Gumrich danke ich für kritische Anmerkungen und nützliche Tipps bezüglich der Datenauswertung mit MS Excel. 


\section{CURRICULUM VITAE}

\section{Studium und wissenschaftlicher Bildungsgang}

2004

$03 / 2003-11 / 2003$

$11 / 2002$

2001 - heute

$1999-2003$

04/1999

$10 / 1996-11 / 2003$
Zulassung als Doktorand an der Georg-August-Universität Göttingen und Beginn der Dissertation am Johann-FriedrichBlumenbach-Institut für Zoologie \& Anthropologie, in der Arbeitsgruppe Sensomotorik; Betreuung der Arbeit durch Herrn Prof. Dr. Reinhold Hustert

Anfertigung der Diplomarbeit „Zeitverlauf und motorische Grundlagen des Landungssystems der Wüstenheuschrecke Schistocerca gregaria“ im Institut für Zoologie und Anthropologie der Universität Göttingen; Betreuung der Arbeit durch Herrn Prof. Dr. Reinhold Hustert

Erlangung des Diplom-Grades in Biologie

Diplomprüfung

Fächerkombination: Zoologie, Botanik, Psychologie

Tätigkeit als studentische/wissenschaftliche Hilfskraft im Johann-Friedrich-Blumenbach-Institut für Zoologie \& Anthropologie der Universität Göttingen, Arbeitsgruppe Sensomotorik, bei Herrn Prof. Dr. Reinhold Hustert

Hauptstudium der Biologie an der Georg-August-Universität Göttingen

Vordiplomprüfung

Studium der Biologie (Diplom-Studiengang) an der GeorgAugust-Universität Göttingen, Biologische Fakultät

\section{Schulbildung / Wehrdienst}

